\title{
Intertemporal Choice AND \\ Competitive Equilibrium
}

Kirsten I.M. Rohde 
(C) Kirsten I.M. Rohde, 2006

Published by Universitaire Pers Maastricht ISBN-10 9052785503

ISBN-13 9789052785509

Printed in The Netherlands by Datawyse Maastricht 


\title{
InTERTEMPORAL CHOICE AND \\ Competitive Equilibrium
}

\author{
PROEFSCHRIFT
}

ter verkrijging van de graad van doctor aan de Universiteit Maastricht,

op gezag van de Rector Magnificus, prof. mr. G.P.M.F. Mols

volgens het besluit van het College van Decanen,

in het openbaar te verdedigen op

vrijdag 13 oktober 2006 om 14.00 uur

door

Kirsten Ingeborg Maria Rohde

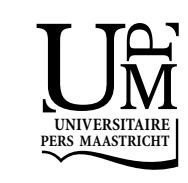




\section{Promotores:}

Prof. dr. P.J.J. Herings

Prof. dr. P.P. Wakker

\section{Beoordelingscommissie:}

Prof. dr. H.J.M. Peters (voorzitter)

Prof. dr. T. Hens (University of Zürich, Switzerland)

Prof. dr. A.M. Riedl 


\section{Contents}

Acknowledgments $\quad$ v

1 Introduction 1

1.1 Intertemporal Choice . . . . . . . . . . . . . . . . 2

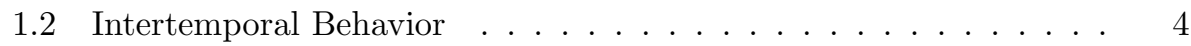

1.3 General Equilibrium . . . . . . . . . . . . . . . 5

I Intertemporal Choice $\quad 9$

2 Koopmans' Constant Discounting: A Simplification and an Extension to Incorporate Economic Growth 11

2.1 Introduction . . . . . . . . . . . . . . . . . . . . 11

2.2 The Result . . . . . . . . . . . . . . . . . . 13

2.3 Related Literature . . . . . . . . . . . . . . . . . . . 18

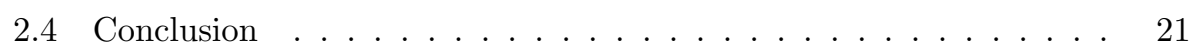

2.5 Appendix A. Proofs . . . . . . . . . . . . . . . . . . 21

2.6 Appendix B. Example . . . . . . . . . . . . . . . 25

3 The Hyperbolic Factor: a Measure of Decreasing Impatience $\quad 27$

3.1 Introduction . . . . . . . . . . . . . . . . . . 27

3.2 The Hyperbolic Factor Defined . . . . . . . . . . . . 30

3.3 The Hyperbolic Factor and Discounted Utility . . . . . . . . . . 32

3.3 .1 Constant Discounting . . . . . . . . . . . . . . 33

3.3.2 Generalized Hyperbolic Discounting . . . . . . . . . . . 33

3.3.3 Harvey Discounting . . . . . . . . . . . . . . . . . . . 34 
3.3.4 Proportional Discounting . . . . . . . . . . . . . 34

3.3.5 Quasi-hyperbolic Discounting ............ . . 35

3.4 Conclusion ............................ 35

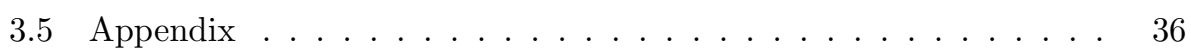

4 Time-Tradeoff Sequences for Quantifying and Visualising the Degree of Time Inconsistency, Using only Pencil and Paper 39

4.1 Introduction . . . . . . . . . . . . . . . . . . 39

4.2 Discounted Utility . . . . . . . . . . . . . . . . . . . . . . 42

4.3 Deriving the Degree of Time Inconsistency from TTO-Sequences . 44

4.4 TTO-Sequences to Measure Proneness to Arbitrage . . . . . . . . . 46

4.5 Illustrations of TTO-Sequences . . . . . . . . . . . . . . . . 49

4.6 Deriving the Discount Function from TTO-Sequences . . . . . . . 52

4.7 Method of Experiment . . . . . . . . . . . . . . 55

4.8 Results . . . . . . . . . . . . . . . . . 57

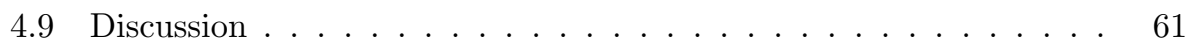

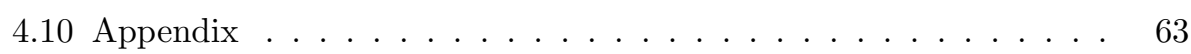

\section{General Equilibrium $\quad 67$}

5 Time-inconsistent Preferences in a General Equilibrium Model 69

5.1 Introduction . . . . . . . . . . . . . . . . . . 69

5.2 The Model . . . . . . . . . . . . . . . . . . . 72

5.3 Naïve Societies . . . . . . . . . . . . . . . . . . . 75

5.4 Sophisticated Societies . . . . . . . . . . . . . . . 79

5.5 Efficiency - the Myopic Case . . . . . . . . . . . . . . . . 83

5.5.1 Myopic Overall Pareto Efficiency . . . . . . . . . . . 84

5.5.2 Constrained Myopic Periodical Efficiency . . . . . . . . . 88

5.6 Efficiency - the Forward-looking Case . . . . . . . . . . . . . 89

5.6.1 Overall Pareto Efficiency . . . . . . . . . . . . 91

5.6.2 Constrained Periodical Efficiency . . . . . . . . . . 93

5.7 Conclusion . . . . . . . . . . . . . . . . . 96

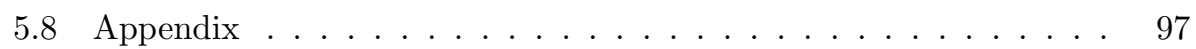

6 On the Completeness of Complete Markets 111

6.1 Introduction . . . . . . . . . . . . . . . . . 111

6.2 The Primitives of the Economy . . . . . . . . . . . . . . 115

6.3 Arrow-Debreu Markets . . . . . . . . . . . . . . . . . 118 
6.4 Sequentially Complete Markets . . . . . . . . . . . . . . . . . 119

6.4 .1 Naïve Behavior . . . . . . . . . . . . . . . . . . . 120

6.4.2 Sophisticated Behavior . . . . . . . . . . . . . . 121

6.4.3 Properties of Sequentially Complete Equilibria . . . . . . . 123

6.5 Complete Markets . . . . . . . . . . . . . . . . . . . 126

6.5.1 Naïve Behavior . . . . . . . . . . . . . . . . . . . 127

6.5.2 Sophisticated Behavior . . . . . . . . . . . 128

6.5.3 Properties of Complete Equilibria . . . . . . . . . . . . 130

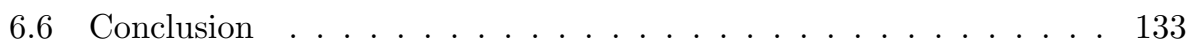

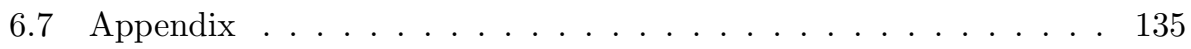

7 Arbitrage Opportunities in Frictionless Markets with Sophisticated Investors

Nederlandse Samenvatting (Summary in Dutch)

References

Author Index

Subject Index 



\section{ACKNOWLEDGMENTS}

Economics and mathematics were my favorite subjects at high school. It was a pleasure to find the Dutch econometrics study program, which combines both fields. Consequently, I began my studies in econometrics at Maastricht University in 1998. The first one and a half years of the curriculum were dominated by mathematics with very little economics. This caused me to consider switching over completely to economics. Then I became convinced of pursuing the subject of mathematical economics after taking the first mathematical economics course, which was taught by Jean-Jacques Herings. As a result of this course I began to consider pursuing a $\mathrm{PhD}$ degree in mathematical economics.

I have always had a great interest in psychology. Thus, in formulating my thesis topic with Jean-Jacques Herings, I expressed my wish to combine psychology with mathematical economics. We agreed that I would do research on general equilibrium and on decision theory, in particular intertemporal choice, where psychology is involved. We engaged Peter Wakker, an expert in decision theory to be my second promoter.

I would like to express my profound gratitude to Jean-Jacques Herings and Peter Wakker for the very pleasant cooperation. I appreciate the due diligence with which they attended to my work.

I would also like to thank Riccardo Welters for his support and the useful perspectives about $\mathrm{PhD}$ research that he shared with me during our walks to and from the railway station. I am grateful to Hans Peters for his support and career advise. Special thanks go to Arthur Attema and Han Bleichrodt for their cooperation in a few of the chapters of this dissertation. I am also grateful to Benjamin Arthur for correcting part of this thesis for the English language.

I thank my office mates Alexander Sebald and Jeroen van den Berg for the pleasant atmosphere they fostered in the office. I am grateful to all my other 
colleagues from Maastricht University.

Finally, special thanks go to my parents, Paul Rohde and Gea Rohde-Kotter, and my sister Ingrid Rohde for their constant support and endurance. 




\section{INTRODUCTION}

The majority of decisions that individuals make throughout their lives directly or indirectly concern tradeoffs over time. In our childhood going to school prevents us from immediately earning money by having a job, in return for an investment in our skills that will enable us to earn a higher income in the future. Many people save money or invest their money in stocks, which gives them fewer possibilities to spend money today in return for more possibilities to spend money in the future. Environmental issues mostly concern a tradeoff between beneficial consumption today and environmental damage in the future.

With the current ageing of the population, increasing attention is paid to our pensionsystem. The costs to society caused by the ageing of the population are huge (Feldstein, 2005). For governments it is an increasingly important issue how to design an optimal pension and social security system. Thus, it is important to understand the intertemporal tradeoffs a society and its members are willing to make. This thesis studies such intertemporal tradeoffs.

In economic and psychological models it is commonly assumed that individuals weigh immediate rewards and costs more than future ones. Thus, individuals discount future rewards and costs. Since Samuelson's (1937) introduction of constant discounted utility, this model has been a common assumption in economic analyses. Constant discounting, also known as exponential discounting, implies, under the additional assumption that preferences over costs and rewards do not change, that preferences over intertemporal tradeoffs do not change over time either. Under constant discounting, a decision made today will not have to be reconsidered in the future as long as there are no unforeseen events. Thus, constant discounting implies time-consistent preferences.

Strotz (1956) was the first to discuss an economic model with time-inconsistent preferences. Since the publication of his paper, many psychological and economic studies have found that individuals do not discount constantly (Frederick, Loewenstein, and O'Donoghue, 2002). Instead, hyperbolic discounting seems to be more 
accurate in describing the intertemporal tradeoffs that individuals make. When discounting is not constant, but, for instance, hyperbolic, preferences typically do change over time, i.e. preferences are time-inconsistent. Thus, the extensive findings of non-constant discounting require the dismissal of the assumption of time-consistent preferences in economic models if we want these models to capture reality. Today, much research is being done on this topic. The introduction of hyperbolic discounting in economic models has enabled economists to explain phenomena that could not be explained before, such as, for instance, a sharp drop in consumption at retirement and consumers reporting to save too little.

The introduction of time-inconsistent preferences in economic models not only implies that we have to redo the analyses done before. It also requires a reconsideration of the concepts that constitute economic models. It is, for instance, not obvious how traditional welfare concepts should be extended in the case of timeinconsistent preferences, as will be discussed later. In order to design and evaluate governmental policies and economic systems, welfare criteria are essential. Until now, only very little research has been done on defining appropriate welfare criteria when preferences change over time. In the second part of this thesis, welfare criteria are introduced and economic systems are analyzed under the assumption of time-inconsistent preferences. The development of more welfare criteria and further analysis of economic systems under the assumption of time-inconsistent preferences, should eventually enable governments and policy makers to, for instance, design appropriate pension systems that can deal with the ageing of the population.

The first part of this thesis deals with intertemporal preferences at an individual level. The second part deals with dynamic general equilibrium models and associated welfare criteria. The next three sections give an introduction to the first part, the link between the two parts, and an introduction to the second part.

\section{$1.1 \quad$ Intertemporal Choice}

The first part of this thesis considers intertemporal preferences of individuals. We consider the discounted utility model throughout this part. Under discounted utility, decision makers evaluate a stream of outcomes by first calculating the instant utility of every outcome if that outcome would be received immediately, then discounting that instant utility according to the timepoint when the outcome is received and, finally, summing all these discounted instant utilities. Thus, under discounted utility individuals can neglect timepoints where outcomes are 
unaffected when making a choice.

Discounted utility is commonly assumed in economics. The focus of much research in psychology and economics is on the type of discounting. Samuelson (1937) considered constant discounting, also known as exponential discounting. With constant discounting a preference between two streams of outcomes does not change if all outcomes in both streams are delayed by an equal period of time. Individuals exhibiting constant discounting are said to have constant impatience. From the perspective of today, their impatience, or discounting, between today and tomorrow is not different from the impatience between March 4, 2030 and March 5, 2030.

Much empirical evidence has demonstrated that humans and animals do not satisfy constant discounting (Frederick, Loewenstein, and O'Donoghue, 2002). Many studies have found that individuals are less impatient for the far future than for the near future, i.e. that they satisfy decreasing impatience. (Quasi-)hyperbolic discounting can capture decreasing impatience. Currently, quasi-hyperbolic discounting is becoming increasingly popular in applications. Hyperbolic and quasihyperbolic discounting are only two alternatives to constant discounting. Other alternatives could be thought of as well, but have not been considered in the literature. The main task in intertemporal choice today is to obtain a deeper understanding of the deviation from constant discounting and to develop appropriate alternatives. The first part of this thesis contributes to this task.

In order to obtain a good understanding of the deviation from constant discounting and to develop alternatives, it is important to first understand fully what constant discounting is. Koopmans $(1960,1972)$ gave a preference foundation of constant discounting that is very popular. A preference foundation is a set of conditions that fully characterize a preference model and that are easy to interpret and empirically verifiable. Thus, a preference foundation of constant discounting gives us a good insight into what constant discounting exactly implies and what it means empirically. Unfortunately, Koopmans' analysis contains some inaccuracies. Also, he does not allow for infinite economic growth in the infinite future, which is unnecessarily restrictive. Chapter 2 resolves these problems and provides, to the best of my knowledge, the first correct presentation of Koopmans' classical result.

Chapter 3 introduces a measure for the deviation from constant discounting. This measure, the hyperbolic factor, quantifies the degree of decreasing impatience and proneness to choice anomalies. The hyperbolic factor is easily measurable. Thus, it gives an easy tool to identify (groups of) individuals that are prone to choice anomalies. Moreover, the hyperbolic factor provides a simple test of hyper- 
bolic discounting: the hyperbolic factor is constant only in the case of hyperbolic discounting.

Chapter 4 introduces time-tradeoff (TTO) sequences to measure the discount function and the degree of decreasing or increasing impatience. These sequences are very easily obtained from individuals and can readily be analyzed using only paper and pencil. From TTO-sequences the degree of decreasing impatience and proneness to choice anomalies can immediately be inferred through simple graphs. By the nature of TTO-sequences, throughout the analysis, no assumptions or estimations of utility are needed. Almost all measurements of discounting in the literature did need to make assumptions about utilities. From TTO sequences, hyperbolic factors can readily be obtained. We also introduce other, heuristic, measures of decreasing impatience.

In an experiment TTO sequences are obtained from subjects. The results show that subjects indeed violate constant discounting. We find evidence in favor of increasing impatience, contrary to most of the literature, but in agreement with some other recent studies. Hyperbolic factors also provide evidence against the hyperbolic discount models popular in the literature. The findings suggest some new directions for theories of intertemporal choice.

\subsection{Intertemporal Behavior}

As argued before, and shown in the first part of this thesis, there is significant empirical evidence that intertemporal preferences of individuals do not satisfy constant discounting. If discounted utility holds and preferences over outcomes are constant over time, then a violation of constant discounting implies that individuals have time-inconsistent or dynamically inconsistent preferences. There is also other evidence that individuals have time-inconsistent preferences. Individuals with time-inconsistent preferences typically want to reconsider choices they made in the past, even if nothing unforeseen has happened. Therefore, individuals who realize that their preferences are time-inconsistent want to make costly precommitments in order to avoid a future reconsideration of plans. There is indeed evidence for individuals engaging in costly pre-commitment (Schelling, 1984).

A classical example of an individual engaging in pre-commitment is Odysseus (also called Ulysses) from The Odyssey written by Homer. On his ship, Odysseus had to pass the island with the Sirens who could sing so beautifully that everybody's attention would be drawn to them, so that he would risk shipwreck against the rocks. Odysseus, who foresaw this risk, had his sailors put wax in their ears 
and had them tie him to the mast, so that he could still hear the sirens without causing any damage.

For individuals with time-inconsistent preferences, the preferences concerning future periods basically change over time. Individuals with changing preferences can behave in several ways, depending on their self-knowledge. Naïve individuals do not realize that their preferences will change over time and will be surprised by their unanticipated preferences every period and want to reconsider their choices and update their plans (Pollak, 1968). Sophisticated individuals, like Odysseus, correctly predict their future preferences and either want to pre-commit themselves or only make plans that they expect to stick to. Partly sophisticated individuals do anticipate that their preferences will change over time, but predict their future preferences incorrectly. Thus, they behave similarly as sophisticated households by pre-committing and by making consistent plans, but are nevertheless surprised by their preferences every period.

\subsection{General Equilibrium}

The second part of this thesis analyzes the behavior of naïve and (partly) sophisticated individuals at an aggregate level in general equilibrium models. A general equilibrium model consists of consumers and producers who trade goods and services, called commodities. Consumers initially own a certain amount, or endowment, of commodities. Producers have a technology that can use some commodities as inputs to produce other or the same commodities as output. The producers or firms are owned by consumers, who own a given amount of shares of the firms. Consumers have preferences over consumption. Their objective is to satisfy their preferences as much as possible by trading commodities. Prices determine the ratios in which commodities can be exchanged between consumers and producers. The endowments and the shares in the profits that producers make determine the wealth of consumers. Consumers will typically want to increase their wealth in order to be able to buy more commodities. Thus, they will typically force producers to act in such a way that the wealth of consumers is maximized. Consumption, production and prices then constitute an equilibrium if there is no excess demand or excess supply of commodities. Markets are perfectly competitive if there are so many producers and consumers that one consumer or producer on his own cannot influence prices. Then consumers and producers take prices as given and the equilibrium is called a competitive equilibrium.

The most common welfare criterion used in general equilibrium theory, is 
Pareto efficiency. An allocation of commodities is said to be Pareto efficient if no consumer can be made better off without making another consumer worse off.

Traditional general equilibrium models assume that preferences of individuals do not change over time. It can then be shown that when markets are complete, i.e. when individuals can trade all possible future commodities already today by making contracts, a competitive equilibrium always exists under rather weak assumptions. The First Fundamental Theorem of Welfare Economics holds that every competitive equilibrium allocation in complete markets is Pareto efficient. The Second Fundamental Theorem of Welfare Economics shows that every Pareto efficient allocation can be attained as a competitive equilibrium in complete markets by appropriate redistributions of endowments. Thus, these theorems show that, apart from redistributions of endowments, government intervention may be welfare improving only in the case of externalities, market power, asymmetric information or restrictions on trading future commodities.

In the derivation of these results it has always been assumed that preferences do not change over time. When preferences do change over time it is not clear whether these theorems still hold. It could very well be that when preferences change over time, the role of the government might be more active also when markets are complete. Actually, it is even no longer clear how a competitive equilibrium and efficiency should be defined. For efficiency, for instance, the question arises which preferences should be considered in the efficiency concepts, the current preferences, the future preferences, or both. The second part of this thesis introduces changing preferences in general equilibrium models. Preferences are very general and do not need to satisfy discounted utility. Since the modelling is quite complicated, we only study exchange economies, i.e. economies without production. We will refer to consumers as households.

Chapter 5 introduces equilibrium- and welfare- concepts in general equilibrium models where households cannot trade future commodities already today. We are the first to introduce general changing preferences in a full-fledged general equilibrium model. We introduce new equilibrium concepts, show which issues arise in defining welfare criteria, and show how these issues can be solved. We further establish the existence of equilibria and show that some efficiency concepts are satisfied in equilibrium, and others are not.

Chapter 6 analyzes the notion of completeness of markets. Markets are complete when all possible contingent commodities can be traded. A contingent commodity is a commodity for which one pays today and which is delivered in a future period, contingent on the realization of a particular event. Markets are sequentially complete when in every period all one-period ahead contingent commodities 
and commodities on spot markets can be traded. The First and Second Theorems of Welfare Economics used to hold under the assumption of complete markets and sequentially complete markets. Actually, the two market structures used to yield the same consumption in equilibrium. When preferences change over time it is no longer the case that the two markets structures yield the same allocations when individuals are not fully sophisticated, which is shown in Chapter 6. Thus, as soon as individuals are not fully sophisticated the degree of completeness of markets matters.

Chapter 7 shows by means of an example that, in equilibrium, there can exist assets that cost nothing today and that yield a positive income in future periods. This example gives some feeling for the consequences of the introduction of changing preferences in economic models. Most economists would think that all individuals want to increase their future income as much as possible. The example shows that sophisticated individuals might not want to increase future income. Such a sophisticated individual knows that tomorrow he will spend his income in a way that he does not like today. As a result, arbitrage opportunities might not be taken away by the individuals. 



\section{Part I}

\section{Intertemporal Choice}





\section{Koopmans' Constant Discounting: A Simplification AND AN Extension to INCORPORATE ECONOMIC GROWTH}

Koopmans provided a well-known preference foundation for constant discounted utility, the most widely used model for intertemporal optimization. There were, however, some technical problems in his analysis; in particular, there was an unforeseen implication of bounded utility. Some partial resolutions have been suggested in the literature. This chapter ${ }^{1}$ completely resolves the problems mentioned, with complete flexibility regarding the utility functions that can be used and the conceivable economic growth. It, thus, provides a complete and accessible preference foundation of constant discounted utility, and clarifies the appeal of Koopmans' intuitive axioms.

\subsection{Introduction}

Preference foundations give conditions for preferences that are necessary and sufficient for the applicability of some theoretical choice model, such as the constant discounted utility model for intertemporal consumption programs, the topic of this chapter. Preference foundations, thus, express the empirical content of a model directly in terms of observables. One of the most appealing and well-known preference foundations is Koopmans' $(1960,1972)$ foundation for constant discounted utility. This model, originally introduced by Samuelson (1937), is often considered normatively warranted. It is the almost exclusively used model in prescriptive applications of intertemporal optimization and in policy recommendations. For such applications, preference foundations serve to justify (or criticize) the appropriateness of the model used.

\footnotetext{
${ }^{1}$ This chapter is based on Bleichrodt, Rohde, and Wakker (2005)
} 
In descriptive applications, preference foundations serve to demonstrate how a model can be falsified or corroborated through observed choices. Indeed, many recent empirical studies have examined, and mostly falsified, the preference conditions of constant discounted utility. Constant discounting is then commonly taken as an initial benchmark, the proper variation of which is to be developed (Frederick, Loewenstein, and O'Donoghue, 2002; Laibson, 1997).

As we will explain later, the intuitive part of Koopmans' preference foundation is exceptionally appealing and efficient. While his chapter has been widely cited, the appeal and efficiency of his intuitive axioms have not always been fully understood. These axioms are, unfortunately, obscured by technical digressions and some inaccuracies in the analysis. It cannot always be decided with certainty what technical conditions Koopmans assumed; see the appendix. Koopmans derived his result only for bounded consumption programs (Koopmans, 1960, Postulate P5; Koopmans, 1972, Postulate P5'), but speculated on extensions to other programs if his axiom P5/P5' is dropped (Koopmans, 1960, Section 10*; Koopmans, 1972, Section $\left.6^{*}\right)$. However, even if Koopmans' P5/P5' is dropped, then his remaining conditions still imply that utility must be bounded (see Example 2.4 in the appendix). Most utility functions considered in the literature, such as CRRA and CARA utilities (Merton, 1971), are unbounded from at least one side if no restrictions are imposed on their domain. Koopmans' utility bounds, therefore, impose undesirable restrictions on the outcomes for such cases. Especially for unbounded time horizons it is undesirable to exclude continuing economic growth. It is pessimistic to a priori impose upper bounds on the utility levels attainable for all the future (see Koopmans, 1972, following Postulate P5').

Several alternative axiomatizations of constant discounted utility have been given in the literature. Authors usually axiomatized generalizations of constant discounted utility, and derived axiomatizations of constant discounted utility as a corollary and a by-product. Also Koopmans (1960) himself obtained constant discounted utility only as a by-product. His main interest concerned more complex models of general recursive intertemporal aggregations that need not be additively separable over time, and his primary purpose was to derive impatience from continuity conditions. This chapter restricts attention to constant discounted utility, and presents preference axioms that are more general and accessible than preceding ones for this model.

We relax Koopmans' topological assumptions so as to avoid the undesirable implication of bounded utility, and completely solve the problems of unbounded utility. That is, first, we allow for every utility function, and, second, our domain has maximal flexibility concerning the programs considered. For every utility 
function, also if unbounded, we are free to incorporate (or exclude) every program that generates unbounded utility in the future as long as its discounted utility is defined and finite.

Koopmans' characterization of constant discounted utility is one of the most appealing and important preference foundations known today. By resolving his technical problems and simplifying the presentation, we hope to make the appeal and efficiency of Koopmans' intuitive axioms more accessible.

\subsection{The Result}

A (consumption) program $x=\left(x_{1}, x_{2}, \ldots\right)$ yields consumption $x_{t}$ in period $t \in \mathbb{N}$, where $X=\mathbb{R}_{+}^{m}$ is the set of all conceivable consumptions. ${ }^{2}$ Preferences over consumption programs are denoted by $\succcurlyeq$, with $\succ, \sim, \preccurlyeq$, and $\prec$ as usual. Our main result will allow for great flexibility regarding the domain of the programs considered, denoted $F$ hereafter. We will characterize the conditions required for this $F$ later on. The main reason for the complications in Koopmans' analysis is that he did not allow for a restricted domain of programs on which the preference relation is defined and should satisfy completeness and various other conditions. With unbounded utility, there can be programs with infinite or undefined discounted utility, and imposing completeness in combination with other preference conditions on such programs is problematic.

A classical illustration of the above problems is Savage (1954), who similarly imposed completeness and other axioms on all programs ("acts" in his model). An unforeseen implication, discovered by Fishburn, is that Savage's axioms imply that utility must be bounded (Savage, 1972, 2nd edition, footnote on p. 80). Koopmans' axioms similarly have this unforeseen implication (see Example 2.4). Koopmans, Diamond, and Williamson (1964, Section $8^{*}$ ) speculated on such problems and explicitly restricted their analysis to bounded utility. Koopmans (1972), however, defined a preference relation and its conditions on the full domain again and without boundedness restriction, adding the latter only for particular results, and speculating on dropping it.

Our way to handle unbounded utility builds on Harvey (1986) and Wakker (1993a). As these authors did, we avoid the problems mentioned above by imposing preferences and their conditions only on restricted domains of programs, entirely defined in terms of observables. Constant discounted utility holds on a domain

\footnotetext{
${ }^{2}$ All results of this chapter remain valid if $X$ is a topologically separable arc-connected topological space; see the appendix. It can, for instance, be any convex subset of $\mathbb{R}^{m}$, or a set of nonquantified health states.
} 
of programs if there exist a utility function $u: X \rightarrow \mathbb{R}$ and a discount factor $0<\rho<1$ such that every consumption program $x$ in the domain is evaluated through the well-defined finite

$$
\sum_{t=1}^{\infty} \rho^{t-1} u\left(x_{t}\right) .
$$

This summation is called the discounted utility of $x$. A program is preferred if and only if it is evaluated higher. The evaluation implies the common assumption of weak ordering of $\succcurlyeq$, meaning that $\succcurlyeq$ is complete $(x \succcurlyeq y$ or $y \succcurlyeq x$ for all $x, y$, possibly both) and transitive. Note that many papers that do not assume additive separability over time do not have a concept such as $u$ above, and often use the term utility for what we call discounted utility. In our set-up it is most convenient to let utility designate the "instant" utility $u$.

Before defining the axioms, we introduce some preparatory notation. For a program $x=\left(x_{1}, x_{2}, \ldots\right)$ and a consumption $\alpha, \alpha x$ denotes the program $\left(\alpha, x_{1}, x_{2}, \ldots\right)$ where the first consumption is $\alpha$ and then the consumptions of $x$ follow, all delayed by one period. The procedure can be repeated, as in $\alpha \beta x=\left(\alpha, \beta, x_{1}, x_{2}, \ldots\right)$, etc.

Preferences over consumptions agree with preferences over constant programs in the sense that $\alpha \succcurlyeq \beta$ if and only if $(\alpha, \alpha, \ldots) \succcurlyeq(\beta, \beta, \ldots)$. Koopmans (1972, p. 84) discussed (a small variation of) the following condition. Monotonicity holds if $x \succcurlyeq y$ whenever $x_{t} \succcurlyeq y_{t}$ for all $t$, with strict preference $x \succ y$ whenever $x_{t} \succ y_{t}$ for some $t$. In our result this condition need not be imposed because it is implied by the other conditions, mainly stationarity. To avoid triviality, we will, like Koopmans (1960, Postulate 2), assume that period 1 is sensitive, i.e. $\alpha x \succ \beta x$ for some $\alpha, \beta, x$.

The most interesting axioms in preference foundations are the intuitive axioms characteristic of the model considered. Koopmans assumed, essentially, the following two intuitive axioms. Initial-tradeoff independence holds if

$$
\alpha \beta x \succcurlyeq \gamma \delta x \text { if and only if } \alpha \beta y \succcurlyeq \gamma \delta y
$$

for all programs $x, y$ and all consumptions $\alpha, \beta, \gamma, \delta$. That is, the tradeoffs between today and tomorrow are not affected by future consumption. The condition amounts to separability of the first two periods. A set of periods is separable if preferences over consumptions in these periods, while keeping consumptions in other periods fixed, are independent of the level at which the other consumptions are kept fixed.

Stationarity holds if

$$
\alpha x \succcurlyeq \alpha y \text { if and only if } x \succcurlyeq y
$$


for all programs $x, y$ and all consumptions $\alpha$. That is, a preference is not affected if a common first consumption is dropped, and the timing of all other consumptions is advanced by one period. By repeated application, it implies that for a preference between two programs all initial periods with common consumption can be dropped, and the first period of different consumption can be taken as the initial period. It is amazing that the two simple conditions just defined, in the presence of some other common assumptions, suffice to imply the whole time-additive constant discounted utility model, as Theorem 2.1 will demonstrate.

Koopmans formulated his intuitive axioms equivalently but slightly differently, with for instance stationarity imposed only for one initial consumption $\alpha$ and then separability of $\{2,3, \ldots\}$ added, which is equivalent to our stationarity imposed for all initial consumptions $\alpha$. The difficulties in his analysis are due to the technical conditions. In his model as well as in ours, programs are infinite-dimensional objects, and then topological considerations can be complex. We avoid such complexities by imposing topological conditions (continuity) only on finite-dimensional subspaces. For two further implications of infinite-dimensional continuity that are used in proofs of other papers, constant-equivalence and tail-robustness (defined later), it will be both more appealing and more general to state these as explicit axioms, rather than to derive them from stronger infinite-dimensional continuities.

An ultimately constant program $x$ is such that $\alpha=x_{t}=x_{t+1}=\cdots$ for all $t>T$, for some consumption $\alpha$ and some period $T$. By $x_{T} \alpha$, for some general program $x$, period $T$, and consumption $\alpha$, we denote the ultimately constant program $\left(x_{1}, \ldots, x_{T}, \alpha, \alpha, \ldots\right)$. For each period $T, X_{T}$ is the set of ultimately constant programs of the form $x_{T} \alpha$, i.e., all ultimately constant programs that are constant over all $t>T . X_{T}$ can be considered a $T+1$ dimensional product space, specified by $T+1$ tuples $\left(x_{1}, \ldots, x_{T}, \alpha\right)$. Ultimate-continuity holds if $\succcurlyeq$ is continuous on each set $X_{T}$.

To extend the discounted-utility evaluation to unbounded programs, we, contrary to Koopmans, do not impose a preference relation on all programs, but only on those that will have finite discounted utility. To identify those programs, we have to solve a mathematical problem that has hampered many papers dealing with infinite-dimensional evaluations. A typical example of the problem mentioned is the derivation of subjective expected utility in DeGroot (1970, Chapter 7). Having derived the evaluation on bounded programs ("acts"), he explicitly used utility and the expected utility functional to define the domain of all programs with finite expected utility (denoted $\mathcal{P}_{E}$ in his Section 7.10), and then went on to establish the preference axiomatization of expected utility in terms of preference conditions on this extended domain. This procedure is undesirable because utility and expected 
utility are theoretical constructs and are related to observables only in complex ways. Hence, they should not be used explicitly in preference foundations. In fact, if expected utility can be used explicitly in the definition of the domain and in preference conditions, then its preference axiomatization becomes a tautology because we can simply state expected utility maximization directly as a preference axiom. A similar problem as in DeGroot's analysis arose in Hübner and Suck's (1993) extension of Koopmans' constant discounted utility to unbounded programs. They used a condition about the interior of circles of convergence that explicitly uses both the discount factor and the utility function.

The problem to be solved is that we should find preference conditions that identify the programs with bounded discounted utility, but that are stated entirely in terms of observables (preferences) without any explicit use of utility or discounted utility. Two studies partially resolved this problem, by defining a particular subset of programs that have finite discounted utility in terms of preference conditions. First, Dolmas (1995) took a fixed program $\omega \in \mathbb{R}_{++}^{m}$, and considered all programs $x$ for which there exists $\lambda>0$ such that $\left|x_{t}\right| \leq\left|\lambda \omega_{t}\right|$ for all $t$. Thus he partially relaxed the boundedness restriction. He imposed continuity with respect to a modified supnorm that made his space of programs homeomorphic (equivalent in a topological sense) to the set of all bounded programs, and derived discounted utility on this extended domain from a strengthening of Koopmans' axioms. Second, Streufert (1990) considered only the programs that can be generated by a particular production function, which can again relax the boundedness restriction partially. He introduced a bi-convergence continuity condition in terms of the production function, and derived discounted utility on this extended domain from a strengthening of Koopmans' axioms. Neither of these works specified the exact restrictive nature of their preference conditions through necessity results. Both allowed for some unbounded programs and utility functions but not all, and it is not clear what exactly the restrictions on $u$ are in terms of $\omega$ or Streufert's production function. An advantage of these approaches is that the domain of preference can be defined prior to any consideration of the preference relation, whereas in our approach it will refer to the preference relation.

Conditions that can identify all programs with finite discounted utility, whatever the (unbounded) utility function, were used by Harvey (1986) for the present context of summation over discrete periods, and by Wakker (1993a) for integrals over general spaces for the context of decision under uncertainty. The approach of this chapter will be a mix of these two approaches, using two preference conditions introduced next.

A program $x$ satisfies constant-equivalence on a domain if there exists an equi- 
valent constant program in that domain. This condition was derived from topological conditions in Diamond (1965, Lemma on p. 172), Harvey (1986, p. 1136 second para) and in Koopmans (1960, Eq. 17 and Section 10). Second, a program $x$ is tail robust if, for all outcomes $\beta$ : if $x \succ \beta(x \prec \beta)$ then there exists a $t$ such that $x_{T} \beta \succ \beta\left(x_{T} \beta \prec \beta\right)$ for all $T \geq t$. In words, a sufficiently remote future does not affect preference much. The two conditions just defined deliver, in terms of preference conditions, the domain of programs searched for. A program will turn out to have well-defined finite discounted utility if and only if it satisfies constantequivalence and tail robustness. These definitions modify the $C^{*}$ definition of Harvey (1986) and truncation-robustness of Wakker (1993a).

In the characterizing Statement (ii) in the following theorem, (a) states usual preference conditions, with continuity only in a simple finite-dimensional version, (b) defines the domain of programs with finite discounted utility, and (c) gives Koopmans' intuitive conditions. The uniqueness up to unit and level in the following theorem means that any constant can be added to utility, and it can be multiplied by any positive number.

Theorem 2.1 Let $\succcurlyeq$ be defined on a domain $F$ of programs that contains all ultimately constant programs. Then the following two statements are equivalent.

(i) Constant discounted utility holds on $F$, where the utility function is continuous and not constant, and all programs have finite discounted utility.

(ii) $\succcurlyeq$ satisfies

(a) weak ordering, sensitivity of the first period, and ultimate-continuity;

(b) constant-equivalence and tail robustness for each program in F;

(c) initial-tradeoff independence and stationarity.

Furthermore, the discount factor in Statement (i) is unique, and utility is unique up to unit and level.

Some of the above conditions can be relaxed on particular domains. A program $x$ is bounded if there exist consumptions $\mu, \nu$ such that $\mu \succcurlyeq x_{t} \succcurlyeq \nu$ for all $t$.

Observation 2.2 In Theorem 2.1, monotonicity is implied by the other conditions. If $F$ contains only ultimately constant programs, then constant-equivalence can be dropped and tail robustness can be replaced by monotonicity. Tail robustness can also be replaced by monotonicity if F contains only bounded programs. 


\subsection{Related Literature}

The efficiency of Koopmans' intuitive axioms, in particular stationarity, is exceptional. To illustrate it, we sketch the proof of Theorem 2.1. First, by repeated application, stationarity implies independence of preference from every common first part of programs, not just if related to the first period. Then, because of stationarity, separability of the first two periods implies separability of every pair of consecutive periods. Stationarity also implies separability of the "tail of periods" $\{2,3, \ldots\}$, and then of every tail of periods $\{t, t+1, \ldots\}$. These separabilities together imply complete separability of all periods (Gorman, 1968; Streufert, 1995) and, hence, an additively separable evaluation

$$
x \mapsto V_{1}\left(x_{1}\right)+V_{2}\left(x_{2}\right)+\cdots,
$$

given appropriate continuity. Other papers in the literature used stronger separability than of merely $\{1,2\}$, as we did; see Table 2.1 .

Stationarity further implies that preferences over consumptions are the same in each period, so that the component-functions are ordinally the same:

$$
V_{t}=f_{t}\left(V_{1}\right) \text { for a strictly increasing } f_{t}
$$

Several papers in the literature used conditions other than stationarity to obtain Eq. 2.5; see Table 2.1. Stationarity further implies that all functions evaluating consumption in different periods order differences in the same way, so that they can be taken proportional, leading to the general period-dependent discounting

$$
V_{t}=\rho_{t} V_{t-1} \text { for } \rho_{t}>0 \text {. }
$$

Table 2.1 indicates that several papers used conditions other than stationarity to obtain this implication. Stationarity implies, furthermore, constant discounting with $\rho_{t}=\rho$ independent of $t$. Several papers first derived general, perioddependent, discounting as in Eq. 2.6 from separate preference conditions, and then could use weaker versions of stationarity to obtain constant discounting. See Harvey $(1986,1995$, (partial) absolute timing preference), Bleichrodt and Gafni (1996, p. 53), Meyer (1976, p. 480), and Wakker (1989, the $\$ \alpha$ condition in Statement (ii), p. 88). The partial results in the last column of Table 2.1, on the possibility of unbounded utility, have been discussed in Section 2.2.

The consumption sets $X$ in the literature were mostly less general than in this chapter. They were: $\mathbb{R}_{+}^{m}$ in Dolmas (1995); an open interval in Meyer (1976) and Harvey $(1986,1995)$; a connected subset of $\mathbb{R}^{m}$ in Koopmans (1960, 1972). For 


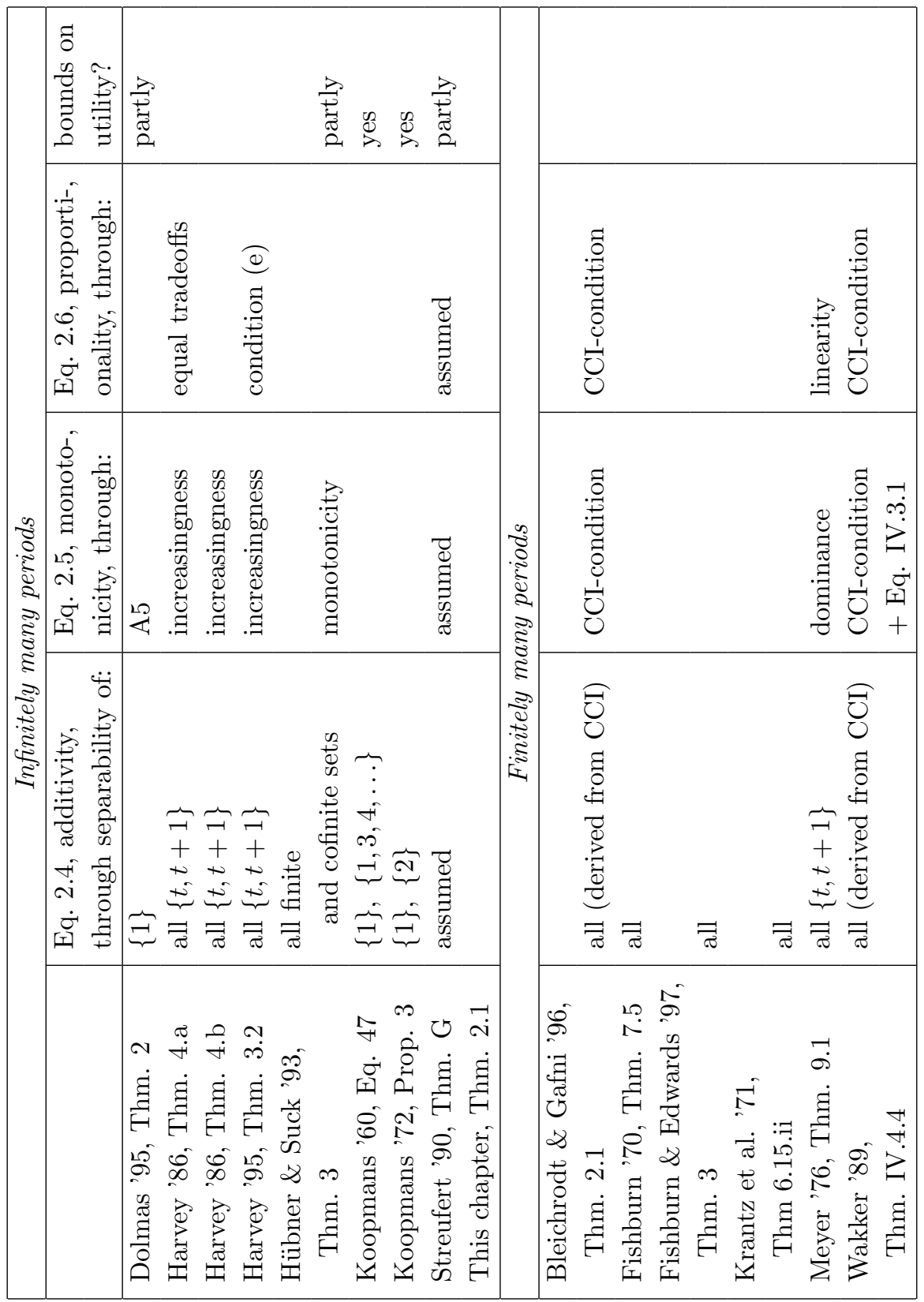

Table 2.1: Extra nontechnical conditions besides those in Theorem 2.1 (stationarity and separability of $\{1,2\}$ ) assumed in the literature for constant discounted utility. 
finitely many periods, some papers considered more general consumption sets, but then could not use Gorman's (1968) theorem and had to assume full-force separability. Thus, the consumption set was connected in Fishburn and Edwards (1997), Bleichrodt and Gafni (1996), and Wakker (1989). The consumption set only had to satisfy a solvability condition, which is more general than any of the restrictions mentioned so far, in Krantz et al. (1971) for finitely many periods and in Hübner and Suck (1993) for infinitely many periods. Some further restrictions are linear utility in Harvey (1995, Theorem 3.2) and Meyer (1976). Fishburn and Edwards (1997) did allow for infinitely many periods, but only compared programs that differ in at most finitely many periods so that, essentially, only finite-dimensional considerations arise. Also the well-known Fishburn and Rubinstein (1982) considered an infinite set of conceivable periods, but restricted attention to single consumption. Then no issues of intertemporal separability or diverging sums of utility will arise, and this study is not easily compared to the other models considered here.

The above papers, scattered in journals in economics, management science, and psychology, and written when internet was not yet available, obtained their extensions of Koopmans' theorem independently. With the exception of Fisburn and Edwards (1997) referring to Harvey (1995) and Streufert (1990), none of the above papers referred to any of the other contributions for infinitely many periods other than Koopmans'. It is remarkable that mere separability of $\{1,2\}$ plus stationarity imply all the conditions of the works listed in Table 2.1. In this comparison we should emphasize that the other papers were not designed to optimally characterize discounted utility. They usually sought to generalize Koopmans' model, and constant discounted utility was then obtained as a byproduct.

Theorem 2.1 allows for great flexibility regarding the domain $F$. The only restriction is that $F$ should contain all ultimately constant programs. It can, for instance, contain all programs with finite discounted utility, as in Harvey (1986), but it can also contain any arbitrary subset of the latter given the restriction mentioned. For each program $x$ that is not ultimately constant, we can check if it can be added to the preference domain by verifying Statement (ii.b), i.e. constant-equivalence and tail robustness. These conditions involve, besides $x$, only ultimately constant programs. No restriction is imposed on the utility function $u$. 


\subsection{Conclusion}

Many generalizations of Koopmans' constant discounted utility have been developed, and preference foundations have been given. Koopmans' model was then sometimes obtained as a corollary of the generalization. No paper has, however, focused on the constant discounted utility model itself. Consequently, for this model, the by far most used model in applications, and a common benchmark for alternative models, no clean and efficient preference foundation exists today. This chapter has provided such.

We are not aware of any preference condition in the literature that is so appealing and simple to comprehend, and at the same time so powerful in its implications, as Koopmans' stationarity. We hope that this chapter can help to clarify and popularize Koopmans' intuitive axioms.

\subsection{Appendix A. Proofs}

The following example prepares for the proof. It shows that tail robustness cannot be omitted in Theorem 2.1. It may clarify Koopmans' (1972) discussion of his monotonicity postulates P5 and P5'.

Example 2.3 $F$ consists of all ultimately constant programs. Evaluations are through

$$
\left(x_{1}, \ldots, x_{T}, \alpha, \alpha, \ldots\right) \mapsto \sum_{i=1}^{T} \rho^{i-1} u\left(x_{i}\right)+\rho^{T} u(\alpha) /(1-\rho)
$$

with $u$ continuous and not constant, and $\rho>1$. The evaluation is well-defined, because, if $x_{T+1}=\alpha$, then

$$
\sum_{i=1}^{T} \rho^{i-1} u\left(x_{i}\right)+\rho^{T} u(\alpha) /(1-\rho)=\sum_{i=1}^{T+1} \rho^{i-1} u\left(x_{i}\right)+\rho^{T+1} u(\alpha) /(1-\rho),
$$

etc. All conditions of Statement (ii) in Theorem 2.1, except tail robustness, are satisfied. Besides tail robustness, also monotonicity and impatience (preference for early receipt of outcomes with higher $u$ value) are violated. We obtain an evaluation of $\alpha$ equal to $u(\alpha)+\rho u(\alpha)+\rho^{2} u(\alpha) /(1-\rho)=u(\alpha) /(1-\rho)$. It is not increasing, but decreasing, in $u(\alpha)$ for $\rho>1$. Preferences satisfy a weaker monotonicity condition, i.e. finite monotonicity, which means that replacing any finite number of consumptions $x_{t}$ by other consumptions with higher $u$ value always improves the program. By sensitivity of period 1, $u$ is not constant; and there are 
$u(\gamma)>u(\beta)$. Then $\gamma \prec \beta$. This preference and $\gamma x \succ \beta x$ violate monotonicity. It is like preferring cake to bread for any finite number of days, but preferring bread to cake for an infinite lifetime. Tail robustness would require that $\gamma_{T} \beta \prec \beta$ should hold for all $T$ sufficiently large. However, by finite monotonicity, $\gamma_{T} \beta \succ \beta$ for all $T$.

Even if utility of consumption is bounded, the evaluation of programs is not (cf. $\gamma_{T} \beta$ for $T$ increasing). There still does not exist a best program, so that Koopmans' (1972) Postulate P5' is still violated.

This example is an alternative to the example of Burness (1976, p. 505), who showed that the very existence of a utility indicator need not imply impatience. Our example revealed a more fundamental problem, i.e. a violation of monotonicity. By imposing the supremum norm, all conditions of Burness (1976) are satisfied.

Proof of Theorem 2.1. The following proof and, consequently, Theorem 2.1 , are valid for every topologically separable ${ }^{3}$ and arc-connected consumption space $X$. For each $x, D U(x)=\sum_{t=1}^{\infty} \rho^{t-1} u\left(x_{t}\right)$ whenever defined. First assume that Statement (i) holds. Tail robustness follows because of convergence of the summations in $D U(x)$. There exists a constant-equivalent for each $x$, with utility strictly between $\limsup \left(u\left(x_{t}\right)\right)$ and $\liminf \left(u\left(x_{t}\right)\right)$ if these two are different, mainly because of connectedness of $u(X)$. All other conditions in Statement (ii) follow easily.

For the reversed implication, we assume henceforth that Statement (ii) holds. We fix an arbitrary outcome $\theta$ throughout, at which many functions below will be normalized to be 0 . Initial-tradeoff independence amounts to separability of the set of periods $\{1,2\}$, with a preference $\alpha \beta x \succcurlyeq \gamma \delta x$ independent of $x$. By stationarity, it implies that a preference $\mu \alpha \beta x \succcurlyeq \mu \gamma \delta x$ is independent of $\mu$ and $x$, i.e. $\{2,3\}$ is separable. Similarly, by repeated application of stationarity, separability of all sets $\{i, i+1\}$ follows. Stationarity implies that a preference $\alpha x \succcurlyeq \alpha y$ is the same as between $x$ and $y$ and, hence, is independent of $\alpha$, so that separability of $\{2,3, \ldots\}$ follows. Similarly as above, stationarity then implies separability of $\{3,4, \ldots\}$, and then of all "tail" sets $\{i, i+1, \ldots\}$. Similarly, sensitivity of period 1 and stationarity imply sensitivity of all periods $t$.

Consider a period $T>1$. By the separabilities just established, and the other conditions such as sensitivity of at least three periods (in fact all) and continuity, Gorman (1968) implies that we have complete separability (i.e., separability of all

\footnotetext{
${ }^{3}$ We add the adjective "topological" throughout to distinguish this condition, not defined here, from the separability preference condition.
} 
subsets of components) over every $T+1$ dimensional space $X_{T}$, and an additive evaluation

$$
\left(x_{1}, \ldots, x_{T}, \alpha, \alpha, \ldots\right) \mapsto V_{1, T}\left(x_{1}\right)+\cdots+V_{T, T}\left(x_{T}\right)+R_{T}(\alpha)
$$

on each such set. We may set $V_{j, T}(\theta)=0=R_{T}(\theta)$ for all $j$. The function

$\left(x_{1}, \ldots, x_{T}, \alpha, \alpha, \ldots\right) \mapsto V_{1, T+1}\left(x_{1}\right)+\cdots+V_{T, T+1}\left(x_{T}\right)+V_{T+1, T+1}(\alpha)+R_{T+1}(\alpha)$,

obtained from $X_{T+1}$, is an alternative additive evaluation over $X_{T}$. By the usual uniqueness results of additive evaluations, we may, inductively with respect to $T$, set

for all $T$, and all $j \leq T, V_{j, T+1}=V_{j, T}$ and $V_{T+1, T+1}(\alpha)+R_{T+1}(\alpha)=R_{T}(\alpha)$.

From the first equality it follows that $V_{j, T}$ is independent of $T$ for $j \leq T$, and we can drop the subscript $T$.

By stationarity and consideration of programs $\gamma x$ for a fixed $\gamma, V_{2}\left(x_{1}\right)+\ldots+$ $V_{T+1}\left(x_{T}\right)+R_{T+1}(\alpha)$ evaluates the same preferences over $X_{T}$ as does $V_{1}\left(x_{1}\right)+$ $\ldots+V_{T}\left(x_{T}\right)+R_{T}(\alpha)$. By the usual uniqueness results, there exists a $\rho_{T}>0$ such that $V_{j+1}=\rho_{T} V_{j}$ for all $j \leq T$ and

$$
R_{T+1}=\rho_{T} R_{T} .
$$

$\rho_{T}=V_{2} / V_{1}$ is independent of $T$ because $V_{1}$ and $V_{2}$ are, and we drop the subscript $T$. Writing $u=V_{1}$ and $R=R_{2} / \rho^{2}$, we have obtained an evaluation

$$
\left(x_{1}, \ldots, x_{T}, \alpha, \alpha, \ldots\right) \mapsto \sum_{i=1}^{T} \rho^{i-1} u\left(x_{i}\right)+\rho^{T} R(\alpha) .
$$

Note that consumptions are ordered the same for every period, which comprises most of monotonicity. The last equality in Eq. 2.9 implies that $\rho^{T} R(\alpha)=\rho^{T} u(\alpha)+$ $\rho^{T+1} R(\alpha)$, or

$$
R(\alpha)=u(\alpha)+\rho R(\alpha) .
$$

$\rho=1$ cannot be: By Eq. 2.12, then $u(\alpha)=0$ for all $\alpha$. Constantness of $u$ violates sensitivity of period 1 , and $\rho=1$ cannot be indeed. Hence,

$$
R=u /(1-\rho) .
$$

$\rho>1$ cannot be either because it violates tail robustness (and monotonicity); see Example 2.3. We conclude that, besides $0<\rho$, also $\rho<1$. Eq. 2.13 implies 
$R(\alpha)=\sum_{i=1}^{\infty} \rho^{i-1} u(\alpha)$. Substituting this in Eq. 2.11 yields discounted utility for the ultimately constant programs.

To extend the evaluation to general, possibly unbounded, programs, consider a general program $x$ and its constant-equivalent $\alpha$. If there exists a consumption $\beta$ with $x \sim \alpha \succ \beta$ then, by tail robustness, $x_{T} \beta \succ \beta$ for all $T$ sufficiently large. By the evaluation for ultimately constant programs, $D U\left(x_{T} \beta\right)>D U(\beta)$ for all $T$ sufficiently large. Hence, $\liminf _{T}\left(\sum_{t=1}^{T} \rho^{t-1} u\left(x_{t}\right)\right) \geq D U(\beta)$. Because this holds for all $\beta \prec \alpha$, the liminf also is not less than $D U(\alpha)$. If there exists no $\beta$ as above, then $\alpha$ is the worst consumption, and by the $D U$ representation and its implication of monotonicity on the ultimately constant programs, the above liminf again is not less than $D U(\alpha)$. Similarly, $\limsup _{T} \sum_{t=1}^{T}\left(\rho^{t-1} u\left(x_{t}\right)\right)$ is not more than $D U(\alpha)$. It follows that $D U(x)=D U(\alpha)$ and, hence, $D U(x)$ evaluates $x$.

Note that we nowhere made any assumption about which of the not ultimately constant programs are contained in $F$, and complete flexibility of domain has been maintained. By standard uniqueness results for additive evaluations (Gorman, 1968), the additive evaluations such as in Eq. 2.8 have the functions $V_{j, T}=\rho^{j-1} u$ unique up to level and common unit. This implies the uniquess result regarding $u$ and $\rho=\left(V_{2}()-.V_{2}(\theta)\right) /\left(V_{1}()-.V_{1}(\theta)\right)$. Our result is a special case of an additively decomposable representation on an infinite product space. General results on this topic are in Streufert (1995) and Wakker and Zank (1999).

Proof of Observation 2.2. Monotonicity follows from the $D U$ representation. For ultimately constant programs, constant-equivalence was not used in the proof of Theorem 2.1, and tail-robustness was used only to show that $\rho>1$ cannot be. The latter is also excluded by monotonicity, as indicated in the proof of Theorem 2.1. To show that tail robustness can be replaced by monotonicity for bounded programs, assume that $\mu \succcurlyeq x_{t} \succcurlyeq \nu$ for all $t$, and that $\alpha$ is the constant-equivalent of $x$. Then, by monotonicity, $x_{T} \mu \succcurlyeq x \sim \alpha \succcurlyeq x_{T} \nu$ for all $T$. By $D U$ for ultimately constant programs, $D U\left(x_{T} \mu\right) \geq D U(\alpha) \geq D U\left(x_{T} \nu\right)$ for all $T$. Because $D U\left(x_{T} \mu\right)$ and $D U\left(x_{T} \nu\right)$ converge to each other, they converge to $D U(\alpha)$. They also converge to $D U(x)$, which, hence, is equal to $D U(\alpha)$ and evaluates $x$. 


\subsection{Appendix B. Example}

For general domains with all programs incorporated, it is not very clear how preference relations can be constructed that satisfy all the axioms of Koopmans (1960). The following example illustrates for a natural case that the axioms of Koopmans, also without his axioms P5 or P5', imply that utility must be bounded.

Example 2.4 Assume that $X=\mathbb{R}_{+}$, the set of nonnegative monetary outcomes. Assume, as in Koopmans (1960), that $\succcurlyeq$ is defined on the whole set of programs $\Pi_{i=1}^{\infty} X$, and that there exists a function $U: \Pi_{i=1}^{\infty} X \rightarrow \mathbb{R}$ that evalutes $\succcurlyeq$, i.e. a consumption program is preferred if and only if it has the higher $U$ value, where further $U$ satisfies uniform continuity on equivalence classes with respect to the supnorm. That is, for each program $x$ and each $\epsilon>0$ there exists $\delta>0$ such that $|U(y)-U(x)|<\epsilon$ as soon as the supnorm distance of $y$ to the equivalence-class of $x$ is less than $\delta$ (his Postulate 1). Koopmans also assumed that the range of $U$ is an interval $I_{U}\left(3^{*}\right.$ in Section 3$)$, using this assumption heavily throughout his analysis. We further assume that discounted utility applies to the set $F$ of all programs for which discounted utility is finite, with $u$ continuous and strictly increasing. $F^{c}$, the complement of $F$, contains all programs with infinite discounted utility. Because for every program $z \in F^{c}$ and $x \in F$ we can make a program $y \in F$ with $z$ dominating $y$ in every period and $y \sim x$, by monotonicity and transitivity every program in $F^{c}$ must be strictly preferred to every one in $F$. Hence, $U\left(F^{c}\right)$ exceeds $U(F)$. We conclude that $U(F)$ and $U\left(F^{c}\right)$ partition the interval $I_{U}$ where $U(F)$ comprises the lower part and $U\left(F^{c}\right)$ the upper part.

Take $x \in F$. Then we can strictly improve $x_{1}$, leading to a strictly better program. Apparently $U(F)$ does not contain its upper bound. Next take $x \in F^{c}$. There must be a $t$ with $x_{t}>0$. Then we can strictly lower $x_{t}$ some, leading to a strictly worse program. Apparently $U\left(F^{c}\right)$ does not contain its lower bound. By connectedness, either $U(F)$ or $U\left(F^{c}\right)$ is empty, so that either $F$ or $F^{c}$ is empty. $F$ contains all constant programs and, therefore, $F^{c}$ is empty. This implies that $u$ must be bounded. If $u$ were unbounded, we could construct $x$ with $u\left(x_{t}\right)>1 / \rho^{t-1}$ in $F^{c}$ so that $x$ has infinite discounted utility, contradicting emptiness of $F^{c}$.

Section $8^{*}$ of Koopmans, Diamond, and Williamson (1964) suggested that arcconnectedness together with a "finite interior diameter" of $X$ implies boundedness of utility. The above example suggests that connectedness alone already is irreconcilable with unbounded utility.

The status of Koopmans' boundedness condition (Koopmans, 1960, Postulate P5; Koopmans, 1972, Postulate P5') is not clear. Possibly he had in mind that all 
programs preferred more than a prespecified best program, and programs preferred less than a prespecified worst program, be dropped from the domain, and that all other programs be retained. Such an approach does not work well because then additive representation theorems of Debreu (1960) or Gorman (1968), used in the proofs, can no longer be applied. About the latter point there have been many misunderstandings in the literature (Wakker, 1993b). The only way to have a best and worst program, and at the same time have the full product structures required for the theorems of Debreu (1960) and Gorman (1968), is to have a best and worst consumption, and let the best and worst programs correspond with these consumptions in all periods. Then all pograms can be included, and the domain contains enough subspaces isomorphic to full product spaces to apply known proof techniques. Utility then has to be bounded automatically, which explains claims about Koopmans' analysis with P5/P5' assumed in the main text.

Koopmans (1972, Section $6^{*}$ ) briefly discussed the extension of his results to unbounded progams with finite discounted utility. There are however, apparently, typos in his text, to the extent that we are unable to guess what he may have had in mind. 


\section{The Hyperbolic FACTOR:}

\section{A Measure of Decreasing Impatience}

Many studies have found that discounting is hyperbolic rather than constant. Hyperbolic discounting is becoming increasingly popular in economic applications. Most studies that provide evidence in favor of hyperbolic discounting either are merely qualitative or they depend on assumptions about, or parametric fittings of, utility functions. This chapter ${ }^{1}$ provides a quantitative measure for the degree of deviation from stationarity that can overcome the problems mentioned. This measure, the hyperbolic factor, can easily be calculated from data and does not require knowledge of the utility function. Moreover, it provides simple preference foundations of the most popular discount functions. Thus, the hyperbolic factor provides an easy tool for theoretical preference foundations, critical empirical tests, and quantitative measurements of hyperbolic discounting.

\subsection{Introduction}

Since Samuelson's (1937) introduction of constant discounted utility, this model has been widely accepted as a normative and descriptive model of intertemporal choice. According to general, possibly non-constant, discounted utility, a stream of outcomes is evaluated by first determining the utility of every outcome, i.e. the value of the outcome would it have been received immediately, and then multiplying each utility by a discount factor that corresponds to the time-point of receipt. Constant discounting implies that a preference between two streams of outcomes is not affected if all outcomes in both streams are delayed by the same time interval.

Recently, there has been an increasing number of empirical studies suggesting that discounting is not constant, including Benzion, Rapoport, and Yagil (1989), Bleichrodt and Johannesson (2001), Cairns and van der Pol (2000), Green, Fristoe,

\footnotetext{
${ }^{1}$ This chapter is based on Rohde (2005).
} 
and Myerson (1994), Kirby and Maraković (1995), Mazur (1987, 2001), Read and Read (2004), Rodriguez and Logue (1988), and Thaler (1981). If an early reward and another, later and larger reward are perceived as being equivalent, then delaying both rewards equally will, for most people and animals, result in a strict preference for the later and larger reward, revealing decreasing impatience.

As a consequence of decreasing impatience, individuals' preferences can be dynamically inconsistent. Consider a person who prefers to receive two apples in one year plus one day rather than one apple in one year, but prefers to receive one apple today rather than two apples tomorrow (Thaler, 1981). This person is decreasingly impatient. If his preferences between 'today' and 'tomorrow' remain the same for one year, then in one year from now, he will prefer to receive one apple on that day rather than two apples one day later. Thus, his preferences between the two options will have changed over time. In this sense decreasing impatience may be viewed as reflecting an irrationality.

An increasingly popular model that captures decreasing impatience is hyperbolic discounting (Loewenstein and Prelec, 1992; Harvey, 1986, 1995; Mazur, 1987). This model has been used in many fields (Akerlof, 2002; Harris and Laibson, 2001; Krusell and Smith, 2003; Laibson, 1997; Luttmer and Mariotti, 2003; O'Donoghue and Rabin, 1999; Thaler and Benartzi, 2004). Most studies that provide empirical evidence in favor of hyperbolic discounting assume a particular, often linear, utility function or first need to parametrically fit utility. Thus, the quantitative evidence in favor of hyperbolic discounting is confounded by assumptions about and parametric fittings of utility. Most qualitative studies in favor of hyperbolic discounting only reject constant discounting and provide evidence in favor of general decreasing impatience, not of hyperbolic discounting in particular.

This chapter proposes a simple method to quantify the degree of deviation from stationarity that does not need assumptions about or estimations of utility. That is, a measure of decreasing impatience is introduced, the hyperbolic factor, which can easily be calculated from data without knowledge of utility.

One approach to construct a measure of decreasing impatience would be to find out how impatience changes over time. This would require knowledge of impatience at each time-point, i.e. knowledge of the discount function. Then, to determine this discount function, we would also need to know the utility function. It is, indeed, commonly believed in the field that such a procedure should be followed. Surprisingly, as this paper shows, we do not need to go through all these steps. In fact, measuring the degree of decreasing impatience is even easier than measuring the discount function.

Our method of measurement is similar to the utility measurement method of 
Wakker and Deneffe (1996). There, choices between gambles under uncertainty are constructed in such a manner that subjective probabilities or decision weights cancel from the equations, so that utilities can be measured without the need to measure subjective or weighted probabilities. Similarly, this paper constructs choices between delayed outcomes in such a manner that the absolute level of discounting and the utilities of outcomes cancel from the equations, so that we can measure variations in impatience and, thus, degrees of irrationality, without knowledge of utility or the absolute level of discounting.

As will be shown, the hyperbolic factor is a useful tool in the axiomatization of the various discount functions that exist today. A constant positive hyperbolic factor corresponds to generalized hyperbolic discounting (Loewenstein and Prelec, 1992). Quasi-hyperbolic discounting holds if and only if the hyperbolic factor is equal to zero for all points in time except the present (Phelps and Pollak, 1968). If, in addition, the hyperbolic factor is zero in the present, then constant discounting holds. Thus, estimating the hyperbolic factor and testing whether it is constant will be useful in testing which of the currently used models fit empirical data best and in testing whether these existing models are appropriate at all or whether different models need to be developed.

Prelec (2004) introduced another measure of decreasing impatience. Relative to the hyperbolic factor, his measure is more complicated: it uses the second derivative of the logarithm of the discount function, which can only be obtained after complex measurements of discounting and utility. Moreover, his measure is not constant under hyperbolic discounting. The difference between Prelec's measure and the hyperbolic factor is analogous to the difference between the measure of absolute and that of relative risk aversion (Mas-Colell, Whinston, and Green, 1995). While the former is most useful for CARA-utility (constant absolute risk aversion), the latter is most useful for CRRA-utility (constant relative risk aversion). Similarly, while the hyperbolic factor is most useful for hyperbolic discounting, Prelec's measure will be more useful for other types of discount functions. Finally, the hyperbolic factor is model-free, i.e. it can also be used as a measure of decreasing impatience when preferences cannot be represented by discounted utility. This is not the case for Prelec's measure, which essentially needs a discount function.

Section 3.2 defines the hyperbolic factor. This factor is applied to discounted utility in Section 3.3. All proofs are in the Appendix. 


\subsection{The Hyperbolic Factor Defined}

Let $\mathcal{X}=\mathbb{R}^{m}$ be a set of outcomes ${ }^{2}$ and $\mathcal{T}=\mathbb{R}_{+}$a set of time-points. A timed outcome $(t, \mu)$ yields outcome $\mu$ at time $t$ and nothing $(=0)$ at all other points in time, where $t=0$ corresponds to 'today'. We examine preferences $\succcurlyeq$ over timed outcomes. The relations $\preccurlyeq, \succ, \prec, \sim$ are as usual. Preferences over outcomes are derived from preferences over timed outcomes consumed today, i.e. $\chi \succcurlyeq \mu$ if and only if $(0, \chi) \succcurlyeq(0, \mu)$.

We assume that $\succcurlyeq$ is a weak order, i.e $\succcurlyeq$ is complete $((s, \mu) \succcurlyeq(t, \chi)$ or $(t, \chi) \succcurlyeq(s, \mu)$ for all $\mu, \chi \in \mathcal{X}$ and $s, t \in \mathcal{T}$, possibly both) and transitive. Preferences are monotonic if $\chi \succcurlyeq \mu$ implies $(t, \chi) \succcurlyeq(t, \mu)$ for every $t \in \mathcal{T}$, and $\chi \succ \mu$ implies $(t, \chi) \succ(t, \mu)$ for every $t \in \mathcal{T}$. Preferences are impatient if for every $s<t, \chi \succ 0$ implies $(s, \chi) \succ(t, \chi)$ and $\chi \prec 0$ implies $(s, \chi) \prec(t, \chi)$. Preferences are continuous if for every $(t, \chi)$ the sets $\{(s, \mu) \in \mathcal{T} \times \mathcal{X} \mid(s, \mu) \succcurlyeq(t, \chi)\}$ and $\{(s, \mu) \in \mathcal{T} \times \mathcal{X} \mid(s, \mu) \preccurlyeq(t, \chi)\}$ are closed. Throughout this paper we assume that preferences constitute a continuous, monotonic, and impatient weak order.

Consider two equivalent timed outcomes $(s, \mu) \sim(t, \chi)$, with $s<t$ and $\mu \nsim \chi$. Then we have either $\chi \succ \mu \succ 0$ or $\chi \prec \mu \prec 0$ ( $\mu$ is 'moderate' and $\chi$ is 'extreme'). If the outcome $\mu$ is delayed by an interval $\tau$, then stationarity implies that the outcome $\chi$ should also be delayed by $\tau$ in order to maintain indifference. Thus, under stationarity $(s, \mu) \sim(t, \chi)$ implies $(s+\tau, \mu) \sim(t+\tau, \chi)$. Stationarity reflects constant impatience.

The preference relation $\succcurlyeq$ exhibits decreasing impatience if for all $s<t, \tau \in \mathcal{T}$, (i) $\chi \succ \mu \succ 0$ and $(s, \mu) \sim(t, \chi)$ imply $(t+\tau, \chi) \succcurlyeq(s+\tau, \mu)$, and (ii) $\chi \prec \mu \prec 0$ and $(s, \mu) \sim(t, \chi)$ imply $(t+\tau, \chi) \preccurlyeq(s+\tau, \mu)$; increasing impatience holds if the implied preferences are always the reverse. Thus, with decreasing impatience, when we consider two equivalent timed outcomes, then delaying both outcomes equally will result in less distinction between the time-points, and, thus, more preference for the timed outcome with the preferred outcome. In this sense, decreasing impatience reflects that a time difference becomes decreasingly important as it lies farther in the future.

Assume another preference relation $\succcurlyeq^{*}$, which also is a continuous, monotonic, and impatient weak order. Preferences $\succcurlyeq^{*}$ exhibit more decreasing impatience than $\succcurlyeq$ if for all $s<t, \tau, \sigma \in \mathcal{T}$ and $\mu \nsim \chi$, (i) $\chi^{*} \succ^{*} \mu^{*} \succ^{*} 0,(s, \mu) \sim(t, \chi)$, $(s+\sigma, \mu) \sim(t+\tau, \chi)$, and $\left(s, \mu^{*}\right) \sim^{*}\left(t, \chi^{*}\right)$ imply $\left(t+\tau, \chi^{*}\right) \succcurlyeq^{*}\left(s+\sigma, \mu^{*}\right)$, and

\footnotetext{
${ }^{2}$ All results in this paper remain valid if $\mathcal{X}$ is a connected topological space containing a reference outcome 'nothing.' $\mathcal{X}$ can, for instance, be any convex subset of $\mathbb{R}^{m}$ containing zero, or a set of non-quantified health states.
} 
(ii) $\chi^{*} \prec^{*} \mu^{*} \prec^{*} 0,(s, \mu) \sim(t, \chi),(s+\sigma, \mu) \sim(t+\tau, \chi)$, and $\left(s, \mu^{*}\right) \sim^{*}\left(t, \chi^{*}\right)$ imply $\left(t+\tau, \chi^{*}\right) \preccurlyeq^{*}\left(s+\sigma, \mu^{*}\right)$ (Prelec, 2004).

Consider again two equivalent timed outcomes $(s, \mu) \sim(t, \chi)$ with $s<t$ and $\mu \nsim \chi$. Assume that $(s+\sigma, \mu) \sim(t+\tau, \chi)$. Decreasing (increasing) impatience implies that $\tau-\sigma>0(\tau-\sigma<0)$. An obvious measure of decreasing impatience is, therefore, $\tau-\sigma$. This measure $\tau-\sigma$, however, will depend on $s, t, \sigma, \mu$, and $\chi$, and will be hard to compare across different outcomes and time-points. The main purpose of this paper is to propose a transformation of this measure that is better suited as a measure of impatience, and that can be compared more easily across different outcomes and time-points. This proposed measure, the hyperbolic factor, is defined next. It is just as easily observable from preferences as $\tau-\sigma$ itself. Unlike $\tau-\sigma$, however, it will be constant, i.e. independent of $s, t, \sigma, \mu$ and $\chi$, for all hyperbolic discounting models currently used in the literature, as we will see in Section 3.3. Outcomes $\mu, \chi \in \mathcal{X}$ and time-points $s, t, \sigma, \tau \in \mathcal{T}$, with $s<t$, $\tau>0$, form an indifference pair if

$$
(s, \mu) \sim(t, \chi) \text { and }(s+\sigma, \mu) \sim(t+\tau, \chi) .
$$

\section{Definition 3.1}

For every indifference pair as in eq. 3.1 the hyperbolic factor is defined as

$$
\frac{\tau-\sigma}{t \sigma-s \tau}
$$

For general preferences, a hyperbolic factor may not always be defined for every outcome $\chi$ and all $s<t$ and $\tau>0$. For instance, there may be no $\mu$ and $\sigma$ that satisfy eq. 3.1. Our assumptions about preferences imply that such a case can never arise, so that a $\mu$ and $\sigma$ as described can always be found. This claim is formalized in the next theorem.

Theorem 3.2 For every $\chi \nsim 0, s<t$, and $\tau>0$, there are a unique $\sigma$ and $a \mu$, such that an indifference pair as in eq. 3.1 results.

Now we can define the function $H$ for every $\chi \nsim 0, s<t$, and $\tau>0$, as

$$
H(s, t, \chi, \tau)=\frac{\tau-\sigma}{t \sigma-s \tau}
$$

where $\sigma$ is such that together with a $\mu$ and the arguments of $H$, it yields an indifference pair as in eq. 3.1. The function $H$ gives the hyperbolic factors. In general, $H$ need not always be regular, i.e. $H$ is infinite if $t \sigma=s \tau$, and negative in spite of strongly decreasing impatience if $t \sigma<s \tau$. Yet, as we will see later, for all 
discounted utility models popular in the literature, regularity holds, i.e. for every indifference pair as in eq. 3.1 we have $t \sigma>s \tau$.

Note that for every indifference pair, a hyperbolic factor can be calculated. From $n$ indifferences as in eq. 3.1 with varying time-points, $\left(\begin{array}{l}n \\ 2\end{array}\right) / 2$ hyperbolic factors can be calculated. Non-negative hyperbolic factors correspond to decreasing impatience. We will see in Section 3.3 that hyperbolic discounting induces nonnegative hyperbolic factors, and, thus, decreasing impatience.

Theorem 3.3 Let regularity hold. Preferences $\succcurlyeq$ exhibit decreasing impatience if and only if $H \geq 0$. Preferences $\succcurlyeq$ exhibit increasing impatience if and only if $H \leq 0$.

The hyperbolic factor also serves as a measure of decreasing impatience, as shown in the next theorem. Thus, it properly captures Prelec's (2004) relative decreasing impatience. When we consider another preference relation $\succcurlyeq^{*}$, then it is assumed that $\succcurlyeq^{*}$ is a continuous, monotonic and impatient weak order, and that the corresponding hyperbolic factors are given by $H^{*}(s, t, \chi, \tau)$.

Theorem 3.4 Let regularity hold. Preferences $\succcurlyeq^{*}$ exhibit more decreasing impatience than $\succcurlyeq$ if and only if $H^{*}\left(s, t, \chi^{*}, \tau\right) \geq H(s, t, \chi, \tau)$ for all $s, t, \tau, \chi, \chi^{*}$.

Thus, we have shown that the hyperbolic factor is an appropriate model-free measure of decreasing impatience that can easily be obtained from an indifference pair.

\subsection{The Hyperbolic Factor and Discounted Uti- lity}

Discounted utility holds if there exist a discount function $\phi$ and a utility function $u$ such that preferences $\succcurlyeq$ can be represented by

$$
D U(t, \mu)=\phi(t) u(\mu),
$$

where $\phi$ is continuous and strictly decreasing, $\phi(0)=1, \phi(t)>0$ for every $t$, and $u$ is continuous, $u(0)=0$, and there is an outcome $\chi \in \mathcal{X}$ with $u(\chi) \neq 0$. Fishburn and Rubinstein (1982) characterized discounted utility. In this section we will assume that discounted utility holds. Thus, preferences still constitute a continuous, monotonic and impatient weak order as in Section 3.2. We will not assume regularity, but instead derive it later from other assumptions. 
Under discounted utility, the hyperbolic factor is independent of the outcomes, as the following theorem shows.

Theorem 3.5 Let discounted utility hold. Then $H(s, t, \chi, \tau)$ is independent of $\chi$.

Two decision-makers with different discount functions $\phi$ and $\phi^{*}$ that are related by a power transformation $\phi^{*}(t)=[\phi(t)]^{c}$ have equal hyperbolic factors, as stated in Observation 3.6. Thus, in order to measure deviations from stationarity, we do not even need to know how much people discount in an absolute sense. This observation underlies the possibility to analyze decreasing impatience without a need to measure the discount function or utility.

Observation 3.6 Let discounted utility hold. Consider two discount functions $\phi$ and $\phi^{*}$ with corresponding $H$ and $H^{*}$. If there is a $c \in \mathbb{R}$ such that $\phi^{*}(t)=[\phi(t)]^{c}$ then $H^{*}\left(s, t, \chi^{*}, \tau\right)=H(s, t, \chi, \tau)$ for every $s, t, \tau, \chi, \chi^{*}$.

\subsubsection{Constant Discounting}

Constant discounting has been a traditional assumption in economics. Preferences satisfy constant discounting if there is a constant discount factor $\delta$ such that $\phi(t)=\delta^{t}$ for every $t$. Constant discounting is equivalent to the hyperbolic factor always being zero.

Theorem 3.7 The following two statements are equivalent under discounted utility.

(i) Preferences $\succcurlyeq$ satisfy constant discounting.

(ii) $H(s, t, \chi, \tau)=0$ for all $s, t, \chi, \tau$.

\subsubsection{Generalized Hyperbolic Discounting}

Following up on the empirical studies that found violations of stationarity, Loewenstein and Prelec (1992) introduced the generalized hyperbolic discount function, which is defined by

$$
\phi(t)=(1+h t)^{-r / h},
$$

with $h>0, r>0$. Generalized hyperbolic discounting is equivalent to the hyperbolic factor being a positive constant.

Theorem 3.8 The following two statements are equivalent under discounted utility. 
(i) Preferences $\succcurlyeq$ satisfy generalized hyperbolic discounting $\phi(t)=(1+h t)^{-r / h}$ with parameter $h>0$.

(ii) There is a constant $h>0$ such that $H(s, t, \chi, \tau)=h$ for all $s, t, \chi, \tau$.

Mazur (1987) tested a necessary condition for hyperbolic discounting that also did not require knowledge of utility. We provide a testable condition that is not only necessary, but also sufficient for hyperbolic discounting, as Theorem 3.8 shows.

\subsubsection{Harvey Discounting}

Harvey (1986) proposed a discount function given by

$$
\phi(t)=(1+t)^{-r} .
$$

This Harvey discounting is equivalent to generalized hyperbolic discounting with a hyperbolic factor that equals one.

Theorem 3.9 The following two statements are equivalent under discounted utility.

(i) Preferences $\succcurlyeq$ satisfy Harvey discounting.

(ii) $H(s, t, \chi, \tau)=1$ for all $s, t, \chi, \tau$.

\subsubsection{Proportional Discounting}

Mazur (1987) and Harvey (1995) proposed a discount function given by

$$
\phi(t)=(1+h t)^{-1} .
$$

This proportional discounting is equivalent to generalized hyperbolic discounting with $r=h$.

It follows that the hyperbolic factor does not distinguish between the generalized hyperbolic discounting of Loewenstein and Prelec (1992) and the proportional discounting of Mazur (1987) and Harvey (1995). This is because the hyperbolic factor only restricts the parameter $h$ and not the parameter $r$ as we saw in Observation 3.6. Indeed, on our domain of timed outcomes the two models cannot be distinguished, because they differ only regarding the absolute level of discounting and not regarding the degree of decreasing impatience. This finding reflects once more that changes in impatience and the corresponding irrationalities can be investigated independently of the absolute level of discounting. 


\subsubsection{Quasi-hyperbolic Discounting}

Phelps and Pollak (1968) introduced quasi-hyperbolic discounting, as used by Laibson (1997) and many others. The quasi-hyperbolic discount function is given by

$$
\phi(t)= \begin{cases}1 & \text { if } t=0 \\ \beta \delta^{t} & \text { if } t>0\end{cases}
$$

for some $\beta \leq 1$, and some $\delta>0$.

Quasi-hyperbolic discounting is equivalent to stationarity for all of the future except the present.

Theorem 3.10 The following two statements are equivalent under discounted utility.

(i) Preferences $\succcurlyeq$ satisfy quasi-hyperbolic discounting.

(ii) $H(s, t, \chi, \tau)=0$ for all $s>0, t, \chi, \tau$.

Thus, when combined with Fishburn and Rubinstein's (1982) preference foundation of discounted utility, this section provided preference foundations for all currently popular discount models.

\subsection{Conclusion}

This paper has introduced the hyperbolic factor, a quantitative measure of decreasing impatience, which can easily be obtained from an indifference pair. In addition to being a simple measure, the hyperbolic factor is useful in characterizing all popular discount models. Hyperbolic discounting holds if and only if the hyperbolic factor is constant and positive. The discount function of Harvey (not proportional discounting) applies if and only if the hyperbolic factor is always equal to one. Quasi-hyperbolic discounting holds if and only if the hyperbolic factor is equal to zero for all future points in time except the present. If, in addition, the hyperbolic factor is equal to zero today, then constant discounting holds.

A direction for future research is to calculate hyperbolic factors from data, which will illustrate how strong the evidence in favor of hyperbolic discounting is. A major advantage of such future studies, in comparison to earlier ones, is that they will not be confounded by assumptions about or estimations of instant utility functions. Testing whether hyperbolic factors are constant and positive will indicate whether hyperbolic discounting is the appropriate alternative to constant discounting. 


\subsection{Appendix}

Proof of Theorem 3.2

Consider $s, t, \chi, \tau$ with $0 \leq s<t, \chi \nsim 0$, and $\tau>0$. Assume that $\chi \succ 0$. By monotonicity and impatience we know that ${ }^{3}$

$$
(s, 0) \sim(t, 0) \prec(t, \chi) \prec(s, \chi) .
$$

By continuity of preferences and connectedness of $\mathbb{R}^{m}$ there must then be a $\mu$ with $(s, \mu) \sim(t, \chi)$ and $\chi \succ \mu \succ 0$. Thus,

$$
(s, \mu) \sim(t, \chi) \succ(t+\tau, \chi) \succ(t+\tau, \mu) .
$$

By continuity there must then be a $\sigma$ with $(s+\sigma, \mu) \sim(t+\tau, \chi)$. By replacing all ' $\succ$ ' by ' $\prec$ ' and all ' $\prec$ ' by ' $\succ$ ', this reasoning shows that similar things hold for $\chi \prec 0$.

By monotonicity and impatience, $\mu$ is unique up to indifference and $\sigma$ is unique.

\section{Proof of Theorem 3.3}

By regularity, we have $H \geq 0$ if and only if $\tau-\sigma \geq 0$ for all indifference pairs as in eq. 3.1. Thus, by Theorem 3.2, we have $H \geq 0$ if and only if decreasing impatience holds.

Proof of Theorem 3.4

By regularity, we have $H^{*}\left(s, t, \chi^{*}, \tau\right) \geq H(s, t, \chi, \tau)$ for every $s, t, \tau, \chi, \chi^{*}$ if and only if $\sigma^{*} \leq \sigma$ for all $s<t, \chi \nsim 0, \tau>0$ with $(s, \mu) \sim(t, \chi),(s+\sigma, \mu) \sim(t+\tau, \chi)$ and $\left(s, \mu^{*}\right) \sim^{*}\left(t, \chi^{*}\right),\left(s+\sigma^{*}, \mu^{*}\right) \sim^{*}\left(t+\tau, \chi^{*}\right)$, which, by impatience, holds if and only if $\succcurlyeq^{*}$ exhibits more decreasing impatience than $\succcurlyeq$.

\section{Proof of Theorem 3.5}

Let $H(s, t, \chi, \tau)=h$ and $H\left(s, t, \chi^{*}, \tau\right)=h^{*}$. Then there are $\mu, \sigma, \mu^{*}, \sigma^{*}$, with $(s, \mu) \sim(t, \chi),(s+\sigma, \mu) \sim(t+\tau, \chi),\left(s, \mu^{*}\right) \sim\left(t, \chi^{*}\right)$ and $\left(s+\sigma^{*}, \mu^{*}\right) \sim\left(t+\tau, \chi^{*}\right)$. By discounted utility it follows that

$$
\phi(s) u(\mu)=\phi(t) u(\chi)
$$

\footnotetext{
${ }^{3}$ By the definition of a timed outcome it follows that $(s, 0) \sim(t, 0)$ for every $s, t$.
} 
and

$$
\phi(s+\sigma) u(\mu)=\phi(t+\tau) u(\chi) .
$$

Therefore,

$$
\frac{u(\mu)}{u(\chi)}=\frac{\phi(t)}{\phi(s)}=\frac{\phi(t+\tau)}{\phi(s+\sigma)}
$$

Similarly,

$$
\frac{u\left(\mu^{*}\right)}{u\left(\chi^{*}\right)}=\frac{\phi(t)}{\phi(s)}=\frac{\phi(t+\tau)}{\phi\left(s+\sigma^{*}\right)} .
$$

By impatience it then follows that $\sigma^{*}=\sigma$ and $h=h^{*}$. This proves our result.

A similar reasoning proves Observation 3.6.

Proof of Theorem 3.7

Let $H(s, t, \chi, \tau)=0$ for all $s, t, \chi, \tau$. Then

$$
(s, \mu) \sim(t, \chi)
$$

if and only if

$$
(s+\tau, \mu) \sim(t+\tau, \chi),
$$

i.e. stationarity holds. Thus, for every $s, t, \sigma \in \mathcal{T}$,

$$
\frac{\phi(s)}{\phi(t)}=\frac{\phi(s+\tau)}{\phi(t+\tau)} .
$$

Therefore, by setting $s=0$, for every $t, \tau \in \mathcal{T}$,

$$
\phi(t) \phi(\tau)=\phi(t+\tau) .
$$

By Cauchy's functional equation it follows that there must be a $c \in \mathbb{R}$ such that $\phi(t)=e^{c t}$ for every $t \in \mathcal{T}$. Now let $\delta=e^{c}$. Then, $\phi(t)=\delta^{t}$. The converse follows easily.

\section{Proof of Theorem 3.8}

Let there be a constant $h>0$ such that $H(s, t, \chi, \tau)=h$ for all $s, t, \chi, \tau$. Assume that

$$
u(\mu)=\phi(t) u(\chi) \text { and } \phi(\sigma) u(\mu)=\phi(t+\tau) u(\chi),
$$

with $t>0$. Then we must have

$$
\frac{\tau-\sigma}{t \sigma}=h .
$$


Let $k=1+h t$. It follows that $t+\tau=t+k \sigma$. Moreover, $k$ is a constant that depends only on $t$. Loewenstein and Prelec (1992) showed that this implies that the discount function is of the generalized hyperbolic form. Thus, there must be parameters $\tilde{h}, r$ such that $\phi(t)=(1+\tilde{h} t)^{-r / \tilde{h}}$. It follows that $\tilde{h}=h$. From the assumption that $\phi(\cdot)$ is strictly decreasing it follows that $r>0$. This proves one direction of our result. The proof of the other direction is straightforward.

\section{Proof of Theorem 3.9}

Harvey discounting is equivalent to generalized hyperbolic discounting with $h=1$.

Proof OF TheOREM 3.10

Let $H(s, t, \chi, \tau)=0$ for all $s>0, t, \chi, \tau$. Then for every $s, t, \sigma \in \mathcal{T}$, with $s, t>0$

$$
\frac{\phi(s)}{\phi(t)}=\frac{\phi(s+\sigma)}{\phi(t+\sigma)} \text {. }
$$

Let $s_{0}>0$. Define the function $\phi_{0}$ on $\mathcal{T}$ by $\phi_{0}(t)=\phi\left(t+s_{0}\right) / \phi\left(s_{0}\right)$. Then for every $s, t, \sigma \in \mathcal{T}$,

$$
\frac{\phi_{0}(s)}{\phi_{0}(t)}=\frac{\phi\left(s+s_{0}\right)}{\phi\left(t+s_{0}\right)}=\frac{\phi\left(s+s_{0}+\sigma\right)}{\phi\left(t+s_{0}+\sigma\right)}=\frac{\phi_{0}(s+\sigma)}{\phi_{0}(t+\sigma)} .
$$

Moreover, $\phi_{0}(0)=1$. By Cauchy's functional equation it follows that there must be a $c_{0} \in \mathbb{R}$ such that $\phi_{0}(t)=e^{c_{0} t}$ for every $t \in \mathcal{T}$. Therefore, $\phi\left(t+s_{0}\right)=e^{c_{0} t} \phi\left(s_{0}\right)$ for every $t \in \mathcal{T}$. Thus, $\phi(t)=e^{c_{0}\left(t-s_{0}\right)} \phi\left(s_{0}\right)$ for all $t \geq s_{0}$. Define $\delta_{0}=e^{c_{0}}$ and $\beta_{0}=e^{-c_{0} s_{0}} \phi\left(s_{0}\right)$. Then $\phi(t)=\beta_{0} \delta_{0}^{t}$ for all $t \geq s_{0}$. Similarly, consider an $s_{1}$ with $0<s_{1}<s_{0}$ and with corresponding $\beta_{1}$ and $\phi_{1}$. It follows that for all $t \geq s_{0}$, $\phi(t)=\beta_{1} \delta_{1}^{t}=\beta_{0} \delta_{0}^{t}$, so $\beta_{0}=\beta_{1}$ and $\delta_{0}=\delta_{1}$. We can continue this argument repeatedly.

Thus, letting $\beta=\beta_{0}$ and $\delta=\delta_{0}$ we obtain $\phi(t)=\beta \delta^{t}$ for all $t>0$. By definition we have $\phi(0)=1$. 
Time-Tradeoff Sequences for Quantifying and Visualising the Degree of Time Inconsistency, Using ONLY PENCIL AND PAPER

This chapter ${ }^{1}$ introduces time-tradeoff (TTO) sequences as a new tool for analyzing intertemporal preferences. TTO sequences yield a new way to measure temporal discounting, while minimizing distortions due to violations of intertemporal separability. They make it particularly easy to observe and exactly quantify deviations from stationarity and the implied proneness to choice anomalies. TTO sequences can easily be administered and analyzed, using only pencil and paper, and do not need any assumption about utility, or estimation thereof. They allow for the empirical discrimination between several hyperbolic discounting models that have been proposed in the literature as alternatives to constant discounting, such as quasi-hyperbolic, proportional, and generalized hyperbolic discounting. We tested the feasibility of TTO sequences in an experiment. Our findings suggest some new directions for theories of intertemporal choice.

\subsection{Introduction}

Many decisions involve tradeoffs over time. The most popular model for evaluating streams of outcomes over time, also assumed in this paper, is (general) discounted utility. In this model, streams of outcomes are evaluated by summing the discounted utilities of the outcomes received at various timepoints. In traditional approaches, the measurement of the discount function is difficult because both this function and the utility function are unknown parameters that have to be

\footnotetext{
${ }^{1}$ This chapter is based on Attema, Bleichrodt, Rohde, and Wakker (2006).
} 
measured simultaneously (Coller, Harrison, and Rutström, 2005). The difficulty is aggravated because separability of preferences over disjoint time periods, an assumption of the model, is extensively violated empirically, distorting assessments of discounting. We introduce time-tradeoff (TTO) sequences as a new and simple tool for measuring discount functions. It turns out that we need no measurement of utility, or assumption about utility, to obtain the discount function. Distortions due to violations of time separability are minimized.

Samuelson (1937) introduced constant discounting, where a preference between two outcome streams does not change if all outcomes are delayed by an equal time period, reflecting constant impatience, a property also known as stationarity. Constant discounting has long been the standard for intertemporal choice in economics. An attractive feature is that, under some extra assumptions, constant discounting implies dynamically consistent behavior: plans for future decisions will be adhered to, and no arbitrage is possible.

Empirical evidence has revealed many violations of stationarity. Mostly, impatience is decreasing rather than constant (Frederick, Loewenstein, and O'Donoghue, 2002). People who at present are not willing to wait for an improved but delayed outcome due to impatience, become willing to wait if all outcomes are delayed by the same amount of time. These people, thus, become less impatient as time proceeds. Under common assumptions, decreasing impatience implies dynamic inconsistency, which is usually considered irrational. All kinds of choice anomalies result, such as proneness to arbitrage.

Hyperbolic discounting models have been developed so as to model decreasing impatience. For example, quasi-hyperbolic discounting (Phelps and Pollak, 1968) assumes constant impatience for all future timepoints, but decreasing impatience at present. Then time inconsistency arises only if immediate consumption is involved. Generalized hyperbolic discounting (Loewenstein and Prelec, 1992) allows decreasing impatience at all timepoints. Analyses of traditional economic models change because of these new ways of discounting, and many previously unexplained phenomena can now be accommodated (Laibson, 1997). Hence, hyperbolic discounting is popular today.

Prelec (2004) introduced a theoretical measure of decreasing impatience, being the convexity index $-\frac{\ln (\varphi)^{\prime \prime}}{\ln (\varphi)^{\prime}}$ of the logarithm of the discount function $\varphi .^{2} \mathrm{He}$ demonstrated that this measure identifies different degrees of proneness to inconsistencies and arbitrage when impatience is decreasing. He wrote: "Decreasing impatience provides a natural criterion for assessing whether a set of time pre-

\footnotetext{
${ }^{2}$ The index measures concavity for increasing functions, and convexity for decreasing functions such as $\varphi$.
} 
ferences represents a more or less severe departure from the stationarity axiom. The criterion is associated with a simple normative diagnostic-the selection of inefficient (dominated) outcomes in two-stage decision problems" (p. 526).

At this stage, it may seem to be difficult to elicit or implement Prelec's measure in practice. It, apparently, first requires the measurement of the discount function and, next, taking the logarithm and determining its second derivative over its first. To measure the discount function, we, apparently, have to measure utility, or at least make assumptions about utility, because the discount function determines behavior only in combination with utility. Some analyses in the literature parametrically estimated utility and, subsequently, used these estimates to measure the discount function (Chapman, 1996). Most analyses simply equated outcomes with utility, which amounts to the assumption of linear utility. Such assumptions can confound findings about discounting.

TTO sequences provide a new way of directly measuring the degree of deviation from stationarity and the degree of time-inconsistent behavior. Surprisingly, we can immediately estimate Prelec's index $-\frac{\ln (\varphi)^{\prime \prime}}{\ln (\varphi)^{\prime}}$ of time inconsistency and graphically depict it, using only pencil and paper, without need to carry out the measurements and calculations mentioned above. In particular, we need not determine the utility function. Through TTO sequences we can immediately tell who of two persons satisfies more decreasing impatience, and we can identify groups of people who are especially prone to losses and arbitrage because of time inconsistency, as we show in a representation theorem. TTO sequences are easy to comprehend for subjects, leading to reliable data.

TTO sequences, together with one simple choice between outcome streams with two nonzero outcomes, completely identify the time discount function. Again, for this measurement no assumption about utility is needed. Our method is therefore, obviously, robust against distortions and nonlinearities in utility, and can be applied to general outcome sets, such as finite sets of qualitative health states.

We show how TTO sequences can test which of several hyperbolic models considered in the literature can be applied, and of those that can be, which best fit the choices of individuals. Until now, most studies only rejected constant discounting, but did not test which alternative was better. In an experiment, we demonstrate the feasibility of our method by measuring TTO sequences of 55 subjects.

Our experimental findings lead to a number of suggestions for new models of intertemporal choice. Several recent studies, discussed in Section 4.9, have found increasing impatience, which casts doubt on the universal decreasing impatience commonly assumed in time-preference theories. Our study also finds a majority of increasing, rather than decreasing, impatience for the present and near future. 
After the present and near future, impatience becomes constant.

Most analyses of intertemporal discounting considered in the literature so far have focused entirely on decreasing impatience. The data of our study and some other recent studies suggest that the development of new tools for analyzing increasing impatience will be worthwhile. This point can be compared to the risk field, where tools for analyzing risk seeking are needed also if the majority of subjects exhibit risk aversion. Without such flexibility of modeling, data fitting is not possible at the individual level.

We also find some fundamental violations of the general discounted utility model. This suggests that generalizations, primarily relaxing temporal separability, are desirable.

The outline of this chapter is as follows. Section 4.2 describes discounted utility and the various families of discount functions considered in this chapter. TTO sequences and curves are presented in Section 4.3. Section 4.4 demonstrates theoretically that TTO sequences capture the degree of deviation from stationarity, and the proneness to choice anomalies. Section 4.5 illustrates some applications, and Section 4.6 shows how discount functions can be measured using TTO sequences. Experimental details are in Section 4.7, and results in Section 4.8. Section 4.9 contains a discussion.

\subsection{Discounted Utility}

We consider preferences between outcome streams. An outcome stream $\left(t_{1}\right.$ : $\left.x_{1}, \ldots, t_{m}: x_{m}\right)$ yields outcome $x_{i}$ at timepoint $t_{i}$ for $i=1, \ldots, m$ and nothing at other timepoints. For simplicity of presentation we assume that outcomes are monetary and nonnegative, with "nothing" equated with the 0 outcome. Our measurement method can equally well be applied to other outcomes, with the outcome set for instance a finite set of qualitative health states, but we will not pursue this point. Timepoint $t=0$ corresponds with the present. Under discounted utility (which in this chapter refers to general, possibly nonconstant, discounting), outcome streams are evaluated through

$$
D U\left(t_{1}: x_{1}, \ldots, t_{m}: x_{m}\right)=\sum_{i=1}^{m} \varphi\left(t_{i}\right) U\left(x_{i}\right) \text {, }
$$

where $\varphi$ is the discount function and $U$ the (instant) utility function, with $\varphi(t)>0$ for all $t, \varphi$ strictly decreasing (impatience) and continuous, $U(0)=0$, and $U$ continuous and strictly increasing. $\varphi$ and $U$ are ratio scales, meaning that each 
is unique up to a positive scale factor. Throughout this chapter we assume that discounted utility holds. In the literature, a normalization $\varphi(0)=1$ is sometimes assumed, but it is more convenient for us not to commit to a scaling.

Constant discounting holds if $\varphi(t)=\delta^{t}$ for a discount factor $\delta$ with $0<\delta \leq 1$. Constant discounting has been the traditional assumption. Then a preference between two outcome streams does not change if all outcomes are delayed by an equal amount of time $\varepsilon$, a preference condition known as stationarity or constant impatience. Under such a delay, the discounted utility of both sequences is multiplied by the same constant $\delta^{\varepsilon}$, so that their ordering is not affected. It is well-known that the reversed implication also holds under common assumptions, that is, constant impatience implies constant discounting (Koopmans, 1960).

In psychological studies it has often been found that people have decreasing impatience, i.e. their willingness to wait increases as outcomes are delayed. A popular model that captures decreasing impatience is the quasi-hyperbolic discount model (Phelps and Pollak, 1968), where the discount function is given by

$$
\varphi(t)= \begin{cases}1 & \text { if } t=0 \\ \beta \delta^{t} & \text { if } t>0\end{cases}
$$

for a constant $\beta \leq 1$ with, again, $0<\delta \leq 1$. Under quasi-hyperbolic discounting we have decreasing impatience only at timepoint 0 , and constant impatience thereafter.

A model that captures decreasing impatience not only for the present, but also for future timepoints, is generalized hyperbolic discounting (Loewenstein and Prelec, 1992), defined by

$$
\varphi(t)=(1+h t)^{-r / h},
$$

with $h>0$ and $r>0$. Harvey (1986, Eq. 7) considered the special case $h=1$. Mazur (1987) and Harvey (1995, "proportional discounting") considered the case $h=r$.

In general, violations of stationarity need not imply time inconsistency, contrary to claims sometimes made in the literature, but in agreement with some careful discussions (Dasgupta and Maskin, 2005, section I; Harvey, 1995, p. 389; Thaler, 1981). For example, you may have a special preference for apples on Tuesday, and prefer two apples on Tuesday to one apple on Monday, but not prefer two apples on Wednesday to one apple on Tuesday. This entails a violation of stationarity, but no inconsistency. All the time you consistently have and predict your preferences, and you never change plans. Such discrepancies between stationarity 
and time inconsistency are caused by nonhomogeneity of time. As is common in the literature, we assume homogeneous time henceforth, so that at every timepoint your decisions can be based on stopwatch time, and nonconstant impatience can be equated with time inconsistency. Then nonconstant impatience entails a vulnerability to arbitrage, where a person first pays to obtain an exchange one way and later pays again to reverse the exchange, ending up in the original position less some money. We will come back to this point in Section 4.4.

\subsection{Deriving the Degree of Time Inconsistency from TTO-Sequences}

A time-tradeoff (TTO) sequence is a sequence $t_{0}, \ldots, t_{n}$ of timepoints such that there exist two outcomes $\beta<\gamma$ with

$$
\begin{aligned}
\left(t_{0}: \beta\right) & \sim\left(t_{1}: \gamma\right) \\
\left(t_{1}: \beta\right) & \sim\left(t_{2}: \gamma\right) \\
& \cdot \\
& \cdot \\
& \cdot \\
\left(t_{n-1}: \beta\right) & \sim\left(t_{n}: \gamma\right)
\end{aligned}
$$

That is, each delay between two consecutive timepoints exactly offsets the same improvement of outcome. This delay between two consecutive timepoints, $d_{i}=$ $t_{i}-t_{i-1}$, is called the willingness to wait $(W T W)$. Stationarity means that the WTW is constant. Under decreasing impatience the WTW increases as time proceeds, and under increasing impatience it decreases. Thus, a TTO sequence readily identifies constant, increasing, or decreasing impatience.

TTO sequences are equally spaced in terms of the logarithm of the discount function. Because the derivation of this result may be clarifying, we give it in the main text.

Observation 4.1 For a TTO sequence $t_{0}, \ldots, t_{n}$ :

$$
\ln \left(\varphi\left(t_{0}\right)\right)-\ln \left(\varphi\left(t_{1}\right)\right)=\ln \left(\varphi\left(t_{1}\right)\right)-\ln \left(\varphi\left(t_{2}\right)\right)=\ldots=\ln \left(\varphi\left(t_{n-1}\right)\right)-\ln \left(\varphi\left(t_{n}\right)\right)
$$

\section{Proof}


For a TTO sequence we have

$$
\begin{aligned}
\varphi\left(t_{0}\right) U(\beta) & =\varphi\left(t_{1}\right) U(\gamma), \text { and } \\
\varphi\left(t_{1}\right) U(\beta) & =\varphi\left(t_{2}\right) U(\gamma),
\end{aligned}
$$

implying

$$
\frac{\varphi\left(t_{0}\right)}{\varphi\left(t_{1}\right)}=\frac{U(\gamma)}{U(\beta)}=\frac{\varphi\left(t_{1}\right)}{\varphi\left(t_{2}\right)}=\ldots=\frac{\varphi\left(t_{n-1}\right)}{\varphi\left(t_{n}\right)} .
$$

Here the third and following equalities result from analogous algebraic manipulations. Taking logarithms gives the observation.

The points $t_{0}, \ldots, t_{n}$ are, obviously, also equally spaced in terms of normalizations of $\ln (\varphi(t))$, such as at $t_{0}$ and $t_{n}$. The latter normalization is denoted $\tau$, and is called the time-tradeoff (TTO) curve. It is given by

$$
\tau(t)=\frac{\ln (\varphi(t))-\ln \left(\varphi\left(t_{n}\right)\right)}{\ln \left(\varphi\left(t_{0}\right)\right)-\ln \left(\varphi\left(t_{n}\right)\right)}
$$

Because it is 1 at $t_{0}$ and 0 at $t_{n}$, with $n$ equally big steps of size $1 / n$ in between, we get

$$
\tau\left(t_{j}\right)=1-\frac{j}{n} \text { for all } j
$$

From TTO sequences we can, thus, immediately obtain the graph of the normalized logarithmic discount function. See Figure 4.1 , with points $\left(t_{j}, 1-\frac{j}{n}\right)$ depicted, and linear interpolation. The figure concerns the experiment reported later, and is derived from subject 7's indifferences

$$
\begin{aligned}
(5 \text { months }: € 700) & \sim(7 \text { months }: € 900) \\
(7 \text { months }: € 700) & \sim(9 \text { months }: € 900) \\
(9 \text { months }: € 700) & \sim(12 \text { months }: € 900) \\
(12 \text { months }: € 700) & \sim(18 \text { months }: € 900) \\
(18 \text { months }: € 700) & \sim(24 \text { months }: € 900)
\end{aligned}
$$

so that $n=5, t_{0}=5, t_{1}=7, t_{2}=9, t_{3}=12, t_{4}=18$, and $t_{5}=24$.

The degree of convexity of a function is not affected by normalizations and, hence, the convexity of $\tau$ equals the convexity of $\ln (\varphi)$. As we saw, stationarity, decreasing impatience, and increasing impatience correspond with constant, increasing, and decreasing WTW. Hence, we obtain the following result. 


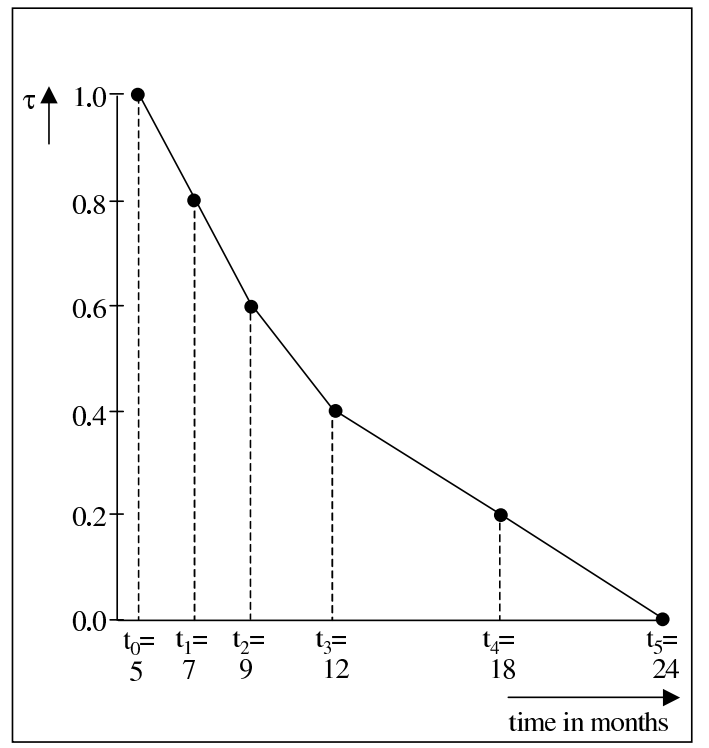

Figure 4.1: The TTO curve $\tau$ of subject 7

Observation 4.2 Stationarity corresponds with linearity of the TTO curve and of $\ln (\varphi)$. Decreasing impatience corresponds with convexity of the TTO curve and of $\ln (\varphi)$. Increasing impatience corresponds with concavity of the TTO curve and of $\ln (\varphi)$. The TTO curve $\tau$ and the logarithm of discounting $\ln (\varphi)$ have the same degree of convexity, i.e.,

$$
-\frac{\tau^{\prime \prime}}{\tau^{\prime}}=-\frac{\ln (\varphi)^{\prime \prime}}{\ln (\varphi)^{\prime}} .
$$

\subsection{TTO-Sequences to Measure Proneness to Ar- bitrage}

In this section, we restrict our attention to simple outcome streams. The following analysis will, contrary to the rest of this chapter, essentially use continuity of utility. A simple outcome stream has at most one nonzero outcome, and can be written as $(s: \alpha)$. Consider the following two indifferences, similar to Eq. 4.2:

$$
\begin{aligned}
& (s: \beta) \sim(t: \gamma) \text { and }(s+\sigma: \beta) \sim(t+\sigma+\rho: \gamma) \\
& \text { for } s<t \text { ( } s \text { for "short" }), \beta<\gamma, \text { and } \sigma>0 .
\end{aligned}
$$


We have $\rho>0$ under decreasing impatience, $\rho=0$ under constant impatience, and $\rho<0$ under increasing impatience. $\rho$ can be taken as an index of deviation from stationarity. Indeed, for $\rho>0$, we have the typical nonstationarity

$$
(s: \beta) \succcurlyeq\left(t^{\prime}: \gamma\right) \text { and }(s+\sigma: \beta) \preccurlyeq\left(t^{\prime}+\sigma: \gamma\right) \text { with one preference strict }
$$

for all $t \leq t^{\prime} \leq t+\rho$ and for no other $t^{\prime}$. The interval $[t, t+\rho]$, thus, indicates a space for arbitrage.

Preference reversals as in Eq. 4.7 are prone to arbitrage. At time 0 the person, when endowed with $(s+\sigma: \beta)$, is willing to exchange it for $\left(t^{\prime}+\sigma: \gamma\right)$. When asked to reconsider at timepoint $\sigma$, the person now perceives of the options as $(s: \beta)$ and $\left(t^{\prime}: \gamma\right)$, and is willing to go back to the $\beta$-option ${ }^{3}$. The person is willing to pay a small amount for at least one of the two exchanges (take it small enough not to affect preference otherwise). Then the person has ended up at the original endowment less some money, which entails arbitrage. Bénabou and Tirole (2002), Gruber and Köszegi (2001), Laibson (1997), O’Donoghue and Rabin (1999), Prelec (2004), Strotz (1956), Thaler and Benartzi (2004), and numerous others derived various choice anomalies from Eq. 4.7, and gave formalizations for these phenomena. For example, a sophisticated person who is informed about the above procedure beforehand may avoid it but then becomes vulnerable to commitments to dominated options, due to lack of future self-control. Other anomalies that can result entail time inconsistency, addiction, and procrastination.

For $\rho<0$ in Eq. 4.6, as typical of increasing impatience, we have

$$
(s: \beta) \preccurlyeq\left(t^{\prime}: \gamma\right) \text { and }(s+\sigma: \beta) \succcurlyeq\left(t^{\prime}+\sigma: \gamma\right) \text { with one preference strict }
$$

for all $t+\rho \leq t^{\prime} \leq t$ and for no other $t^{\prime}$, and $[t+\rho, t]$ indicates a space for arbitrage.

Consider now another preference relation $\succcurlyeq^{*}$, satisfying the assumptions of preceding sections as does $\succcurlyeq$, with corresponding $\varphi^{*}, U^{*}, \tau^{*}$.

Definition $4.3 \succcurlyeq^{*}$ exhibits more decreasing impatience than $\succcurlyeq$ if the equivalences in Eq. 4.6 plus $\left(s: \beta^{*}\right) \sim^{*}\left(t: \gamma^{*}\right)$ imply $\left(s+\sigma: \beta^{*}\right) \preccurlyeq^{*}\left(t+\sigma+\rho: \gamma^{*}\right)$.

Prelec (2004) gave an equivalent definition. Under decreasing impatience for $\succcurlyeq$ and $\succcurlyeq^{*}$, the above condition implies, for

$$
\left(s: \beta^{*}\right) \sim^{*}\left(t: \gamma^{*}\right) \text { and }\left(s+\sigma: \beta^{*}\right) \sim^{*}\left(t+\sigma+\rho^{*}: \gamma^{*}\right),
$$

that either this $\rho^{*}$ exceeds $\rho$, or that such a $\rho^{*}$ does not exist. In the first case the space $\left[t, t+\rho^{*}\right]$ for arbitrage for $\succcurlyeq^{*}$ exceeds the corresponding space $[t, t+\rho]$

\footnotetext{
${ }^{3}$ In the latter step we use homogeneity of time, i.e. the possibility to use stopwatch time, as assumed throughout this chapter.
} 
for $\succcurlyeq$. In the second case the space for arbitrage for $\succcurlyeq^{*}$ is in fact $[t, t+\infty)$, as is readily verified.

There is also interest in increasing impatience, because of which we extend the above definition.

Definition $4.4 \succcurlyeq^{*}$ exhibits more increasing impatience than $\succcurlyeq$ if the equivalences in Eq. 4.6 plus $\left(s: \beta^{*}\right) \sim^{*}\left(t: \gamma^{*}\right)$ imply $\left(s+\sigma: \beta^{*}\right) \succcurlyeq^{*}\left(t+\sigma+\rho: \gamma^{*}\right)$.

For preference relations with increasing impatience, the arbitrage space $[t+\rho, t]$ is bigger as increasing impatience is bigger.

The following theorem shows that TTO curves identify proneness to arbitrage in the above sense. As usual, $\tau^{*}$ is more concave than $\tau$ if there exists a concave transformation $f$ such that $\tau^{*}(t)=f(\tau(t))$ for all $t$, which holds if and only if $\frac{\tau^{* \prime \prime}}{\tau^{* \prime}} \geq \frac{\tau^{\prime \prime}}{\tau^{\prime}}$ everywhere on their domain. Note here that $\tau$ and $\tau^{*}$ are decreasing functions, for which the Pratt-Arrow index of concavity drops the minus sign relative to increasing functions. Similarly, $\tau^{*}$ is more convex than $\tau$ if there exists a convex transformation $f$ such that $\tau^{*}(t)=f(\tau(t))$ for all $t$, which holds if and only if $-\frac{\tau^{* \prime \prime}}{\tau^{* \prime}} \geq-\frac{\tau^{\prime \prime}}{\tau^{\prime}}$ everywhere on their domain. The following theorem adapts Prelec's (2004) Proposition 1 to TTO curves instead of $\ln (\varphi)$, and extends the result to increasing impatience.

Theorem 4.5 Assume that $\succcurlyeq$ and $\succcurlyeq *$ satisfy the assumptions of discounted utility of this chapter, with $\succcurlyeq$ 's TTO curve $\tau(t)$ a normalization $\frac{\ln \varphi(t)-\ln \varphi(S)}{\ln \varphi(T)-\ln \varphi(S)}$ of $\ln (\varphi(t))$ and $\succcurlyeq^{*}$ 's TTO curve $\tau^{*}(t)$ a normalization $\frac{\ln \varphi^{*}(t)-\ln \varphi^{*}\left(S^{*}\right)}{\ln \varphi^{*}\left(T^{*}\right)-\ln \varphi^{*}\left(S^{*}\right)}$ of $\ln \left(\varphi^{*}(t)\right)$, for some arbitrary $S>T$ and $S^{*}>T^{*}$.

(i) $\succcurlyeq^{*}$ exhibits more decreasing impatience than $\succcurlyeq$ if and only if $\succcurlyeq^{*}$ 's TTO curve $\tau^{*}$ is more convex than $\succcurlyeq$ 's TTO curve $\tau$.

(ii) $\succcurlyeq^{*}$ exhibits more increasing impatience than $\succcurlyeq$ if and only if $\succcurlyeq^{*}$ 's TTO curve $\tau^{*}$ is more concave than $\succcurlyeq$ 's TTO curve $\tau$.

The above theorem holds irrespective of the normalization parameters $S, T, S^{*}$, $T^{*}$ chosen. The theorem demonstrates formally that the degree of convexity of a TTO-curve determines the degree of decreasing impatience and, thus, the space for arbitrage and the proneness to anomalies as discussed by Prelec (2004) and others. From a mathematical perspective, our reformulation in terms of TTO-curves, i.e. normalized $\ln (\varphi)$ curves, may seem to be only more complex than Prelec's formulations directly in terms of $\ln (\varphi)$ itself. This reformulation is, however, the 
essential step for obtaining the empirical status of the result. The normalized curve is directly observable whereas the nonnormalized curve is not ${ }^{4}$.

\subsection{Illustrations of TTO-Sequences}

We can immediately infer proneness to time inconsistency from simply eyeballing TTO curves, curves that were obtained using only pencil and paper. Figure 4.2 displays seven TTO curves, obtained from seven subjects in the experiment described later, on normalized time intervals $\left(\tilde{t}_{0}=0, \tilde{t}_{n}=1\right)$. The curves immediately reveal that the curve of subject 7 is more convex, implying more decreasing impatience, than the curve of subject 38. Because both subjects exhibit decreasing impatience by Theorem $4.5 \mathrm{i}$, subject 7 is more prone to time inconsistency and arbitrage than subject 38. Subject 24's curve is also always below that of subject 38, suggesting more decreasing impatience. Locally around 0.45 , subject 38 exhibits more convexity though, so that this ordering of convexity does not hold on the whole interval $\left[t_{0}, t_{5}\right]$. The curves of subjects 7 and 24 intersect and there is no uniform ordering regarding their degree of nonstationarity over the whole interval $\left[t_{0}, t_{5}\right]$.

There are several concave curves suggesting increasing rather than decreasing impatience. Theorem 4.5ii shows that subject 10 is more prone to time inconsistency than subject 5 ; etc.

For sophisticated analyses, we can estimate ratios of second derivatives by first derivatives, or find best-fitting parametric curves, and compare the corresponding degrees of convexity. We can also develop global heuristic measures of convexity that can be calculated using only pencil and paper. For example, the area below the diagonal is a plausible index of convexity and of decreasing impatience. This area is a monotonic transform of the decreasing-impatience index (DI-index), defined by

$$
\text { DI-index }=\sum_{i=1}^{n-1}\left(\frac{i}{n}-\tilde{t}_{i}\right), \text { with } \tilde{t}_{i} \text { the normalization of } t_{i} .
$$

These values are 0.63 (subject 7), 0.52 (subject 24), and 0.36 (subject 38). They suggest that, overall, subject 7 exhibits more decreasing impatience than subject 24 , and subject 24 more than subject 38 . Notice that the DI-index bears some resemblance with the Gini-index in inequality measurement.

Subjects 5, 10, and 49 exhibit increasing impatience. Accordingly, their DIindices will be negative, and they are -0.26 (subject 5), -0.60 (subject 10), and

\footnotetext{
${ }^{4}$ In terms of Eq. 4.15 hereafter, Theorem 4.5 shows that we need not measure $r$, a value needed to obtain $\ln (\varphi)$ but not to obtain its normalizations.
} 


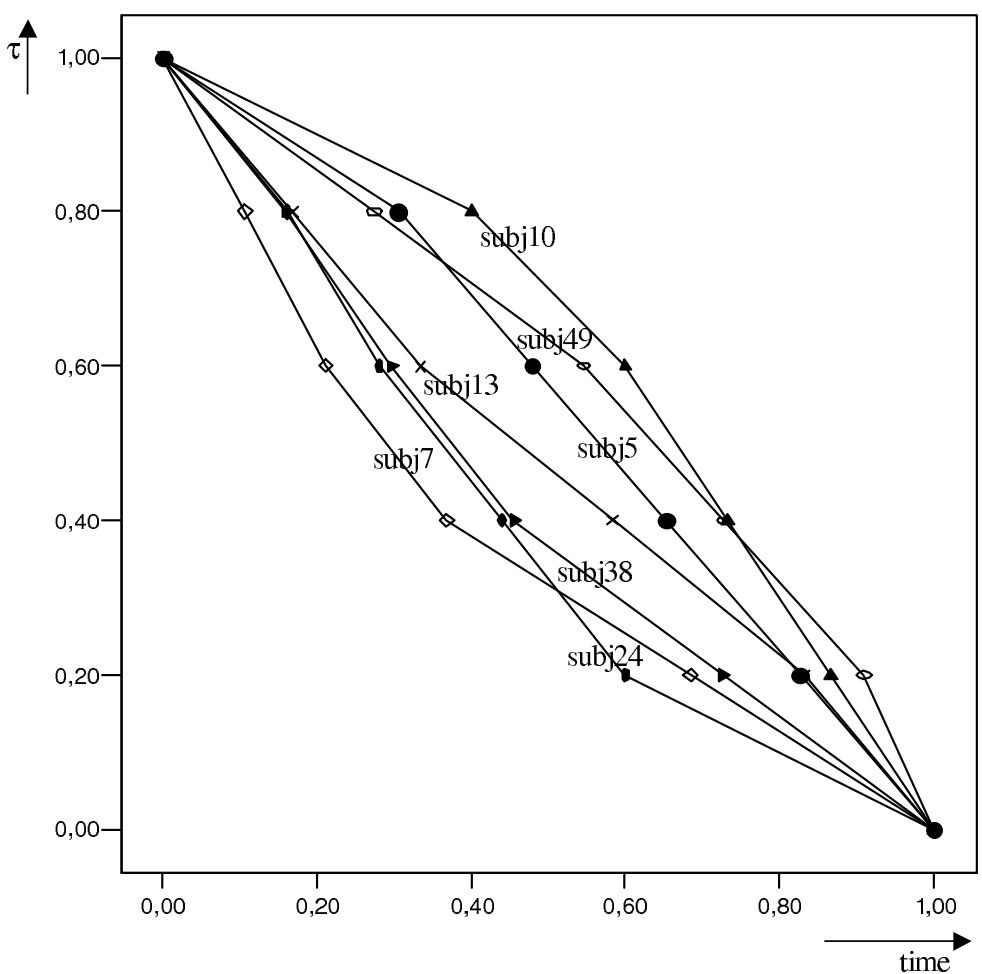

Figure 4.2: The TTO curve $\tau$ of several subjects

-0.45 (subject 49). Overall, subject 10 exhibits more increasing impatience than subject 49 , and subject 49 does so more than subject 5 .

The DI-index of subject 13 is 0.08 , and this subject virtually exhibits no overall decreasing or increasing impatience in an overall sense. Yet, this subject does deviate considerably from stationarity. For deviations from stationarity, absolute values of deviations from linearity are more relevant, with any area between the $\tau$ curve and the diagonal taken positively. We define the non-stationarity index (NS-index) as

$$
\text { NS-index }=\sum_{i=1}^{n-1}\left|\frac{i}{n}-\tilde{t}_{i}\right| .
$$

It provides an overall index of deviation from stationarity and proneness to inconsistencies without concern of the direction of deviation. For subject 13, NS-index $=$ 0.15 . To the extent that stationarity is rational, the NS-index could be interpreted as an index of irrationality. 
The DI-index and NS-index depend on the size of the interval $\left[t_{0}, t_{5}\right]$ considered in the sense that they will tend to zero if the interval $\left[t_{0}, t_{5}\right]$ becomes small. The desirability of such dependence depends on the application considered. Distortions due to this effect can be avoided by comparing subjects only on same time intervals, or taking subparts of the $\tau$ curve related to the same interval. In Figure 4.2, the curves of subjects 13 and 49 concerned similar time intervals indeed (being $[5,17]$ and $[5,16])$.

Other measures of decreasing impatience or absolute deviation from stationarity can be devised, depending on the application and the time discounting assumed. Let us consider generalized hyperbolic discounting, $\varphi(t)=(1+h t)^{-r / h}$ (Loewenstein and Prelec, 1992), with $0 \leq h<\infty$ an index of decreasing impatience, and with stationarity and constant discounting $e^{-r t}$ the limiting case of $h \rightarrow 0$. This family incorporates most of the popular hyperbolic families other than quasi-hyperbolic discounting, such as those of Mazur (1987) and Harvey (1986, 1995). Rohde (2005) derived the most appropriate index of convexity for this family, called the hyperbolic factor. For a TTO sequence $t_{0}, \ldots, t_{n}$, hyperbolic factors can be calculated as

$$
\text { hyperbolicfactor }(i, j)=\frac{\left(t_{j}-t_{i}\right)-\left(t_{j-1}-t_{i-1}\right)}{t_{i}\left(t_{j-1}-t_{i-1}\right)-t_{i-1}\left(t_{j}-t_{i}\right)}
$$

for $j>i$. Hence, for one TTO sequence with $n=5,10(=4+3+2+1)$ hyperbolic factors can be calculated. As Rohde (2005) demonstrated, for generalized hyperbolic discounting, $\varphi(t)=(1+h t)^{-r / h}$, the hyperbolic factor is constant, independent of $i$ and $j$ or the time-tradeoff sequence considered, and is always equal to $h$. For constant discounting (stationarity), the hyperbolic factor will always be zero, and for quasi-hyperbolic discounting the hyperbolic factor is positive at timepoint 0 and zero for all future timepoints. Thus, this statistic can readily serve to test these models.

The relation between Prelec's convexity index and the hyperbolic factor can be compared with indices of risk aversion of utility functions $U$ in expected utility. The absolute Pratt-Arrow index $-U^{\prime \prime} / U^{\prime}$ is most appropriate for so-called CARA utility, but the relative index $x\left(-U^{\prime \prime} / U^{\prime}\right)$ is most appropriate for so-called CRRA utility. Thus, what is the most useful index depends on the application mentioned. Theorem 4.5 described cases where Prelec's measure is most suited. If we restrict our attention to generalized hyperbolic discounting, the hyperbolic factor is useful.

The hyperbolic factor can be directly calculated from TTO sequences, and can be used to test whether generalized hyperbolic discounting holds and, if it does, to distinguish between its various subfamilies. One necessary condition for 
generalized hyperbolic discounting to hold, and for $h$ to be well behaved, is that the denominator in Eq. 4.12 be positive, i.e.

$$
t_{i}\left(t_{j-1}-t_{i-1}\right)-t_{i-1}\left(t_{j}-t_{i}\right)>0 \text { for } j>i .
$$

This inequality provides a test of generalized hyperbolic discounting, as does constantness of the hyperbolic factor when defined.

\subsection{Deriving the Discount Function from TTO- Sequences}

We saw in preceding sections that TTO sequences give the normalized logarithm of the discount function, and they give the degree of change of impatience and discounting through the degree of convexity of the function obtained. We did not derive the complete discount function in the preceding section, because we did not establish the rate of time preference in any absolute sense. Deriving the complete discount function from TTO sequences is the purpose of this section. One way to identify the discount function is to derive the utility function from some extra information or from some extra assumption, such as linearity as is often done in the literature. Then we can use Eq. 4.3 and we readily get $\varphi$.

An alternative route that does not need any assumption about utility is as follows. We can take any indifference between outcome streams with two nonzero outcomes:

$$
(b: \gamma, c: \gamma) \sim(a: \gamma, d: \gamma) \text { for } \gamma>0 \text { and } a<b<c<d
$$

We give the proof of the following observation in the main text because it demonstrates how the discount function can be calculated from Eq. 4.14 together with TTO sequences.

Observation 4.6 Given the TTO-curve $\tau$, the discount function $\varphi$ is uniquely determined through one observed indifference 4.14.

Proof

Let $t_{n}>t_{0}$, and assume that

$$
\tau(t)=\frac{\ln (\varphi(t))-\ln \left(\varphi\left(t_{n}\right)\right)}{\ln \left(\varphi\left(t_{0}\right)\right)-\ln \left(\varphi\left(t_{n}\right)\right)}
$$

That is, $\tau$ is a normalization of $\ln (\varphi(t))$. There exist, as yet unknown, parameters 
$\lambda$ and $r$ such that $\ln (\varphi(t))=\lambda+r \tau(t)$, i.e. ${ }^{5}$

$$
\varphi(t)=e^{\lambda} \times\left(e^{\tau(t)}\right)^{r} .
$$

The parameter $e^{\lambda}$ is an arbitrary scaling constant without empirical implications. We may as well assume that it is $e^{-r}$. The power $r$ determines the rate of time preference and is empirically relevant. For example, if we let the irrelevant factor $e^{\lambda}$ be $e^{-r}$ and rewrite $\varphi(t)$ as $e^{-r} \times\left(e^{r}\right)^{\tau(t)}$, then for the special case of constant discounting and linear $\tau(t)=1-t, e^{-r}$ is the discount factor.

TTO sequences in isolation cannot identify the power $r$ and, thus, cannot identify the absolute rate of time preference. To see this point, note that TTOsequences concern simple outcome streams. If $\varphi(t) U(x)$ represents preferences over simple outcome streams $(t: x)$, then so does $\varphi(t)^{r} U(x)^{r}$ for every $r>0$, because $\varphi(t) U(x) \geq \varphi(s) U(y)$ if and only if $\varphi(t)^{r} U(x)^{r} \geq \varphi(s)^{r} U(y)^{r}$. Hence, without any assumption about utility, simple outcome streams and TTO-sequences cannot identify the power of time discounting and the absolute degree of discounting, indeed.

The indifference in Eq. 4.14 implies that $\varphi(b) U(\gamma)+\varphi(c) U(\gamma)=\varphi(a) U(\gamma)+$ $\varphi(d) U(\gamma)$, or $\varphi(b)+\varphi(c)=\varphi(a)+\varphi(d)$. Substituting Eq. 4.15 and dropping $e^{\lambda}$ gives

$$
\left(e^{\tau(b)}\right)^{r}+\left(e^{\tau(c)}\right)^{r}=\left(e^{\tau(a)}\right)^{r}+\left(e^{\tau(d)}\right)^{r} .
$$

It is well-known that for all quadruples $a^{\prime}\left(e^{\tau(a)}\right.$ above), $b^{\prime}\left(e^{\tau(b)}\right.$ above $), c^{\prime}\left(e^{\tau(c)}\right.$ above), and $d^{\prime}\left(e^{\tau(d)}\right.$ above) with $a^{\prime}>b^{\prime}>c^{\prime}>d^{\prime}$ (recall that $\tau$ is decreasing) there exists a unique real number $r$ such that exactly one of the following equations holds:

$$
\begin{aligned}
b^{\prime r}+c^{\prime r} & =a^{\prime r}+d^{\prime r} \text { with } r>0 \\
\ln \left(b^{\prime}\right)+\ln \left(c^{\prime}\right) & =\ln \left(a^{\prime}\right)+\ln \left(d^{\prime}\right), \text { corresponding to } r=0 \\
b^{\prime r}+c^{\prime r} & =a^{\prime r}+d^{\prime r} \text { with } r<0
\end{aligned}
$$

Such equations have, for instance, been studied in decision under risk with expected utility where $\left(b^{\prime}, c^{\prime}\right)$ and $\left(a^{\prime}, d^{\prime}\right)$ designate fifty-fifty lotteries for money, and constant relative risk averse utility $U(x)=x^{r} / r(U(x)=\ln (x)$ for $r=0)$ is used to fit data. Unfortunately, there is no analytic expression for the solution $r$ to the best of our knowledge, but $r$ can readily be determined numerically.

In our above analysis for time preference, only positive powers $r\left(=\ln \left(\varphi\left(t_{0}\right)\right)-\right.$ $\left.\ln \left(\varphi\left(t_{n}\right)\right)\right)$ are possible. In the experiment we measured Eqs. 4.14 empirically, and

\footnotetext{
${ }^{5}$ We have $\lambda=\ln \left(\varphi\left(t_{n}\right)\right)$ and $r=\ln \left(\varphi\left(t_{0}\right)\right)-\ln \left(\varphi\left(t_{n}\right)\right)$, with $\varphi\left(t_{0}\right)$ and $\varphi\left(t_{n}\right)$ unknown.
} 
then solved for $r$. If negative $r$ resulted, it followed that the discounted utility model was falsified. To clarify how such a violation can arise, assume a TTO sequence $t_{0}, \ldots, t_{5}(n=5)$. Assume that we take $a=t_{1}, b=t_{2}$, and $c=t_{3}$, in Eq. 4.14, and the subject chooses $d<t_{4}$. Then $\varphi\left(t_{2}\right)+\varphi\left(t_{3}\right)=\varphi\left(t_{1}\right)+\varphi(d)>$ $\varphi\left(t_{1}\right)+\varphi\left(t_{4}\right)$, so that $\varphi\left(t_{1}\right)-\varphi\left(t_{2}\right)<\varphi\left(t_{3}\right)-\varphi\left(t_{4}\right)$. This contradicts the equality $\varphi\left(t_{3}\right)-\varphi\left(t_{4}\right)=\mu^{2}\left(\varphi\left(t_{1}\right)-\varphi\left(t_{2}\right)\right)$ for $0<\mu=\varphi\left(t_{2}\right) / \varphi\left(t_{0}\right)<1$, and the general discounted utility model has been falsified.

Figure 4.3 depicts a discount function that we obtained for subject 5 . We used his indifferences $(0: 700) \sim(6: 900),(6: 700) \sim(12: 900),(12: 700) \sim(16: 900)$, $(16: 700) \sim(20: 900),(20: 700) \sim(24: 900)$, which yields the TTO sequence $t_{0}=0, t_{1}=6, t_{2}=12, t_{3}=16, t_{4}=20$, and $t_{5}=24$. Further we used his indifference $(12: 700,16: 700) \sim(6: 700,24: 700)$ as a version of Eq. 4.14 to estimate $r$ in

$$
\left(e^{\tau(12)}\right)^{r}+\left(e^{\tau(16)}\right)^{r}=\left(e^{\tau(6)}\right)^{r}+\left(e^{\tau(24)}\right)^{r} .
$$

Eq. 4.20 is equivalent to

$$
\left(e^{3 / 5}\right)^{r}+\left(e^{2 / 5}\right)^{r}=\left(e^{4 / 5}\right)^{r}+\left(e^{0}\right)^{r} .
$$

We obtained an estimated power $r=1.41$.

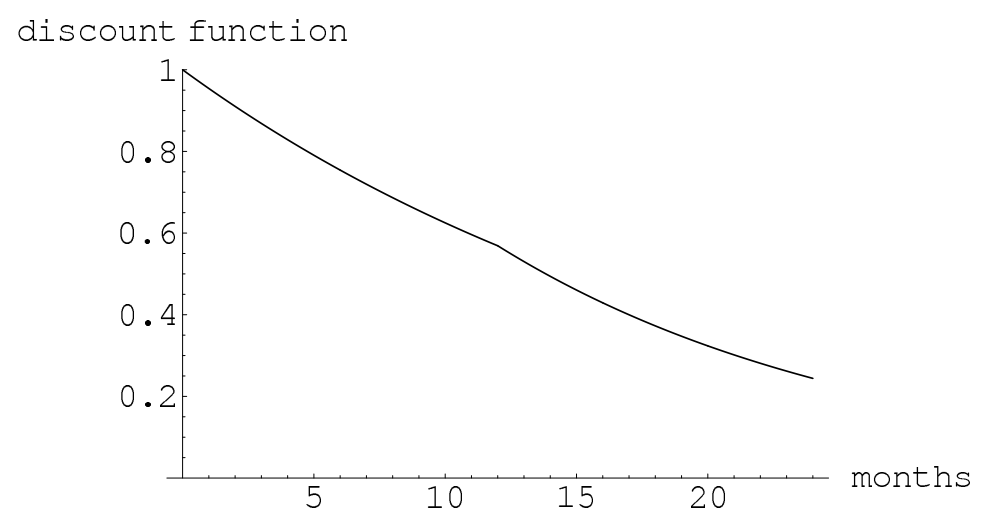

Figure 4.3: The discount function $\varphi(t)$ of subject 5 
Table 4.1: Parameters for the four TTO sequences

\begin{tabular}{llll}
\hline \hline sequences & $t_{0}$ & $\beta$ & $\gamma$ \\
\hline I & 0 months & $€ 700$ & $€ 900$ \\
II & 0 months & $€ 2800$ & $€ 3300$ \\
III & 5 months & $€ 700$ & $€ 900$ \\
IV & 0 months & $€ 1600$ & $€ 1900$ \\
\hline \hline
\end{tabular}

The outcomes $\beta, \gamma$, and the initial timepoints $t_{0}$ are as in Eq. 4.2.

\subsection{Method of Experiment}

Participants. $\mathrm{N}=55$ subjects took part. There were 31 students from Erasmus University, of whom 21 were from finance or economics and the others were from various other disciplines, and there were 24 students from Maastricht University, with only 2 students from economics or finance and the rest from various disciplines.

Motivating subjects. Every subject received $€ 10$ for participating. All payoffs in the stimuli were hypothetical. This point is discussed in Section 4.8.

Procedure. The experiment was run by computer, and subjects were interviewed individually. On average, the task took 15 minutes per subject. We ran extensive pilots with 53 subjects in order to determine the appropriate setup of the experiment.

We took one month as unit of time. Subjects first went through a training phase, where preferences $(0: 700) \prec(1: 900)$ and $(0: 700) \succ(600: 900)$ were mostly observed (with sometimes one or both reversed). Then, in a training matching task, we asked for the value $t$ to give the indifference $(0: 700) \sim(t: 900)$, and then for the value $t$ to give the indifference $(0: 2800) \sim(t: 3300)$.

Stimuli. We elicited four TTO sequences for each subject (Table 4.1). Every sequence consisted of 5 steps, i.e. $n=5$. All tasks were matching tasks, similar to the last task of the training phase.

The computer screen was as given in the Appendix. The pilots suggested that a direct successive elicitation of the timepoints $t_{1}, \ldots, t_{5}$ of one TTO sequence could generate order effects. Hence, in the main experiment we first elicited $t_{1}$ for every TTO sequence, next $t_{2}$ for every TTO sequence, etc.

We elicited two versions of Eq. 4.14. In both we took $\gamma=€ 700$. In the first 
we measured an indifference

$$
(5: 700,11: 700) \sim(1: 700, T: 700) .
$$

where subjects were asked to provide their indifference value $T$ through a matching question. In the second we measured an indifference

$$
\left(t_{2}: 700, t_{3}: 700\right) \sim\left(t_{1}: 700, T: 700\right),
$$

where $t_{1}, t_{2}$, and $t_{3}$ were from the elicited TTO sequence I.

Demographic variables. At the end of the experiment, subjects were asked to report their gender, age, length, weight, field of studies, nationality, and also whether or not they smoked. We also asked seven behavioral questions on a scale from 1 to 7 , where 1 means totally disagree and 7 means totally agree. The questions concerned behavioral aspects that we thought could have an influence on discounting and are given in the Appendix.

Analysis. We did all tests both parametrically and non-parametrically. These always gave similar results, and we only report the non-parametric tests.

Analysis of Group Averages. Changes in WTW indicate whether subjects satisfy constant, decreasing or increasing impatience. We tested for constant WTW for each TTO sequence separately using a Friedman test.

Next, for every two subsequent measurements of WTW $\left(d_{i}\right.$ and $\left.d_{i-1}\right)$ we tested equality using Wilcoxon tests. We also tested equality of WTW between the first questions of sequence I $((0: 700) \sim(t: 900))$ and of sequence III $((5: 700) \sim$ $(t: 900))$. Because these concern the same outcomes, stationarity predicts the same WTW here. We also checked whether the temporal attitude suggested by this comparison is consistent with the temporal attitude suggested by comparisons within sequence I. That is, we checked whether the change in WTW from the first question of sequence I to the first question of sequence III has the same sign as the first change in WTW within sequence I.

Analyses of Individual Data. A subject was classified as exhibiting increasing (constant, decreasing) impatience if at least $50 \%$ of her changes in WTW suggested so, where we considered all sequences together. A double classification as constant and increasing (decreasing) was reclassified as increasing (decreasing), and a double classification as increasing and decreasing was taken as unclassified, as were all other cases. We used these conservative criteria to reduce the effects of response error. Such a threshold of $50 \%$ has been used before in the literature (Abdellaoui, 2000). We tested whether significantly more subjects are classified as increasingly or decreasingly impatient using Wilcoxon. 
Next, we tested whether quasi-hyperbolic discounting holds. For every subject we split all changes in WTW of all TTO sequences into two groups: the group containing all changes in WTW where the first timepoint was 0 , and the group containing the rest. For both groups, we chose the same $50 \%$ classification as before. Under quasi-hyperbolic discounting, the WTW should increase in the former group and be constant in the latter. We performed similar Wilcoxon tests as before.

For every subject we calculated indices of decreasing impatience and of nonstationarity, and also the hyperbolic factors as explained in Section 4.5. We compared the indices of all subjects between sequences by means of Wilcoxon signed rank tests. To test for a possible special effect of first questions, we also considered sequences with the first step left out. We computed the DI-index for these reduced sequences as follows: DI-index $=\sum_{i=1}^{3}\left(\frac{i}{4}-\tilde{t}_{i+1}\right)$, with $\tilde{t}_{i}$ the normalization of $t_{i}$ such that $\tilde{t}_{1}=0$ and $\tilde{t}_{5}=1$.

Next, we regressed the indices of decreasing impatience and non-stationarity on the body-mass index. We estimated the correlation between each of the seven behavioral questions and each DI-index and each NS-index. We also regressed the mean of the DI-indices per subject and that of the NS-indices on gender, smoker, age, length, weight, and all behavioral questions together. Finally, we estimated the power of discounting $r$ in Eq. 4.15 from the questions in Eqs. 4.22 and 4.23.

\subsection{Results}

\section{Group Averages}

Figure 4.4 gives the TTO curves constructed from the medians of the answers of all subjects. The curves suggest that subjects are increasingly impatient in the beginning and near future, and constantly impatient thereafter. Statistical analyses confirm this pattern. The Friedman tests rejected constantness of the WTW $(p<0.01)$ for all sequences. We repeated the test with the first WTW excluded. As expected, then the null hypothesis of constant WTW is not rejected ( $p>0.20$ for all tests). Thus, our findings suggest that people satisfy stationarity for timepoints beyond a certain threshold. From the third sequence we can see that this threshold exceeds 5 months.

Figure 4.5 shows median WTWs. The vertical axes all have the same scale and give the WTW. We can clearly see that the WTW drops in the beginning and remains more or less constant later on for every sequence. This is confirmed by Wilcoxon tests. The results of the Wilcoxon test are summarized in Table 4.2. The 


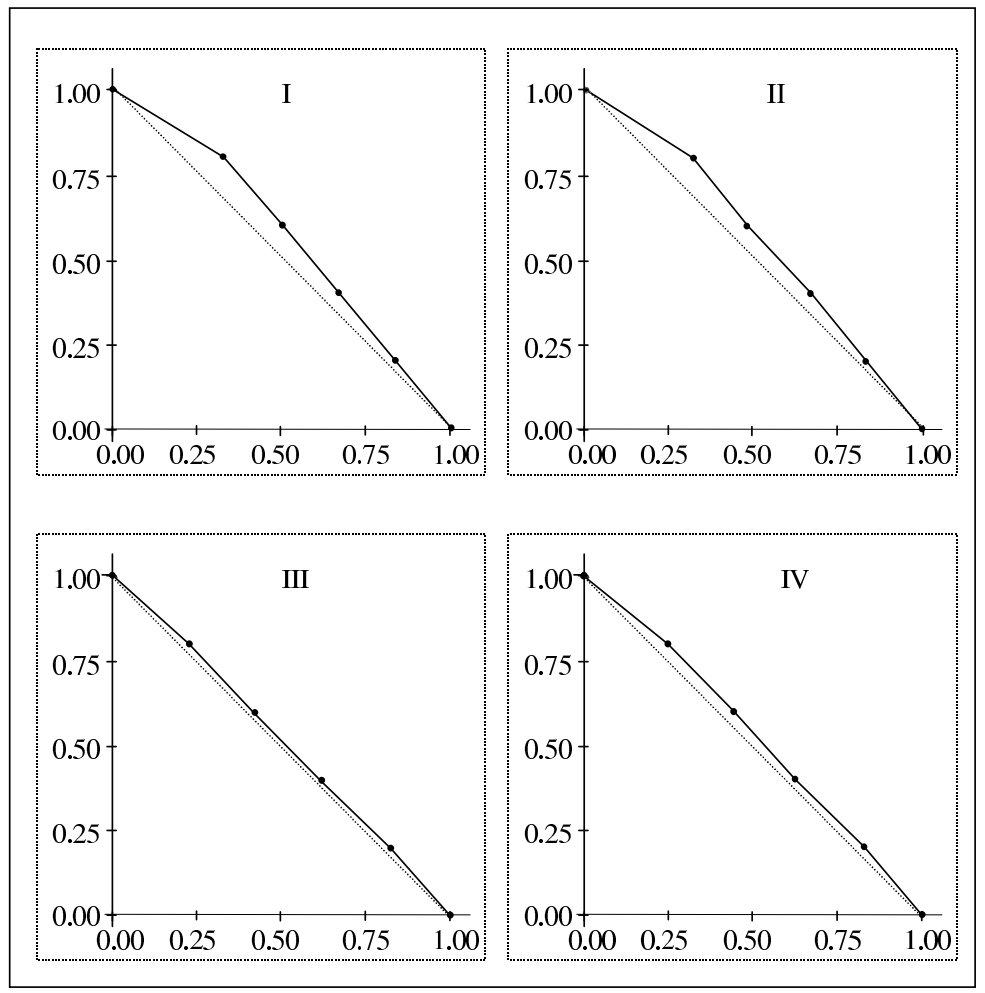

Figure 4.4: The TTO curves $\tau$ for median answers of the four TTO sequences

WTW changed significantly in the first steps $\left(d_{2}-d_{1}\right)(\alpha=0.01)$. The WTW decreases there, suggesting increasing impatience. The WTW increases in the second step $\left(d_{3}-d_{2}\right)$ for sequence III $(\alpha=0.05)$. No other changes are significant at $\alpha=0.05$.

A Wilcoxon test shows that the first WTW of the third sequence is significantly lower $(p<0.01)$ than the first WTW of the first sequence. Thus, subjects are consistent between sequences I and III.

\section{Individual Data}

The individual data confirm the preceding findings. Subjects are increasingly impatient for timepoints close to 0 and constantly impatient for later timepoints, as follows. The classification of all subjects based on all sequences together yields 18 subjects exhibiting constant impatience, 3 exhibiting decreasing, 10 exhibiting increasing impatience, and 24 not classified (Table 4.3). Thus, based on this classification we cannot say much about the behavior of individual subjects. The 


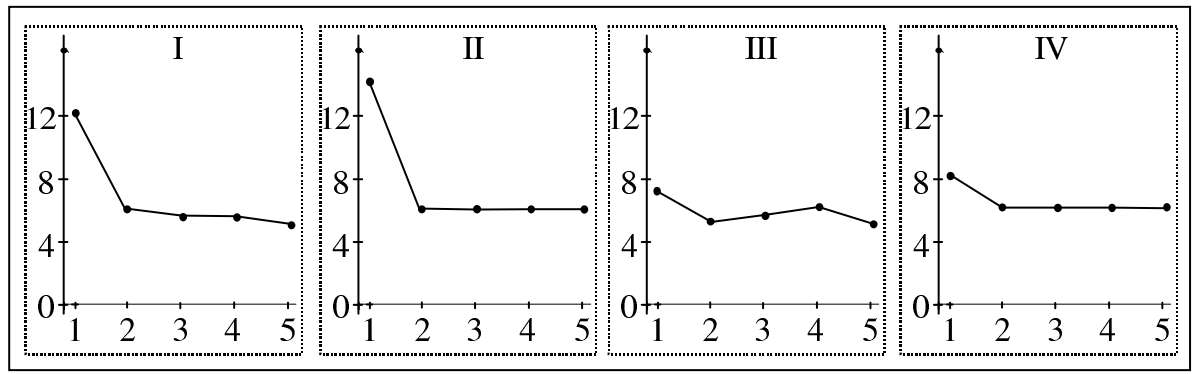

Figure 4.5: Median willingness to wait for each sequence

Table 4.2: Wilcoxon signed rank tests: Z (p-value, 2-tailed)

\begin{tabular}{lllll}
\hline \hline & & \multicolumn{2}{c}{ WTW } \\
\hline Seq. & $d_{2}-d_{1}$ & $d_{3}-d_{2}$ & $d_{4}-d_{3}$ & $d_{5}-d_{4}$ \\
\hline I & $-4.40(0.000)$ & $-0.51(0.612)$ & $-0.63(0.531)$ & $-0.34(0.732)$ \\
II & $-4.50(0.000)$ & $1.35(0.176)$ & $-0.93(0.352)$ & $-0.98(0.329)$ \\
III & $-3.39(0.001)$ & $2.00(0.046)$ & $-0.29(0.769)$ & $-0.95(0.341)$ \\
IV & $-3.19(0.001)$ & $1.03(0.302)$ & $-0.41(0.681)$ & $1.05(0.293)$ \\
\hline \hline
\end{tabular}

Wilcoxon test shows that there is more tendency towards increasing than towards decreasing impatience $(p=0.052)$. In the group of all questions with a first timepoint zero, 8 subjects exhibit constant impatience, 3 subjects exhibit decreasing impatience, 36 subjects exhibit increasing impatience, and 8 subjects could not be classified. This suggests that most subjects indeed are increasingly impatient for timepoint zero, which is supported by the Wilcoxon test $(p=0.000)$. In the other group (first timepoint positive), 21 subjects exhibit constant impatience, 5 subjects exhibit decreasing, 6 subjects exhibit increasing, and 23 subjects could not be classified. Thus, it appears that most subjects indeed exhibit constant impatience for timepoints not too close to 0 .

Calculations of the hyperbolic factors revealed that Eq. 4.13 was widely violated, for virtually all subjects in many questions. This falsifies the generalized hyperbolic discounting model, and Rohde's hyperbolic factor cannot be calculated in many situations.

In view of the problems of calculating the hyperbolic factors, we only use the indices of decreasing impatience (DI-index) and of non-stationarity (NS-index) to compare subjects. The medians of the indices of decreasing impatience over 
Table 4.3: Classification of individuals

\begin{tabular}{lcccc}
\hline \hline & \multicolumn{4}{c}{ Impatience } \\
\hline Questions & Constant & Decreasing & Increasing & unclassified \\
\hline all & 18 & 3 & 10 & 24 \\
timepoint 0 & 8 & 3 & 36 & 8 \\
timepoint $>0$ & 21 & 5 & 6 & 23 \\
\hline \hline
\end{tabular}

the whole sequences are significantly negative $(p<0.01)$ so that subjects are increasingly impatient overall. The medians of the DI-index were respectively $-0.33,-0.28,-0.092$, and -0.19 . We observe that the third sequence had both a lower NS-index and a lower absolute value of the DI-index. This is probably caused by the fact that the third sequence starts closer to the threshold from whereon subjects satisfy constant impatience. The DI-indices of the reduced sequences, the sequences without the first steps, did not deviate significantly from zero, indicating that the increasing impatience found earlier is indeed due to the first step of every sequence.

We proceed by considering only the DI-index and NS-index of the complete sequences. Based on a Wilcoxon signed rank test, the DI-index and the NS-index are significantly different for every sequence $(p<0.01)$, where the NS-index is always larger than minus the DI-index. Since most indices of decreasing impatience are negative, this finding implies that for most subjects the TTO curve $\tau$ intersects the curve belonging to a linear TTO curve at least once. Thus, most subjects are not clearly either increasingly or decreasingly impatient, but are a mix of both.

There was no significant difference in DI-index and NS-index between sequences I and II and between sequences III and IV. For all other pairs of sequences, the sequence with the higher sequence number provided significantly higher DI-indices and significantly lower NS-indices than the ones before $(p<0.01$ for all but one, $p<0.05$ for all). Thus, subjects became less non-stationary and more decreasingly impatient or, equivalently, less increasingly impatient in later sequences.

On average, men had higher DI-indices and lower NS-indices, except for the DI-index in sequence III, but the differences were usually not significant, with marginal significance $(p<0.10)$ for the DI-index of sequence I and for the NSindices of sequences I and II, and significance $(p<0.05)$ only for the NS-index of sequence IV.

We found no significant relations between our indices and demographic vari- 
ables otherwise. Also, the correlations between the behavioral questions and the indices were mostly insignificant, so this gives no clear indication that the behavioral questions predict behavior. In the two regressions of the mean of the DI-indices per subject and that of the NS-indices on gender, smoker, age, length, weight, and all behavioral questions together, only the coefficient on the second behavioral question with the mean of the NS-index as dependent variable, was significantly positive $(p=0.045)$ and all other coefficients were insignificant.

In calculations of the power $r$ in Eq. 4.15 for Eqs. 4.22 and 4.23 , about $1 / 3$ of these were negative. It shows that there are many violations of the basic model of general discounting.

\subsection{Discussion}

Our findings suggest that the subjects satisfy increasing impatience in the beginning, and constant impatience thereafter. Thus, we find a kind of "reversed quasihyperbolic" discounting, where impatience is constant after a certain threshold and increasing as opposed to decreasing in the beginning. Impatience, however, continues to increase up to 5 months and is not constant immediately after the present. Informal discussions with subjects indicated that they understood the questions and knew what they wanted to answer. For the major finding of this study that deviates from common empirical findings in the literature, i.e. increasing instead of decreasing impatience, there was clear support from the informal discussions. Many students indicated that they did not mind a delay at first, but after a long wait they extra disliked further delays. This finding is opposite to subjects' becoming more insensitive to delays, as is commonly assumed in the literature.

Our finding of increasing impatience are consistent with several other studies (Airoldi, Read, and Frederick, 2005; Frederick, 1999; Read, Airoldi, and Loewe, 2005; Read, Frederick, Orsel, and Rahman, 2005; Rubinstein, 2003; Sayman and Öncüler, 2005). Read, Frederick, Orsel, and Rahman (2005) found that hyperbolic discounting is only observed when time is described in delay terms as opposed to calendar time terms. Rubinstein (2003) reported three experiments that provide evidence against constant or decreasing impatience. Bommier (2005) and Dasgupta and Maskin (2005) gave theoretical reasons why increasing impatience can occur.

The setup of the experiment made it unlikely that subjects noticed that the questions were chained, and that several of them together served to elicit sequences. 
Therefore, it is unlikely that order effects would cause the increasing impatience we found.

For the violations of general discounted utility that we found when estimating the powers in Eq. 4.15, it is likely that the time-separability assumption underlying general discounted utility is violated. It plays no role for the measurements of TTO sequences, and only becomes effective for two or more outcomes. This finding adds to the motivation for paying attention as much as possible to simple outcome streams, as done when measuring TTO curves. Therefore the analysis of the TTO sequences minimizes the biases caused by this violation.

Subjects were paid a flat fee for participating and all questions were hypothetical. There are several reasons why we did not use performance-based real incentives. First, it is administratively complicated to transfer money on the timepoints specified, not only for the experimenters but also for the subjects. Hence, such a procedure will generate many extra biases such as through doubts on the subjects' part about reliable implementations. Second, the outcomes we used were large so as to avoid subjects thinking that the amount of money is trivial anyhow and not worth thinking about carefully. Then real payments make the experiment prohibitively expensive. Also, no clear evidence exists that hypothetical amounts are discounted differently than real amounts (Frederick, Loewenstein, and O'Donoghue, 2002). In other fields with stimuli that are not cognitively demanding similar to our study, hypothetical incentives do not seem to give qualitatively different results, although real incentives tend to reduce data variability (Camerer and Hogarth, 1999, and Hertwig and Ortmann, 2001). Finally, there is no clear incentive for our subjects to please the experimenter, as there can be in experiments about social behavior.

Many studies that provide evidence in favor of decreasing impatience elicit indifference values in the outcome domain. They fix two timepoints and one outcome and elicit a second outcome that makes the subject indifferent between the two simple outcome streams. We elicit indifference values in the time domain. Because we are interested in properties of the discount function, and not of the utility function, it is more natural to focus the subjects' attention on this dimension as our questions did. Because, by construction of a TTO sequence, utilities cancel out from the equations, our method does not require richness in the outcome dimension and can, for instance, be used with qualitative health outcomes. It naturally exploits the richness in the time dimension that is available anyhow.

Scale compatibility entails that subjects put more weight on the time dimension in our setup than in studies eliciting indifferences in the outcome domain. This could mean that subjects discount outcomes more heavily in our setup but it need 
not affect the main topic of interest to us: nonconstant impatience.

Although eliciting indifferences has not been very common in the time domain, it has been used on a number of occasions, for instance by Mazur (1987). He conducted experiments with pigeons instead of humans. Green, Fristoe, and Myerson (1994) did similar experiments with humans. These studies, as our study, exploited the richness of the time dimension to study temporal preference. They, however, still assumed linear utility of money.

Our findings suggest a number of new directions for intertemporal preference. Virtually all existing models, including quasi-hyperbolic discounting and generalized hyperbolic discounting, assume universal decreasing or constant impatience, and have no clear extension to allow for increasing impatience. However, even if group averages satisfy decreasing impatience, then there will still be individuals who exhibit increasing impatience, so that for any data fitting at the individual level such functions are required. For this reason we could not implement the planned test to discriminate which of the currently popular models fit the data better: None of them could at all fit data. In particular Rohde's (2005) hyperbolic factor, in theory a good tool to empirically distinguish between various families, was not defined for many answers of virtually all subjects. Also when we used TTO sequences to derive discount functions from two nonzero-outcome streams, our findings were mostly negative: We found the general discounted utility model (4.1) extensively violated. Hence, the development of models relaxing this assumption is also desirable.

\subsection{Appendix}

Proof of TheOrem 4.5. Because $\tau$ and $\tau^{*}$ are strictly decreasing functions, $\tau^{*}(t)=f(\tau(t))$ for a strictly increasing function $f$. Take any intervals $[d, c]$ and $[b, a]$ to the right of $[d, c](b>d$ and $a>c)$ in the domain of $f$. Then $a=\tau(s)$, $b=\tau(t), c=\tau(s+\sigma)$, and $d=\tau(t+\sigma+\rho)$ for some $s<t, s+\sigma<t+\sigma+\rho$, $\sigma>0, \sigma+\rho>0$. Because the ranges of $U$ and $U^{*}$ contain nondegenerate intervals with 0 as lower bound, there exist outcomes $\beta<\gamma$ with

$$
(s: \beta) \sim(t: \gamma)
$$

and outcomes $\beta^{*}<\gamma^{*}$ with

$$
\left(s: \beta^{*}\right) \sim^{*}\left(t: \gamma^{*}\right)
$$

(Here is where we crucially use continuity of utility.) Only the utility ratios $U(\beta) / U(\gamma)$ and $U^{*}\left(\beta^{*}\right) / U^{*}\left(\gamma^{*}\right)$ matter for all that follows and, hence, the par- 
ticular choices of $\beta, \gamma, \beta^{*}, \gamma^{*}$ are immaterial for all that follows.

We have equivalence of the following statements:

$$
\begin{aligned}
a-b & =c-d ; \\
\tau(s)-\tau(t) & =\tau(s+\sigma)-\tau(t+\sigma+\rho) ; \\
\ln \varphi(s)-\ln \varphi(t) & =\ln \varphi(s+\sigma)-\ln \varphi(t+\sigma+\rho) ; \\
\varphi(s) / \varphi(t) & =\varphi(s+\sigma) / \varphi(t+\sigma+\rho) ; \\
(s+\sigma: \beta) & \sim(t+\sigma+\rho: \gamma) .
\end{aligned}
$$

We also have logical equivalence of the following statements:

$$
\begin{aligned}
f(a)-f(b) & \geq f(c)-f(d) ; \\
\tau^{*}(s)-\tau^{*}(t) & \geq \tau^{*}(s+\sigma)-\tau^{*}(t+\sigma+\rho) ; \\
\ln \varphi^{*}(s)-\ln \varphi^{*}(t) & \geq \ln \varphi^{*}(s+\sigma)-\ln \varphi^{*}(t+\sigma+\rho) ; \\
\varphi^{*}(s) / \varphi^{*}(t) & \geq \varphi^{*}(s+\sigma) / \varphi^{*}(t+\sigma+\rho) ; \\
\left(s+\sigma: \beta^{*}\right) & \preccurlyeq^{*}\left(t+\sigma+\rho: \gamma^{*}\right) .
\end{aligned}
$$

It is well-known that $f$ is convex if and only if for all $a, b, c, d$ as above we have $f(a)-f(b) \geq f(c)-f(d)$. As we have just demonstrated, this is, in view of Eq. 4.24 and Eq. 4.25 and the independence of the choices $\beta, \gamma, \beta^{*}, \gamma^{*}$ above, the same as the requirement that $(s+\sigma: \beta) \sim(t+\sigma+\rho: \gamma)$ imply $\left(s+\sigma: \beta^{*}\right) \preccurlyeq^{*}\left(t+\sigma+\rho: \gamma^{*}\right)$ for all $s, t, \sigma, \rho$ as above. That is, convexity of $f$ is equivalent to more decreasing impatience for $\succcurlyeq^{*}$ than for $\succcurlyeq$. Reversing inequalities and weak preferences above shows that concavity of $f$ is equivalent to more increasing impatience for $\succcurlyeq^{*}$ than for $\succcurlyeq$.

The seven behavioral questions were as follows:

1. I do not study regularly, but often postpone it for too long, so that the exams-week is extra stressful.

2. I wish I would drink less alcohol per week than I do currently.

3. I wish I would eat less per day than I do currently.

4. I tend to postpone things.

5. I am impatient. 
6. I am often late.

7. I tend to do impulsive purchases.

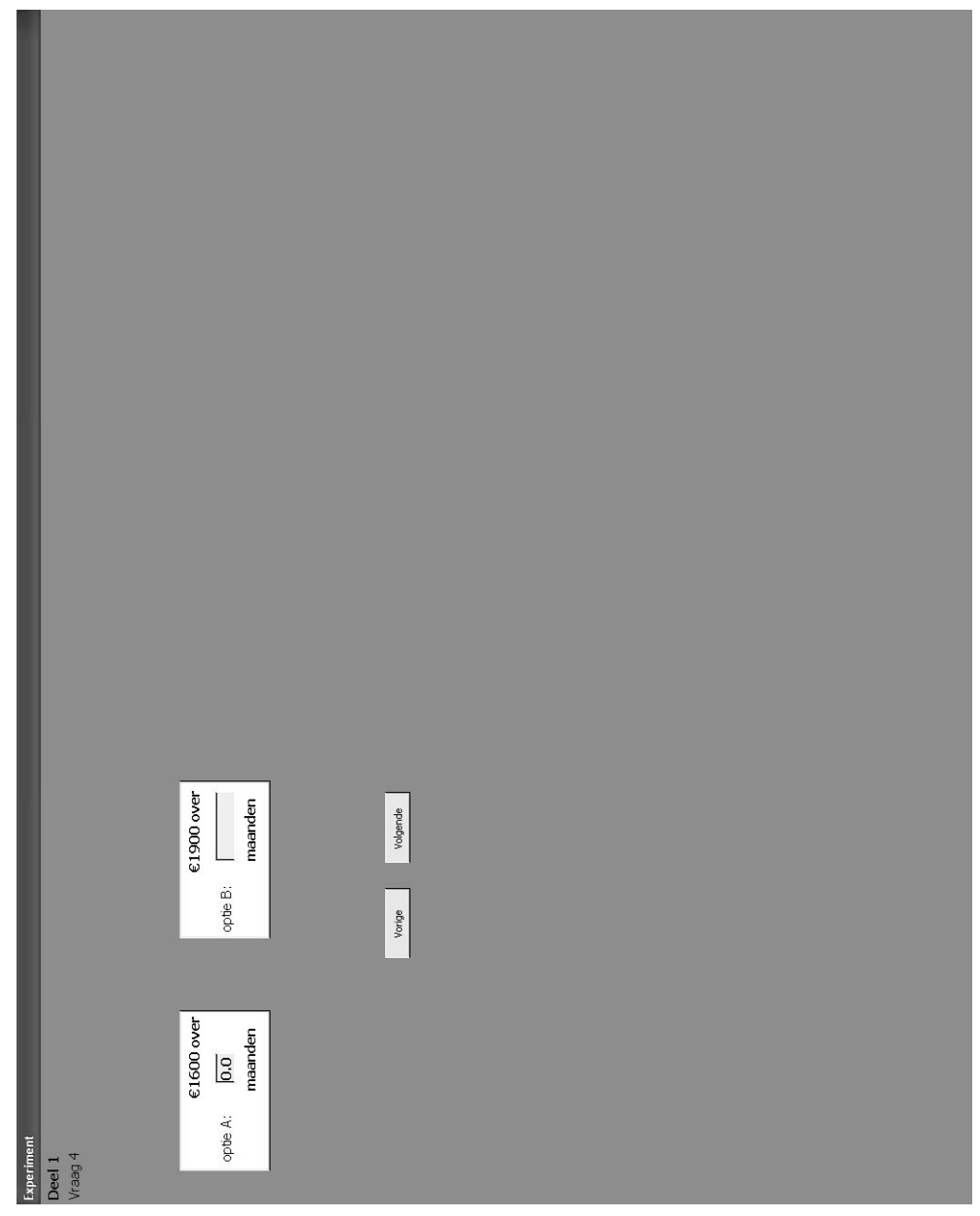

Figure 4.6: Layout of the computer screen 



\section{Part II}

\section{General Equilibrium}





\section{5}

\section{Time-Inconsistent Preferences in A General Equilibrium Model}

This chapter ${ }^{1}$ introduces time-inconsistent preferences in a multicommodity general equilibrium framework with incomplete markets. It is based on Herings and Rohde (2006). The standard concept of competitive equilibrium is extended in order to allow for changes in intertemporal preferences. Depending on whether or not agents recognize that their intertemporal preferences change, agents are called sophisticated or naïve. This chapter presents competitive equilibrium notions for economies with naïve agents and economies with sophisticated agents and provides assumptions under which both types of equilibria exist. Surprisingly, the set of naïve equilibria in societies populated by time-consistent households is not allocationally equivalent to the set of competitive equilibria. For sophisticated equilibria the equivalence holds.

Time-inconsistency also raises conceptual issues about the appropriate concept of efficiency. Choices have to be made concerning the incorporation of future preferences and the appropriate instruments to create Pareto improvements. For both naïve and sophisticated societies, we present four possible efficiency concepts. Suitable conditions are specified for which both naïve and sophisticated equilibria satisfy appropriate efficiency concepts.

\subsection{Introduction}

The vast majority of the economic literature assumes that preferences are timeconsistent. With time-consistent preferences a decision concerning a future date can be made at any period before that date and will not have to be reconsidered. Psychological research, however, has suggested that observed behavior is of-

\footnotetext{
${ }^{1}$ This chapter is based on Herings and Rohde (2006).
} 
ten time-inconsistent. Households frequently have intertemporal preferences that change over time. An example is a phenomenon known as hyperbolic discounting.

Under discounted utility a consumption stream is evaluated by first determining the value of consumption in each period would that consumption have taken place today, then multiplying each value by the discount factor corresponding to the period of consumption, and finally adding all these discounted values. With exponential discounting the discount factor is given by $\delta^{t}$, where $\delta$ is a constant and $t$ is the period of consumption. The hyperbolic discount function is given by $(1+\alpha t)^{-\beta / \alpha}$, where $\alpha, \beta>0$ (Loewenstein and Prelec, 1992). The quasi-hyperbolic discount factor is equal to 1 for $t=0$ and to $\beta \delta^{t}$ for $t>0$, where $0<\delta<1$ and $0<\beta<1$ (Phelps and Pollak, 1968). Thus, under exponential discounting the discount rate is constant, under quasi-hyperbolic discounting it is higher today than tomorrow, and constant from tomorrow on, and under hyperbolic discounting it is decreasing over time.

There is an extensive body of literature that claims that people tend to be more patient in the long run than in the short run, i.e. that discounting is not exponential. If discounting is not exponential preferences can be time-inconsistent. While a person may prefer one apple today to two apples tomorrow at any point in time, he might prefer two apples eleven days from the current period to one apple ten days from that period. Hyperbolic discounting can explain this phenomenon while maintaining the assumption of constant instantaneous preferences, but exponential discounting cannot. For evidence of time-inconsistent behavior, we refer to Thaler (1981), Ainslie and Haslam (1992), Loewenstein and Prelec (1992), Rachlin and Raineri (1992), and Frederick, Loewenstein and O'Donoghue (2002).

This chapter introduces time-inconsistent preferences in a multicommodity general equilibrium framework with incomplete markets. We take a general perspective on time-inconsistent preferences, which incorporates hyperbolic and quasihyperbolic discounting as special cases. We model households as consisting of a different self in every period. Thus, no intrapersonal conflicts can arise when only one period is studied. This perspective differs from the one in Benhabib and Bisin (2005), who assume that at every period a household has two conflicting preferences.

The contribution of this chapter is on a conceptual level. The introduction of time-inconsistent preferences in general equilibrium models requires a reformulation of concepts and definitions of behavior, equilibrium, and efficiency. The question of how to reformulate these concepts, is not trivial. It turns out that the introduction of time-inconsistent preferences makes the analysis quite complex. To 
alleviate the arising complications, and to highlight the conceptual issues, we restrict the analysis to the simplest market structure we can think of. In particular, we refrain from income transfers between periods.

We distinguish two types of societies, naïve and sophisticated ones. Naïve societies are populated by naïve households. These households do not realize that their intertemporal preferences change over time. Sophisticated societies consist of sophisticated households. A sophisticated household does realize that his intertemporal preferences will change in the future, and makes decisions today while anticipating these changes. We introduce the notions of naïve and sophisticated equilibrium as an extension of the usual notion of competitive equilibrium as applying to the time-consistent case. Surprisingly, the set of naïve equilibria of societies populated by time-consistent households is not allocationally equivalent to the set of competitive equilibria. For sophisticated equilibria, the equivalence holds. We give appropriate conditions under which both types of equilibria exist.

When intertemporal preferences change over time, the very definition of efficiency has to be reconsidered. Choices have to be made concerning the incorporation of future preferences and the appropriate instruments to create Pareto improvements. When efficiency is modeled as a program carried out by a social planner with certain objectives and instruments, we make a distinction between myopic and forward-looking social planners. We also distinguish planners who can change both actual and planned consumption and planners who can only change the former. For both naïve and sophisticated societies, this results in four possible efficiency concepts.

Related work on time-inconsistency in a general equilibrium setting has been done by Luttmer and Mariotti (2003, 2006), who study an infinite-horizon onegood model of an economy subject to uncertainty. Another related paper is the one of Krusell, Kuruşçu, and Smith (2002), who study an infinite horizon one-good model of a representative-agent economy without uncertainty. We will show how the efficiency concepts presented in those papers relate to our more general set-up.

The outline of this chapter is as follows. Section 5.2 introduces the model. The definition and existence of competitive equilibria in naïve societies is the subject of Section 5.3. The definition of equilibrium and the proof of its existence for sophisticated economies is analyzed in Section 5.4. Sections 5.5 and 5.6 introduce the appropriate concepts of constrained optimality, and discuss them in relation to naïve and sophisticated economies. Section 5.5 considers myopic social planners, while Section 5.6 considers forward-looking social planners. Finally, Section 5.7 concludes. Proofs are in the Appendix. 


\subsection{The Model}

Vector inequalities will be denoted by $\gg,>, \geq, \leq,<$, and $\ll$. We study a multiperiod multi-commodity general equilibrium model with incomplete markets that is not subject to uncertainty. There are $T$ periods that are indexed by $t \in T$. In each period, the exchange economy consists of $H$ households, indexed by $h \in H$, and $L$ commodities, indexed by $l \in L .^{2}$

With respect to periods, a distinction should be made between a planning period and a consumption period. At planning period $t$, plans are made for consumption in periods $\tau \geq t$.

At planning period 1, households expect to have a consumption set $X_{\cdot \mid 1}^{h} \subset$ $\mathbb{R}^{L T}$ for the remaining $T$ periods. It is assumed that households have correct expectations about their future consumption sets. This assumption implies that the consumption set at a planning period $t$ follows from the consumption plan realized so far and the consumption set $X_{\cdot \mid 1}^{h}$. Throughout the chapter, we assume that the consumption sets are independent of past consumption. ${ }^{3}$ Moreover, we will assume that $X_{\cdot \mid 1}^{h}=\mathbb{R}_{+}^{L T}$.

At every planning period $t$, households foresee an initial endowment $e_{\tau \mid t}^{h} \in \mathbb{R}^{L}$ for period $\tau$. Here again, households are assumed to have correct expectations, so $e_{\tau \mid t}^{h}$ is independent of the planning period $t$. At planning period $t$, the vector of all expected future endowments for household $h$ is represented by $e_{\cdot \mid t}^{h}=\left(e_{t \mid t}^{h}, \ldots, e_{T \mid t}^{h}\right)$.

At every planning period $t$, every household $h$ makes a consumption plan, which indicates how much it plans to consume in the current and future periods. For household $h$ the consumption in period $\tau$, as anticipated or planned in period $t$, is denoted by $x_{\tau \mid t}^{h} \in \mathbb{R}^{L}$. The planned consumption path for household $h$ at period $t$ is denoted by $x_{\cdot \mid t}^{h}=\left(x_{t \mid t}^{h}, \ldots, x_{T \mid t}^{h}\right)$. For practical purposes some other notation will be used: $x_{-\mid t}^{h}=\left(x_{1 \mid 1}^{h}, \ldots, x_{t-1 \mid t-1}^{h}\right)$ equals actual consumption up to period $t, x_{\tau, \tau^{\prime} \mid t}^{h}=\left(x_{\tau \mid t}^{h}, \ldots, x_{\tau^{\prime} \mid t}^{h}\right)$ is consumption planned at period $t$ for the periods $\tau$ up to $\tau^{\prime}$, and $x^{h}=\left(x_{\cdot \mid 1}^{h}, \ldots, x_{\cdot \mid T}^{h}\right)$ denotes a consumption bundle, i.e. $T$ consumption paths, of household $h$. When we drop the superscript $h$, the $H$-tuple over all households is taken, for instance $x_{\cdot \mid t}=\left(x_{\cdot \mid t}^{1}, \ldots, x_{\cdot \mid t}^{H}\right)$. Similarly, if we drop

\footnotetext{
${ }^{2}$ Notice that $T$ indicates both the number of time periods, and the set of time periods. Similarly, $H(L)$ indicates both the number of households (commodities) and the set of households (commodities). The context in which the symbol is used will make sure that no confusion can arise.

${ }^{3}$ Making the consumption sets depend on past consumption complicates the proofs of existence of equilibria. For instance, even when the endowments are in the interior of $X_{. \mid 1}^{h}$, for certain realized consumption plans they might be on the boundary of the consumption set at a future planning period.
} 
the subscript $t$, the $T$-tuple over all time periods is considered, $x=\left(x_{\cdot \mid 1}, \ldots, x_{\cdot \mid T}\right)$. For all the preceding vectors, a subscript $l$ is added if attention is restricted to a particular commodity $l$. We define the set $X_{-\mid t}^{h}=\mathbb{R}_{+}^{L(t-1)}$. The sets $X_{\tau \mid t}^{h}, X_{\cdot \mid t}^{h}$, $X_{\tau, \tau^{\prime} \mid t}^{h}, X^{h}, X_{\tau \mid t}, X_{\cdot \mid t}$, and $X$ are defined by taking the appropriate projections and Cartesian products. In particular, we define $X_{\tau \mid t}^{h}=\mathbb{R}_{+}^{L}, X_{\cdot \mid t}^{h}=\mathbb{R}_{+}^{L(T-t+1)}$, $X_{\tau, \tau^{\prime} \mid t}^{h}=\mathbb{R}_{+}^{L\left(\tau^{\prime}-\tau+1\right)}, X^{h}=\mathbb{R}_{+}^{L T(T+1) / 2}, X_{\cdot \mid t}=\prod_{h \in H} X_{\cdot \mid t}^{h}$, and $X=\prod_{h \in H} X^{h}$.

We call $x \in X$ an allocation. Notice that $x$ consists of $T$ consumption paths for the entire economy, each one starting at a different time period. Consumption paths starting at different time periods are not necessarily consistent. We explicitly allow for the possibility that $x_{\tau \mid t} \neq x_{\tau \mid t^{\prime}}$. An allocation is called time-consistent if at all periods the same consumption is planned for a given future period. This is formally expressed by the following definition.

\section{Definition 5.1 Time-consistent Allocation}

An allocation $x$ is time-consistent if, for every $h \in H$, for every $t \in T$ we have $x_{\cdot \mid t}^{h}=x_{t, T \mid 1}^{h}$.

At every planning period, every household has preferences over present and future consumption bundles. These preferences may depend on consumption in the past. Preferences of household $h$ at planning period $t$, given past consumption $x_{-\mid t}^{h}$, are represented by the preference relation $\succeq_{x_{-\mid t}^{h}}^{h, t}$ defined on $X_{\cdot \mid t}^{h} \times X_{\cdot \mid t}^{h}$. With slight abuse of notation we will often write $\succeq_{x_{-\mid t}^{h}}$ instead of $\succeq_{x_{-\mid t}^{h}}^{h, t}$. When past consumption is clear from the context, it is sometimes omitted from the notation, and the preferences of household $h$ at planning period $t$ are denoted by $\succeq^{h, t}$. The preference $\succeq^{h}$ of household $h$ is the collection of preferences at all possible planning periods, contingent on all possible historical consumption paths, $\succeq^{h}=$ $\left(\succeq_{x_{-\mid t}^{h}}\right)_{t \in T, x_{-\mid t}^{h} \in X_{-\mid t}^{h}}$.

An economy is described by its primitives, being consumption sets, preferences, and endowments: $\mathcal{E}=\left(X^{h}, \succeq^{h}, e^{h}\right)_{h \in H}$.

Consider two consumption paths that coincide up to period $t^{\prime}>t$. Preferences of a household are said to be time-consistent if the household prefers one consumption path over the other at period $t^{\prime}$ if and only if it does so at period $t$.

\section{Definition 5.2 Time-consistent Preferences}

Preferences of household $h$ are time-consistent if for all periods $t, t^{\prime} \in T$ with $t<t^{\prime}$, for every $x_{-\mid t}^{h} \in X_{-\mid t}^{h}$, and $x_{\cdot \mid t}^{h}, \bar{x}_{\cdot \mid t}^{h} \in X_{\cdot \mid t}^{h}$ with $x_{t, t^{\prime}-1 \mid t}^{h}=\bar{x}_{t, t^{\prime}-1 \mid t}^{h}$ we have

$$
x_{\cdot \mid t}^{h} \succeq_{x_{-\mid t}^{h}}^{h, t} \bar{x}_{\cdot \mid t}^{h} \text { if and only if } x_{t^{\prime}, T \mid t}^{h} \succeq_{\left(x_{-\mid t}^{h}, x_{t, t^{\prime}-1 \mid t}^{h}\right)}^{h, t^{\prime}} \bar{x}_{t^{\prime}, T \mid t}^{h} .
$$


Preferences are said to be time-inconsistent if they are not time-consistent ${ }^{4}$.

The following lemma shows that for the verification of time-consistency of preferences it suffices to make only comparisons involving period 1 and period $t$. The proofs of all lemmas and theorems are in the appendix.

Lemma 5.3 If the preferences of household $h$ are such that for every $t \in T$, for every $x_{\cdot \mid 1}^{h}, \bar{x}_{\cdot \mid 1}^{h} \in X_{\cdot \mid 1}^{h}$ with $x_{1, t-1 \mid 1}^{h}=\bar{x}_{1, t-1 \mid 1}^{h}$,

$$
x_{\cdot \mid 1}^{h} \succeq^{h, 1} \bar{x}_{\cdot \mid 1}^{h} \text { if and only if } x_{t, T \mid 1}^{h} \succeq_{x_{1, t-1 \mid 1}^{h, t}}^{h} \bar{x}_{t, T \mid 1}^{h},
$$

then the preferences of household $h$ are time-consistent.

One of the implications of the lemma is that knowledge of the preference relation $\succeq^{h, 1}$, together with the requirement of time-consistency, is sufficient for the derivation of all preference relations $\succeq^{h, t}$.

The consumption paths chosen by the households depend on current and expected future prices. In period $t$, the expected prices for period $\tau$ are denoted by $p_{\tau \mid t} \in P_{\tau \mid t}=\mathbb{R}^{L}$. As before, the vector of expected prices, at planning period $t$, for present and future periods is denoted by $p_{\cdot \mid t}=\left(p_{t \mid t}, \ldots, p_{T \mid t}\right)$. The set of admissible price systems $P_{. \mid t}$ is defined accordingly. The expected prices, at planning period $t$, for periods $\tau$ up to $\tau^{\prime}$ are denoted by $p_{\tau, \tau^{\prime} \mid t}=\left(p_{\tau \mid t}, \ldots, p_{\tau^{\prime} \mid t}\right)$, and the complete price system over all periods is represented by $p=\left(p_{\cdot \mid 1}, \ldots, p_{\cdot \mid T}\right)$, where $P$ is defined appropriately. Finally, realized prices up to period $t$ are represented by $p_{-\mid t}=\left(p_{1 \mid 1}, \ldots, p_{t-1 \mid t-1}\right)$.

We follow Pollak (1968), in distinguishing between naïve and sophisticated households. Naïve households are not aware of their changing preferences. They do not realize that in the future they might be willing to reconsider choices made today. Thus, when making a consumption decision in planning period $t$, a naïve household $h$ only takes into account the prevailing preferences at that particular period, $\succeq_{x_{-\mid t}^{h}}$. Sophisticated households, on the other hand, are aware of their changing preferences and will take them into account when making current decisions. They will only consider future plans that they expect to stick to. That is, when planning future consumption in period $t$, they incorporate $\succeq_{x_{-\mid \tau}^{h}}$ for all $\tau \geq t$.

First, the behavior of naïve households is addressed. Demand and supply of commodities is identified and the existence of an equilibrium is established. An

\footnotetext{
${ }^{4}$ Note that we allow for a more general set of preferences than Laibson $(1997,1998)$, Angeletos et al. (2001) and Luttmer and Mariotti (2003, 2006), since we allow for preferences that are not time-separable.
} 
example illustrates the intuition behind the model. The following assumptions will be made throughout the chapter:

Ass. 1 For every $h \in H$, for every $t \in T$, the consumption set $X_{\cdot \mid t}^{h}=\mathbb{R}_{+}^{L(T-t+1)}$.

Ass. 2 For every $h \in H, t \in T$, and $x_{-\mid t}^{h} \in X_{-\mid t}^{h}$, the preference relation $\succeq_{x_{-\mid t}^{h}}$ is complete, transitive, and continuous on $X_{\cdot \mid t}^{h} \times X_{\cdot \mid t}^{h}$.

Ass. 3 For every $h \in H, t \in T$, and $x_{-\mid t}^{h} \in X_{-\mid t}^{h}$, the preference relation $\succeq_{x_{-\mid t}^{h}}$ is monotone, i.e. for $x_{\cdot \mid t}^{h}, \bar{x}_{\cdot \mid t}^{h} \in X_{\cdot \mid t}^{h}$ with $\bar{x}_{\cdot \mid t}^{h} \geq x_{\cdot \mid t}^{h}$ and $\bar{x}_{\tau \mid t}^{h} \gg x_{\tau \mid t}^{h}$ for some $\tau \geq t$, we have $\bar{x}_{\cdot \mid t}^{h} \succ_{x_{-\mid t}^{h}} x_{\cdot \mid t}^{h}$.

Ass. 4 For every $h \in H, t \in T$, and $x_{-\mid t}^{h} \in X_{-\mid t}^{h}$, the preference relation $\succeq_{x_{-\mid t}^{h}}$ is convex in present and future consumption, i.e. for $x_{\cdot \mid t}^{h}, \bar{x}_{\cdot \mid t}^{h} \in X_{\cdot \mid t}^{h}$ with $\bar{x}_{\cdot \mid t}^{h} \succ_{x_{-\mid t}^{h}} x_{\cdot \mid t}^{h}$ we have $\alpha \bar{x}_{\cdot \mid t}^{h}+(1-\alpha) x_{\cdot \mid t}^{h} \succ_{x_{-\mid t}^{h}} x_{\cdot \mid t}^{h}$ for any $\alpha \in(0,1)$.

Ass. 5 For every $h \in H, e_{\cdot \mid 1}^{h} \gg 0$.

A preference relation $\succeq$ is continuous on $X \times X$ if for all $x \in X$ we have that $\{y \in$ $X: y \succeq x\}$ and $\{y \in X: y \preceq x\}$ are closed in $X$. The completeness, transitivity and continuity assumptions on preferences ensure that there are continuous utility functions $u_{x_{-\mid t}^{h}}$ representing the preferences.

\subsection{Naïve Societies}

This section considers naïve households. We treat the most simple incomplete markets case, where links between periods result from intertemporal preferences only. In planning period $t$, given a price vector $p_{\text {. } \mid t}$, the naïve household will have to make sure that in each future period the value of its consumption bundle in that period does not exceed the value of its endowment. That is, the opportunity set of the naïve household $h$ at period $t$ is defined by

$$
\gamma_{t}^{h}\left(p_{\cdot \mid t}\right)=\left\{x_{\cdot \mid t}^{h} \in X_{\cdot \mid t}^{h} \mid p_{\tau \mid t} x_{\tau \mid t}^{h} \leq p_{\tau \mid t} e_{\tau \mid t}^{h} \text { for all } \tau \geq t\right\}
$$

The demand set of household $h$ at period $t$ is then given by

$$
\delta_{t}^{h}\left(p_{\cdot \mid t}, x_{-\mid t}^{h}\right)=\left\{\tilde{x}_{\cdot \mid t}^{h} \in \gamma_{t}^{h}\left(p_{\cdot \mid t}\right) \mid \tilde{x}_{\cdot \mid t}^{h} \succeq_{x_{-\mid t}^{h}} x_{\cdot \mid t}^{h} \text { for all } x_{\cdot \mid t}^{h} \in \gamma_{t}^{h}\left(p_{\cdot \mid t}\right)\right\} .
$$

In a standard competitive analysis, preferences are implicitly assumed to be timeconsistent. In our more general setting, one could define a competitive equilibrium as follows. 


\section{Definition 5.4 Competitive Equilibrium}

A pair $\left(p_{\cdot \mid 1}^{*}, x_{\cdot \mid 1}^{*}\right) \in P_{\cdot \mid 1} \times X_{\cdot \mid 1}$ is a competitive equilibrium of the economy $\mathcal{E}$ if

(a) $x_{\cdot \mid 1}^{* h} \in \delta_{1}^{h}\left(p_{\cdot \mid 1}^{*}\right)$ for all $h \in H$,

(b) $\sum_{h \in H} x_{\cdot \mid 1}^{* h}=\sum_{h \in H} e_{\cdot \mid 1}^{h}$.

This definition only concerns the behavior in the first period. Obviously, this makes sense only if preferences are time-consistent. Another implicit assumption in the definition of competitive equilibrium in the standard setting is that allocations are time-consistent, as well as expectations of future prices. This observation leads to the following concept of extended competitive equilibrium.

\section{Definition 5.5 Extended Competitive Equilibrium}

A pair $\left(p^{*}, x^{*}\right) \in P \times X$ is an extended competitive equilibrium of the economy $\mathcal{E}$ if

(a) $\left(p_{\cdot \mid 1}^{*}, x_{\cdot \mid 1}^{*}\right)$ is a competitive equilibrium,

(b) $p_{\cdot \mid t}^{*}=p_{t, T \mid 1}^{*}$ for every $t \in T$, and

(c) $x_{\cdot \mid t}^{* h}=x_{t, T \mid 1}^{* h}$ for every $h \in H$ and every $t \in T$.

To define a competitive equilibrium that is appropriate for the study of economies with time-inconsistent preferences, we first assume that all households are naïve and maximize their utilities given past consumption. Thus, at any given price system, every household demands a future consumption path that is in its demand set. The price system and demanded consumption bundles will constitute an equilibrium if at any planning period, for every commodity, the total demand for that commodity does not exceed the total endowment of that commodity. Since preferences can be time-inconsistent, it may well be that the planned consumption bundles and prices will not be equal to the actual consumption bundles and prices. However, naïve households are not able to foresee their changing preferences and the resulting changing consumption bundles and prices. Thus, at an equilibrium price system there is no household that wants to deviate at any period from the consumption plan at that period, given the prices and price expectations at that period. This leads to the following definition of an equilibrium for naïve households.

\section{Definition 5.6 Naïve Equilibrium}

A pair $\left(p^{*}, x^{*}\right) \in P \times X$ is a naïve equilibrium of the economy $\mathcal{E}$ if

(a) $x_{\cdot \mid t}^{* h} \in \delta_{t}^{h}\left(p_{\cdot \mid t}^{*}, x_{-\mid t}^{* h}\right)$ for all $h \in H$ and all $t \in T$,

(b) $\sum_{h \in H} x_{\cdot \mid t}^{* h}=\sum_{h \in H} e_{\cdot \mid t}^{h}$ for all $t \in T$. 
The following theorem claims that the set of extended competitive equilibria is a subset of the set of naïve equilibria if preferences are time-consistent.

Theorem 5.7 If preferences of all households are time-consistent, then an extended competitive equilibrium of the economy $\mathcal{E}$ is a naïve equilibrium.

The following example shows that the converse is not necessarily true. Even if preferences of all households are time-consistent, a naïve equilibrium of the economy might not be an extended competitive equilibrium. Notice that in a naïve equilibrium all price expectations are correct and consistent with market clearing in all periods. Our two-periods example is constructed in such a way that at the beginning of the second period, two equilibrium continuations are possible. Since preferences are time-consistent, one of these equilibrium continuations yields an extended competitive equilibrium. The other equilibrium continuation, however, turns out not to be consistent with any extended competitive equilibrium, but does yield a naïve equilibrium.

\section{Example 5.8}

Consider an economy with two naïve households, two goods and two periods. The endowments of the households are $e_{\cdot \mid 1}^{1}=\left(e_{1 \mid 1}^{1}, e_{2 \mid 1}^{1}\right)=(1,2,0,4)$ and $e_{\cdot \mid 1}^{2}=$ $\left(e_{1 \mid 1}^{2}, e_{2 \mid 1}^{2}\right)=(2,1,4,0)$. The time-consistent preferences are given by

$$
u^{1}\left(x_{1 \mid 1}^{1}, x_{2 \mid 1}^{1}\right)=\left\{\begin{array}{r}
\min \left(x_{1,1 \mid 1}^{1}, x_{1,2 \mid 1}^{1}, x_{2,1 \mid 1}^{1}, x_{2,2 \mid 1}^{1}\right) \\
\quad \text { if } \min \left(x_{1,1 \mid 1}^{1}, x_{1,2 \mid 1}^{1}, x_{2,1 \mid 1}^{1}, x_{2,2 \mid 1}^{1}\right) \leq 1 \\
{\left[\left(x_{1,1 \mid 1}^{1}-1\right)\left(x_{1,2 \mid 1}^{1}-1\right)\left(x_{2,1 \mid 1}^{1}-1\right)\left(x_{2,2 \mid 1}^{1}-1\right)\right]^{1 / 4}+1} \\
\text { if } \min \left(x_{1,1 \mid 1}^{1}, x_{1,2 \mid 1}^{1}, x_{2,1 \mid 1}^{1}, x_{2,2 \mid 1}^{1}\right) \geq 1
\end{array}\right.
$$

for household 1 and

$$
u^{2}\left(x_{1 \mid 1}^{2}, x_{2 \mid 1}^{2}\right)=\min \left(x_{1,1 \mid 1}^{2}, x_{1,2 \mid 1}^{2}, x_{2,1 \mid 1}^{2}, x_{2,2 \mid 1}^{2}\right)
$$

for household 2 .

Consider prices $p^{*}$ such that $p_{\cdot \mid 1}^{*}=(1,2,4,1)$ and $p_{\cdot \mid 2}^{*}=(3,4)$. Then for household 1 we have $\min \left(x_{2,1 \mid 1}^{1}, x_{2,2 \mid 1}^{1}\right) \leq 4 / 5<1$. Thus, $x_{\cdot \mid 1}^{* 1}=\left(1 \frac{2}{3}, 1 \frac{2}{3}, \frac{4}{5}, \frac{4}{5}\right)$ is an optimal consumption bundle for household 1. Moreover, $x_{\cdot \mid 1}^{* 2}=\left(1 \frac{1}{3}, 1 \frac{1}{3}, \frac{16}{5}, \frac{16}{5}\right)$ is an optimal consumption bundle for household 2. By time-consistency of preferences, when arriving in the second period, the households maximize the following 
utility functions

$$
u^{1}\left(x_{1 \mid 1}^{* 1}, x_{2 \mid 2}^{1}\right)=\left\{\begin{array}{l}
\min \left(1 \frac{2}{3}, 1 \frac{2}{3}, x_{2,1 \mid 2}^{1}, x_{2,2 \mid 2}^{1}\right) \\
\text { if } \min \left(x_{2,1 \mid 2}^{1}, x_{2,2 \mid 2}^{1}\right) \leq 1 \\
{\left[\frac{2}{3} \cdot \frac{2}{3} \cdot\left(x_{2,1 \mid 2}^{1}-1\right)\left(x_{2,2 \mid 2}^{1}-1\right)\right]^{1 / 4}+1} \\
\text { if } \min \left(x_{2,1 \mid 2}^{1}, x_{2,2 \mid 2}^{1}\right) \geq 1
\end{array}\right.
$$

and

$$
u^{2}\left(x_{1 \mid 1}^{* 2}, x_{2 \mid 2}^{2}\right)=\min \left(1 \frac{1}{3}, 1 \frac{1}{3}, x_{2,1 \mid 2}^{2}, x_{2,2 \mid 2}^{2}\right)
$$

With prices $p_{\cdot \mid 2}^{*}$, the second-period budget constraint for household 1 implies that $x_{2,2 \mid 2}^{1}=4-3 x_{2,1 \mid 2}^{1} / 4$. The first household then maximizes $\left(x_{2,1 \mid 2}^{1}-1\right)\left(x_{2,2 \mid 2}^{1}-1\right)$ subject to that budget constraint, which yields $x_{2 \mid 2}^{* 1}=\left(2 \frac{1}{2}, 2 \frac{1}{8}\right)$. For household $2, x_{2 \mid 2}^{* 2}=\left(1 \frac{1}{2}, 1 \frac{7}{8}\right)$ is an optimal consumption bundle. Thus, $\left(p^{*}, x^{*}\right)$ is a naïve equilibrium.

The allocation $\left(\left(x_{1 \mid 1}^{* 1}, x_{2 \mid 2}^{* 1}\right),\left(x_{1 \mid 1}^{* 2}, x_{2 \mid 2}^{* 2}\right)\right)=\left(\left(1 \frac{2}{3}, 1 \frac{2}{3}, 2 \frac{1}{2}, 2 \frac{1}{8}\right),\left(1 \frac{1}{3}, 1 \frac{1}{3}, 1 \frac{1}{2}, 1 \frac{7}{8}\right)\right)$ cannot be a competitive equilibrium allocation. Suppose to the contrary that this allocation is a competitive equilibrium allocation. Since household 1 demands more than one unit of each good for the second period, it maximizes $\left(x_{1,1 \mid 1}^{1}-1\right)\left(x_{1,2 \mid 1}^{1}-1\right)$ in the first period subject to the budget constraint. By deriving the first-order conditions of that problem, it can easily be seen that household 1 will demand an equal amount of both goods in the first period only if $p_{1,1 \mid 1}=p_{1,2 \mid 1}$. But then again, it would demand $1 \frac{1}{2}$ units of each good in the first period, instead of $1 \frac{2}{3}$ units. Thus, we arrive at a contradiction. This shows that $\left(\left(x_{1 \mid 1}^{* 1}, x_{2 \mid 2}^{* 1}\right),\left(x_{1 \mid 1}^{* 2}, x_{2 \mid 2}^{* 2}\right)\right)$ cannot be a competitive equilibrium allocation. By similar arguments, $p=(1,2,3,4)$ cannot be a competitive equilibrium price system.

Although a naïve equilibrium allocation might be incompatible with any extended competitive equilibrium, a weaker result can be obtained. If preferences are time-consistent and a naïve equilibrium exists, then at least one of the naïve equilibria is an extended competitive equilibrium as well. This can be derived from the next theorem combined with Theorem 5.7.

Theorem 5.9 Assume that preferences are time-consistent. If a naïve equilibrium exists in the economy $\mathcal{E}$, then also an extended competitive equilibrium exists.

A naïve equilibrium can be shown to exist under standard assumptions.

Theorem 5.10 (Existence of naïve equilibrium) If the economy $\mathcal{E}$ satisfies Assumptions 1-5, then there exists a naïve equilibrium $\left(p^{*}, x^{*}\right)$. 
The proof of the theorem requires an induction argument. That is, we first establish the existence of equilibrium prices and allocations as planned in the first period. Then given the first period equilibrium, we show the existence of equilibrium prices and allocations as planned in the second period, and so on.

\subsection{Sophisticated Societies}

This section considers sophisticated households. Again, we treat the most simple incomplete markets case, where links between periods result from intertemporal preferences only. The introduction of sophisticated households gives rise to new phenomena. The difference between a naïve and a sophisticated household is that the former is not aware of its changing preferences, whereas the latter is. A sophisticated household will only make consumption plans for the future that it expects to actually stick to. A sophisticated household can be seen as consisting of different selves, where the first self acts first and the next selves act subsequently. The behavior of the household can then be modeled as a game where the players are the different selves. A sophisticated household will only play a subgame-perfect Nash equilibrium of that game.

In the last period, no plans for the future are made. Thus, in the last period, the opportunity and demand sets of the sophisticated households resemble those for the naïve households. More specifically, the opportunity set in the last period is defined by

$$
\phi_{T}^{h}\left(p_{\cdot \mid T}, x_{-\mid T}^{h}\right)=\left\{x_{\cdot \mid T}^{h} \in X_{\cdot \mid T}^{h} \quad \mid p_{T \mid T} x_{T \mid T}^{h} \leq p_{T \mid T} e_{T \mid T}^{h}\right\} .
$$

The set of optimal consumption bundles in the last period is given by

$$
\begin{aligned}
& \xi_{T}^{h}\left(p_{\cdot \mid T}, x_{-\mid T}^{h}\right)=\left\{\tilde{x}_{\cdot \mid T}^{h} \in \phi_{T}^{h}\left(p_{\cdot \mid T}, x_{-\mid T}^{h}\right) \mid\right. \\
&\left.\tilde{x}_{\cdot \mid T}^{h} \succeq_{x_{-\mid T}^{h}} x_{\cdot \mid T}^{h} \text { for all } x_{\cdot \mid T}^{h} \in \phi_{T}^{h}\left(p_{\cdot \mid T}, x_{-\mid T}^{h}\right)\right\} .
\end{aligned}
$$

The opportunity sets in earlier periods are similar to those for the naïve households, except for the fact that the sophisticated household restricts himself to future consumption plans that are in his future demand sets at the expected future prices. That is, the opportunity set for the sophisticated household $h$ in period $t, t<T$, is defined by

$$
\begin{aligned}
\phi_{t}^{h}\left(p_{\cdot \mid t}, x_{-\mid t}^{h}\right)=\left\{x_{\cdot \mid t}^{h} \in X_{\cdot \mid t}^{h} \mid p_{\tau \mid t} x_{\tau \mid t}^{h} \leq p_{\tau \mid t} e_{\tau \mid t}^{h} \text { for all } \tau \geq t, \quad\right. \text { and } \\
\left.\qquad x_{t+1, T \mid t}^{h} \in \xi_{t+1}^{h}\left(p_{t+1, T \mid t}, x_{-\mid t}^{h}, x_{t \mid t}^{h}\right)\right\} .
\end{aligned}
$$


Since preferences depend on past consumption, the opportunity sets also depend on past consumption. The demand set for household $h$ in period $t, t<T$, is then given by:

$$
\begin{aligned}
& \xi_{t}^{h}\left(p_{\cdot \mid t}, x_{-\mid t}^{h}\right)=\left\{\tilde{x}_{\cdot \mid t}^{h} \in \phi_{t}^{h}\left(p_{\cdot \mid t}, x_{-\mid t}^{h}\right) \mid\right. \\
&\left.\tilde{x}_{\cdot \mid t}^{h} \succeq_{x_{-\mid t}^{h}} x_{\cdot \mid t}^{h} \text { for all } x_{\cdot \mid t}^{h} \in \phi_{t}^{h}\left(p_{\cdot \mid t}, x_{-\mid t}^{h}\right)\right\} .
\end{aligned}
$$

We introduce the following equilibrium concept for sophisticated societies.

\section{Definition 5.11 Sophisticated Equilibrium}

A pair $\left(p^{*}, x^{*}\right) \in P \times X$ is a sophisticated equilibrium if

(a) $x_{\cdot \mid t}^{* h} \in \xi_{t}^{h}\left(p_{\cdot \mid t}^{*}, x_{-\mid t}^{* h}\right)$ for all $h \in H$ and all $t \in T$,

(b) $\sum_{h \in H} x_{\cdot \mid t}^{* h}=\sum_{h \in H} e_{\cdot \mid t}^{h}$ for all $t \in T$,

(c) $p_{t^{\prime}, T \mid t}^{*}=p_{\cdot \mid t^{\prime}}^{*}$ for all $t, t^{\prime} \in T$ with $t \leq t^{\prime}$,

(d) $x_{t^{\prime}, T \mid t}^{* h}=x_{\cdot \mid t^{\prime}}^{* h}$ for all $t, t^{\prime} \in T$ with $t \leq t^{\prime}$.

As sophisticated households make plans that they will stick to in the future, we follow Arrow (1953) and Radner (1972) and define an equilibrium price system in such a way that expected prices are equal to actual prices, i.e. that households have correct point expectations about future prices. ${ }^{5}$ Furthermore, it is also assumed that consumption choices will not have to be reconsidered.

The next theorem presents a characterization of the notion of sophisticated equilibrium.

Theorem 5.12 A pair $\left(p^{*}, x^{*}\right) \in P \times X$ is a sophisticated equilibrium if and only if it satisfies the following conditions:

(i) $x_{\cdot \mid 1}^{* h} \in \xi_{1}^{h}\left(p_{\cdot \mid 1}^{*}\right)$ for all $h \in H$,

(ii) $\sum_{h \in H} x_{\cdot \mid 1}^{* h}=\sum_{h \in H} e_{\cdot \mid 1}^{h}$ for all $h \in H$,

(iii) $p_{\cdot \mid t}^{*}=p_{t, T \mid 1}^{*}$ for all $t \in T$,

(iv) $x_{\cdot \mid t}^{* h}=x_{t, T \mid 1}^{* h}$ for all $h \in H$ and all $t \in T$.

The next result shows that if preferences are time-consistent, then the set of sophisticated equilibria coincides with the set of extended competitive equilibria.

\footnotetext{
${ }^{5}$ See Dutta and Morris (1997) for alternatives to the concept of rational expectations as used by Arrow (1953) and Radner (1972).
} 
Theorem 5.13 Assume that the preferences of all households are time-consistent and that Assumptions 1-2 hold. A pair $\left(p^{*}, x^{*}\right) \in P \times X$ with $p^{*} \gg 0$ is a sophisticated equilibrium of the economy $\mathcal{E}$ if and only if it is an extended competitive equilibrium.

We will establish the existence of a sophisticated equilibrium under some additional assumptions. Therefore, we first define independence of past consumption.

\section{Definition 5.14 Independence of past consumption}

Preferences are independent of past consumption when $\succeq_{x_{-\mid t}^{h}}=\succeq_{\bar{x}_{-\mid t}^{h}}$ for every $x_{-\mid t}^{h}, \bar{x}_{-\mid t}^{h} \in X_{-\mid t}^{h}$.

The additional assumptions are as follows:

Ass. 4' For every $h \in H, t \in T$, and $x_{-\mid t}^{h} \in X_{-\mid t}^{h}$, the preference relation $\succeq_{x_{-\mid t}^{h}}$ is strictly convex in present and future consumption, i.e. for $x_{\cdot \mid t}^{h}, \bar{x}_{\cdot \mid t}^{h} \in X_{\cdot \mid t}^{h}$ with $\bar{x}_{\cdot \mid t}^{h} \succeq_{x_{-\mid t}^{h}} x_{\cdot \mid t}^{h}$ and $\bar{x}_{\cdot \mid t}^{h} \neq x_{\cdot \mid t}^{h}$ we have $\alpha \bar{x}_{\cdot \mid t}^{h}+(1-\alpha) x_{\cdot \mid t}^{h} \succ_{x_{-\mid t}^{h}} x_{\cdot \mid t}^{h}$ for any $\alpha \in(0,1)$.

Ass. 6 Preferences are independent of past consumption.

Assumption 6 does not allow for habit formation, where consumption depends on consumption in the past. However, it does allow for intertemporal utility functions that discount hyperbolically or quasi-hyperbolically.

If the assumptions, and in particular Assumption 6, are not satisfied, it may well happen that an equilibrium does not exist. In that case, it cannot be guaranteed that demand correspondences are convex-valued. This is illustrated in the following example.

\section{Example 5.15}

Consider an economy with two sophisticated households $h \in\{1,2\}$, two goods $l \in\{1,2\}$ and two periods $t \in\{1,2\}$. The endowments of the first and the second household are respectively given by $e_{\cdot \mid 1}^{1}=(1,0,1,1)$ and $e_{\cdot \mid 1}^{2}=(0,1,1,0)$. Let the preferences of household 1 in respectively the first and the second period be

$$
\begin{aligned}
& u_{1}^{1}\left(x_{1 \mid 1}^{1}, x_{2 \mid 1}^{1}\right)=x_{1,1 \mid 1}^{1}+x_{2,1 \mid 1}^{1}+\frac{1}{2}\left(x_{1,2 \mid 1}^{1}+x_{2,2 \mid 1}^{1}\right) \\
& u_{2}^{1}\left(x_{1 \mid 1}^{1}, x_{2 \mid 2}^{1}\right)= \begin{cases}\frac{\ln x_{2,1 \mid 2}^{1}}{x_{1,1 \mid 1}^{1}}+\ln x_{2,2 \mid 2}^{1} & \text { if } x_{1,1 \mid 1}^{1}>1 / 3 \\
3 \ln x_{2,1 \mid 2}^{1}+\ln x_{2,2 \mid 2}^{1} & \text { otherwise. }\end{cases}
\end{aligned}
$$


Note that $u_{2}^{1}\left(x_{1 \mid 1}^{1}, x_{2 \mid 2}^{1}\right)$ is continuous in $x_{2,1 \mid 2}^{1}$ and $x_{2,2 \mid 2}^{1}$. Given second-period prices $p_{\cdot \mid 2}=p_{2 \mid 1} \gg 0$, the demand of household 1 for the second period satisfies

$$
\begin{aligned}
x_{2,1 \mid 2}^{1} & =\frac{p_{2,1 \mid 2}+p_{2,2 \mid 2}}{p_{2,1 \mid 2}} \frac{1}{x_{1,1 \mid 1}^{1}} \frac{1}{\frac{1}{x_{1,1 \mid 1}^{1}}+1}=\frac{p_{2,1 \mid 2}+p_{2,2 \mid 2}}{p_{2,1 \mid 2}} \frac{1}{1+x_{1,1 \mid 1}^{1}}, \\
x_{2,2 \mid 2}^{1} & =\frac{p_{2,1 \mid 2}+p_{2,2 \mid 2}}{p_{2,2 \mid 2}} \frac{x_{1,1 \mid 1}^{1}}{1+x_{1,1 \mid 1}^{1}}
\end{aligned}
$$

if $x_{1,1 \mid 1}^{1}>1 / 3$, and

$$
\begin{aligned}
& x_{2,1 \mid 2}^{1}=\frac{p_{2,1 \mid 2}+p_{2,2 \mid 2}}{p_{2,1 \mid 2}} \frac{1}{1+1 / 3}, \\
& x_{2,2 \mid 2}^{1,2}=\frac{p_{2,1 \mid 2}+p_{2,2 \mid 2}}{p_{2,2 \mid 2}} \frac{1 / 3}{1+1 / 3}
\end{aligned}
$$

if $x_{1,1 \mid 1}^{1} \leq 1 / 3$.

By substituting these second period demands and the first period budget constraint in the first period utility function, household 1 maximizes the indirect utility function $v$ in period 1 , where

$$
\begin{aligned}
v\left(x_{1,1 \mid 1}^{1}\right)= & x_{1,1 \mid 1}^{1}+\frac{p_{2,1 \mid 2}+p_{2,2 \mid 2}}{p_{2,1 \mid 2}} \frac{1}{1+x_{1,1 \mid 1}^{1}} \\
& +\frac{1}{2}\left(\frac{p_{1,1 \mid 1}-p_{1,1 \mid 1} x_{1,1 \mid 1}^{1}}{p_{1,2 \mid 1}}+\frac{p_{2,1 \mid 2}+p_{2,2 \mid 2}}{p_{2,2 \mid 2}} \frac{x_{1,1 \mid 1}^{1}}{1+x_{1,1 \mid 1}^{1}}\right) \\
= & \frac{p_{1,1 \mid 1}}{2 p_{1,2 \mid 1}}+\left(1-\frac{p_{1,1 \mid 1}}{2 p_{1,2 \mid 1}}\right) x_{1,1 \mid 1}^{1}+\frac{p_{2,1 \mid 2}+p_{2,2 \mid 2}}{1+x_{1,1 \mid 1}^{1}}\left(\frac{1}{p_{2,1 \mid 2}}+\frac{x_{1,1 \mid 1}^{1}}{2 p_{2,2 \mid 2}}\right)
\end{aligned}
$$

if $x_{1,1 \mid 1}^{1}>1 / 3$, and

$$
\begin{aligned}
v\left(x_{1,1 \mid 1}^{1}\right)= & x_{1,1 \mid 1}^{1}+\frac{p_{2,1 \mid 2}+p_{2,2 \mid 2}}{p_{2,1 \mid 2}} \frac{1}{1+1 / 3} \\
& +\frac{1}{2}\left(\frac{p_{1,1 \mid 1}-p_{1,1 \mid 1} x_{1,1 \mid 1}^{1}}{p_{1,2 \mid 1}}+\frac{p_{2,1 \mid 2}+p_{2,2 \mid 2}}{p_{2,2 \mid 2}} \frac{1 / 3}{1+1 / 3}\right)
\end{aligned}
$$

if $x_{1,1 \mid 1}^{1} \leq 1 / 3$. Note that in the former case $v$ is a nonlinear function, whereas in the latter case it is linear. The demand correspondence resulting from $v$ is not everywhere convex-valued. 
The time-consistent preferences of household 2 in respectively the first and the second period are given by

$$
\begin{aligned}
& u_{1}^{2}\left(x_{1 \mid 1}^{2}, x_{2 \mid 1}^{2}\right)=\min \left(x_{1,1 \mid 1}^{2}, x_{1,2 \mid 1}^{2}\right)+x_{2,1 \mid 2}^{2}+2 x_{2,2 \mid 2}^{2}, \\
& u_{2}^{2}\left(x_{1 \mid 1}^{2}, x_{2 \mid 2}^{2}\right)=x_{2,1 \mid 2}^{2}+2 x_{2,2 \mid 2}^{2} .
\end{aligned}
$$

In the appendix we show that there is no sophisticated equilibrium in this economy.

The following theorem shows that, with our assumption, sophisticated equilibria exist.

Theorem 5.16 Existence of sophisticated equilibrium If the economy $\mathcal{E}$ satisfies Assumptions 1-6 and 4', then there exists a sophisticated equilibrium $\left(p^{*}, x^{*}\right)$.

The existence proof is standard and can be found in the appendix. The major complication to be taken care of is the part of the proof that shows a sophisticated equilibrium of the compactified economy to remain an equilibrium after the bounds on consumption sets have been removed.

\subsection{Efficiency - the Myopic Case}

When intertemporal preferences change over time, the very definition of efficiency has to be reconsidered. Choices have to be made concerning the incorporation of future preferences in the efficiency notion used and the appropriate instruments allowed to create Pareto improvements. When efficiency is modeled as a program carried out by a social planner with certain objectives and instruments, we can make a distinction between myopic and forward-looking social planners. Myopic social planners care only about the current self of every household. Forwardlooking social planners take all selves of every household into account. As far as instruments are concerned, we distinguish between social planners who can modify both actual and planned consumption and social planners who can only change actual consumption. For the former type of social planner there exists a naïve and a sophisticated version, where the latter version sticks to time-consistent allocations. We will show that for the myopic case the latter distinction is immaterial.

This section considers social planners who care only about the current self of each household. When a social planner takes into account only the intertemporal preferences of the households in one particular period, this can mean that the 


\begin{tabular}{|c|c|c|c|c|}
\hline & \multicolumn{3}{|c|}{ Instruments } \\
\hline & & \multirow[t]{2}{*}{$\begin{array}{c}\text { Current } \\
\text { Consumption }\end{array}$} & \multicolumn{2}{|c|}{$\begin{array}{l}\text { Current and Planned } \\
\text { Consumption }\end{array}$} \\
\hline & & & $\begin{array}{c}\text { Naïve } \\
\text { Societies }\end{array}$ & $\begin{array}{l}\text { Sophisticated } \\
\text { Societies }\end{array}$ \\
\hline \multirow[t]{2}{*}{ Goals } & Myopic & $\begin{array}{c}\text { CMP } \\
\text { Section 5.5.2 }\end{array}$ & $\begin{array}{c}\text { MOP } \\
\text { Section 5.5.1 }\end{array}$ & $\begin{array}{c}\text { MOP } \\
\text { Section 5.5.1 }\end{array}$ \\
\hline & $\begin{array}{c}\text { Forward- } \\
\text { looking }\end{array}$ & $\begin{array}{c}\mathrm{CP} \\
\text { Section 5.6.2 }\end{array}$ & $\begin{array}{c}\text { OP } \\
\text { Section 5.6.1 }\end{array}$ & $\begin{array}{c}\text { TCOP } \\
\text { Section 5.6.1 }\end{array}$ \\
\hline
\end{tabular}

Table 5.1: Summary of efficiency concepts

social planner cares only about the selves corresponding to the period in which the planner is active and is myopic in that he forgets to realize that the preferences of future selves might differ from the ones of current selves. Another interpretation is that the social planner has reasons to believe that the preferences of the current selves of the households are the true underlying preferences of the households and that the preferences of the future selves of the households are distorted preferences.

\subsubsection{Myopic Overall Pareto Efficiency}

In this subsection we assume that social planners are myopic and can alter both actual and planned consumption. An allocation is called myopic overall Pareto efficient if there is no planning period $t$ where actual and planned consumption in that particular period could be reallocated in such a way that every household would be at least as well off in that period as at the original allocation, whereas one household would be strictly better off than at the original allocation.

\section{Definition 5.17 Myopic Overall Pareto (MOP) Efficiency}

The allocation $x^{*}$ is myopic overall Pareto (MOP) efficient if there is no allocation $\tilde{x}$ and no period $t^{\prime}$ such that

(i) $\sum_{h \in H} \tilde{x}_{\cdot \mid t^{\prime}}^{h}=\sum_{h \in H} e_{\cdot \mid t^{\prime}}^{h}$,

(ii) $\tilde{x}_{\cdot \mid t^{\prime}}^{h} \succeq_{x_{-\mid t^{\prime}}^{* h}} x_{\cdot \mid t^{\prime}}^{* h}$ for all $h \in H$, and

(iii) $\tilde{x}_{\cdot \mid t^{\prime}}^{h^{\prime}} \succ_{x_{-\mid t^{\prime}}^{* h^{\prime}}} x_{\cdot \mid t^{\prime}}^{* h^{\prime}}$ for some $h^{\prime} \in H$. 
The intuition behind this definition is as follows. In every planning period $t$ there is a social planner who seeks to maximize only the preferences of the selves of the households at period $t$. The social planner reallocates both current and planned consumption. Now an equilibrium is called MOP efficient if there is no sequence of social planners that behave as described and that can make at least one household better off than in equilibrium, while not making any household worse off. If preferences do not depend on past consumption this concept corresponds to "datet Pareto efficiency" for every $t$ as introduced in Luttmer and Mariotti (2006). In addition, a MOP efficient allocation is renegotiation-proof in the sense of Luttmer and Mariotti (2006).

MOP efficiency is closely related to unconstrained Pareto efficiency. In our multi-period context, by restricting attention to the preferences of households at period 1, Pareto efficiency could be defined as follows.

\section{Definition 5.18 Pareto Efficiency}

The allocation $x^{*}$ is Pareto efficient if there is no allocation $\tilde{x}$ such that

(i) $\sum_{h \in H} \tilde{x}_{\cdot \mid 1}^{h}=\sum_{h \in H} e_{\cdot \mid 1}^{h}$,

(ii) $\tilde{x}_{\cdot \mid 1}^{h} \succeq^{h, 1} x_{\cdot \mid 1}^{* h}$ for all $h \in H$, and

(iii) $\tilde{x}_{\cdot \mid 1}^{h^{\prime}} \succ^{h^{\prime}, 1} x_{\cdot \mid 1}^{* h^{\prime}}$ for some $h^{\prime} \in H$.

The following theorem shows that the two concepts are equivalent when preferences are time-consistent and when attention is restricted to time-consistent allocations.

Theorem 5.19 Assume that preferences are time-consistent. Then a time-consistent allocation is MOP efficient if and only if it is Pareto efficient.

In settings with incomplete markets, it has been shown that equilibria are typically not Pareto efficient. Generically, they are not even efficient when weaker efficiency concepts are used. ${ }^{6}$ Therefore, since we have a sequence of markets that do not allow for intertemporal income transfers, examples that show that naïve and sophisticated equilibria may be MOP inefficient can easily be found.

\section{Example 5.20}

Consider an economy with two households, two periods and two goods in each

\footnotetext{
${ }^{6}$ See for instance Geanakoplos and Polemarchakis (1986), Citanna, Kajii, and Villanacci (1998) and Herings and Polemarchakis (2005).
} 
period. Let the endowments be given by $e_{\cdot \mid \tau}^{h}=(1,1)$ for all $h, \tau$. The first period preferences of respectively the first and the second household are given by:

$$
\begin{aligned}
& u_{1}^{1}\left(x_{\cdot \mid 1}^{1}\right)=2\left(x_{1,1 \mid 1}^{1}+x_{1,2 \mid 1}^{1}\right)+x_{2,1 \mid 1}^{1}+x_{2,2 \mid 1}^{1} \\
& u_{1}^{2}\left(x_{\cdot \mid 1}^{2}\right)=x_{1,1 \mid 1}^{2}+x_{1,2 \mid 1}^{2}+2\left(x_{2,1 \mid 1}^{2}+x_{2,2 \mid 1}^{2}\right),
\end{aligned}
$$

and in the second period by

$$
\begin{aligned}
& u_{2}^{1}\left(x_{\cdot \mid 2}^{1}\right)=x_{2,1 \mid 2}^{1}+x_{2,2 \mid 2}^{1} \\
& u_{2}^{2}\left(x_{\cdot \mid 2}^{2}\right)=2\left(x_{2,1 \mid 2}^{2}+x_{2,2 \mid 2}^{2}\right) .
\end{aligned}
$$

Consider prices $p_{\tau \mid t}=(1,1)$ for every $\tau, t$ with $\tau \geq t$. Then the allocation

$$
\begin{aligned}
& \left(x_{1,1 \mid 1}^{* h}, x_{1,2 \mid 1}^{* h}, x_{2,1 \mid 1}^{* h}, x_{2,2 \mid 1}^{* h}\right)=(1,1,1,1), \\
& \left(x_{2,1 \mid 2}^{* h}, x_{2,2 \mid 2}^{* h}\right)=(1,1)
\end{aligned}
$$

is a naïve and sophisticated equilibrium. However, with the allocation $\tilde{x}$ as defined next, the households are better off in the first period.

$$
\begin{aligned}
& \left(\tilde{x}_{1,1 \mid 1}^{1}, \tilde{x}_{1,2 \mid 1}^{1}, \tilde{x}_{2,1 \mid 1}^{1}, \tilde{x}_{2,2 \mid 1}^{1}\right)=(2,2,0,0), \\
& \left(\tilde{x}_{1,1 \mid 1}^{2}, \tilde{x}_{1,2 \mid 1}^{2}, \tilde{x}_{2,1 \mid 1}^{2}, \tilde{x}_{2,2 \mid 1}^{2}\right)=(0,0,2,2), \\
& \left(\tilde{x}_{2,1 \mid 2}^{h}, \tilde{x}_{2,2 \mid 2}^{h}\right)=(1,1) .
\end{aligned}
$$

Thus, this naïve and sophisticated equilibrium is not MOP efficient.

The next example shows that a sophisticated equilibrium may be dominated by a naïve equilibrium. This result might be surprising. It contradicts the view that higher degrees of sophistication lead to higher utility.

\section{Example 5.21}

Consider a two-period economy with two households and two goods. Let $e_{\tau \mid 1}^{h}=$ $(1,1)$ for all $h, \tau$. The first-period utilities for the two households are as follows:

$$
\begin{aligned}
& u_{1}^{1}\left(x_{\cdot \mid 1}^{1}\right)=2\left(x_{1,1 \mid 1}^{1}+x_{2,1 \mid 1}^{1}\right)+x_{1,2 \mid 1}^{1}+x_{2,2 \mid 1}^{1}, \\
& u_{1}^{2}\left(x_{\cdot \mid 1}^{2}\right)=x_{1,1 \mid 1}^{2}+x_{2,1 \mid 1}^{2}+2\left(x_{1,2 \mid 1}^{2}+x_{2,2 \mid 1}^{2}\right),
\end{aligned}
$$

where the subscripts are of the form $\tau, l \mid t$. The utilities in the second period are given by:

$$
\begin{aligned}
& u_{2}^{1}\left(x_{2 \mid 2}^{1}\right)=x_{2,1 \mid 2}^{1}+2 x_{2,2 \mid 2}^{1}, \\
& u_{2}^{2}\left(x_{2 \mid 2}^{2}\right)=2 x_{2,1 \mid 2}^{2}+x_{2,2 \mid 2}^{2} .
\end{aligned}
$$


The utility functions are continuous and strictly increasing in every argument.

Suppose that the prices of the two goods are equal in the second period, i.e. $p_{2,1 \mid 2}=p_{2,2 \mid 2}$. Since household 1 (2) cares more about consumption of good 2 (1) in period 2 , it will spend all of its wealth on good 2 (1). More specifically, the demanded consumption bundles are as follows:

$$
\begin{aligned}
& \left(x_{2,1 \mid 2}^{* 1}, x_{2,2 \mid 2}^{* 1}\right)=(0,2), \\
& \left(x_{2,1 \mid 2}^{* 2}, x_{2,2 \mid 2}^{* 2}\right)=(2,0) .
\end{aligned}
$$

Since markets clear, this allocation can be sustained in equilibrium.

The foregoing analysis does not depend on whether households are naïve or sophisticated. Both types of households would face the same maximization problem. For the analysis of period 1, however, a distinction has to be made between naïves and sophisticates. To avoid confusion, a superscript " $n$ " is added for naïve households and a superscript "s" for sophisticated households.

Suppose that for each period the expected prices of both goods are equal, i.e. $p_{1,1 \mid 1}=p_{1,2 \mid 1}$ and $p_{2,1 \mid 1}=p_{2,2 \mid 1}$. Since the naïve household 1 (2) now cares most about consumption of good 1 (2), the demanded consumption bundles are as follows:

$$
\begin{aligned}
& \left(x_{1,1 \mid 1}^{* n 1}, x_{1,2 \mid 1}^{* n 1}, x_{2,1 \mid 1}^{* n 1}, x_{2,2 \mid 1}^{* n 1}\right)=(2,0,2,0), \\
& \left(x_{1,1 \mid 1}^{* n 2}, x_{1,2 \mid 1}^{* n 2}, x_{2,1 \mid 1}^{* n 2}, x_{2,2 \mid 1}^{* n 2}\right)=(0,2,0,2) .
\end{aligned}
$$

Again, the assumed relative prices can be sustained in equilibrium.

Now the assumption is made that all households are sophisticated. Suppose again that the prices of the two goods are equal in both periods. Demand for sophisticated households is as follows:

$$
\begin{aligned}
& \left(x_{1,1 \mid 1}^{* s 1}, x_{1,2 \mid 1}^{* s 1}, x_{2,1 \mid 1}^{* s 1}, x_{2,2 \mid 1}^{* s 1}\right)=(2,0,0,2), \\
& \left(x_{1,1 \mid 1}^{* s 2}, x_{1,2 \mid 1}^{* s 2}, x_{2,1 \mid 1}^{* s 2}, x_{2,2 \mid 1}^{* s 2}\right)=(0,2,2,0),
\end{aligned}
$$

and again markets clear.

Note that both households are better off if they are both naïve than if they are both sophisticated. This can be seen by calculating their utility levels in both cases.

$$
\begin{aligned}
& \left(u_{1}^{n 1}, u_{2}^{1}\right)=(8,4), \\
& \left(u_{1}^{s 1}, u_{2}^{1}\right)=(6,4), \\
& \left(u_{1}^{n 2}, u_{2}^{2}\right)=(8,4), \\
& \left(u_{1}^{s 2}, u_{2}^{2}\right)=(6,4) .
\end{aligned}
$$


By reallocating the goods in the sophisticated equilibrium, all households can be made strictly better off than at the equilibrium allocations in period 1 . This means that the sophisticated equilibrium is not MOP efficient. Moreover, all households could benefit from being naïve instead of sophisticated. Here the sophisticated households are too forward-looking.

\subsubsection{Constrained Myopic Periodical Efficiency}

In this subsection we assume that a social planner can only reallocate commodities in the current period. This leads to the following definition.

\section{Definition 5.22 Constrained Myopic Periodical (CMP) Efficiency}

The feasible allocation $x^{*}$ is constrained myopic periodically (CMP) efficient if there is no allocation $\tilde{x}$ and no period $t^{\prime}$ such that

(i) $\tilde{x}_{t^{\prime}+1, T \mid t^{\prime}}^{h}=x_{t^{\prime}+1, T \mid t^{\prime}}^{* h}$ for every $h \in H$,

(ii) $\sum_{h \in H} \tilde{x}_{t^{\prime} \mid t^{\prime}}^{h}=\sum_{h \in H} e_{t^{\prime} \mid t^{\prime}}^{h}$,

(iii) $\tilde{x}_{\cdot \mid t^{\prime}}^{h} \succeq_{x_{-\mid t^{\prime}}^{* h}} x_{\cdot \mid t^{\prime}}^{* h}$ for all $h \in H$, and

(iv) $\tilde{x}_{\cdot \mid t^{\prime}}^{h^{\prime}} \succ_{x_{-\mid t^{\prime}}^{* h^{\prime}}} x_{\cdot \mid t^{\prime}}^{* h^{\prime}}$ for some $h^{\prime} \in H$.

The following theorem says that CMP efficiency is weaker than MOP efficiency. Its proof is obvious and is therefore omitted.

Theorem 5.23 If an allocation is MOP efficient, then it is CMP efficient.

A naïve equilibrium allocation is CMP efficient under the assumptions of the foregoing sections.

Theorem 5.24 In an economy $\mathcal{E}$ that satisfies Assumptions 2, 3, and 4, a naïve equilibrium allocation is CMP efficient.

As the next example shows, a sophisticated equilibrium allocation is not necessarily CMP efficient under Assumptions 2, 3, and 4.

\section{Example 5.25}

Consider an economy with two sophisticated households, two commodities and 
two periods. Let the preferences of household 1 be given by

$$
\begin{aligned}
& u_{1}^{1}\left(x_{1 \mid 1}^{1}, x_{2 \mid 1}^{1}\right)=x_{1,1 \mid 1}^{1}+\frac{1}{4} x_{1,2 \mid 1}^{1}+x_{2,1 \mid 1}^{1}+\frac{1}{4} x_{2,2 \mid 1}^{1} \\
& u_{2}^{1}\left(x_{1 \mid 1}^{1}, x_{2 \mid 2}^{1}\right)=\left\{\begin{array}{lll}
x_{2,1 \mid 2}^{1}+\frac{1}{4} x_{2,2 \mid 2}^{1} & \text { if } & x_{1,1 \mid 1}^{1} \leq 1 \\
\frac{1}{4} x_{2,1 \mid 2}^{1}+x_{2,2 \mid 2}^{1} & \text { if } & x_{1,1 \mid 1}^{1}>1
\end{array}\right.
\end{aligned}
$$

Let the preferences of household 2 be given by

$$
\begin{aligned}
& u_{1}^{2}\left(x_{1 \mid 1}^{2}, x_{2 \mid 1}^{2}\right)=\frac{1}{4} x_{1,1 \mid 1}^{2}+x_{1,2 \mid 1}^{2}+\frac{1}{4} x_{2,1 \mid 1}^{2}+x_{2,2 \mid 1}^{2} \\
& u_{2}^{2}\left(x_{1 \mid 1}^{2}, x_{2 \mid 2}^{2}\right)=\left\{\begin{array}{lll}
\frac{1}{4} x_{2,1 \mid 2}^{2}+x_{2,2 \mid 2}^{2} & \text { if } \quad x_{1,2 \mid 1}^{2} \leq 1 \\
x_{2,1 \mid 2}^{2}+\frac{1}{4} x_{2,2 \mid 2}^{2} & \text { if } & x_{1,2 \mid 1}^{2}>1 .
\end{array}\right.
\end{aligned}
$$

Let the endowments be given by $e_{t, l \mid 1}^{h}=1$ for every good $l$, for every period $t$, and for every household $h$.

Consider prices and allocation $\left(p^{*}, x^{*}\right)$, where $p_{\tau, l \mid t}^{*}=1$ for every $l \in L$ and every $t, \tau \in T$ with $t \leq \tau, x_{\cdot \mid 1}^{* 1}=(1,1,2,0), x_{\cdot \mid 1}^{* 2}=(1,1,0,2), x_{\cdot \mid 2}^{* 1}=(2,0)$, and $x_{\cdot \mid 2}^{* 2}=(0,2)$. It can easily be seen that the pair $\left(p^{*}, x^{*}\right)$ constitutes a sophisticated equilibrium.

However, consider the allocation $\tilde{x}$ where $\tilde{x}_{\cdot \mid 1}^{1}=(2,0,2,0), \tilde{x}_{\cdot \mid 1}^{2}=(0,2,0,2)$, $\tilde{x}_{\cdot \mid 2}^{1}=(2,0)$, and $\tilde{x}_{\cdot \mid 2}^{2}=(0,2)$. In the first period both households are better off. Therefore, the sophisticated equilibrium allocation $x^{*}$ is not CMP efficient.

By restricting the degree of time-inconsistency in such a way that consumption decisions do not depend on past consumption, a sophisticated equilibrium allocation is CMP efficient. This is shown in the following theorem.

Theorem 5.26 In an economy $\mathcal{E}$ that satisfies Assumptions 2, 3, 4, and 6, a sophisticated equilibrium allocation is CMP efficient.

Krusell, Kuruşçu, and Smith (2002) consider similar concepts as the ones used in this section, for economies with a representative consumer.

\subsection{Efficiency - the Forward-looking Case}

In this section we consider social planners that care about all selves. A social planner will reallocate consumption only if by doing so he can make one self of 
one household better off, while not making any self of any household worse off. On the one hand we might expect social planners to have more opportunities to improve welfare now, in the sense that there are more selves to be made better off. On the other hand, social planners have less opportunities to improve welfare since preferences depend on past consumption. In the preceding section, a social planner was myopic and therefore allowed to make future selves worse off, but here this is no longer the case. Thus, there is no direct relationship between the concepts in this section and the concepts in the preceding section.

The modeling of forward-looking social planners requires an extension of the preferences of households. A reallocation of commodities in period $t$ affects households in periods $t+1$ and further. Such a reallocation will only be made if no current or future self is made worse off. If we want to check whether future selves will be worse off after the reallocation, we need households to be able to compare consumption bundles with different realized past consumption. In this subsection we will extend the preferences of a household $h$ in period $t$ to preferences with domain $X_{\cdot \mid 1}^{h}$. We denote these preferences by $\succeq^{* h, t}$ and impose the following restriction on them

$$
\begin{aligned}
\left(x_{-\mid t}^{h}, x_{\cdot \mid t}^{h}\right) & \succeq^{* h, t} \quad\left(x_{-\mid t}^{h}, \bar{x}_{\cdot \mid t}^{h}\right) \\
& \text { iff } \\
x_{\cdot \mid t}^{h} & \succeq_{x_{-\mid t}^{h}} \quad \bar{x}_{\cdot \mid t}^{h}
\end{aligned}
$$

for $x_{\cdot \mid t}^{h}, \bar{x}_{\cdot \mid t}^{h} \in X_{\cdot \mid t}^{h}$ and $x_{-\mid t}^{h} \in X_{-\mid t}^{h}$. Notice that on top of comparing consumption bundles with identical past consumption, $\succeq^{* h, t}$ can also be used to compare consumption bundles with different past consumption. The preferences relation $\succeq^{*}$ allows us to tell whether a household prefers a situation where it consumed 4 apples yesterday and it consumes 4 apples today to a situation where it consumed 4 pears yesterday and it consumes 4 pears today, which is impossible with the preference relation $\succeq$. For all properties of preference relations $\succeq_{x_{-\mid t}^{h}}$ that we defined in the first part of this chapter, we will say that $\succeq^{* h, t}$ satisfies these properties if the induced $\succeq_{x_{-\mid t}^{h}}$ satisfy them.

Some of our results require the following version of independence of preferences of past consumption.

\section{Definition 5.27 Strong independence of past consumption}

Preferences $\succeq^{* h, t}$ are strongly independent of past consumption if the following holds: $\left(\widehat{x}_{-\mid t}^{h}, \widehat{x}_{\cdot \mid t}^{h}\right) \succeq^{* h, t}\left(\bar{x}_{-\mid t}^{h}, \bar{x}_{\cdot \mid t}^{h}\right)$ if and only if $\left(x_{-\mid t}^{h}, \widehat{x}_{\cdot \mid t}^{h}\right) \succeq^{* h, t}\left(\breve{x}_{-\mid t}^{h}, \bar{x}_{\cdot \mid t}^{h}\right)$ for every $x_{-\mid t}^{h}, \breve{x}_{-\mid t}^{h} \in X_{-\mid t}^{h}$. 
We replace Assumption 6 by Assumption 6'.

Ass. 6' Preferences $\succeq^{* h, t}$ are strongly independent of past consumption.

This assumption is stronger than Assumption 6. Consider a household with preferences $\succeq^{* h, t}$ represented by $U\left(x_{-\mid t}^{h}, x_{\cdot \mid t}^{h}\right)=\sum_{t^{\prime}<t} \sum_{l \in L} x_{t^{\prime}, l \mid t^{\prime}}^{h}+\sum_{t^{\prime} \geq t} \sum_{l \in L} x_{t^{\prime}, l \mid t}^{h}$. These preferences do satisfy Assumption 6, but not Assumption 6'. With these specific preferences past consumption does not influence current behavior, but past consumption does influence current utility.

We will again first consider social planners that can alter both actual and planned consumption.

\subsubsection{Overall Pareto Efficiency}

In this subsection we consider social planners that can alter both current and planned consumption. Combined with the assumption that social planners care about all selves, the natural extension of the myopic overall Pareto efficiency concept yields the following definition.

\section{Definition 5.28 Overall Pareto (OP) Efficiency}

The feasible allocation $x^{*}$ is overall Pareto (OP) efficient if there is no allocation $\tilde{x}$ and no period $t^{\prime}$ such that

(i) $\sum_{h \in H} \tilde{x}_{\cdot \mid t}^{h}=\sum_{h \in H} e_{\cdot \mid t}^{h}$ for all $t \geq t^{\prime}$,

(ii) $\left(x_{-\mid t^{\prime}}^{* h}, \tilde{x}_{t^{\prime} \mid t^{\prime}}^{h}, \ldots, \tilde{x}_{t-1 \mid t-1}^{h}, \tilde{x}_{\cdot \mid t}^{h}\right) \succeq^{* h, t}\left(x_{-\mid t}^{* h}, x_{\cdot \mid t}^{* h}\right)$ for all $h \in H$ and all $t \geq t^{\prime}$, and

(iii) $\left(x_{-\mid t^{\prime}}^{* h^{\prime}}, \tilde{x}_{t^{\prime} \mid t^{\prime}}^{h^{\prime}}, \ldots, \tilde{x}_{t^{\prime \prime}-1 \mid t^{\prime \prime}-1}^{h^{\prime}}, \tilde{x}_{\cdot \mid t^{\prime \prime}}^{h^{\prime}}\right) \succ^{* h^{\prime}, t^{\prime \prime}}\left(x_{-\mid t^{\prime \prime}}^{* h^{\prime}}, x_{\cdot \mid t^{\prime \prime}}^{* h^{\prime}}\right)$ for some $h^{\prime} \in H$ and some $t^{\prime \prime} \geq t^{\prime}$.

A social planner will reallocate commodities only if by doing so one self of one household is made better off and no self of any household is made worse off. There is no need for a social planner to restrict himself to time-consistent allocations. This concept is therefore not appropriate for the analysis of sophisticated societies. If preferences do not depend on past consumption, OP efficiency corresponds to "weak Pareto efficiency" as discussed in Luttmer and Mariotti (2006). OP efficiency is indeed a weakening of Pareto efficiency.

Theorem 5.29 Assume that preferences are time-consistent and satisfy Assumption 6'. If a time-consistent allocation is Pareto efficient, then it is OP efficient. 
Examples that show that naïve and sophisticated equilibrium allocations may not be OP efficient can easily be constructed.

In sophisticated societies, it makes sense to require a social planner to only change allocations in such a way that the reallocation is time-consistent. This is formalized in the following definition.

\section{Definition 5.30 TC Overall Pareto (TCOP) Efficiency}

The feasible time-consistent allocation $x^{*}$ is time-consistent overall Pareto (TCOP) efficient if there is no time-consistent allocation $\tilde{x}$ and no period $t^{\prime}$ such that

(i) $\sum_{h \in H} \tilde{x}_{\cdot \mid t}^{h}=\sum_{h \in H} e_{\cdot \mid t}^{h}$ for all $t \geq t^{\prime}$,

(ii) $\left(x_{-\mid t^{\prime}}^{* h}, \tilde{x}_{t^{\prime} \mid t^{\prime}}^{h}, \ldots, \tilde{x}_{t-1 \mid t-1}^{h}, \tilde{x}_{\cdot \mid t}^{h}\right) \succeq^{* h, t}\left(x_{-\mid t}^{* h}, x_{. \mid t}^{* h}\right)$ for all $h \in H$ and all $t \geq t^{\prime}$, and

(iii) $\left(x_{-\mid t^{\prime}}^{* h^{\prime}}, \tilde{x}_{t^{\prime} \mid t^{\prime}}^{h^{\prime}}, \ldots, \tilde{x}_{t^{\prime \prime}-1 \mid t^{\prime \prime}-1}^{h^{\prime}}, \tilde{x}_{\cdot \mid t^{\prime \prime}}^{h}\right) \succ^{* h^{\prime}, t^{\prime \prime}}\left(x_{-\mid t^{\prime \prime}}^{* h^{\prime}}, x_{\cdot \mid t^{\prime \prime}}^{* h^{\prime}}\right)$ for some $h^{\prime} \in H$ and some $t^{\prime \prime} \geq t^{\prime}$.

In the case of myopic social planners we did not need to introduce a time-consistent version of MOP efficiency, since those social planners do not care about future selves. The following theorem provides a characterization TCOP efficiency, which is useful for later results. Its proof is obvious and therefore not included in the Appendix.

Theorem 5.31 A feasible time-consistent allocation $x^{*}$ is TCOP efficient if and only if there is no time-consistent allocation $\tilde{x}$ and no period $t^{\prime}$ such that

(i) $\sum_{h \in H} \tilde{x}_{\cdot \mid 1}^{h}=\sum_{h \in H} e_{\cdot \mid 1}^{h}$,

(ii) $\left(x_{-\mid t^{\prime}}^{* h}, \tilde{x}_{\cdot \mid t^{\prime}}^{h}\right) \succeq^{* h, t} x_{\cdot \mid 1}^{* h}$ for all $h \in H$ and all $t \geq t^{\prime}$, and

(iii) $\left(x_{-\mid t^{\prime}}^{* h^{\prime}}, \tilde{x}_{\cdot \mid t^{\prime}}^{h^{\prime}}\right) \succ^{* h^{\prime}, t^{\prime \prime}} x_{\cdot \mid 1}^{* h^{\prime}}$ for some $h^{\prime} \in H$ and some $t^{\prime \prime} \geq t^{\prime}$.

The following results shows that TCOP efficiency is a weakening of Pareto efficiency.

Theorem 5.32 Assume that preferences are time-consistent and satisfy Assumption 6'. If a time-consistent allocation is Pareto efficient then it is TCOP efficient.

Note that a TCOP efficient allocation might not be Pareto efficient, even if preferences are time-consistent and satisfy Assumption 6'. Consider for instance an economy with two households, where one household has a much lower discount 
factor than the other. Consider an initial allocation where consumers have strictly positive endowments both in period 1 and in period 2 . Then a social planner that cares only about the selves in the first period would let one household consume only in the first period and the other only in the second period. If the social planner would also have to take care of future selves of the households, this would not be possible. The household that would not consume in the second period would be better off in the first period, but worse off in the second period.

The following theorem claims that if preferences are independent of past consumption, then a sophisticated equilibrium allocation is TCOP efficient.

Theorem 5.33 In an economy $\mathcal{E}$ that satisfies Assumptions 2, 3, 4, and 6', a sophisticated equilibrium allocation is TCOP efficient.

\subsubsection{Constrained Periodical Efficiency}

In this section we assume that social planners care about all selves, but that a social planner active in period $t$ can only modify consumption in period $t$. This yields the following definition.

\section{Definition 5.34 Constrained Periodical (CP) Efficiency}

The feasible allocation $x^{*}$ is constrained periodically $(C P)$ efficient if there is no other allocation $\tilde{x}$ and no period $t^{\prime}$ such that

(i) $\tilde{x}_{t^{\prime}+1, T \mid t^{\prime}}^{h}=x_{t^{\prime}+1, T \mid t^{\prime}}^{* h}$ for every $h \in H$,

(ii) $\sum_{h \in H} \tilde{x}_{t^{\prime} \mid t^{\prime}}^{h}=\sum_{h \in H} e_{t^{\prime} \mid t^{\prime}}^{h}$,

(iii) $\left(x_{-\mid t^{\prime}}^{* h}, \tilde{x}_{t^{\prime} \mid t^{\prime}}^{h}, x_{t^{\prime}+1 \mid t^{\prime}+1}^{* h}, \ldots, x_{t-1 \mid t-1}^{* h}, x_{\cdot \mid t}^{* h}\right) \succeq^{* h, t}\left(x_{-\mid t}^{* h}, x_{\cdot \mid t}^{* h}\right)$ for all $h \in H$ and all $t \geq t^{\prime}$, and

(iv) $\left(x_{-\mid t^{\prime}}^{* h^{\prime}}, \tilde{x}_{t^{\prime} \mid t^{\prime}}^{h^{\prime}}, x_{t^{\prime}+1 \mid t^{\prime}+1}^{* h^{\prime}}, \ldots, x_{t^{\prime \prime}-1 \mid t^{\prime \prime}-1}^{* h}, x_{\cdot \mid t^{\prime \prime}}^{* h}\right) \succ^{* h^{\prime}, t^{\prime \prime}}\left(x_{-\mid t^{\prime \prime}}^{* h^{\prime}}, x_{\cdot \mid t^{\prime \prime}}^{* h^{\prime}}\right)$ for some $h^{\prime} \in$ $H$ and some $t^{\prime \prime} \geq t^{\prime}$.

As only current consumption can be changed, it is not necessary to introduce a time-consistent variant of this definition as we did in the foregoing section. Contrary to the concept of TCOP efficiency, the CP efficiency concept is also applicable to naïve societies.

The following theorem says that the CP efficiency concept is weaker than the OP efficiency concept. Since its proof is obvious, it is omitted. 
Theorem 5.35 If an allocation is OP efficient, then it is CP efficient.

A similar theorem holds for time-consistent allocations and TCOP efficiency.

Theorem 5.36 If a time-consistent allocation is TCOP efficient, then it is CP efficient.

The next example shows that under Assumptions 2, 3, 4 and 6, naïve and sophisticated equilibria need not be constrained periodically efficient.

\section{Example 5.37}

Consider an economy with two periods, two households and two goods. Let the endowments of all households be equal to 1 for each good in each period, i.e. $e_{. \mid \tau}^{h}=(1,1)$ for all $h, \tau$. For both households the preferences in the first period can be represented by the following utility function:

$$
u_{1}^{h}\left(x_{\cdot \mid 1}^{h}\right)=x_{1,1 \mid 1}^{h}+x_{1,2 \mid 1}^{h}+x_{2,1 \mid 1}^{h}+x_{2,2 \mid 1}^{h} .
$$

In the second period, the preferences of the household are represented by the following utility functions:

$$
\begin{aligned}
& u_{2}^{1}\left(x_{1 \mid 1}^{1}, x_{\cdot \mid 2}^{1}\right)=2 x_{1,1 \mid 1}^{1}+x_{1,2 \mid 1}^{1}+x_{2,1 \mid 2}^{1}+x_{2,2 \mid 2}^{1}, \\
& u_{2}^{2}\left(x_{1 \mid 1}^{2}, x_{\cdot \mid 2}^{2}\right)=x_{1,1 \mid 1}^{2}+2 x_{1,2 \mid 1}^{2}+x_{2,1 \mid 2}^{2}+x_{2,2 \mid 2}^{2} .
\end{aligned}
$$

Consider a price system where the prices of all goods are equal in both periods, i.e. $p_{1,1 \mid 1}^{*}=p_{1,2 \mid 1}^{*}=p_{2,1 \mid 1}^{*}=p_{2,2 \mid 1}^{*}$ and $p_{2,1 \mid 2}^{*}=p_{2,2 \mid 2}^{*}$. The following allocations are consistent with optimizing behavior of both naïve and sophisticated households at the price system $p^{*}$ :

$$
\begin{aligned}
\left(x_{1,1 \mid 1}^{* h}, x_{1,2 \mid 1}^{* h}, x_{2,1 \mid 1}^{* h}, x_{2,2 \mid 1}^{* h}\right) & =(1,1,1,1), \\
\left(x_{2,1 \mid 2}^{* h}, x_{2,2 \mid 2}^{* h}\right) & =(1,1),
\end{aligned}
$$

for every household $h$. Thus, at the price system $p^{*}$, markets clear, and $\left(p^{*}, x^{*}\right)$ is both a naïve and a sophisticated equilibrium. Note, however, that this equilibrium is not $\mathrm{CP}$ efficient. In this equilibrium the first-period utility equals 4 for both households, while the utilities in the second period equal 5. Now consider the allocation

$$
\begin{aligned}
& \left(\tilde{x}_{1,1 \mid 1}^{1}, \tilde{x}_{1,2 \mid 1}^{1}, \tilde{x}_{2,1 \mid 1}^{1}, \tilde{x}_{2,2 \mid 1}^{1}\right)=(2,0,1,1), \\
& \left(\tilde{x}_{1,1 \mid 1}^{2}, \tilde{x}_{1,2 \mid 1}^{2}, \tilde{x}_{2,1 \mid 1}^{2}, \tilde{x}_{2,2 \mid 1}^{2}\right)=(0,2,1,1),
\end{aligned}
$$


where the utility levels in the first period remain equal to 4 for both households, whereas the utilities in the second period both equal 6 under correct expectations. Thus, at the allocation $\tilde{x}$, households are not worse off in the first period, and better off in the second period. Moreover, the allocation $\tilde{x}$ is feasible. Therefore, the equilibrium allocation $x^{*}$ is not CP efficient.

If preferences are strongly independent of the past, then naïve and sophisticated equilibria are constrained periodically efficient.

Theorem 5.38 In an economy $\mathcal{E}$ that satisfies Assumptions 2, 3, 4, and 6', naïve and sophisticated equilibrium allocations are CP efficient.

The results of the last two sections are summarized in Figures 5.1 and 5.2. An arrow from "A" to "B" means that A implies B. Labels attached to arrows specify under which assumptions the implications hold true.

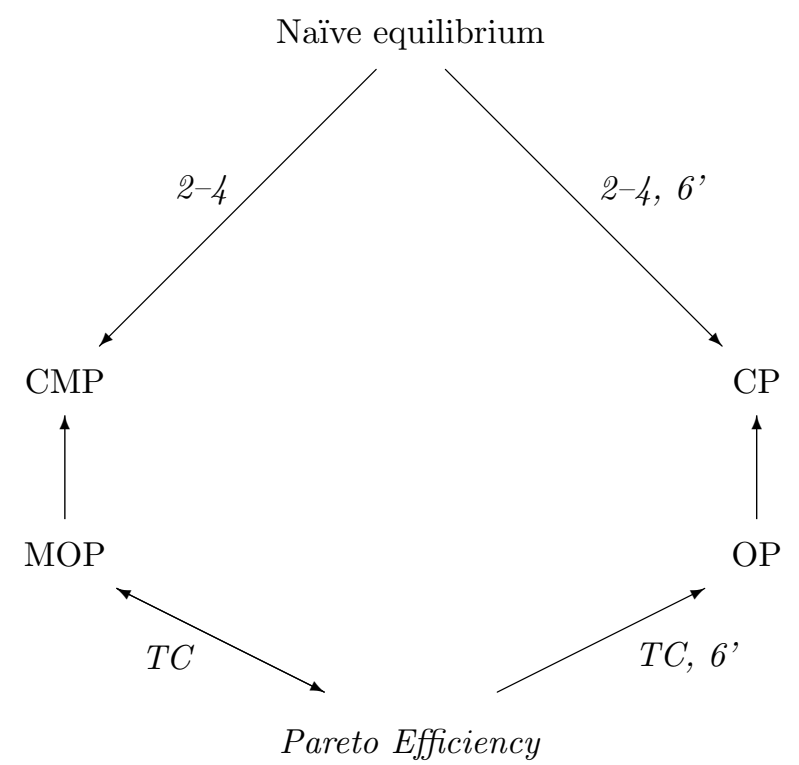

Figure 5.1: Naïve societies 


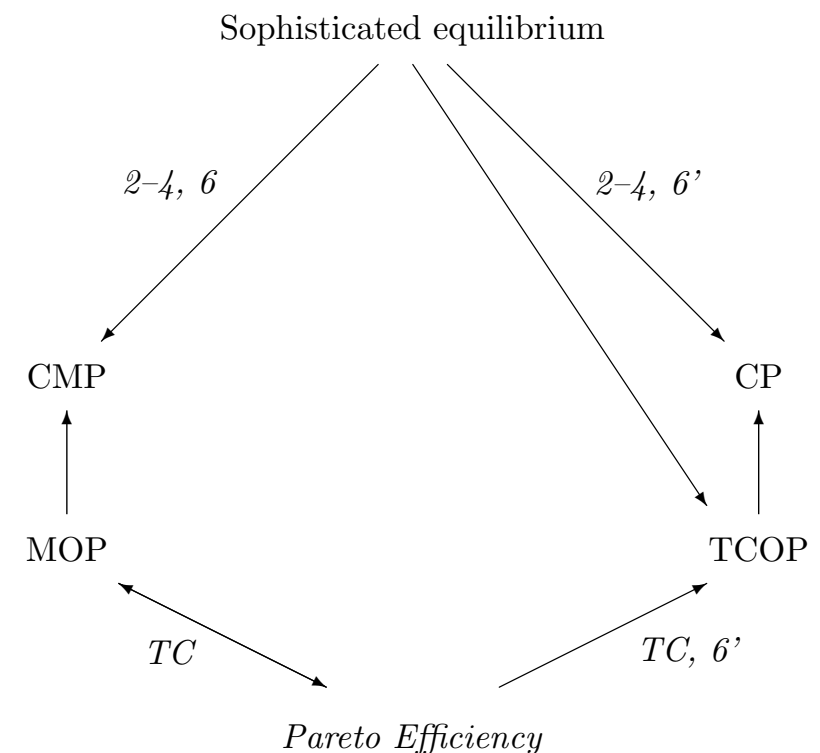

Figure 5.2: Sophisticated societies

\subsection{Conclusion}

In this chapter, changing preferences are introduced in a multi-period general equilibrium model with incomplete markets. Time-inconsistent preferences lead to the development of new concepts of household behavior, equilibrium, and efficiency. A distinction is made between naïve and sophisticated societies. Appropriate equilibrium notions are defined. We extend the standard competitive equilibrium notion and call it an extended competitive equilibrium. It is shown that, in the case of time-consistent preferences, an extended competitive equilibrium is a naïve equilibrium and a sophisticated equilibrium coincides with an extended competitive equilibrium. An intriguing result is that with time-consistent preferences there can be naïve equilibrium allocations that are not compatible with any competitive equilibrium. For naïve societies an equilibrium is shown to exist under quite general conditions. For sophisticated societies the existence of an equilibrium can only be established when certain assumptions on the degree of time-inconsistency are made. 
Several efficiency criteria are introduced. A distinction is made between efficiency concepts that take into account only the preferences of the current selves and concepts that take into account the preferences of both the current and future selves. Moreover, we distinguish the cases where only current consumption can be altered to make Pareto improvements and cases where both current and planned consumption can be changed. Suppose only current consumption can be altered. Then we provide sufficient conditions for both naïve and sophisticated equilibria to be efficient. Suppose both current and planned consumption can be changed. Then naïve equilibria are typically not efficient. If future selves are not taken into account, then sophisticated equilibria are not efficient either. For sophisticated equilibria we provide sufficient conditions for efficiency when future selves are taken into account.

\subsection{Appendix}

ProOF OF LeMMA 5.3

Let $x_{-\mid t}^{h} \in X_{-\mid t}^{h}$. We first show that when $x_{\cdot \mid t}^{h}, \bar{x}_{\cdot \mid t}^{h} \in X_{\cdot \mid t}^{h}$ are such that $x_{t, t^{\prime}-1 \mid t}^{h}=$ $\bar{x}_{t, t^{\prime}-1 \mid t}^{h}$, then

$$
x_{\cdot \mid t}^{h} \quad \succeq_{x_{-\mid t}^{h}}^{h, t} \quad \bar{x}_{\cdot \mid t}^{h}
$$

implies

$$
x_{t^{\prime}, T \mid t}^{h} \quad \succeq_{\left(x_{-\mid t}^{h}, x_{t, t^{\prime}-1 \mid t}^{h}\right)}^{h, t^{\prime}} \quad \bar{x}_{t^{\prime}, T \mid t}^{h}
$$

If $x_{\cdot \mid t}^{h} \succeq_{x_{-\mid t}^{h}}^{h, t} \bar{x}_{\cdot \mid t}^{h}$, then it follows from the "if" part of the hypothesis of the lemma that

$$
\left(x_{-\mid t}^{h}, x_{\cdot \mid t}^{h}\right) \succeq^{h, 1}\left(x_{-\mid t}^{h}, \bar{x}_{\cdot \mid t}^{h}\right) .
$$

The "only if" part of the hypothesis yields

$$
x_{t^{\prime}, T \mid t}^{h} \succeq_{\left(x_{-\mid t}^{h}, x_{t, t^{\prime}-1 \mid t}^{h}\right)}^{h, t^{\prime}} \bar{x}_{t^{\prime}, T \mid t}^{h} .
$$

The proof that

$$
x_{t^{\prime}, T \mid t}^{h} \succeq_{\left(x_{-\mid t}^{h}, x_{t, t^{\prime}-1 \mid t}^{h}\right)}^{h, t^{\prime}} \bar{x}_{t^{\prime}, T \mid t}^{h}
$$

implies

$$
x_{\cdot \mid t}^{h} \succeq_{x_{-\mid t}^{h}}^{h, t} \bar{x}_{\cdot \mid t}^{h}
$$


is similar.

\section{Proof of Theorem 5.7}

Suppose $\left(p^{*}, x^{*}\right)$ is an extended competitive equilibrium. Since $x_{\tau \mid t}^{* h}=x_{\tau \mid 1}^{* h}$ for every $h$ and every $\tau \geq t$ and $\sum_{h \in H} x_{\cdot \mid 1}^{* h}=\sum_{h \in H} e_{\cdot \mid 1}^{h}$, it can easily be seen that (b) of Definition 5.6 is satisfied. It remains to be shown that Condition (a) of that definition is satisfied.

Notice that if $x_{\cdot \mid 1}^{* h} \in \gamma_{1}^{h}\left(p_{\cdot \mid 1}^{*}\right)$, then $x_{t, T \mid 1}^{* h} \in \gamma_{t}^{h}\left(p_{t, T \mid 1}^{*}\right)$. Moreover, for every $x_{\cdot \mid t}^{h} \in \gamma_{t}^{h}\left(p_{t, T \mid 1}^{*}\right)$ there is $x_{\cdot \mid 1}^{h} \in \gamma_{1}^{h}\left(p_{\cdot \mid 1}^{*}\right)$ with $x_{t, T \mid 1}^{h}=x_{\cdot \mid t}^{h}$ and $x_{1, t-1 \mid 1}^{h}=x_{1, t-1 \mid 1}^{* h}$.

We know that $x_{\cdot \mid 1}^{* h} \in \delta_{1}^{h}\left(p_{\cdot \mid 1}^{*}\right)$. Thus, $x_{\cdot \mid 1}^{* h} \in \gamma_{1}^{h}\left(p_{\cdot \mid 1}^{*}\right)$ and $x_{\cdot \mid 1}^{* h} \succeq^{h, 1} x_{\cdot \mid 1}^{h}$ for all $x_{\cdot \mid 1}^{h} \in \gamma_{1}^{h}\left(p_{\cdot \mid 1}^{*}\right)$. Then, by time-consistency of preferences, $x_{t, T \mid 1}^{* h} \succeq_{x_{1, t-1 \mid 1}^{* h}}^{h, t} x_{\cdot \mid t}^{h}$ for all $x_{\cdot \mid t}^{h} \in \gamma_{t}^{h}\left(p_{t, T \mid 1}^{*}\right)$, so $x_{t, T \mid 1}^{* h} \in \delta_{t}^{h}\left(p_{t, T \mid 1}^{*}, x_{1, t-1 \mid 1}^{* h}\right)=\delta_{t}^{h}\left(p_{\cdot \mid t}^{*}, x_{-\mid t}^{* h}\right)$. Thus, the extended competitive equilibrium is a naïve equilibrium.

\section{Proof of Theorem 5.9}

This follows immediately from the definitions.

\section{Proof of Theorem 5.10}

We follow the approach of Debreu (1959) compounded with an induction argument.

First, let $\varepsilon>0$ and define

$$
\widehat{X}_{\tau \mid t}^{h}=\left\{x_{\tau \mid t}^{h} \in X_{\tau \mid t}^{h} \mid x_{\tau, \mid l t}^{h} \leq \sum_{h \in H} e_{\tau, l \mid t}^{h}+\varepsilon \text { for all } l \in L\right\}
$$

for some $\varepsilon>0$. Let $\widehat{\gamma}_{t}^{h}$ and $\widehat{\delta}_{t}^{h}$ denote the corresponding budget and demand correspondences. The economy $\widehat{\mathcal{E}}$ is the compactified economy. We derive some properties of the demand correspondence $\widehat{\delta}^{h}$.

We denote the $(L-1)$-dimensional unit simplex by $\Delta$, so $\Delta=\left\{p \in \mathbb{R}_{+}^{L} \mid\right.$ $\left.\sum_{l=1}^{L} p_{l}=1\right\}$, and we denote the $k$-fold Cartesian product of $\Delta$ by $\Delta^{k}$. The price vectors are restricted to the sets $\widehat{P}_{. \mid t}=\Delta^{T-t+1}$.

In the next lemma the box product in $p_{\cdot \mid t} \square \delta_{t}^{h}\left(p_{\cdot \mid t}, x_{-\mid t}^{h}\right)$ is defined by taking for all $\tau \geq t$ the product of $p_{\tau \mid t}$ and any demand $x_{\tau \mid t}^{h}$ planned at period $t$ for period $\tau$, i.e.

$$
\begin{aligned}
p_{\cdot \mid t} \square \delta_{t}^{h}\left(p_{\cdot \mid t}, x_{-\mid t}^{h}\right)=\left\{\left(w_{t}, \ldots, w_{T}\right) \in \mathbb{R}^{T-t+1} \mid\right. \\
\text { there is an } \left.x_{\cdot \mid t}^{h} \in \delta_{t}^{h}\left(p_{\cdot \mid t}, x_{-\mid t}^{h}\right) \text { such that } w_{\tau}=p_{\tau \mid t} x_{\tau \mid t}^{h}, \tau \in t, \ldots, T\right\} .
\end{aligned}
$$

Lemma 5.39 Assume that the economy $\mathcal{E}$ satisfies Assumptions 1-5. Consider a naïve household $h \in H$, a planning period $t \in T$, and a realized consumption plan 
$x_{-\mid t}^{h} \in \widehat{X}_{-\mid t}^{h}$. Then, at prices $p_{\cdot \mid t} \in \widehat{P}_{\cdot \mid t}, \widehat{\delta}_{t}^{h}\left(\cdot, x_{-\mid t}^{h}\right)$ is a non-empty, compact and convex-valued, upper-hemi continuous correspondence that satisfies:

- Walras' law, $p_{\cdot \mid t} \square \widehat{\delta}_{t}^{h}\left(p_{\cdot \mid t}, x_{-\mid t}^{h}\right)=\left\{p_{t \mid t} e_{t \mid t}^{h}, \ldots, p_{T \mid t} e_{T \mid t}^{h}\right\}$,

- Homogeneity property, $\widehat{\delta}_{t}^{h}\left(p_{\cdot \mid t}, x_{-\mid t}^{h}\right)=\widehat{\delta}_{t}^{h}\left(\bar{p}_{\cdot \mid t}, x_{-\mid t}^{h}\right)$, where for $\tau^{\prime} \geq t$, for $\lambda>0, \bar{p}_{\tau^{\prime} \mid t}=\lambda p_{\tau^{\prime} \mid t}$ and $\bar{p}_{\tau \mid t}=p_{\tau \mid t}$ for $\tau \neq \tau^{\prime}$.

\section{Proof}

(i) Since $e_{\cdot \mid t}^{h} \in \widehat{\gamma}_{t}^{h}\left(p_{\cdot \mid t}\right)$, we know that $\widehat{\gamma}_{t}^{h}\left(p_{\cdot \mid t}\right)$ is non-empty.

(ii) Consider a sequence $\left\{p_{\cdot \mid t}{ }^{m}\right\}_{m=1}^{\infty}$ with $p_{\cdot \mid t}{ }^{m} \rightarrow p_{\cdot \mid t}$. Let the sequence $\left\{x_{\cdot \mid t}^{h}\right\}_{m=1}^{m}$ be such that $x_{\cdot \mid t}^{h} \in \widehat{\gamma}_{t}^{h}\left(p_{\cdot \mid t}{ }^{m}\right)$ for every $m$ and $x_{\cdot \mid t}^{h} \rightarrow x_{\cdot \mid t}^{h}$. By closedness of $\widehat{X}_{\cdot \mid t}^{h}$ and since $p_{\tau \mid t}{ }^{m} x_{\tau \mid t}^{h}{ }^{m} \leq p_{\tau \mid t}{ }^{m} e_{\tau \mid t}^{h}$, it follows that $x_{\cdot \mid t}^{h} \in \widehat{\gamma}_{t}^{h}\left(p_{\cdot \mid t}\right)$. Since $\widehat{\gamma}_{t}^{h}$ is bounded, it follows that $\widehat{\gamma}_{t}^{h}$ is upper-hemi continuous.

(iii) Let $\left\{p_{\cdot \mid t}{ }^{m}\right\}_{m=1}^{\infty}$ be a sequence of prices with $p_{\cdot \mid t}{ }^{m} \rightarrow p_{\cdot \mid t}$. Let $x_{\cdot \mid t}^{h} \in \widehat{\gamma}_{t}^{h}\left(p_{\cdot \mid t}\right)$. Then $p_{\tau \mid t} x_{\tau \mid t}^{h} \leq p_{\tau \mid t} e_{\tau \mid t}^{h}$. Define $a^{\tau m} \in \mathbb{R}_{++}$such that $p_{\tau \mid t}{ }^{m} a^{\tau m} x_{\tau \mid t}^{h}=p_{\tau \mid t}{ }^{m} e_{\tau \mid t}^{h}$.

If $p_{\tau \mid t} x_{\tau \mid t}^{h}<p_{\tau \mid t} e_{\tau \mid t}^{h}$, then $p_{\tau \mid t}{ }^{m} x_{\tau \mid t}^{h} \leq p_{\tau \mid t}{ }^{m} e_{\tau \mid t}^{h}$ for $m$ larger than a certain value $M^{1}$. In that case define $x_{\tau \mid t}^{h}{ }^{m}=x_{\tau \mid t}^{h}$ for $m>M^{1}$.

Otherwise, if $p_{\tau \mid t} x_{\tau \mid t}^{h}=p_{\tau \mid t} e_{\tau \mid t}^{h}>0$, it holds that $p_{\tau \mid t}{ }^{m} e_{\tau \mid t}^{h}>0$ and $p_{\tau \mid t}{ }^{m} x_{\tau \mid t}^{h}>$ 0 for $m$ larger than a certain $M^{2}$. Now, if $a^{\tau m}>1$, then define $x_{\tau \mid t}^{h}{ }^{m}=x_{\tau \mid t}^{h}$ and if $a^{\tau m} \leq 1$, then define $x_{\tau \mid t}^{h}{ }^{m}=a^{\tau m} x_{\tau \mid t}^{h}$ for $m$ larger than $M^{2}$. Note that in this case $a^{\tau m}$ is unique and tends to one, since $a^{\tau m}=p_{\tau \mid t}{ }^{m} e_{\tau \mid t}^{h} / p_{\tau \mid t} x_{\tau \mid t}^{h}>0$.

For all $m$ smaller than or equal to $M^{1}$ or $M^{2}$ define $x_{\cdot \mid t}^{h}$ arbitrarily such that $x_{\cdot \mid t}^{h} \in \widehat{\gamma}_{t}^{h}\left(p_{\cdot \mid t}^{m}\right)$.

Then $x_{\cdot \mid t}^{h} \in \widehat{\gamma}_{t}^{h}\left(p_{\cdot \mid t}{ }^{m}\right)$ for every $m$ and $x_{\cdot \mid t}^{h}{ }^{m} \rightarrow x_{\cdot \mid t}^{h}$. Thus, $\widehat{\gamma}_{t}^{h}$ is lower-hemi continuous. It follows that $\widehat{\gamma}_{t}^{h}$ is continuous.

We can then apply the Theorem of the Maximum to establish that $\widehat{\delta}_{t}^{h}\left(\cdot, x_{-\mid t}^{h}\right)$ is non-empty, compact-valued and upper-hemi continuous.

Convex-valuedness of $\widehat{\delta}_{t}^{h}$ is straightforward. Walras' law follows from monotonicity. The homogeneity property follows immediately from the definition of the budget constraints $\widehat{\gamma}_{t}^{h}$.

\section{Proof of Theorem 5.10 (CONTINUed)}

Define $\widehat{Z}_{\cdot \mid t}=\sum_{h \in H} \widehat{X}_{\cdot \mid t}^{h}-\sum_{h \in H}\left\{e_{\cdot \mid t}^{h}\right\}$ and for any $x_{-\mid t} \in \widehat{X}_{-\mid t}$,

$\widehat{\zeta}_{t}\left(p_{\cdot \mid t}, x_{-\mid t}\right)=\sum_{h \in H} \widehat{\delta}_{t}^{h}\left(p_{\cdot \mid t}, x_{-\mid t}^{h}\right)-\sum_{h \in H}\left\{e_{\cdot \mid t}^{h}\right\}$. Using Lemma 5.39, the correspondence $\widehat{\zeta}_{t}\left(\cdot, x_{-\mid t}\right)$ is non-empty, compact-valued, convex-valued and upper-hemi continuous on $\widehat{P}_{\cdot \mid t}$. 
Define $\mu_{t}\left(z_{. \mid t}\right)=\left\{\tilde{p}_{\cdot \mid t} \in \widehat{P}_{. \mid t} \mid \tilde{p}_{\tau \mid t} z_{\tau \mid t} \geq p_{\tau \mid t} z_{\tau \mid t}\right.$ for all $p_{. \mid t} \in \widehat{P}_{. \mid t}$ for all $\left.\tau \geq t\right\}$. By the theorem of the maximum, $\mu_{t}$ is non-empty and upper-hemi continuous. Moreover, $\mu_{t}$ is convex-valued. For $x_{-\mid t} \in \widehat{X}_{-\mid t}$, define $\phi_{t}\left(\cdot, x_{-\mid t}\right): \widehat{P}_{t} \times \widehat{Z}_{t} \rightarrow$ $\widehat{P}_{t} \times \widehat{Z}_{t}$ as $\phi_{t}\left(p_{\cdot \mid t}, z_{\mid t}, x_{-\mid t}\right)=\mu_{t}\left(z_{\cdot \mid t}\right) \times \widehat{\zeta}_{t}\left(p_{\cdot \mid t}, x_{-\mid t}\right)$.

First, consider period 1. By Kakutani's fixed point theorem $\phi_{1}(\cdot)$ has a fixed point $\left(p_{\cdot \mid 1}^{*}, \bar{z}_{\cdot \mid 1}\right) \in \mu_{1}\left(\bar{z}_{|| 1}\right) \times \widehat{\zeta}_{\cdot \mid 1}\left(p_{\cdot \mid t}^{*}\right)$.

Since then $p_{\tau \mid 1}^{*} \bar{z}_{\tau \mid 1} \leq 0$ for every $\tau$, we know, by the definition of $\mu_{1}$, that $\bar{z}_{\cdot \mid 1} \leq 0$. The corresponding consumption bundles are denoted by $\bar{x}_{\cdot \mid 1}^{h} \in \widehat{\delta}_{1}^{h}\left(p_{\cdot \mid 1}^{*}\right)$.

By Walras' law (Lemma 5.39), we know that $p_{\tau, l \mid 1}^{*}=0$ if $\bar{z}_{\tau, l \mid 1}<0$. By monotonicity, the excess supply of good $l$ for period $\tau$ can be given to any household without making that household worse off and without violating the budget constraints. Thus, given prices $p_{\cdot \mid 1}^{*}, z_{\cdot \mid 1}^{*}=0 \in \widehat{\zeta}_{1}\left(p_{\cdot \mid 1}^{*}\right)$. Denote the corresponding demands by $x_{\cdot \mid 1}^{* h}$.

It remains to be shown that $x_{\cdot \mid 1}^{* h} \in \delta_{1}^{h}\left(p_{\cdot \mid 1}^{*}\right)$ for every $h$. Suppose that this is not the case, i.e. suppose that there is a household $h$ with $x_{\cdot \mid 1}^{* h} \notin \delta_{1}^{h}\left(p_{\cdot \mid 1}^{*}\right)$. That would mean that there is an $\tilde{x}_{\cdot \mid 1}^{h} \in \delta_{1}^{h}\left(p_{\cdot \mid 1}^{*}\right)$ with $\tilde{x}_{\cdot \mid 1}^{h} \succ^{h, 1} x_{. \mid 1}^{* h}$. Since $x_{\tau, l \mid 1}^{* h}<$ $\sum_{h \in H} e_{\tau, l \mid 1}^{h}+\varepsilon$ for every $\tau$, and every $l$, there would be a small positive number $\lambda \in(0,1)$ such that $\lambda \tilde{x}_{\cdot \mid 1}^{h}+(1-\lambda) x_{\cdot \mid 1}^{* h} \in \widehat{\gamma}_{1}^{h}\left(p_{\cdot \mid 1}^{*}\right)$, and $\lambda \tilde{x}_{\cdot \mid 1}^{h}+(1-\lambda) x_{\cdot \mid 1}^{* h} \succ^{h, 1} x_{\cdot \mid 1}^{* h}$, which would contradict $x_{\cdot \mid 1}^{* h} \in \widehat{\delta}_{1}^{h}\left(p_{\cdot \mid 1}^{*}\right)$. Thus, $x_{\cdot \mid 1}^{* h} \in \delta_{1}^{h}\left(p_{\cdot \mid 1}^{*}\right)$ for every $h$.

Now suppose that for every $\tau \leq t$ there exist $p_{\cdot \mid \tau}^{*}$ such that $0 \in \zeta_{\tau}\left(p_{\cdot \mid \tau}^{*}, x_{-\mid \tau}^{*}\right)$. Then, by a similar argument as before it can be shown that there exists a $p_{\cdot \mid t+1}^{*}$ such that $0 \in \zeta_{t+1}\left(p_{|| t+1}^{*}, x_{-\mid t+1}^{*}\right)$. This argument of induction then establishes the existence of a naïve equilibrium.

\section{Proof of Theorem 5.12}

It can immediately be seen that a sophisticated equilibrium pair $\left(p^{*}, x^{*}\right)$ satisfies (i)-(iv). It remains to be shown that a pair that satisfies (i)-(iv) is a sophisticated equilibrium. Let $\left(p^{*}, x^{*}\right)$ satisfy (i)-(iv) and let $t<t^{\prime}$. Then, by (iii) $p_{\cdot \mid t}^{*}=p_{t, T \mid 1}^{*}$ and $p_{\cdot \mid t^{\prime}}^{*}=p_{t^{\prime}, T \mid 1}^{*}$. So $p_{t^{\prime}, T \mid t}^{*}=p_{\cdot \mid t^{\prime}}^{*}$ and (c) is satisfied. Furthermore, $x_{\cdot \mid t}^{*}=x_{t, T \mid 1}^{*}$ and $x_{\cdot \mid t^{\prime}}^{*}=x_{t^{\prime}, T \mid 1}^{*}$. So $x_{t^{\prime}, T \mid t}^{*}=x_{\cdot \mid t^{\prime}}^{*}$ and (d) is satisfied. It also holds that $\sum_{h \in H} x_{\cdot \mid t}^{* h}=\sum_{h \in H} x_{t, T \mid 1}^{* h}=\sum_{h \in H} e_{t, T \mid 1}^{h}=\sum_{h \in H} e_{\cdot \mid t}^{h}$, so (b) is satisfied. Finally, $x_{. \mid 1}^{* h} \in \xi_{1}^{h}\left(p_{\cdot \mid 1}^{*}\right)$, so $x_{\cdot \mid 1}^{* h} \in \phi_{1}^{h}\left(p_{\cdot \mid 1}^{*}\right)$, which implies that $x_{2, T \mid 1}^{* h} \in \xi_{2}^{h}\left(p_{2, T \mid 1}^{*}, x_{1 \mid 1}^{* h}\right)$. But then, $x_{\cdot \mid 2}^{* h} \in \xi_{2}^{h}\left(p_{\cdot \mid 2}^{*}, x_{1 \mid 1}^{* h}\right)$. Now, by an argument of induction it can be shown that $x_{. \mid t}^{* h} \in \xi_{t}^{h}\left(p_{|| t}^{*}, x_{-\mid t}^{* h}\right)$ for all t. So (a) is satisfied too. Thus, a pair $\left(p^{*}, x^{*}\right)$ that satisfies (i)-(iv) is a sophisticated equilibrium. 


\section{Proof OF THEOREM 5.13}

First of all, let $\left(p^{*}, x^{*}\right)$ be an extended competitive equilibrium. Since $x_{\cdot \mid 1}^{* h} \in$ $\delta_{1}^{h}\left(p_{\cdot \mid 1}^{*}\right)$ we know that $x_{\cdot \mid 1}^{* h} \succeq^{h, 1} x_{\cdot \mid 1}^{h}$ for every $x_{\cdot \mid 1}^{h} \in X_{\cdot \mid 1}^{h}$ with $p_{\tau \mid 1}^{*} x_{\tau \mid 1}^{h} \leq p_{\tau \mid 1}^{*} e_{\tau \mid 1}^{h}$ for every $\tau$. By time-consistency we then know that $x_{T \mid 1}^{* h} \succeq_{x_{1, T-1 \mid 1}^{* h}}^{h, T} x_{T \mid 1}^{h}$ for every $x_{T \mid 1}^{h} \in X_{\cdot \mid T}^{h}$ with $p_{T \mid 1}^{*} x_{T \mid 1}^{h} \leq p_{T \mid 1}^{*} e_{T \mid 1}^{h}$. It follows that $x_{T \mid 1}^{* h} \in \xi_{T}^{h}\left(p_{T \mid 1}^{*}, x_{1, T-1 \mid 1}^{* h}\right)$. We show next that $x_{t, T \mid 1}^{* h} \in \xi_{t}^{h}\left(p_{t, T \mid 1}^{*}, x_{1, t-1 \mid 1}^{* h}\right)$ for every $t$. Assume that $x_{\tau, T \mid 1}^{* h} \in$ $\xi_{\tau}^{h}\left(p_{\tau, T \mid 1}^{*}, x_{1, \tau-1 \mid 1}^{* h}\right)$ for every $\tau>t$. Suppose that $x_{t, T \mid 1}^{* h} \notin \xi_{t}^{h}\left(p_{t, T \mid 1}^{*}, x_{1, t-1 \mid 1}^{* h}\right)$. Then there must be a consumption bundle that is strictly preferred to $x_{t, T \mid 1}^{* h}$, but is in the opportunity set at time $t$, which, by time-consistency, leads to a contradiction of $x_{\cdot \mid 1}^{* h}$ being an optimal consumption bundle for household $h$ in period 1. Thus, $\left(p^{*}, x^{*}\right)$ is a sophisticated equilibrium.

Now let $\left(p^{*}, x^{*}\right)$ be a sophisticated equilibrium. Suppose that $\left(p^{*}, x^{*}\right)$ is not an extended competitive equilibrium. Then there must be a household $h$ and an $\widehat{x}_{\cdot \mid 1}^{h} \in X_{\cdot \mid 1}^{h}$ such that $\widehat{x}_{\cdot \mid 1}^{h} \succ^{h, 1} x_{\cdot \mid 1}^{* h}$ with $p_{\tau \mid 1}^{*} \widehat{x}_{\tau \mid 1}^{h} \leq p_{\tau \mid 1}^{*} e_{\tau \mid 1}^{h}$ for every $\tau$. Consider the maximum of those $\widehat{x}_{\cdot \mid 1}^{h}$ with respect to $\succ^{h, 1}$. Such an $\widehat{x}_{\cdot \mid 1}^{h}$ exists because preferences are continuous. Since $\widehat{x}_{\cdot \mid 1}^{h}$ is not chosen by $h$, there must be a $t_{1}>1$ such that $\widehat{x}_{t_{1}, T \mid 1}^{h} \notin \xi_{t_{1}}^{h}\left(p_{t_{1}, T \mid 1}^{*}, \widehat{x}_{1, t_{1}-1 \mid 1}^{h}\right)$. So there must be a $t_{1}^{\prime} \geq t_{1}$ and an $\tilde{x}_{\cdot \mid t_{1}^{\prime}}^{h} \in X_{\cdot \mid t_{1}^{\prime}}^{h}$ such that $\tilde{x}_{\cdot \mid t_{1}^{\prime}}^{h} \succ_{\widehat{x}_{1, t_{1}^{\prime}-1 \mid 1}^{h}}^{h, t_{1}^{\prime}} \widehat{x}_{t_{1}^{\prime}, T \mid 1}^{h}$ and $p_{\tau \mid 1}^{*} \tilde{x}_{\tau \mid t_{1}^{\prime}}^{h} \leq p_{\tau \mid 1}^{*} e_{\tau \mid 1}^{h}$ for every $\tau \geq t_{1}^{\prime}$, and by time-consistency $\left(\widehat{x}_{1, t_{1}^{\prime}-1 \mid 1}^{h}, \tilde{x}_{\cdot \mid t_{1}^{\prime}}^{h}\right) \succ^{h, 1} \widehat{x}_{\cdot \mid 1}^{h} \succ^{h, 1} x_{\cdot \mid 1}^{* h}$, which contradicts our assumption on $\widehat{x}_{\cdot \mid 1}^{h}$.

\section{EXAMPLE 5.15}

An important role is played by the second derivative of $v$ with respect to $x_{1,1 \mid 1}^{1}$. It is given by

$$
\frac{d^{2} v}{d x_{1,1 \mid 1}^{1}{ }^{2}}=\frac{p_{2,1 \mid 2}+p_{2,2 \mid 2}}{\left(1+x_{1,1 \mid 1}^{1}\right)^{3}}\left(\frac{2}{p_{2,1 \mid 2}}-\frac{1}{p_{2,2 \mid 2}}\right)
$$

for $x_{1,1 \mid 1}^{1}>1 / 3$ and is equal to 0 otherwise. Thus, when $x_{1,1 \mid 1}^{1}>1 / 3$, it depends on the prices whether $v$ is convex $\left(p_{2,1 \mid 2}<2 p_{2,2 \mid 2}\right)$, linear $\left(p_{2,1 \mid 2}=2 p_{2,2 \mid 2}\right)$ or concave $\left(p_{2,1 \mid 2}>2 p_{2,2 \mid 2}\right)$. We distinguish three cases accordingly.

Case 1

First consider the case where $p_{2,1 \mid 2}<2 p_{2,2 \mid 2}$. Note first that in this case the second derivative of $v$ with respect to $x_{1,1 \mid 1}^{1}$ is larger than 0 for $x_{1,1 \mid 1}^{1}>1 / 3$ and equal to 0 if $x_{1,1 \mid 1}^{1} \leq 1 / 3$.

First, assume that $2 p_{1,2 \mid 1}<p_{1,1 \mid 1}$, so that the first derivative of $v$ is negative 
when $x_{1,1 \mid 1}^{1}>1 / 3$, i.e.

$$
\frac{d v}{d x_{1,1 \mid 1}^{1}}=1-\frac{p_{1,1 \mid 1}}{2 p_{1,2 \mid 1}}+\frac{p_{2,1 \mid 2}+p_{2,2 \mid 2}}{\left(1+x_{1,1 \mid 1}^{1}\right)^{2}}\left(\frac{1}{2 p_{2,2 \mid 2}}-\frac{1}{p_{2,1 \mid 2}}\right)<0
$$

for $x_{1,1 \mid 1}^{1}>1 / 3$. It is easily verified that the first derivative of $v$ is negative as well for $x_{1,1 \mid 1}^{1} \leq 1 / 3$. Then $x_{1,1 \mid 1}^{* 1}=0$. Note that if $x_{1,1 \mid 1}^{* 1}=0$, then in equilibrium it must hold that $x_{1,1 \mid 1}^{* 2}=1$, which is only possible if $p_{1,1 \mid 1}=0$. However, then demand for goods would be infinite in the first period, which cannot yield an equilibrium.

Now assume that $2 p_{1,2 \mid 1} \geq p_{1,1 \mid 1}$. Since the second derivative of $v$ is larger than 0 if $x_{1,1 \mid 1}^{1}>1 / 3$, it holds that $v$ is convex for $x_{1,1 \mid 1}^{1}>1 / 3$. Thus, the optimal $x_{1,1 \mid 1}^{1}$ will not be an interior solution if $x_{1,1 \mid 1}^{1}>1 / 3$. Thus, either $x_{1,1 \mid 1}^{* 1} \in[0,1 / 3]$ or $x_{1,1 \mid 1}^{* 1}=1$. If $x_{1,1 \mid 1}^{* 1}=1$ then $p_{1,2 \mid 1}=0$, since otherwise household 2 would never be willing to consume $x_{1,1 \mid 1}^{* 2}=0$. But if $p_{1,2 \mid 1}=0$, then household 1 will demand an infinite amount of good 2 in period 1 . Thus, this can also not yield an equilibrium. So consider $x_{1,1 \mid 1}^{* 1} \in[0,1 / 3]$. Then, for the second period demand it holds that $x_{2,1 \mid 2}^{* 1}>9 / 4$. However, it also holds that $x_{2,1 \mid 2}^{* 2}=1$. Thus, there would be excess demand of good 1 in period 2 .

Case 2

Now consider the case where $p_{2,1 \mid 2}>2 p_{2,2 \mid 2}$. Then $x_{2,2 \mid 2}^{* 2}=\frac{p_{2,1 \mid 2}}{p_{2,2 \mid 2}}$ and $x_{2,1 \mid 2}^{* 2}=0$. So in equilibrium it would have to hold that $x_{2,1 \mid 2}^{* 1}=2$ and $x_{2,2 \mid 2}^{* 1}=\frac{p_{2,2 \mid 2}-p_{2,1 \mid 2}}{p_{2,2 \mid 2}}$. First of all, assume that $x_{1,1 \mid 1}^{* 1}>1 / 3$. This would imply the following two conditions:

$$
\begin{aligned}
& \left(1+\frac{p_{2,2 \mid 2}}{p_{2,1 \mid 2}}\right) \frac{1}{1+x_{1,1 \mid 1}^{* 1}}=2, \\
& \left(\frac{p_{2,1 \mid 2}}{p_{2,2 \mid 2}}+1\right) \frac{x_{1,1 \mid 1}^{* 1}}{1+x_{1,1 \mid 1}^{* 1}}=1-\frac{p_{2,1 \mid 2}}{p_{2,2 \mid 2}} .
\end{aligned}
$$

By manipulating these two conditions, we arrive at

$$
x_{1,1 \mid 1}^{* 1}=\frac{1}{2}\left(\frac{p_{2,2 \mid 2}}{p_{2,1 \mid 2}}-1\right)<-\frac{1}{4},
$$

which is not possible.

Now assume $x_{1,1 \mid 1}^{* 1} \leq 1 / 3$. Then the following two conditions should hold:

$$
\begin{aligned}
& \left(1+\frac{p_{2,2 \mid 2}}{p_{2,1 \mid 2}}\right) \frac{1}{1+1 / 3}=2 \\
& \left(\frac{p_{2,1 \mid 2}}{p_{2,2 \mid 2}}+1\right) \frac{1 / 3}{1+1 / 3}=1-\frac{p_{2,1 \mid 2}}{p_{2,2 \mid 2}}
\end{aligned}
$$


which would imply that $p_{2,2 \mid 2} / p_{2,1 \mid 2}=5 / 3$, which contradicts the fact that $p_{2,1 \mid 2}>$ $2 p_{2,2 \mid 2}$.

Case 3

Finally, consider the case where $p_{2,1 \mid 2}=2 p_{2,2 \mid 2}$. Then the second derivative of $v$ is equal to zero and

$$
\frac{d v}{d x_{1,1 \mid 1}^{1}}=1-\frac{p_{1,1 \mid 1}}{2 p_{1,2 \mid 1}}
$$

Thus, if $p_{1,1 \mid 1} \neq 2 p_{1,2 \mid 1}$, then either $x_{1,1 \mid 1}^{* 1}=0$ or $x_{1,1 \mid 1}^{* 1}=1$, which is incompatible with equilibrium for the same reasons as before. Now assume that $p_{1,1 \mid 1}=2 p_{1,2 \mid 1}$. For household 2 it then holds that $x_{1,1 \mid 1}^{* 2}=1 / 3$. Then in equilibrium it follows that $x_{1,1 \mid 1}^{* 1}=2 / 3$. This leads to $x_{2,1 \mid 2}^{* 1}=9 / 5$ and $x_{2,2 \mid 2}^{* 1}=6 / 5$. This yields a contradiction, since the total endowment of good 2 in period 2 equals 1 . Thus, in this economy no equilibrium exists.

\section{Proof of Theorem 5.16}

We will now prove the existence of sophisticated equilibria. The next lemma states that Assumptions 1-6 and 4' suffice to obtain convex-valued demand of sophisticated households. In particular, it is shown that demand is either empty or single-valued. The first step is again to compactify the consumption sets and examine the compactified economy $\widehat{\mathcal{E}}$.

Lemma 5.40 Assume that the economy $\mathcal{E}$ satisfies Assumptions 1-6 and 4'. Consider a sophisticated household $h \in H$, a planning period $t \in T$, and a realized consumption plan $x_{-\mid t}^{h} \in X_{-\mid t}^{h}$. Then, at prices $p_{\cdot \mid t} \in P_{\cdot \mid t}, \xi_{t}^{h}\left(\cdot, x_{-\mid t}^{h}\right)$ is convex-valued and either empty or single-valued.

\section{Proof}

Since preferences are independent on past consumption, the demand correspondences will also be independent on past consumption. Therefore, the opportunity sets

$$
\begin{aligned}
\phi_{t}^{h}\left(p_{\cdot \mid t}, x_{-\mid t}^{h}\right)=\left\{x_{\cdot \mid t}^{h} \in X_{\cdot \mid t}^{h} \mid\right. & p_{\tau \mid t} x_{\tau \mid t}^{h} \leq p_{\tau \mid t} e_{\tau \mid t}^{h} \text { for all } \tau \geq t, \text { and } \\
x_{t+1, T \mid t}^{h} & \left.\in \xi_{t+1}^{h}\left(p_{t+1, T \mid t}, x_{-\mid t}^{h}, x_{t \mid t}^{h}\right)\right\}
\end{aligned}
$$

will be convex-valued. Then it is straightforward that the demand correspondences are convex-valued.

Suppose that a demand correspondence contains two elements. By convexvaluedness of the demand correspondence and by strict convexity of preferences this yields a contradiction. Thus, the demand correspondence is either empty or 
single-valued.

The next lemma shows that demand in the compactified economy satisfies standard properties needed to show existence.

Lemma 5.41 Assume that the economy $\mathcal{E}$ satisfies Assumptions 1-6, and 4'. Then, at prices $p_{\cdot \mid t} \in \widehat{P}_{\cdot \mid t}, \widehat{\xi}_{t}^{h}$ is a non-empty, compact-valued and continuous function that satisfies for every $h \in H, t \in T$ :

- Walras' law, $p_{\cdot \mid t} \square \widehat{\xi}_{t}^{h}\left(p_{\cdot \mid t}, x_{-\mid t}^{h}\right)=\left\{p_{t \mid t} e_{t \mid t}^{h}, \ldots, p_{T \mid t} e_{T \mid t}^{h}\right\}$,

- Homogeneity property, $\widehat{\xi}_{t}^{h}\left(p_{\cdot \mid t}, x_{-\mid t}^{h}\right)=\widehat{\xi}_{t}^{h}\left(\bar{p}_{|| t}, x_{-\mid t}^{h}\right)$, where for $\tau^{\prime} \geq t$, for $\lambda>0, \bar{p}_{\tau^{\prime} \mid t}=\lambda p_{\tau^{\prime} \mid t}$ and $\bar{p}_{\tau \mid t}=p_{\tau \mid t}$ for $\tau \neq \tau^{\prime}$.

\section{Proof}

The homogeneity property is straightforward.

Since in the last period the maximization problem for the sophisticated household is identical to that of the naïve household and since $\widehat{\phi}_{T}^{h}\left(p_{\cdot \mid T}, x_{-\mid T}^{h}\right)$ is independent of $x_{-\mid T}^{h}$, the characteristics of $\widehat{\xi}_{T}^{h}$ follow immediately from Lemma 5.39. By single-valuedness and upper-hemi continuity, continuity of $\widehat{\xi}_{T}^{h}$ follows immediately. We will establish the properties of the other demand correspondences by an argument of backwards induction.

Let $t \in T$. Assume that $\widehat{\xi}_{\tau}^{h}$ is non-empty, compact-valued and continuous for $\tau \in T, \tau \geq t+1$. We need to show that $\widehat{\xi}_{t}^{h}$ is non-empty, compact-valued and upper-hemi continuous. Thus, it is necessary to show that $\widehat{\phi}_{t}^{h}$ satisfies the conditions needed to apply the theorem of the maximum.

(i) Since $\widehat{\xi}_{t+1}^{h}\left(p_{\cdot \mid t+1}, x_{-\mid t}^{h}, e_{t \mid 1}^{h}\right)$ is non-empty, $p_{t \mid t} e_{t \mid 1}^{h} \leq p_{t \mid t} e_{t \mid 1}^{h}$, and $p_{\tau \mid t} x_{\tau \mid t}^{h} \leq$ $p_{\tau \mid t} e_{\tau \mid 1}^{h}, \quad \tau \geq t+1$ for $x_{t+1, T \mid t}^{h}=\widehat{\xi}_{t+1}^{h}\left(p_{\cdot \mid t+1}, x_{-\mid t}^{h}, e_{t \mid 1}^{h}\right)$, it can be seen that $\left(e_{t \mid 1}^{h}, \widehat{\xi}_{t+1}^{h}\left(p_{\cdot \mid t+1}, x_{-\mid t}^{h}, e_{t \mid 1}^{h}\right)\right) \in \widehat{\phi}_{t}^{h}\left(p_{\cdot \mid t}, x_{-\mid t}^{h}\right)$. Thus, $\widehat{\phi}_{t}^{h}\left(p_{\cdot \mid t}, x_{-\mid t}^{h}\right)$ is non-empty.

(ii) Consider the sequence $\left\{p_{\cdot \mid t}{ }^{m}\right\}_{m=1}^{\infty}$ with $p_{\cdot \mid t}{ }^{m} \rightarrow p_{\cdot \mid t}$. Let $\left\{x_{-\mid t}^{h}, x_{\cdot \mid t}^{h}\right\}_{m=1}^{m}$ be a sequence of consumption plans converging to $\left(x_{-\mid t}^{h}, x_{\cdot \mid t}^{h}\right)$, where $x_{-\mid t}^{h}{ }^{m} \in X_{-\mid t}^{h}$ and $x_{\cdot \mid t}^{h} \in \widehat{\phi}_{t}^{h}\left(p_{\cdot \mid t}{ }^{m}, x_{-\mid t}^{h}{ }^{m}\right)$ for all $m$. Then $p_{\tau \mid t}{ }^{m} x_{\tau \mid t}^{h}{ }^{m} \leq p_{\tau \mid t}{ }^{m} e_{\tau \mid t}^{h}$ for every $\tau \geq t$ and $x_{t+1, T \mid t}^{h}{ }^{m}=\widehat{\xi}_{t+1}^{h}\left(p_{t+1, T \mid t}{ }^{m}, x_{-\mid t}^{h}{ }^{m}, x_{t \mid t}^{h}{ }^{m}\right)$. By continuity it follows that $p_{\tau \mid t} x_{\tau \mid t}^{h} \leq p_{\tau \mid t} e_{\tau \mid t}^{h}$ for every $\tau \geq t$. Moreover, by continuity of $\widehat{\xi}_{t+1}^{h}, x_{t+1, T \mid t}^{h}=$ $\widehat{\xi}_{t+1}^{h}\left(p_{t+1, T \mid t}, x_{-\mid t}^{h}, x_{t \mid t}^{h}\right)$. Therefore, $x_{\cdot \mid t}^{h} \in \widehat{\phi}_{t}^{h}\left(p_{\cdot \mid t}, x_{-\mid t}^{h}\right)$. Thus, the graph of $\widehat{\phi}_{t}^{h}$ is closed.

By boundedness of $\widehat{X}_{\cdot \mid t}^{h}$ it can easily be seen, for a compact set $B$, that $\widehat{\phi}_{t}^{h}(B)$ is bounded. Therefore, $\widehat{\phi}_{t}^{h}$ is upper-hemi continuous. 
(iii) Consider a sequence $\left\{p_{\cdot \mid t}{ }^{m}, x_{-\mid t}^{h}\right\}_{m=1}^{m}$ with $\left(p_{\cdot \mid t}{ }^{m}, x_{-\mid t}^{h}{ }^{m}\right) \rightarrow\left(p_{\cdot \mid t}, x_{-\mid t}^{h}\right)$. Let $x_{\cdot \mid t}^{h} \in \widehat{\phi}_{t}^{h}\left(p_{\cdot \mid t}, x_{-\mid t}^{h}\right)$. Then, for $m$ large enough, there are $x_{t \mid t}^{h}{ }^{m} \in \widehat{X}_{t \mid t}^{h}$ such that $p_{t \mid t}{ }^{m} x_{t \mid t}^{h}{ }^{m} \leq p_{t \mid t}{ }^{m} e_{t \mid 1}^{h}$ and $x_{t \mid t}^{h}{ }^{m} \rightarrow x_{t \mid t}^{h}$.

Let $x_{t+1, T \mid t}^{h}{ }^{m}=\widehat{\xi}_{t+1}^{h}\left(p_{t+1, T \mid t}{ }^{m}, x_{-\mid t}^{h}{ }^{m}, x_{t \mid t}^{h}{ }^{m}\right)$. It follows immediately that $p_{\tau \mid t}{ }^{m} x_{\tau \mid t}^{h}{ }^{m} \leq p_{\tau \mid t}{ }^{m} e_{\tau \mid t}^{h}$ for $\tau \geq t+1$. Continuity of $\widehat{\xi}_{t+1}^{h}$ then implies that $x_{t+1, T \mid t}^{h}{ }^{m} \rightarrow \widehat{\xi}_{t+1}^{h}\left(p_{t+1, T \mid t}, x_{-\mid t}^{h}, x_{t \mid t}^{h}\right)=x_{t+1, T \mid t}^{h}$. Therefore, $x_{\cdot \mid t}^{h}{ }^{m} \in \widehat{\phi}_{t}^{h}\left(p_{\cdot \mid t}{ }^{m}, x_{-\mid t}^{h}{ }^{m}\right)$ and $x_{\cdot \mid t}^{h} \rightarrow x_{\cdot \mid t}^{h}$. Thus, $\widehat{\phi}_{t}^{h}(\cdot)$ is lower-hemi continuous.

Since $\widehat{\phi}_{t}^{h}$ is both upper-hemi and lower-hemi continuous, it is continuous.

To conclude, $\widehat{\phi}_{t}^{h}$ satisfies the conditions needed to apply the theorem of the maximum. Also, since Walras' law holds for period $t+1$, and since consumption in period $t$ does not influence the optimal consumption in period $t+1$, Walras' law holds for period $t$. The characteristics of $\widehat{\xi}_{t}^{h}$ then follow immediately.

\section{Proof of Theorem 5.16 (CONTINued)}

Note that in order to prove the existence of a sophisticated equilibrium, we can restrict ourselves to the first planning period. By similar arguments as in the foregoing section, there exists a restricted equilibrium pair $\left(p_{\cdot \mid 1}^{*}, z_{\cdot \mid 1}^{*}\right)$ such that $z_{\cdot \mid 1}^{*} \in \widehat{\zeta}_{1}\left(p_{\cdot \mid 1}^{*}\right)$ and $z_{\cdot \mid 1}^{*} \leq 0$. By monotonicity and strict convexity of preferences, it must be the case that $p_{\cdot \mid 1}^{*} \gg 0$. Therefore, and by Walras' law, it must hold that $z_{\cdot \mid 1}^{*}=0$. Denote the corresponding consumption bundles by $x_{\cdot \mid 1}^{* h}$. It remains to be shown that $x_{\cdot \mid 1}^{* h} \in \xi_{1}^{h}\left(p_{\cdot \mid 1}^{*}\right)$. Suppose that this is not the case. Then two cases can be distinguished. First assume that $x_{2, T \mid 1}^{* h} \in \xi_{2}^{h}\left(p_{2, T \mid 1}^{*}, x_{1 \mid 1}^{* h}\right)$. Then, since consumption in period 1 does not influence optimal consumption in period 2 , a similar argument as in the proof of Theorem 5.10 leads to a contradiction. Now assume that $x_{2, T \mid 1}^{* h} \notin \xi_{2}^{h}\left(p_{2, T \mid 1}^{*}, x_{1 \mid 1}^{* h}\right)$. Then, either $x_{3, T \mid 1}^{* h} \in \xi_{3}^{h}\left(p_{3, T \mid 1}^{*}, x_{1,2 \mid 1}^{* h}\right)$, which again leads to a contradiction, or $x_{3, T \mid 1}^{* h} \notin \xi_{3}^{h}\left(p_{3, T \mid 1}^{*}, x_{1,2 \mid 1}^{* h}\right)$. Continuing in this way, we end up with $x_{T \mid 1}^{* h} \notin \xi_{T}^{h}\left(p_{T \mid 1}^{*} x_{1, T-1 \mid 1}^{* h}\right)$, which leads to a contraction by the same arguments as before. Thus, a sophisticated equilibrium exists.

\section{Proof of Theorem 5.19}

Let the time-consistent allocation $x^{*}$ be Pareto efficient. Suppose that it is not MOP efficient. Then there must be an $\tilde{x}$ and a period $t^{\prime}$ such that

(i) $\sum_{h \in H} \tilde{x}_{\cdot \mid t^{\prime}}^{h}=\sum_{h \in H} e_{\cdot \mid t^{\prime}}^{h}$,

(ii) $\tilde{x}_{\cdot \mid t^{\prime}}^{h} \succeq_{x_{-\mid t^{\prime}}^{* h}} x_{\cdot \mid t^{\prime}}^{* h}$ for all $h \in H$, and

(iii) $\tilde{x}_{\cdot \mid t^{\prime}}^{h^{\prime}} \succ_{x_{-\mid t^{\prime}}^{* h^{\prime}}} x_{\cdot \mid t^{\prime}}^{* h^{\prime}}$ for some $h^{\prime} \in H$. 
But then, by time-consistency of preferences we have $\left(x_{-\mid t^{\prime}}^{* h}, \tilde{x}_{\cdot \mid t^{\prime}}^{h}\right) \succeq^{h, 1}\left(x_{-\mid t^{\prime}}^{* h}, x_{\cdot \mid t^{\prime}}^{* h}\right)$ for all $h \in H$, and $\left(x_{-\mid t^{\prime}}^{* h^{\prime}}, \tilde{x}_{\cdot \mid t^{\prime}}^{h^{\prime}}\right) \succ^{h^{\prime}, 1}\left(x_{-\mid t^{\prime}}^{* h^{\prime}}, x_{\cdot \mid t^{\prime}}^{* h^{\prime}}\right)$ for some $h^{\prime} \in H$. Then it follows from time-consistency of $x^{*}$ that $\left(x_{-\mid t^{\prime}}^{* h}, \tilde{x}_{\mid t^{\prime}}^{h}\right) \succeq^{h, 1} x_{\cdot \mid 1}^{* h}$ for all $h \in H$, and $\left(x_{-\mid t^{\prime}}^{* h^{\prime}}, \tilde{x}_{\cdot \mid t^{\prime}}^{h^{\prime}}\right) \succ^{h^{\prime}, 1} x_{\cdot \mid 1}^{* h^{\prime}}$ for some $h^{\prime} \in H$. This yields a contradiction to $x^{*}$ being Pareto efficient.

That a MOP efficient allocation is Pareto efficient, follows immediately from the definitions.

\section{Proof of Theorem 5.24}

Let $\left(p^{*}, x^{*}\right)$ be a naïve equilibrium. Suppose that $x^{*}$ is not CMP efficient, i.e. that there is a reallocation $\tilde{x}$ and a period $t^{\prime}$ that satisfy

(i) $\tilde{x}_{t^{\prime}+1, T \mid t^{\prime}}^{h}=x_{t^{\prime}+1, T \mid t^{\prime}}^{* h}$ for every $h \in H$,

(ii) $\sum_{h \in H} \tilde{x}_{t^{\prime} \mid t^{\prime}}^{h}=\sum_{h \in H} e_{t^{\prime} \mid t^{\prime}}^{h}$,

(iii) $\tilde{x}_{\cdot \mid t^{\prime}}^{h} \succeq_{x_{-\mid t^{\prime}}^{* h}} x_{\cdot \mid t^{\prime}}^{* h}$ for all $h \in H$, and

(iv) $\tilde{x}_{\cdot \mid t^{\prime}}^{h^{\prime}} \succ_{x_{-\mid t^{\prime}}^{* h^{\prime}}} x_{\cdot \mid t^{\prime}}^{* h^{\prime}}$ for some $h^{\prime} \in H$.

Then, since $\tilde{x}_{\cdot \mid t^{\prime}}^{h}$ was not chosen in equilibrium, we must have

$$
\begin{aligned}
& p_{t^{\prime} \mid t^{\prime}}^{*} \tilde{x}_{t^{\prime} \mid t^{\prime}}^{h^{\prime}}>p_{t^{\prime} \mid t^{\prime}}^{*} x_{t^{\prime} \mid t^{\prime}}^{* h^{\prime}}, \text { and } \\
& p_{t^{\prime} \mid t^{\prime}}^{*} \tilde{x}_{t^{\prime} \mid t^{\prime}}^{h} \geq p_{t^{\prime} \mid t^{\prime}}^{*} x_{t^{\prime} \mid t^{\prime}}^{* h} \text { for every household } h \in H .
\end{aligned}
$$

By summing over all households, this leads to

$$
\sum_{h \in H} p_{t^{\prime} \mid t^{\prime}}^{*} \tilde{x}_{t^{\prime} \mid t^{\prime}}^{h}>\sum_{h \in H} p_{t^{\prime} \mid t^{\prime}}^{*} x_{t^{\prime} \mid t^{\prime}}^{* h}
$$

which can be written as

$$
p_{t^{\prime} \mid t^{\prime}}^{*} \sum_{h \in H} \tilde{x}_{t^{\prime} \mid t^{\prime}}^{h}>p_{t^{\prime} \mid t^{\prime}}^{*} \sum_{h \in H} x_{t^{\prime} \mid t^{\prime}}^{* h}
$$

This leads to a contradiction, since, by assumption, we have

$$
\sum_{h \in H} \tilde{x}_{t^{\prime} \mid t^{\prime}}^{h}=\sum_{h \in H} e_{t^{\prime} \mid 1}^{h}=\sum_{h \in H} x_{t^{\prime} \mid t^{\prime}}^{* h}
$$

Thus, it follows that the naïve equilibrium allocation $x^{*}$ must be CMP efficient.

\section{Proof of Theorem 5.26}

Let $\left(p^{*}, x^{*}\right)$ be a sophisticated equilibrium. Suppose that $x^{*}$ is not CMP efficient. Then there must be a reallocation $\tilde{x}$ and a period $t^{\prime}$ that satisfy 
(i) $\tilde{x}_{t^{\prime}+1, T \mid t^{\prime}}^{h}=x_{t^{\prime}+1, T \mid t^{\prime}}^{* h}$ for every $h \in H$,

(ii) $\sum_{h \in H} \tilde{x}_{t^{\prime} \mid t^{\prime}}^{h}=\sum_{h \in H} e_{t^{\prime} \mid t^{\prime}}^{h}$,

(iii) $\tilde{x}_{\cdot \mid t^{\prime}}^{h} \succeq_{x_{-\mid t^{\prime}}^{* h}} x_{\cdot \mid t^{\prime}}^{* h}$ for all $h \in H$, and

(iv) $\tilde{x}_{\cdot \mid t^{\prime}}^{h^{\prime}} \succ_{x_{-\mid t^{\prime}}^{* h^{\prime}}} x_{\cdot \mid t^{\prime}}^{* h^{\prime}}$ for some $h^{\prime} \in H$.

Since preferences are independent of consumption in the past, optimal consumption is also not dependent on consumption in the past. Similarly, optimal future consumption is independent of current and past consumption. Therefore, the only reason why household $h^{\prime}$ has not chosen $\tilde{x}_{\cdot t^{\prime}}^{h^{\prime}}$ is that its period- $t^{\prime}$ component must be too expensive. Similarly, for every household $h$ the period- $t^{\prime}$ component of $\tilde{x}_{\cdot \mid t^{\prime}}^{h}$ must be at least as expensive as the period- $t^{\prime}$ component of $x_{\cdot \mid t^{\prime}}^{* h}$. This can be summarized as

$$
\begin{aligned}
& p_{t^{\prime} \mid t^{\prime}}^{*} \tilde{x}_{t^{\prime} \mid t^{\prime}}^{h^{\prime}}>p_{t^{\prime} \mid t^{\prime}}^{*} x_{t^{\prime} \mid t^{\prime}}^{* h^{\prime}}, \text { and } \\
& p_{t^{\prime} \mid t^{\prime}}^{*} \tilde{x}_{t^{\prime} \mid t^{\prime}}^{h} \geq p_{t^{\prime} \mid t^{\prime}}^{*} x_{t^{\prime} \mid t^{\prime}}^{* h} \text { for every household } h \in H .
\end{aligned}
$$

As in the proof of Theorem 5.24 this leads to a contradiction. It follows that the sophisticated equilibrium allocation $x^{*}$ must be CMP efficient.

Proof OF TheOREM 5.29

Let $x^{*}$ be a time-consistent allocation that is Pareto efficient. Suppose that $x^{*}$ is not $\mathrm{OP}$ efficient. Then there must be an allocation $\tilde{x}$ and a period $t^{\prime}$ such that

(i) $\sum_{h \in H} \tilde{x}_{\cdot \mid t}^{h}=\sum_{h \in H} e_{\cdot \mid t}^{h}$ for all $t \geq t^{\prime}$,

(ii) $\left(x_{-\mid t^{\prime}}^{* h}, \tilde{x}_{t^{\prime} \mid t^{\prime}}^{h}, \ldots, \tilde{x}_{t-1 \mid t-1}^{h}, \tilde{x}_{\cdot \mid t}^{h}\right) \succeq^{* h, t}\left(x_{-\mid t}^{* h}, x_{\cdot \mid t}^{* h}\right)=x_{\cdot \mid 1}^{* h}$ for all $h \in H$ and all $t \geq t^{\prime}$, and

(iii) $\left(x_{-\mid t^{\prime}}^{* h^{\prime}}, \tilde{x}_{t^{\prime} \mid t^{\prime}}^{h^{\prime}}, \ldots, \tilde{x}_{t^{\prime \prime}-1 \mid t^{\prime \prime}-1}^{h^{\prime}}, \tilde{x}_{\cdot \mid t^{\prime \prime}}^{h^{\prime}}\right) \succ^{* h^{\prime}, t^{\prime \prime}}\left(x_{-\mid t^{\prime \prime}}^{* h^{\prime}}, x_{\cdot \mid t^{\prime \prime}}^{* h^{\prime}}\right)=x_{\cdot \mid 1}^{* h^{\prime}}$ for some $h^{\prime} \in$ $H$ and some $t^{\prime \prime} \geq t^{\prime}$.

Then, by Assumption 6', we have

(ii) $\left(x_{-\mid t}^{* h}, \tilde{x}_{\cdot \mid t}^{h}\right) \succeq^{* h, t} x_{\cdot \mid 1}^{* h}$ for all $h \in H$ and all $t \geq t^{\prime}$, and

(iii) $\left(x_{-\mid t^{\prime \prime}}^{* h^{\prime}}, \tilde{x}_{\cdot \mid t^{\prime \prime}}^{h^{\prime}}\right) \succ^{* h^{\prime}, t^{\prime \prime}} x_{\cdot \mid 1}^{* h^{\prime}}$ for some $h^{\prime} \in H$ and some $t^{\prime \prime} \geq t^{\prime}$.

By time-consistency of preferences it then follows that

(ii) $\left(x_{-\mid t^{\prime \prime}}^{* h}, \tilde{x}_{\cdot \mid t^{\prime \prime}}^{h}\right) \succeq^{* h, 1} x_{\cdot \mid 1}^{* h}$ for all $h \in H$, and 
(iii) $\left(x_{-\mid t^{\prime \prime}}^{* h^{\prime}}, \tilde{x}_{\cdot \mid t^{\prime \prime}}^{h^{\prime}}\right) \succ^{* h^{\prime}, 1} x_{\cdot \mid 1}^{* h^{\prime}}$.

Since $\sum_{h \in H}\left(x_{-\mid t^{\prime \prime}}^{* h}, \tilde{x}_{\cdot \mid t^{\prime \prime}}^{h}\right)=\sum_{h \in H} e_{\cdot \mid 1}^{h}$ by definition of $x^{*}$, this would imply that $x^{*}$ is not Pareto efficient, which is a contradiction. Thus, $x^{*}$ must be OP efficient.

\section{Proof of Theorem 5.32}

Let the time-consistent allocation $x^{*}$ be Pareto efficient. Then it follows by Theorem 5.29 that $x^{*}$ is OP efficient. By the definitions it then follows immediately that $x^{*}$ is TCOP efficient.

\section{Proof of Theorem 5.33}

Let $\left(p^{*}, x^{*}\right)$ be a sophisticated equilibrium. Suppose that $x^{*}$ is not TCOP efficient. Then, there must be a time-consistent reallocation $\tilde{x}$ and a period $t^{\prime}$ that satisfy

(i) $\sum_{h \in H} \tilde{x}_{\cdot \mid 1}^{h}=\sum_{h \in H} e_{\cdot \mid 1}^{h}$,

(ii) $\left(x_{-\mid t^{\prime}}^{* h}, \tilde{x}_{\cdot \mid t^{\prime}}^{h}\right) \succeq^{* h, t} x_{\cdot \mid 1}^{* h}$ for all $h \in H$ and all $t \geq t^{\prime}$, and

(iii) $\left(x_{-\mid t^{\prime}}^{* h^{\prime}}, \tilde{x}_{\cdot \mid t^{\prime}}^{h^{\prime}}\right) \succ^{* h^{\prime}, t^{\prime \prime}} x_{\cdot \mid 1}^{* h^{\prime}}$ for some $h^{\prime} \in H$ and some $t^{\prime \prime} \geq t^{\prime}$.

If $t^{\prime \prime}=T$ then, since preferences are independent of past consumption, we must have

$$
\begin{aligned}
& p_{T \mid 1}^{*} \tilde{x}_{T \mid t^{\prime}}^{h^{\prime}}>p_{T \mid 1}^{*} x_{T \mid 1}^{* h^{\prime}}, \text { and } \\
& p_{T \mid 1}^{*} \tilde{x}_{T \mid t^{\prime}}^{h} \geq p_{T \mid 1}^{*} x_{T \mid 1}^{* h} \text { for every household } h \in H,
\end{aligned}
$$

which yields a contradiction as before. Now assume that for every household $h$ and every $\tilde{t}>t$ we have

$$
\begin{aligned}
p_{\tilde{t} \mid 1}^{*} \tilde{x}_{\tilde{t} \mid t^{\prime}}^{h} & \leq \quad p_{\tilde{t} \mid 1}^{*} x_{\tilde{t} \mid 1}^{* h}, \text { and } \\
\left(x_{-\mid t^{\prime}}^{* h}, \tilde{x}_{\cdot \mid t^{\prime}}^{h}\right) & \succeq^{* h, \tilde{t}} \quad x_{\cdot \mid 1}^{* h} .
\end{aligned}
$$

Since preferences are independent of past consumption it follows that $\tilde{x}_{\cdot \mid \tilde{t}}^{h}$ must be an optimal consumption in period $\tilde{t}$ given prices $p_{\tilde{t}, T \mid 1}^{*}$. Now assume that $t^{\prime \prime}=t$. Then we must have

$$
\begin{aligned}
& p_{t \mid 1}^{*} \tilde{x}_{t \mid t^{\prime}}^{h^{\prime}}>p_{t \mid 1}^{*} x_{t \mid 1}^{* h^{\prime}}, \text { and } \\
& p_{t \mid 1}^{*} \tilde{x}_{t \mid t^{\prime}}^{h} \geq p_{t \mid 1}^{*} x_{t \mid 1}^{* h} \text { for every household } h \in H,
\end{aligned}
$$

which again leads to a contradiction. Continuing like this we end up with this contradiction for $t=t^{\prime}$, so that case (iii) can never hold. 
It follows that the sophisticated equilibrium allocation $x^{*}$ must be TCOP efficient.

\section{ProOF OF THEOREM 5.36}

Let the time-consistent allocation $x^{*}$ be TCOP efficient. Suppose that $x^{*}$ is not $\mathrm{CP}$ efficient. Then there is an allocation $\tilde{x}$ and a period $t^{\prime}$ such that

(i) $\tilde{x}_{t^{\prime}+1, T \mid t^{\prime}}^{h}=x_{t^{\prime}+1, T \mid t^{\prime}}^{* h}=x_{t^{\prime}+1, T \mid 1}^{* h}$ for every $h \in H$,

(ii) $\sum_{h \in H} \tilde{x}_{t^{\prime} \mid t^{\prime}}^{h}=\sum_{h \in H} e_{t^{\prime} \mid t^{\prime}}^{h}$,

(iii) $\left(x_{-\mid t^{\prime}}^{* h}, \tilde{x}_{t^{\prime} \mid t^{\prime}}^{h}, x_{t^{\prime}+1 \mid t^{\prime}+1}^{* h}, \ldots, x_{t-1 \mid t-1}^{* h}, x_{\cdot \mid t}^{* h}\right) \succeq^{* h, t}\left(x_{-\mid t}^{* h}, x_{\cdot \mid t}^{* h}\right)$ for all $h \in H$ and all $t \geq t^{\prime}$, and

(iv) $\left(x_{-\mid t^{\prime}}^{* h^{\prime}}, \tilde{x}_{t^{\prime} \mid t^{\prime}}^{h^{\prime}}, x_{t^{\prime}+1 \mid t^{\prime}+1}^{* h^{\prime}}, \ldots, x_{t^{\prime \prime}-1 \mid t^{\prime \prime}-1}^{* h}, x_{\cdot \mid t^{\prime \prime}}^{* h}\right) \succ^{* h^{\prime}, t^{\prime \prime}}\left(x_{-\mid t^{\prime \prime}}^{* h^{\prime}}, x_{\cdot \mid t^{\prime \prime}}^{* h^{\prime}}\right)$ for some $h^{\prime} \in$ $H$ and some $t^{\prime \prime} \geq t^{\prime}$

which contradicts the fact that $x^{*}$ is TCOP efficient.

\section{ProOF OF THEOREM 5.38}

For sophisticated equilibria the result follows directly from Theorems 5.36 and 5.33, since sophisticated equilibrium allocations are time-consistent. Now let $\left(p^{*}, x^{*}\right)$ be a naïve equilibrium. Suppose that $x^{*}$ is not CP efficient. Then there must be a reallocation $\tilde{x}$ and a period $t^{\prime}$ that satisfy

(i) $\tilde{x}_{t^{\prime}+1, T \mid t^{\prime}}^{h}=x_{t^{\prime}+1, T \mid t^{\prime}}^{* h}$ for every $h \in H$,

(ii) $\sum_{h \in H} \tilde{x}_{t^{\prime} \mid t^{\prime}}^{h}=\sum_{h \in H} e_{t^{\prime} \mid t^{\prime}}^{h}$

(iii) $\left(x_{-\mid t^{\prime}}^{* h}, \tilde{x}_{t^{\prime} \mid t^{\prime}}^{h}, x_{t^{\prime}+1 \mid t^{\prime}+1}^{* h}, \ldots, x_{t-1 \mid t-1}^{* h}, x_{\cdot \mid t}^{* h}\right) \succeq^{* h, t}\left(x_{-\mid t}^{* h}, x_{\cdot \mid t}^{* h}\right)$ for all $h \in H$ and all $t \geq t^{\prime}$, and

(iv) $\left(x_{-\mid t^{\prime}}^{* h^{\prime}}, \tilde{x}_{t^{\prime} \mid t^{\prime}}^{h^{\prime}}, x_{t^{\prime}+1 \mid t^{\prime}+1}^{* h^{\prime}}, \ldots, x_{t^{\prime \prime}-1 \mid t^{\prime \prime}-1}^{* h}, x_{\cdot \mid t^{\prime \prime}}^{* h}\right) \succ^{* h^{\prime}, t^{\prime \prime}}\left(x_{-\mid t^{\prime \prime}}^{* h^{\prime}}, x_{\cdot \mid t^{\prime \prime}}^{* h^{\prime}}\right)$ for some $h^{\prime} \in$ $H$ and some $t^{\prime \prime} \geq t^{\prime}$.

Since preferences are independent of past consumption, $t^{\prime \prime}=t^{\prime}$. Since $\tilde{x}_{t^{\prime} \mid t^{\prime}}^{h^{\prime}}$ was not demanded in equilibrium by household $h^{\prime}$ in period $t^{\prime \prime}$, it must hold that

$$
\begin{aligned}
& p_{t^{\prime} \mid t^{\prime}}^{*} \tilde{x}_{t^{\prime} \mid t^{\prime}}^{h^{\prime}}>p_{t^{\prime} \mid t^{\prime}}^{*} x_{t^{\prime} \mid t^{\prime}}^{* h^{\prime}}, \text { and } \\
& p_{t^{\prime} \mid t^{\prime}}^{*} \tilde{x}_{t^{\prime} \mid t^{\prime}}^{h} \geq p_{t^{\prime} \mid t^{\prime}}^{*} x_{t^{\prime} \mid t^{\prime}}^{* h} \text { for every household } h \in H,
\end{aligned}
$$

which leads to a contradiction as before. Thus, $x^{*}$ must be CP efficient. 



\section{6}

\section{On the Completeness of Complete MARKETS}

This chapter ${ }^{1}$ reconsiders the allocational invariance of equilibria to different formulations of market completeness. We identify the so-far neglected assumption of sophisticated behavior as crucial to this result. The chapter studies three market structures. First, the Arrow-Debreu setting is considered, where markets do not reopen in the future. Second, sequentially complete markets are analyzed, where goods on the spot markets and all contingent one-period ahead commodities can be traded in every state. Finally, complete markets are analyzed, where all possible contingent commodities can be traded at every state. Preferences may be time-consistent or time-inconsistent. A distinction is made between naïve and sophisticated behavior. Whereas naïve households do not realize that their preferences might change in the future, sophisticated households do realize this and only make plans for the future that they expect to carry out in the future. For economies with time-inconsistent preferences, Arrow-Debreu equilibria are not related to either sequentially complete equilibria or complete equilibria. It does hold that every equilibrium consumption that can be attained in sequentially complete markets, can also be attained in complete markets. An example shows that the converse is not true for naïve economies. Finally, when preferences are restricted to be time-consistent and households are sophisticated, the three market structures yield the same equilibrium consumption. Surprisingly, for naïve households, this result is not true, even when preferences are time-consistent.

\subsection{Introduction}

Debreu (1959) discusses how the one-period Arrow-Debreu (1954) model can be used to analyze multi-period settings with uncertainty. The crucial idea is to in-

\footnotetext{
${ }^{1}$ This chapter is based on Herings and Rohde (2005).
} 
clude the period and the state of the world at the time of delivery in the definition of a commodity, resulting in contingent commodities. Debreu assumes that markets are complete in the sense that all contingent commodities can be traded in the first period and does not allow for markets to reopen after the first period.

As an alternative to the assumption that all contingent commodities can be traded in the initial period, sequentially complete markets have been considered. In a sequentially complete market structure, it is typically assumed that at every state there are complete spot markets for commodities available at that state together with a restricted set of markets for commodities available at future states. It is well-known that, under certain assumptions, equilibria in the Arrow-Debreu model are allocationally equivalent to equilibria in sequentially complete models, see Arrow (1953). Donaldson and Selden (1981) and Haller (1990) provide two discussions of the assumptions needed for this result. Drèze and Herings (2003) show in an example that there might be equilibrium continuations in a sequentially complete markets setting that are not allocationally equivalent to any equilibrium in the Arrow-Debreu setting. A closer look at their example suggests that the result is driven by the fact that in the Arrow-Debreu setting, markets are not reopened. Chattopadhyay and Gottardi (1999) consider differences between complete and sequentially complete market structures in overlapping generations models.

This chapter studies multi-period economies subject to uncertainty, where markets may reopen at future states. At every state, households are endowed with preferences that may or may not be time-consistent. In general equilibrium models, time-inconsistent preferences have been introduced by Luttmer and Mariotti (2003) and Herings and Rohde (2006). We consider three market structures that are commonly believed to result in the same set of competitive equilibria. First, we consider the Arrow-Debreu (AD) structure with complete markets in the first period and no reopening of markets as time passes. Second, we consider sequentially complete (SC) markets, where in every state, people can trade the goods to be consumed in that state and all one-period ahead contingent commodities. Finally, we consider the complete $(\mathrm{C})$ market structure. We say that markets are complete if, in every state, there are complete markets for all contingent commodities to be consumed in that state or in a later state. Figure 6.1 indicates which markets are open at various periods under these three market structures in an economy with four periods.

We follow Pollak (1968) in making a distinction between naïve and sophisticated behavior. Whereas naïve households believe that their preferences will not change in the future, sophisticated households correctly anticipate their future preferences. The latter households make plans that they expect to stick to in 

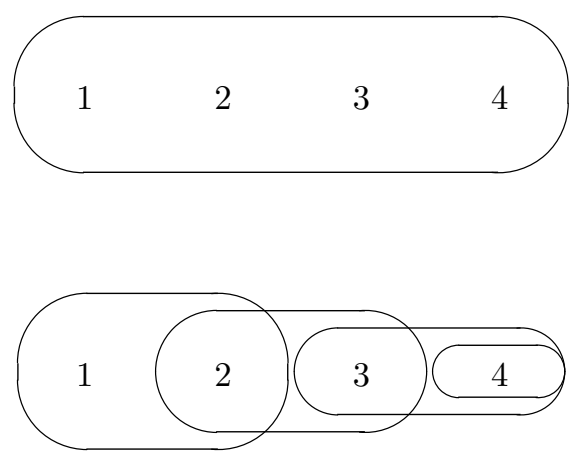

SC

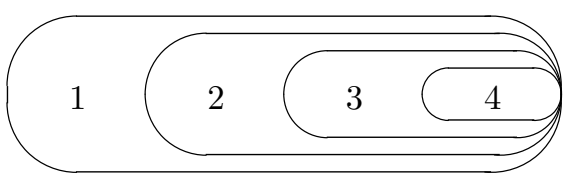

C

Figure 6.1: Commodities traded simultaneously in a 4-period economy

the future. Sophisticated households can be interpreted as consisting of multiple agents, playing a subgame perfect equilibrium against themselves. We will analyze these two types of behavior for both time-consistent and time-inconsistent preferences.

In general, the Arrow-Debreu market structure leads to equilibrium allocations that differ from equilibrium allocations corresponding to the other two market structures. The explanation is that the Arrow-Debreu market structure yields the commitment power needed not to re-trade. For sophisticated economies, sequentially complete markets yield the same allocations as complete markets do, even when preferences are allowed to be time-inconsistent. The assumption of sophisticated behavior is extremely demanding. It might be more reasonable to assume that households, at least partly, mispredict their future preferences (Loewenstein, O’Donoghue, and Rabin (2003)). For naïve economies any sequentially complete equilibrium is allocationally equivalent to some complete equilibrium, but the converse statement does not hold.

When preferences are time-consistent, we show that all three markets structures are allocationally equivalent for sophisticated economies. For naïve economies, 
every equilibrium in the Arrow-Debreu market structure is allocationally equivalent to some sequentially complete equilibrium and some complete equilibrium, but surprisingly the reverse does not hold. The results of this chapter are summarized in Figures 6.2, 6.3, 6.4 and 6.5. In those figures, an arrow from 'A' to 'B' indicates that any concept ' $\mathrm{A}$ ' equilibrium allocation is allocationally equivalent to some concept ' $\mathrm{B}$ ' equilibrium allocation.

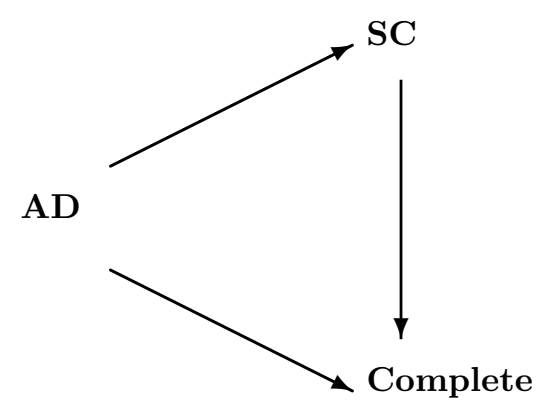

Figure 6.2: Naïve equilibria when preferences are time-consistent

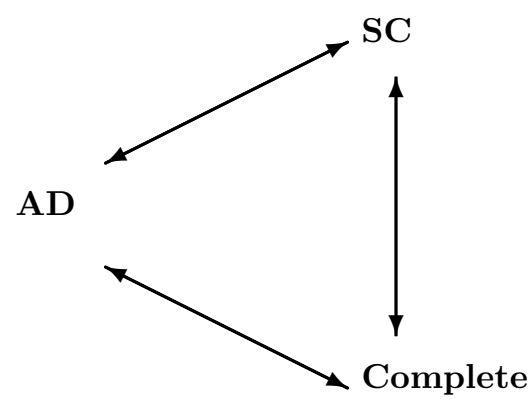

Figure 6.3: Sophisticated equilibria when preferences are time-consistent

This chapter therefore identifies a crucial assumption that has to be satisfied in order for complete and sequentially complete markets to be equivalent: behavior should be sophisticated. For equivalence to Arrow-Debreu markets, it is also needed that preferences are time-consistent.

Section 6.2 starts by describing the primitives of the economies as considered in this chapter, without elaborating on market structures. Section 6.3 describes the Arrow-Debreu market structure. Sections 6.4 and 6.5 analyze the sequentially complete markets structure and the complete one, respectively. Both these 
$\mathrm{AD}$

$\mathrm{SC}$

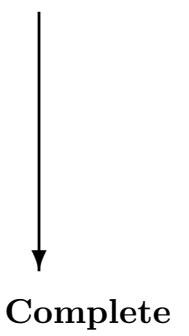

Figure 6.4: Naïve equilibria when preferences are time-inconsistent

$\mathrm{AD}$

$\mathrm{SC}$

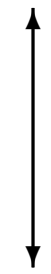

\section{Complete}

Figure 6.5: Sophisticated equilibria when preferences are time-inconsistent

sections are built up in the same fashion. First, naïve and sophisticated behavior is described. Then, properties of the specific market structure are studied. Section 6.6 then concludes. All proofs are in the Appendix.

\subsection{The Primitives of the Economy}

There is a finite number of periods, denoted by the integer $T^{2}$. In every period $t \in\{2, \ldots, T\}$, exactly one event in the finite set $\Omega$ occurs. A sequence of $T-1$ events constitutes a state. We let $S=\Omega^{T-1}$ be the set of states. For every $s \in S$ and every $t \in\{2, \ldots, T\}$, we define the state at period $t$ by

$$
s(t)=\left\{\left(\omega_{\tau}\right)_{\tau=2}^{t} \in \Omega^{t-1} \mid \exists\left(\omega_{\tau}\right)_{\tau=t+1}^{T} \in \Omega^{T-t} \text { with }\left(\omega_{\tau}\right)_{\tau=2}^{T}=s\right\} .
$$

\footnotetext{
${ }^{2}$ As no confusion will arise, for finite sets we will use the same notation to indicate the set and its cardinality.
} 
For $t=1$, we define $s(1)=1$.

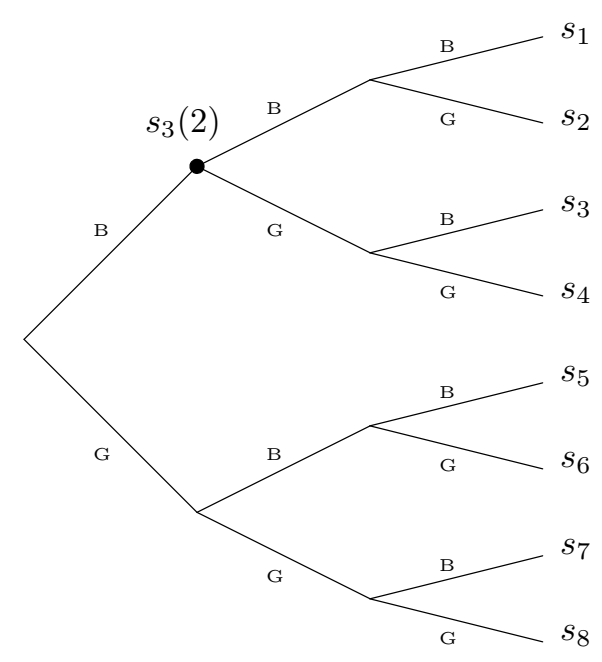

Figure 6.6: An economy with $T=4$ and $\Omega=\{\operatorname{Bad}$, Good $\}$

Figure 6.6 illustrates the structure of an economy with 4 periods $(T=4)$ and two events in every period $(\Omega=\{\operatorname{Bad}, \operatorname{Good}\})$. A state in period 4 is a path from the root of the tree to an end-node. Thus, in the figure there are 8 states in period 4. Consider the state that corresponds to the path that leads to the third end-node from above. We call this state $s_{3}=(B, G, B)$. We have $s_{3}(2)=B$, $s_{3}(3)=(B, G)$, and $s_{3}(4)=(B, G, B)$. For every $s(t)$ we define $s^{+}(t)=\left\{s^{\prime}(t+1) \in\right.$ $\left.\Omega^{t} \mid s^{\prime} \in S, s^{\prime}(t)=s(t)\right\}$.

The economy consists of a finite number of households $h \in H$. In every state of every period, households consume a finite number of goods $l \in L$. For $s \in S$ and $\tau \geq t$, the consumption bundle $x_{s(\tau) \mid s(t)}^{h}$ denotes the consumption of every good at state $s(\tau)$ anticipated in state $s(t)$. Consumption for state $s(t)$ and every later state anticipated at $s(t)$, is given by the consumption plan $x_{\cdot \mid s(t)}^{h}$. Consumption for state $s(\tau)$ and all states that can be reached from there up till period $\tau^{\prime}$, as anticipated in state $s(t)$, is given by $x_{s(\tau), \tau^{\prime} \mid s(t)}^{h}$. By $x_{\cdot \mid s(t)}^{h} \prec \hat{x}_{\cdot \mid s(\tau)}^{h}$, we denote the consumption $x_{\cdot \mid s(t)}^{h}$ with $x_{s(\tau), T \mid s(t)}^{h}$ replaced by $\hat{x}_{\cdot \mid s(\tau)}^{h}$. For $t \in T$, we define $\mathcal{L}_{t}=L \sum_{\tau=1}^{T-t} \Omega^{\tau}$, the total number of commodities to be consumed at states later than $s(t)$. Consumption in every state is limited to the consumption sets $X_{\cdot \mid s(t)}^{h} \subset \mathbb{R}^{L+\mathcal{L}_{t}}$, where the interpretation of sub- and superscripts is similar as 
before. A subscript $l$ is added to denote consumption of good $l$. In every state $s(t)$, households have initial endowments $e_{\cdot \mid s(t)}^{h}$. Households are assumed to have correct expectations about consumption sets and endowments.

In every state, households have preferences over consumption in that state and all possible future states. Preferences of household $h$ in state $s(t)$ are represented by $\succeq^{h, s(t)}$, a relation defined on $X_{\cdot \mid s(t)}^{h}$. The preferences of household $h$ are denoted by $\succeq^{h}=\left(\succeq^{h, s(t)}\right)_{s \in S, t \in T}$.

Preferences might depend on the state of nature and can change over time. Consider a consumption plan in state $s(t)$. If we replace consumption from a future state $s(\tau)$ on by consumption that will be preferred once state $s(\tau)$ is reached, then time-consistency of preferences implies that the resulting consumption plan at state $s(t)$ is preferred to the original one.

\section{Definition 6.1 Time-consistent Preferences}

Preferences for household $h$ are time-consistent if for all states $s \in S$, for all periods $t, \tau$ with $\tau \geq t$, for every $x_{\cdot \mid s(t)}^{h} \in X_{\cdot \mid s(t)}^{h}$ and every $\widehat{x}_{\cdot \mid s(\tau)}^{h} \in X_{\cdot \mid s(\tau)}^{h}$, we have

$$
x_{\cdot \mid s(t)}^{h} \succeq^{h, s(t)} \quad x_{\cdot \mid s(t)}^{h}\left\langle\widehat{x}_{\cdot \mid s(\tau)}^{h}\right.
$$

if and only if

$$
x_{s(\tau), T \mid s(t)}^{h} \succeq^{h, s(\tau)} \quad \widehat{x}_{\cdot \mid s(\tau)}^{h} .
$$

Preferences are time-inconsistent if they are not time-consistent.

Preferences of a household in a particular state $s(t)$ are locally non-satiated in state $s\left(t^{\prime}\right)$, where $t^{\prime} \geq t$, if for every consumption plan in state $s(t)$, we can find another consumption plan in state $s(t)$ that (i) only deviates from the initial consumption plan in state $s\left(t^{\prime}\right)$, (ii) is arbitrarily close to the initial consumption plan, and (iii) is preferred to the initial consumption plan. This can be formalized as follows. Consider a household $h$, a state $s$, and two periods $t, t^{\prime}$ with $t^{\prime} \geq t$. Preferences $\succeq^{h, s(t)}$ are locally non-satiated in $s\left(t^{\prime}\right)$ if for every $x_{\cdot \mid s(t)}^{h} \in X_{\cdot \mid s(t)}^{h}$, for every $\varepsilon>0$, there is an $\bar{x}_{\cdot \mid s(t)}^{h} \in X_{\cdot \mid s(t)}^{h}$ with $\bar{x}_{s^{\prime}(\tau) \mid s(t)}^{h}=x_{s^{\prime}(\tau) \mid s(t)}^{h}$ for every $s^{\prime}(\tau) \neq s\left(t^{\prime}\right),\left\|\bar{x}_{\cdot \mid s(t)}^{h}-x_{\cdot \mid s(t)}^{h}\right\|<\varepsilon$ and $\bar{x}_{\cdot \mid s(t)}^{h} \succ^{h, s(t)} x_{\cdot \mid s(t)}^{h}$. An economy with preferences $\left(\succeq^{h, s(t)}\right)_{h \in H, s \in S, t \in T}$ is locally non-satiated if for every state $s$ and every $t, t^{\prime}$ with $t^{\prime} \geq t$ there is a household $h$ such that $\succeq^{h, s(t)}$ is locally non-satiated in $s\left(t^{\prime}\right)$.

An economy $\mathcal{E}$ is described by its primitives $\left(X_{\cdot \mid 1}^{h}, e_{\cdot \mid 1}^{h}, \succeq^{h}\right)_{h \in H}$. A household will sell its endowments and use the revenues from this sale to buy the goods the household desires most. The timing of the opportunities to sell endowments will depend on the market structure. 


\subsection{Arrow-Debreu Markets}

This section considers the model of Arrow and Debreu (1954) and Debreu (1959). There are complete markets in contingent commodities in period 1. Markets do not reopen in later periods. All commodities can be traded in period 1 against the prices $p_{\cdot \mid 1}$. Prices for commodities in state $s(t)$ are then given by $p_{s(t) \mid 1}$. Prices for state $s(t)$ and all states that can be reached from $s(t)$ are denoted by $p_{s(t), T \mid 1}$. We denote the set of possible prices by $P_{\cdot \mid 1}=\mathbb{R}^{L+\mathcal{L}_{1}}$.

In the Arrow-Debreu model, markets do not reopen after period 1. Therefore, it is implicitly assumed that consumption in those later periods is completely determined by the choices made in period 1. Moreover, since markets do not reopen after period 1 , there is no need to specify prices in periods after period 1 . To be consistent in notation throughout this chapter, we do want to specify those prices. Without loss of generality, we set prices in states later than period 1 equal to the prices in period 1 of corresponding contingent commodities. We define $p_{\cdot \mid s(t)}=p_{s(t), T \mid 1}$ for every $s(t)$, and $P_{\cdot \mid s(t)}=\mathbb{R}^{L+\mathcal{L}_{t}}$ and $P=\prod_{s(t)} P_{\cdot \mid s(t)}$.

The opportunity set of household $h$ in period 1 is given by

$$
\breve{\gamma}_{1}^{h}\left(p_{\cdot \mid 1}, e_{\cdot \mid 1}^{h}\right)=\left\{x_{\cdot \mid 1}^{h} \in X_{\cdot \mid 1}^{h} \mid p_{\cdot \mid 1} x_{\cdot \mid 1}^{h} \leq p_{\cdot \mid 1} e_{\cdot \mid 1}^{h}\right\}
$$

The opportunity set of household $h$ in any state $s(t)$ with $t>1$ is given by

$$
\breve{\gamma}_{s(t)}^{h}\left(p_{\cdot \mid s(t)}, x_{s(t), T \mid s(t-1)}^{h}\right)=\left\{x_{s(t), T \mid s(t-1)}^{h}\right\} .
$$

A household selects a most preferred consumption plan in the opportunity set. Thus, the demand sets are given by

$$
\begin{aligned}
\breve{\delta}_{s(t)}^{h}\left(p_{\cdot \mid s(t)}, x_{s(t), T \mid s(t-1)}^{h}\right) & =\left\{\bar{x}_{\cdot \mid s(t)}^{h} \in \breve{\gamma}_{s(t)}^{h}\left(p_{\cdot \mid s(t)}, x_{s(t), T \mid s(t-1)}^{h}\right) \mid\right. \\
\nexists x_{\cdot \mid s(t)}^{h} & \left.\in \breve{\gamma}_{s(t)}^{h}\left(p_{\cdot \mid s(t)}, x_{s(t), T \mid s(t-1)}^{h}\right) \text { with } x_{\cdot \mid s(t)}^{h} \succ^{h, s(t)} \bar{x}_{\cdot \mid s(t)}^{h}\right\} .
\end{aligned}
$$

In equilibrium, total demand must equal total endowments. For notational purposes, we define $x_{1, T \mid s(0)}^{h}=e_{\cdot \mid 1}^{h}$.

\section{Definition 6.2 Arrow-Debreu (AD) Equilibrium}

A pair $\left(p^{*}, x^{*}\right) \in P \times X$ is an Arrow-Debreu $(A D)$ equilibrium of the economy $\mathcal{E}$ if

(a) $x_{\cdot \mid s(t)}^{* h} \in \breve{\delta}_{s(t)}^{h}\left(p_{\cdot \mid s(t)}^{*}, x_{s(t), T \mid s(t-1)}^{* h}\right)$ for all $h \in H, t \in T, s \in S$,

(b) $\sum_{h \in H} x_{\cdot \mid s(t)}^{* h}=\sum_{h \in H} e_{\cdot \mid s(t)}^{h}$ for all $t \in T, s \in S$,

(c) $p_{\cdot \mid s(t)}^{*}=p_{s(t), T \mid 1}^{*}$ for all $t \in T, s \in S$. 


\subsection{Sequentially Complete Markets}

This section considers sequentially complete markets. In every state there are spot markets and forward markets for one-period ahead contingent commodities. In period $t$ households can trade all goods for period $t$ and all goods for all possible events in period $t+1$. They have to form expectations about prices for goods that will be consumed in later periods, i.e. periods from $t+2$ on. We assume that they form point expectations and that all households form the same expectations. We let $P \cdot \mid s(t)=\mathbb{R}^{L+\mathcal{L}_{t}}, \bar{Q}_{\cdot \mid s(t)}=\mathbb{R}^{\mathcal{L}_{t}}, P=\prod_{s(t)} P_{\cdot \mid s(t)}$ and $\bar{Q}=\prod_{s(t)} \bar{Q}_{\cdot \mid s(t)}$. Prices and expected (at state $s(t)$ ) prices on spot markets will be denoted by $p_{\cdot \mid s(t)} \in$ $P_{. \mid s(t)}$. Prices and expected (at state $s(t)$ ) prices of forward commodities will be denoted by $q_{\cdot \mid s(t)} \in \bar{Q}_{\cdot \mid s(t)}$. The (expected) prices in state $s(t)$ of commodities to be delivered in state $s(\tau)$ and traded in $s(\tau-1)$, are denoted by $q_{s(\tau) \mid s(t)}$. We define $\bar{Y}_{\cdot \mid s(t)}^{h}=\mathbb{R}^{\mathcal{L}_{t}}$ and $\bar{Y}=\prod_{h \in H} \prod_{s(t)} \bar{Y}_{\cdot \mid s(t)}^{h}$. For household $h$ the forward commodity bundle for state $s(\tau)$ that is expected in state $s(t)$ to be bought in state $s(\tau-1)$ is denoted by $y_{s(\tau) \mid s(t)}^{h}$. For notational purposes, we define $y_{\cdot \mid s(0)}^{h}=e_{\cdot \mid 1}^{h}$ and $y_{s(t) \mid s(t)}^{h}=y_{s(t) \mid s(t-1)}^{h}$.

The structure of sequentially complete markets is illustrated in Figure 6.7. There, rows refer to the period in which the commodities are bought and columns refer to the period in which the commodities are delivered. The first block of 4 rows refers to the perspective of a household in period 1. The next blocks are the perspectives of the households in the next periods. Bullets indicate that the prices and consumption are directly observable in the markets, open bullets denote expectations.

When markets can reopen in the future, and preferences are allowed to be time-inconsistent, a conflict can arise between the preferences from the perspective of one state and those of another, later, state. Following Pollak (1968), we make a distinction between naïve and sophisticated households. Naïve households will typically revise their plans over time. They have the incorrect belief that their preferences will not change in the future. Sophisticated households correctly anticipate their future preferences. They only make choices that they expect to stick to in the future. A more realistic assumption would be that households are partly sophisticated and incorrectly anticipate future preferences. For these households the relation between the various market structures will be similar to those for naïve households. Therefore, we restrict attention to naïve and (fully) sophisticated households. First, we consider naïve and then sophisticated behavior. 


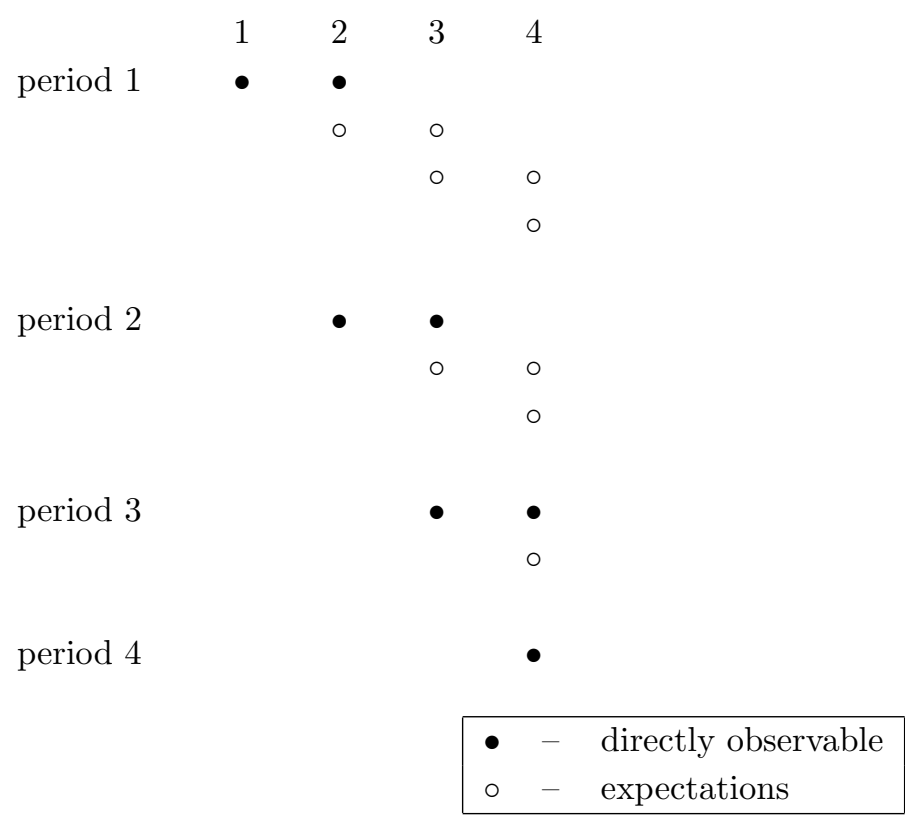

Figure 6.7: Structure of sequentially complete markets

\subsubsection{Naïve Behavior}

In every state, naïve households sell the endowments of that particular state and the one-period ahead endowments. They use the revenue from selling these commodities to buy goods on the spot markets and to buy one-period ahead contingent commodities. At each state $s(t)$ households form expectations about all future prices. We assume that naïve households have rational price expectations. By this we mean that in every period and every state they have common point expectations about prices and that prices are expected to be market clearing. Thus, rational price expectations are correct price expectations (Radner, 1972) given the belief that preferences will not change in the future. Nevertheless, preferences can change and that will induce new rational price expectations in the future, which will again be correct from the viewpoint of that future state.

The opportunity set of household $h$ in state $s(t)$ is given by

$$
n \bar{\gamma}_{s(t)}^{h}\left(p_{\cdot \mid s(t)}, q \cdot \mid s(t), y_{s(t) \mid s(t-1)}^{h}, e_{t+1, T \mid s(t)}^{h}\right)=\left\{\left(x_{\cdot \mid s(t)}^{h}, y_{\cdot \mid s(t)}^{h}\right) \in X_{\cdot \mid s(t)}^{h} \times \bar{Y}_{\cdot \mid s(t)}^{h} \mid\right.
$$




$$
\begin{aligned}
p_{s^{\prime}(\tau) \mid s(t)} x_{s^{\prime}(\tau) \mid s(t)}^{h}+\sum_{s^{\prime}(\tau+1) \in s^{\prime}+(\tau)} q_{s^{\prime}(\tau+1) \mid s(t)} y_{s^{\prime}(\tau+1) \mid s(t)}^{h} \leq \\
p_{s^{\prime}(\tau) \mid s(t)} y_{s^{\prime}(\tau) \mid s(t)}^{h}+\sum_{s^{\prime}(\tau+1) \in s^{\prime}(\tau)} q_{s^{\prime}(\tau+1) \mid s(t)} e_{s^{\prime}(\tau+1) \mid s(t)}^{h}
\end{aligned}
$$

for every $\tau \geq t$, for every $s^{\prime}(\tau)$ with $\left.s^{\prime}(t)=s(t)\right\}$,

where, by definition, $y_{s(t) \mid s(t)}^{h}=y_{s(t) \mid s(t-1)}^{h}$ and $y_{1 \mid s(0)}^{h}=e_{1 \mid 1}^{h}$.

The demand set of household $h$ in state $s(t)$ is then given by

$$
\begin{aligned}
& { }^{n} \bar{\delta}_{s(t)}^{h}(p \cdot \mid s(t) \\
& \quad\left\{q_{\cdot \mid s(t)}, y_{s(t) \mid s(t-1)}^{h}, e_{t+1, T \mid s(t)}^{h}\right)= \\
& \quad\left(\bar{x}_{\cdot \mid s(t)}^{h}, \bar{y}_{\cdot \mid s(t)}^{h}\right) \in{ }^{n} \bar{\gamma}_{s(t)}^{h}\left(p_{\cdot \mid s(t)}, q \cdot \mid s(t), y_{s(t) \mid s(t-1)}^{h}, e_{t+1, T \mid s(t)}^{h}\right) \mid \\
& \quad \nexists\left(x_{\cdot \mid s(t)}^{h}, y_{\cdot \mid s(t)}^{h}\right) \in{ }^{n} \bar{\gamma}_{s(t)}^{h}\left(p_{\cdot \mid s(t)}, q \cdot \mid s(t), y_{s(t) \mid s(t-1)}^{h}, e_{t+1, T \mid s(t)}^{h}\right) \\
& \left.\quad \text { with } x_{\cdot \mid s(t)}^{h} \succ^{h, s(t)} \bar{x}_{\cdot \mid s(t)}^{h}\right\} .
\end{aligned}
$$

Prices and consumption are said to constitute a naïve sequentially complete equilibrium if consumption is in the demand sets and if markets clear and are expected to clear in every state.

\section{Definition 6.3 Naïve Sequentially Complete (SC) Equilibrium}

A pair $\left(p^{*}, q^{*}, x^{*}, y^{*}\right) \in P \times \bar{Q} \times X \times \bar{Y}$ is a Naïve Sequentially Complete (SC) equilibrium of the economy $\mathcal{E}$ if

(a) $\left(x_{\cdot \mid s(t)}^{* h}, y_{\cdot \mid s(t)}^{* h}\right) \in{ }^{n} \bar{\delta}_{s(t)}^{h}\left(p_{\cdot \mid s(t)}^{*}, q_{\cdot \mid s(t)}^{*}, y_{s(t) \mid s(t-1)}^{* h}, e_{t+1, T \mid s(t)}^{h}\right)$ for all $h \in H, t \in T$, $s \in S$,

(b) $\sum_{h \in H} x_{\cdot \mid s(t)}^{* h}=\sum_{h \in H} e_{\cdot \mid s(t)}^{h}$ for all $t \in T, s \in S$, and

(c) $\sum_{h \in H} y_{\cdot \mid s(t)}^{* h}=\sum_{h \in H} e_{t+1, T \mid s(t)}^{h}$ for all $t \in T, s \in S$.

\subsubsection{Sophisticated Behavior}

Sophisticated households know exactly how their preferences will change in the future. Therefore, they can correctly predict their future behavior. Moreover, they only make plans that they expect to adhere to. They face the additional constraint that their expected future consumption should be in their future demand sets given expected future prices.

The opportunity set of sophisticated household $h$ in state $s(T)$ is the same as for its naïve counterpart:

$$
{ }^{s} \bar{\gamma}_{s(T)}^{h}\left(p_{\cdot \mid s(T)}, y_{s(T) \mid s(T-1)}^{h}\right)={ }^{n} \bar{\gamma}_{s(T)}^{h}\left(p_{\cdot \mid s(T)}, y_{s(T) \mid s(T-1)}^{h}\right) .
$$


The opportunity set of sophisticated household $h$ in state $s(t)$, where $t<T$, is given by

$$
\begin{gathered}
s \bar{\gamma}_{s(t)}^{h}\left(p_{\cdot \mid s(t)}, q \cdot \mid s(t)\right. \\
\left.y_{s(t) \mid s(t-1)}^{h}, e_{t+1, T \mid s(t)}^{h}\right)=\left\{\left(x_{\cdot \mid s(t)}^{h}, y_{\cdot \mid s(t)}^{h}\right) \in X_{\cdot \mid s(t)}^{h} \times \bar{Y}_{\cdot \mid s(t)}^{h} \mid\right. \\
(i) p_{s^{\prime}(\tau) \mid s(t)} x_{s^{\prime}(\tau) \mid s(t)}^{h}+\sum_{s^{\prime}(\tau+1) \in s^{\prime+}(\tau)} q_{s^{\prime}(\tau+1) \mid s(t)} y_{s^{\prime}(\tau+1) \mid s(t)}^{h} \leq \\
p_{s^{\prime}(\tau) \mid s(t)} y_{s^{\prime}(\tau) \mid s(t)}^{h}+\sum_{s^{\prime}(\tau+1) \in s^{\prime+}(\tau)} q_{s^{\prime}(\tau+1) \mid s(t)} e_{s^{\prime}(\tau+1) \mid s(t)}^{h}
\end{gathered}
$$

for every $\tau \geq t$, for every $s^{\prime}(\tau)$ with $s^{\prime}(t)=s(t)$,

and

$$
\begin{aligned}
(i i)\left(x_{s(t+1), T \mid s(t)}^{h}, y_{s^{+}(t+1), T \mid s(t)}^{h}\right) \in & \\
& s \bar{\delta}_{s(t+1)}^{h}\left(p_{s(t+1), T \mid s(t)}, q_{s^{+}(t+1), T \mid s(t)}, y_{s(t+1) \mid s(t)}^{h}, e_{t+2, T \mid s(t+1)}^{h}\right) \\
& \text { for every } \left.s(t+1) \in s^{+}(t)\right\} .
\end{aligned}
$$

The demand set of a sophisticated household is given by

$$
\begin{aligned}
& s \bar{\delta}_{s(t)}^{h}\left(p_{\cdot \mid s(t)}, q_{\cdot \mid s(t)}, y_{s(t) \mid s(t-1)}^{h}, e_{t+1, T \mid s(t)}^{h}\right)= \\
& \left\{\left(\bar{x}_{\cdot \mid s(t)}^{h}, \bar{y}_{\cdot \mid s(t)}^{h}\right) \in{ }^{s} \bar{\gamma}_{s(t)}^{h}\left(p_{\cdot \mid s(t)}, q \cdot \mid s(t), y_{s(t) \mid s(t-1)}^{h}, e_{t+1, T \mid s(t)}^{h}\right) \mid\right. \\
& \nexists\left(x_{\cdot \mid s(t)}^{h}, y_{\cdot \mid s(t)}^{h}\right) \in{ }^{s} \bar{\gamma}_{s(t)}^{h}\left(p_{\cdot \mid s(t)}, q_{\cdot \mid s(t)}, y_{s(t) \mid s(t-1)}^{h}, e_{t+1, T \mid s(t)}^{h}\right) \\
& \text { with } \left.x_{\cdot \mid s(t)}^{h} \succ^{h, s(t)} \bar{x}_{\cdot \mid s(t)}^{h}\right\} .
\end{aligned}
$$

Prices, consumption bundles and purchases of forward commodities constitute a sophisticated sequentially complete equilibrium if actual and planned supply of spot and forward commodities equals actual and planned demand, price expectations are correct, and expected consumption and expected purchases of forward commodities equal realized consumption and realized purchases of forward commodities. This is formalized in the following definition.

\section{Definition 6.4 Sophisticated Sequentially Complete (SC) Equilibrium} A pair $\left(p^{*}, q^{*}, x^{*}, y^{*}\right) \in P \times \bar{Q} \times X \times \bar{Y}$ is a Sophisticated Sequentially Complete $(S C)$ equilibrium of the economy $\mathcal{E}$ if

(a) $\left(x_{\cdot \mid s(t)}^{* h}, y_{\cdot \mid s(t)}^{* h}\right) \in{ }^{s} \bar{\delta}_{s(t)}^{h}\left(p_{\cdot \mid s(t)}^{*}, q_{\cdot \mid s(t)}^{*}, y_{s(t) \mid s(t-1)}^{* h}, e_{t+1, T \mid s(t)}^{h}\right)$ for all $h \in H, t \in T$, $s \in S$, 
(b) $\sum_{h \in H} x_{\cdot \mid s(t)}^{* h}=\sum_{h \in H} e_{\cdot \mid s(t)}^{h}$ for all $t \in T, s \in S$,

(c) $\sum_{h \in H} y_{\cdot \mid s(t)}^{* h}=\sum_{h \in H} e_{t+1, T \mid s(t)}^{h}$ for all $t \in T, s \in S$,

(d) $p_{s(\tau), T \mid s(t)}^{*}=p_{\cdot \mid s(\tau)}^{*}$ for all $s \in S, t, \tau \in T$ with $t \leq \tau$,

(e) $q_{s(\tau), T \mid s(t)}^{*}=q_{s(\tau), T \mid s(\tau-1)}^{*}$ for all $s \in S, t, \tau \in T$ with $t<\tau$,

(f) $x_{s(\tau), T \mid s(t)}^{* h}=x_{\cdot \mid s(\tau)}^{* h}$ for all $h \in H, s \in S, t, \tau \in T$ with $t \leq \tau$, and

(g) $y_{s(\tau), T \mid s(t)}^{* h}=y_{s(\tau), T \mid s(\tau-1)}^{* h}$ for all $h \in H, s \in S, t, \tau \in T$ with $t<\tau$.

\subsubsection{Properties of Sequentially Complete Equilibria}

In both naïve SC and sophisticated SC equilibria, expectations of relative prices on spot markets are equal to the corresponding expected relative prices of assets or one-period ahead contingent commodities, except when all those asset prices are equal to zero. This is formalized in the following theorem. Svensson (1976) derives a similar result in a two-period economy without uncertainty. He called these price expectations stationary point expectations.

Theorem 6.5 Let the economy $\mathcal{E}$ be locally non-satiated and let $\left(p^{*}, q^{*}, x^{*}, y^{*}\right)$ be a naive $S C$ equilibrium of $\mathcal{E}$. Then for every $s, s^{\prime} \in S$ and every $t, \tau \in T$ with $\tau>t$ and $s^{\prime}(t)=s(t)$ there is $\mu_{s^{\prime}(\tau) \mid s(t)} \in \mathbb{R}$ such that

$$
q_{s^{\prime}(\tau) \mid s(t)}^{*}=\mu_{s^{\prime}(\tau) \mid s(t)} p_{s^{\prime}(\tau) \mid s(t)}^{*} .
$$

The result of Theorem 6.5 is driven by the arbitrage opportunities that are created in locally non-satiated economies if the condition of the result would be violated. This intuition is entirely correct for the naïve case. The sophisticated case is more difficult. The reason is that a sophisticated household cannot guarantee that his future self will behave as he would like him to behave. Therefore, changing income in a future state may make the future self consume a bundle that the current self does not like at all. The availability of an arbitrage opportunity in a particular state is not sufficient to drive the result. The arbitrage opportunity needs to be such that also income in future states is unchanged, so that future selves will behave the same, irrespective of whether the arbitrage opportunity is taken or not. Our proof for the naïve case is constructed in such a way that these properties are satisfied, and therefore applies to the sophisticated case as well.

Theorem 6.6 Let the economy $\mathcal{E}$ be locally non-satiated and let $\left(p^{*}, q^{*}, x^{*}, y^{*}\right)$ be a sophisticated $S C$ equilibrium of $\mathcal{E}$. Then for every $s, s^{\prime} \in S$ and every $t, \tau \in T$ with $\tau>t$ and $s^{\prime}(t)=s(t)$ there is a $\mu_{s^{\prime}(\tau) \mid s(t)} \in \mathbb{R}$ such that

$$
q_{s^{\prime}(\tau) \mid s(t)}^{*}=\mu_{s^{\prime}(\tau) \mid s(t)} p_{s^{\prime}(\tau) \mid s(t)}^{*} .
$$


An $\mathrm{AD}$ equilibrium $\left(p^{*}, x^{*}\right)$ is said to be allocationally equivalent to a naïve (sophisticated) SC equilibrium if there are $p \in P, q \in \bar{Q}$ and $y \in \bar{Y}$ such that $\left(p, q, x^{*}, y\right)$ is a naïve (sophisticated) SC equilibrium. Similarly, a naïve (sophisticated) SC equilibrium $\left(p^{*}, q^{*}, x^{*}, y^{*}\right)$ is said to be allocationally equivalent to an $\mathrm{AD}$ equilibrium if there is $p \in P$ such that $\left(p, x^{*}\right)$ is an $\mathrm{AD}$ equilibrium.

When preferences are time-consistent one might expect that there is no difference between naïve and sophisticated behavior. However, this is not the case. We show by means of an example that naïve SC equilibria are not necessarily allocationally equivalent to AD equilibria. However, sophisticated SC equilibria are allocationally equivalent to AD equilibria and the other way around. The difference arises because in an $\mathrm{AD}$ equilibrium price expectations are correct, whereas in a naïve SC equilibrium price expectations are only rational. The key insight used in the example is that even in the time-consistent case, rational price expectations need not be correct. Finally, every AD equilibrium is allocationally equivalent to some SC equilibrium in both the naïve and the sophisticated case. These results will be derived next.

The following example with time-consistent preferences displays naïve SC equilibria that are not allocationally equivalent to any $\mathrm{AD}$ equilibrium. It is borrowed from Drèze and Herings (2003). Hellwig (1983) considered a similar example for the case with incomplete markets.

Example 6.7 Consider a two-period economy without uncertainty. There are two agents and two goods in every period. Endowments are $e_{\cdot \mid 1}^{h}=(2,2,2,2)$ for $h=1,2$. Preferences are time-consistent and represented by

$$
u_{1}^{h}\left(x_{\cdot \mid 1}^{h}\right)=v^{h}\left(x_{1,1 \mid 1}^{h}, x_{1,2 \mid 1}^{h}\right)^{\frac{1}{2}} \cdot v^{h}\left(x_{2,1 \mid 1}^{h}, x_{2,2 \mid 1}^{h}\right)^{\frac{1}{2}}
$$

where

$$
v^{1}\left(x_{\cdot, 1 \mid 1}^{1}, x_{\cdot, 2 \mid 1}^{1}\right)= \begin{cases}{\left[\min \left(x_{\cdot, 1 \mid 1}^{1}, x_{\cdot, 2 \mid 1}^{1}\right)\right],} & \min \left(x_{\cdot, 1 \mid 1}^{1}, x_{\cdot, 2 \mid 1}^{1}\right) \leq 1, \\ {\left[\left(x_{\cdot, 1 \mid 1}^{1}-1\right)^{\frac{1}{2}}\left(x_{\cdot, 2 \mid 1}^{1}-1\right)^{\frac{1}{2}}+1\right],} & \min \left(x_{\cdot, 1 \mid 1}^{1}, x_{\cdot, 2 \mid 1}^{1}\right) \geq 1,\end{cases}
$$

and

$$
v^{2}\left(x_{\cdot, 1 \mid 1}^{2}, x_{\cdot, 2 \mid 1}^{2}\right)= \begin{cases}{\left[\min \left(x_{\cdot, 1 \mid 1}^{2}, x_{\cdot, 2 \mid 1}^{2}\right)\right],} & \min \left(x_{\cdot, 1 \mid 1}^{2}, x_{\cdot, 2 \mid 1}^{2}\right) \leq 3, \\ {\left[\left(x_{\cdot, 1 \mid 1}^{2}-3\right)^{\frac{1}{2}}\left(x_{\cdot, 2 \mid 1}^{2}-3\right)^{\frac{1}{2}}+3\right],} & \min \left(x_{\cdot, 1 \mid 1}^{2}, x_{\cdot, 2 \mid 1}^{2}\right) \geq 3 .\end{cases}
$$


Now consider sequentially complete markets. Let $p_{\cdot \mid 1}^{*}=(1 / 2,1 / 2,1 / 2,1 / 2)$, $q_{2 \mid 1}^{*}=(1 / 2,1 / 2), p_{\cdot \mid 2}^{*}=(3 / 4,1 / 4)$. Consider the following allocation

$$
\begin{array}{ll}
x_{\cdot \mid 1}^{* 1}=(2,2,2,2) & x_{\cdot \mid 1}^{* 2}=(2,2,2,2) \\
x_{\cdot \mid 2}^{* 1}=(1,1) & x_{\cdot \mid 2}^{* 2}=(3,3)
\end{array}
$$

achieved by the asset allocations

$$
\begin{aligned}
& y_{2 \mid 1}^{* 1}=(0,4), \\
& y_{2 \mid 1}^{* 2}=(4,0) .
\end{aligned}
$$

It can be verified that $\left(p^{*}, q^{*}, x^{*}, y^{*}\right)$ is a naïve SC equilibrium. In particular it holds that the choice of $y_{2 \mid 1}^{* 1}=(0,4)$ and $y_{2 \mid 1}^{* 2}=(4,0)$ is optimal given prices and price expectations $p_{. \mid 1}^{*}=(1 / 2,1 / 2,1 / 2,1 / 2)$ and it holds that for the subeconomy starting in period 2 with initial endowments $y_{2 \mid 1}^{* 1}=(0,4)$ and $y_{2 \mid 1}^{* 2}=(4,0)$, prices $p_{\cdot \mid 2}^{*}=(3 / 4,1 / 4)$ constitute a competitive equilibrium. The prices for contingent commodities in period 1 are not proportional to actual prices in period 2. Thus, in period 1 households have rational expectations about prices in period 2 , but in period 2 they are confronted with different competitive equilibrium prices. Since $x_{\cdot \mid 1}^{1}=(2,2,1,1)$ is not individually rational for household 1 from the perspective of period 1 , there can be no $p$ such that $(p,(2,2,1,1),(2,2,3,3))$ is an $\mathrm{AD}$ equilibrium.

When preferences are time-consistent, every $\mathrm{AD}$ equilibrium is allocationally equivalent to some naïve and to some sophisticated SC equilibrium. This is shown in the following theorems. Crucial in the derivation of the first result is that the set of correct price expectations is a subset of the set of rational price expectations.

Theorem 6.8 Let preferences be time-consistent and let the economy $\mathcal{E}$ be locally non-satiated. Let $\left(p^{*}, x^{*}\right)$ be an $A D$ equilibrium of $\mathcal{E}$. Then there is $\left(q^{*}, y^{*}\right) \in \bar{Q} \times \bar{Y}$ such that $\left(p^{*}, q^{*}, x^{*}, y^{*}\right)$ is a naïve $S C$ equilibrium of $\mathcal{E}$.

Theorem 6.9 Let preferences be time-consistent and let the economy $\mathcal{E}$ be locally non-satiated. Let $\left(p^{*}, x^{*}\right)$ be an $A D$ equilibrium of $\mathcal{E}$. Then there is $\left(q^{*}, y^{*}\right) \in \bar{Q} \times \bar{Y}$ such that $\left(p^{*}, q^{*}, x^{*}, y^{*}\right)$ is a sophisticated $S C$ equilibrium of $\mathcal{E}$.

Every sophisticated SC equilibrium is allocationally equivalent to some AD equilibrium if preferences are time-consistent, as is shown next. The fact that sophisticated households have correct price expectations is important for deriving this result. Notice that we need to make some additional assumptions for this 
direction, because we need optimal consumption plans to exist in the opportunity sets. The preferences of household $h$ are said to be acyclic if for every state $s(t)$ there is no finite set $\left\{x_{\cdot \mid s(t)}^{h, 1}, \ldots, x_{\cdot \mid s(t)}^{h, n}\right\} \subset X_{\cdot \mid s(t)}^{h}$ such that $x_{\cdot \mid s(t)}^{h, i} \succ^{h, s(t)} x_{\cdot \mid s(t)}^{h, i+1}$ for $i=1, \ldots, n-1$ and such that $x_{\cdot \mid s(t)}^{h, n} \succ^{h, s(t)} x_{\cdot \mid s(t)}^{h, 1}$. The preferences of household $h$ are said to have open lower sections if for every state $s(t)$, for every $x_{\cdot \mid s(t)}^{h} \in X_{\cdot \mid s(t)}^{h}$, the set $\left\{\widehat{x}_{\cdot \mid s(t)}^{h} \in X_{\cdot \mid s(t)}^{h} \mid x_{\cdot \mid s(t)}^{h} \succ^{h, s(t)} \widehat{x}_{\cdot \mid s(t)}^{h}\right\}$ is open in $X_{\cdot \mid s(t)}^{h}$.

Theorem 6.10 Let preferences be time-consistent, locally non-satiated, acyclic, and have open lower sections, and let consumption sets be closed and bounded from below. Let $\left(p^{*}, q^{*}, x^{*}, y^{*}\right)$ be a sophisticated $S C$ equilibrium of $\mathcal{E}$ with $p^{*} \gg 0$. Then there is a $p$ such that $\left(p, x^{*}\right)$ is an $A D$ equilibrium of $\mathcal{E}$.

In general, when preferences might be time-inconsistent, there is no relation between AD equilibria and naïve or sophisticated SC equilibria. When preferences are time-inconsistent conflicts arise between current and future preferences. A naïve household will typically deviate from planned consumption, since he does not anticipate a change in his future preferences. Sophisticated households do anticipate future changes, and are therefore constrained by their future behavior. On the contrary, the AD market setting ensures perfect commitment in period 1, which is optimal from the perspective of the preferences of households in period 1. Constraints coming from the behavior of future selves are thereby irrelevant.

\subsection{Complete Markets}

In this section there are complete markets for all possible contingent commodities at every state of every period. Again, $p_{\cdot \mid s(t)}$ denotes the expected (at state $s(t)$ ) prices on the spot markets. In state $s(t)$, the expected state $s^{\prime}(\tau)$ prices for contingent commodities that are delivered in state $s^{\prime \prime}(\tilde{\tau})$ are given by $q_{\left(s^{\prime \prime}(\tilde{\tau}) \mid s^{\prime}(\tau)\right) \mid s(t)}$, where $\tilde{\tau}>\tau \geq t, s^{\prime \prime}(\tau)=s^{\prime}(\tau)$, and $s^{\prime}(t)=s(t)$. The expectations in state $s(t)$ of prices for all 'future' contingent commodities that can possibly be traded from state $s^{\prime}(\tau)$ on, are denoted by $q_{\left(\cdot \mid s^{\prime}(\tau), T\right) \mid s(t)}$. We define $Q \cdot \mid s(t)=\mathbb{R}^{\sum_{\tilde{\tau}=t}^{T-1} \mathcal{L}_{\tilde{\tau}}}$. We let $y_{\left(s^{\prime}(\tilde{\tau}) \mid s^{\prime}(\tau)\right) \mid s(t)}^{h}$ denote bundles of contingent commodities that are expected at $s(t)$ to be bought in $s^{\prime}(\tau)$ and that are delivered in $s^{\prime}(\tilde{\tau})$. For notational purposes, we define $y_{(\cdot \mid s(0)) \mid s(0)}^{h}=e_{\cdot \mid 1}^{h}$. We also define $Y_{\cdot \mid s(t)}^{h}=\mathbb{R}^{\sum_{\tilde{\tau}=t}^{T-1} \mathcal{L}_{\tilde{\tau}}}$. Finally,

$$
\begin{array}{ll}
X=\prod_{h \in H} \prod_{s(t)} X_{\cdot \mid s(t)}^{h}, \quad P=\prod_{s(t)} P_{\cdot \mid s(t)}, \\
Y=\prod_{h \in H} \prod_{s(t)} Y_{\cdot \mid s(t)}^{h}, \quad Q=\prod_{s(t)} Q_{\cdot \mid s(t)} .
\end{array}
$$




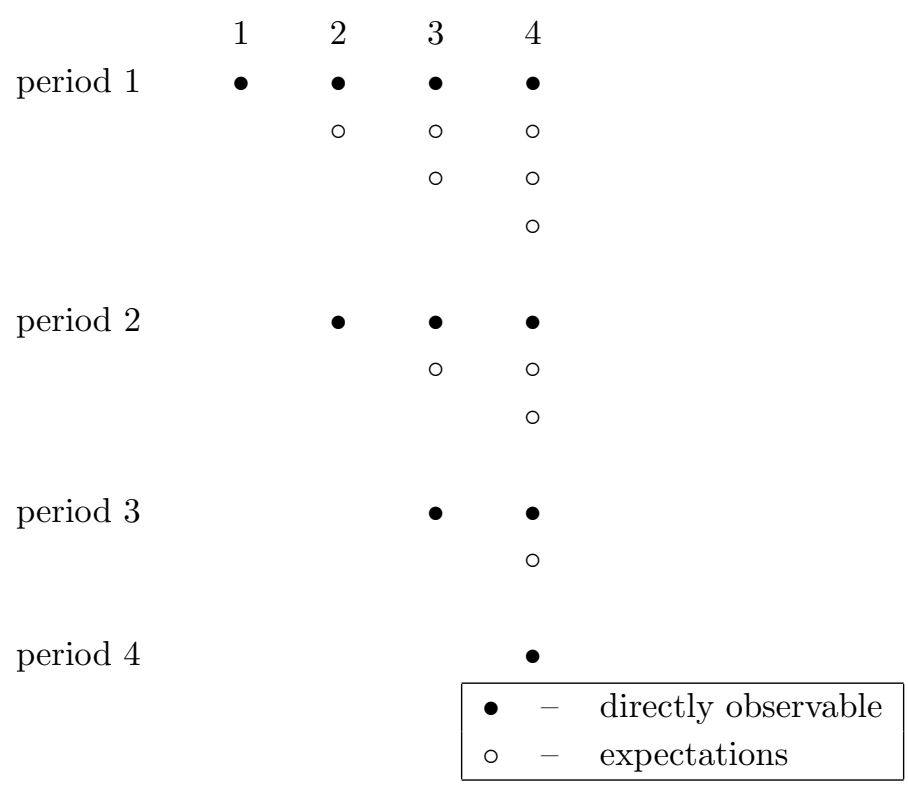

Figure 6.8: Structure of complete markets

Figure 6.8 illustrates the structure of complete markets and is analogous to Figure 6.7.

\subsubsection{Naïve Behavior}

In every state households sell their endowments which are determined by previous purchases of contingent commodities. With the revenues of these sales they buy goods on spot markets and contingent commodities on asset markets. The contingent commodities bought determine the endowments at future states. In order to make a choice, a household needs to have expectations about prices of goods and contingent commodities in the future so as to know what to buy. As before, naïve households have rational price expectations. Thus, they all have the same point expectations about prices on spot and forward-commodity markets and prices are expected to be market clearing. We will show later that, in equilibrium, households cannot expect prices to change a lot, since that would make them believe that there are arbitrage opportunities, so that demand of some commodities would be infinite. In every period households form new rational price expectations. The new expectations need not be equal to the expectations from the period before. 
This again is due to the changing preferences. This does not contradict with the fact that previous expectations were rational, since naïve households always have the incorrect belief that their preferences will not change. Again, rational price expectations are correct price expectations given this belief. The opportunity set of household $h$ in state $s(t)$ is given by

$$
\begin{gathered}
{ }^{n} \gamma_{s(t)}^{h}\left(p_{\cdot \mid s(t)}, q_{\cdot \mid s(t)}, y_{(\cdot \mid s(t-1)) \mid s(t-1)}^{h}\right)=\left\{\left(x_{\cdot \mid s(t)}^{h}, y_{\cdot \mid s(t)}^{h}\right) \in X_{\cdot \mid s(t)}^{h} \times Y_{\cdot \mid s(t)}^{h} \mid\right. \\
p_{s^{\prime}(\tau) \mid s(t)} x_{s^{\prime}(\tau) \mid s(t)}^{h}+q_{\left(\cdot \mid s^{\prime}(\tau)\right) \mid s(t)} y_{\left(\cdot \mid s^{\prime}(\tau)\right) \mid s(t)}^{h} \leq \\
p_{s^{\prime}(\tau) \mid s(t)} y_{\left(s^{\prime}(\tau) \mid s^{\prime}(\tau-1)\right) \mid s(t)}^{h}+q_{\left(\cdot \mid s^{\prime}(\tau)\right) \mid s(t)} y_{\left(s^{\prime+}(\tau), T \mid s^{\prime}(\tau-1)\right) \mid s(t)}^{h} \\
\text { for every } \left.\tau \geq t, \text { and every } s^{\prime}(\tau) \text { with } s^{\prime}(t)=s(t)\right\},
\end{gathered}
$$

where, by definition, $y_{(\cdot \mid s(0)) \mid s(0)}^{h}=e_{\cdot \mid 1}^{h}$ and $y_{(\cdot \mid s(t-1)) \mid s(t)}^{h}=y_{(\cdot \mid s(t-1)) \mid s(t-1)}^{h}$. The demand set is then given by

$$
\begin{aligned}
& { }^{n} \delta_{s(t)}^{h}\left(p_{\cdot \mid s(t)}, q_{\cdot \mid s(t)}, y_{(\cdot \mid s(t-1)) \mid s(t-1)}^{h}\right)= \\
& \left\{\left(\bar{x}_{\cdot \mid s(t)}^{h}, \bar{y}_{\cdot \mid s(t)}^{h}\right) \in{ }^{n} \gamma_{s(t)}^{h}\left(p_{\cdot \mid s(t)}, q_{\cdot \mid s(t)}, y_{(\cdot \mid s(t-1)) \mid s(t-1)}^{h}\right) \mid\right. \\
& \nexists\left(x_{\cdot \mid s(t)}^{h}, y_{\cdot \mid s(t)}^{h}\right) \in{ }^{n} \gamma_{s(t)}^{h}\left(p_{\cdot \mid s(t)}, q \cdot \mid s(t), y_{(\cdot \mid s(t-1)) \mid s(t-1)}^{h}\right) \\
& \left.\quad \text { with } x_{\cdot \mid s(t)}^{h} \succ^{h, s(t)} \bar{x}_{\cdot \mid s(t)}^{h}\right\} .
\end{aligned}
$$

In equilibrium all markets should clear and should be expected to clear.

\section{Definition 6.11 Naïve Complete Equilibrium}

A pair $\left(p^{*}, q^{*}, x^{*}, y^{*}\right) \in P \times Q \times X \times Y$ is a Naïve Complete equilibrium of the economy $\mathcal{E}$ if

(a) $\left(x_{\cdot \mid s(t)}^{* h}, y_{\cdot \mid s(t)}^{* h}\right) \in{ }^{n} \delta_{s(t)}^{h}\left(p_{\cdot \mid s(t)}^{*}, q_{\cdot \mid s(t)}^{*}, y_{(\cdot \mid s(t-1)) \mid s(t-1)}^{* h}\right)$ for all $h \in H, s \in S, t \in T$,

(b) $\sum_{h \in H} x_{\cdot \mid s(t)}^{* h}=\sum_{h \in H} e_{\cdot \mid s(t)}^{h}$ for all $s \in S, t \in T$, and

(c) $\sum_{h \in H} y_{(\cdot \mid s(\tau)) \mid s(t)}^{* h}=\sum_{h \in H} e_{\tau+1, T \mid s(\tau)}^{h}$ for all $s \in S, t, \tau \in T$ with $t \leq \tau$.

\subsubsection{Sophisticated Behavior}

In period $T$ the opportunity set of a sophisticated household $h$ in state $s$ is the same as the opportunity set for its naïve counterpart, i.e.

$$
{ }^{s} \gamma_{s(T)}^{h}\left(p_{\cdot \mid s(T)}, y_{\cdot \mid s(T-1)}^{h}\right)={ }^{n} \gamma_{s(T)}^{h}\left(p_{\cdot \mid s(T)}, y_{\cdot \mid s(T-1)}^{h}\right) .
$$

The opportunity set of a sophisticated household $h$ in state $s(t)$, where $t<T$, is given by 


$$
{ }^{s} \gamma_{s(t)}^{h}\left(p_{\cdot \mid s(t)}, q_{\cdot \mid s(t)}, y_{(\cdot \mid s(t-1)) \mid s(t-1)}^{h}\right)=\left\{\left(x_{\cdot \mid s(t)}^{h}, y_{\cdot \mid s(t)}^{h}\right) \in X_{\cdot \mid s(t)}^{h} \times Y_{\cdot \mid s(t)}^{h} \mid\right.
$$

(i) $p_{s^{\prime}(\tau) \mid s(t)} x_{s^{\prime}(\tau) \mid s(t)}^{h}+q_{\left(\cdot \mid s^{\prime}(\tau)\right) \mid s(t)} y_{\left(\cdot \mid s^{\prime}(\tau)\right) \mid s(t)}^{h} \leq$

$$
p_{s^{\prime}(\tau) \mid s(t)} y_{\left(s^{\prime}(\tau) \mid s^{\prime}(\tau-1)\right) \mid s(t)}^{h}+q_{\left(\cdot \mid s^{\prime}(\tau)\right) \mid s(t)} y_{\left(s^{\prime+}(\tau), T \mid s^{\prime}(\tau-1)\right) \mid s(t)}^{h}
$$

for every $\tau \geq t$, and every $s^{\prime}(\tau)$ with $s^{\prime}(t)=s(t)$,

and

$$
\begin{aligned}
(i i)\left(x_{s(t+1), T \mid s(t)}^{h}, y_{(\cdot \mid s(t+1), T) \mid s(t)}^{h}\right) \in & \\
& { }^{s} \delta_{s(t+1)}^{h}\left(p_{s(t+1), T \mid s(t)}, q_{(\cdot \mid s(t+1), T) \mid s(t)}, y_{(\cdot \mid s(t)) \mid s(t)}^{h}\right) \\
& \text { for every } \left.s(t+1) \in s^{+}(t)\right\} .
\end{aligned}
$$

The demand set of a sophisticated household is as follows.

$$
\begin{aligned}
& { }^{s} \delta_{s(t)}^{h}\left(p_{\cdot \mid s(t)}, q \cdot \mid s(t), y_{(\cdot \mid s(t-1)) \mid s(t-1)}^{h}\right)= \\
& \left\{\left(\bar{x}_{\cdot \mid s(t)}^{h}, \bar{y}_{\cdot \mid s(t)}^{h}\right) \in{ }^{s} \gamma_{s(t)}^{h}\left(p_{\cdot \mid s(t)}, q_{\cdot \mid s(t)}, y_{(\cdot \mid s(t-1)) \mid s(t-1)}^{h}\right) \mid\right. \\
& \quad \nexists\left(x_{\cdot \mid s(t)}^{h}, y_{\cdot \mid s(t)}^{h}\right) \in{ }^{s} \gamma_{s(t)}^{h}\left(p_{\cdot \mid s(t)}, q \cdot \mid s(t), y_{(\cdot \mid s(t-1)) \mid s(t-1)}^{h}\right) \\
& \left.\quad \text { with } x_{\cdot \mid s(t)}^{h} \succ^{h, s(t)} \bar{x}_{\cdot \mid s(t)}^{h}\right\} .
\end{aligned}
$$

Finally, we define a sophisticated complete equilibrium as follows. Note that the difference with a naïve complete equilibrium is that sophisticated households have correct price expectations and correct expectations about future purchases.

\section{Definition 6.12 Sophisticated Complete Equilibrium}

A pair $\left(p^{*}, q^{*}, x^{*}, y^{*}\right) \in P \times Q \times X \times Y$ is a Sophisticated Complete equilibrium of the economy $\mathcal{E}$ if

(a) $\left(x_{\cdot \mid s(t)}^{* h}, y_{\cdot \mid s(t)}^{* h}\right) \in{ }^{s} \delta_{s(t)}^{h}\left(p_{\cdot \mid s(t)}^{*}, q_{\cdot \mid s(t)}^{*}, y_{(\cdot \mid s(t-1)) \mid s(t-1)}^{* h}\right)$ for all $h \in H, s \in S, t \in T$,

(b) $\sum_{h \in H} x_{\cdot \mid s(t)}^{* h}=\sum_{h \in H} e_{\cdot \mid s(t)}^{h}$ for all $s \in S, t \in T$,

(c) $\sum_{h \in H} y_{(\cdot \mid s(\tau)) \mid s(t)}^{* h}=\sum_{h \in H} e_{\tau+1, T \mid s(\tau)}^{h}$ for all $s \in S, t, \tau \in T$ with $t \leq \tau$,

(d) $p_{s(\tau), T \mid s(t)}^{*}=p_{\cdot \mid s(\tau)}^{*}$ for all $s \in S, t, \tau \in T$ with $t \leq \tau$,

(e) $q_{(\cdot \mid s(\tau), T) \mid s(t)}^{*}=q_{\cdot \mid s(\tau)}^{*}$ for all $s \in S, t, \tau \in T$ with $t \leq \tau$,

(f) $x_{s(\tau), T \mid s(t)}^{* h}=x_{\cdot \mid s(\tau)}^{* h}$ for all $h \in H, s \in S, t, \tau \in T$ with $t \leq \tau$, and

(g) $y_{(\cdot \mid s(\tau), T) \mid s(t)}^{* h}=y_{\cdot \mid s(\tau)}^{* h}$ for all $h \in H, s \in S, t, \tau \in T$ with $t \leq \tau$. 


\subsubsection{Properties of Complete Equilibria}

The essential difference between complete (C) and sequentially complete (SC) markets is that in complete markets there is a richer set of assets available. As a consequence, in a 3-period example for instance, under $\mathrm{C}$ it is possible in period 1 to make binding commitments regarding the delivery and purchases of commodities in periods 2 and 3 . When price expectations regarding periods 2 and 3 do not change, this is inconsequential. All that matters is available wealth in each period, and not the composition of wealth, i.e. the distribution of wealth over the various assets. When price expectations do change, different compositions of wealth typically do have real consequences. Under SC there is less choice for the composition of wealth. In this section we will therefore address the question of how complete and sequentially complete equilibria relate to one another.

An $\mathrm{AD}$ equilibrium $\left(p^{*}, q^{*}\right)$ is said to be allocationally equivalent to a naïve (sophisticated) complete equilibrium if there are $p \in P, q \in Q$ and $y \in Y$ such that $\left(p, q, x^{*}, y\right)$ is a naïve (sophisticated) complete equilibrium. Similarly, a naïve (sophisticated) complete equilibrium $\left(p^{*}, q^{*}, x^{*}, y^{*}\right)$ is said to be allocationally equivalent to an $\mathrm{AD}$ equilibrium if there is a $p \in P$ such that $\left(p, x^{*}\right)$ is an $\mathrm{AD}$ equilibrium. A naïve (sophisticated) complete equilibrium $\left(p^{*}, q^{*}, x^{*}, y^{*}\right)$ is said to be allocationally equivalent to a naïve (sophisticated) SC equilibrium if there are $p \in P$, $q \in \bar{Q}$ and $y \in \bar{Y}$ such that $\left(p, q, x^{*}, y\right)$ is a naïve (sophisticated) SC equilibrium. A naïve (sophisticated) SC equilibrium $\left(p^{*}, q^{*}, x^{*}, y^{*}\right)$ is said to be allocationally equivalent to a naïve (sophisticated) complete equilibrium if there are $p \in P, q \in Q$ and $y \in Y$ such that $\left(p, q, x^{*}, y\right)$ is a naïve (sophisticated) complete equilibrium.

In complete equilibria, relative prices are expected to remain unchanged. This is proven in the following theorem.

Theorem 6.13 Let the economy $\mathcal{E}$ be locally non-satiated and let $\left(p^{*}, q^{*}, x^{*}, y^{*}\right)$ be a naive (sophisticated) complete equilibrium of $\mathcal{E}$. Then for every $s, s^{\prime}$ and every $t, \tau$ with $t<\tau$ there must be $\mu_{s^{\prime}(\tau) \mid s(t)} \in \mathbb{R}$ with

$$
q_{\left(s^{\prime}(\tau), T \mid s^{\prime}(\tau-1)\right) \mid s(t)}^{*}=\mu_{s^{\prime}(\tau) \mid s(t)}\left(p_{s^{\prime}(\tau) \mid s(t)}^{*}, q_{\left(\cdot \mid s^{\prime}(\tau)\right) \mid s(t)}^{*}\right) .
$$

In general, when preferences are allowed to be time-inconsistent, there is no link between naïve (sophisticated) complete equilibria and $\mathrm{AD}$ equilibria. In $\mathrm{AD}$ equilibria, because of the commitment power provided by the AD market structure, future selves of households have no influence. In naïve complete equilibria, future selves will typically deviate from the plans of current selves. In sophisticated complete equilibria, current selves are constrained by the optimizing behavior of future selves. 
When preferences are time-consistent, every $\mathrm{AD}$ equilibrium is allocationally equivalent to some naïve (sophisticated) complete equilibrium. This is shown in the following two theorems.

Theorem 6.14 Let preferences in the economy $\mathcal{E}$ be time-consistent and let $\left(p^{*}, x^{*}\right)$ be an $A D$ equilibrium of $\mathcal{E}$. Then $\left(p^{*}, q^{*}, x^{*}, y^{*}\right)$ is a naïve complete equilibrium of $\mathcal{E}$, where $y_{\left(s^{\prime}(\tilde{\tau}) \mid s^{\prime}(\tau)\right) \mid s(t)}^{* h}=x_{s^{\prime}(\tilde{\tau}) \mid s(t)}^{* h}$ for every $h, s^{\prime}, s, \tilde{\tau}>\tau \geq t$ and $q_{\left(s^{\prime}(\tilde{\tau}) \mid s^{\prime}(\tau)\right) \mid s(t)}^{*}$ $=p_{s^{\prime}(\tilde{\tau}) \mid s(t)}^{*}$ for every $s^{\prime}, s, \tilde{\tau}>\tau \geq t$.

Theorem 6.15 Let preferences in the economy $\mathcal{E}$ be time-consistent and let $\left(p^{*}, x^{*}\right)$ be an $A D$ equilibrium of $\mathcal{E}$. Then $\left(p^{*}, q^{*}, x^{*}, y^{*}\right)$ is a sophisticated complete equilibrium of $\mathcal{E}$, where $y_{\left(s^{\prime}(\tilde{\tau}) \mid s^{\prime}(\tau)\right) \mid s(t)}^{* h}=x_{s^{\prime}(\tilde{\tau}) \mid s(t)}^{* h}$ for every $h, s^{\prime}, s, \tilde{\tau}>\tau \geq t$ and $q_{\left(s^{\prime}(\tilde{\tau}) \mid s^{\prime}(\tau)\right) \mid s(t)}^{*}=p_{s^{\prime}(\tilde{\tau}) \mid s(t)}^{*}$ for every $s^{\prime}, s, \tilde{\tau}>\tau \geq t$.

The following example shows that even when preferences are time-consistent, not every naïve complete equilibrium is allocationally equivalent to an AD equilibrium. As before, this result arises because naïve households form only rational price expectations.

Example 6.16 Consider Example 6.7. For two-period economies, the sequentially complete market structure is identical to the complete market structure. Thus, $\left(p^{*}, q^{*}, x^{*}, y^{*}\right)$ is also a naïve complete equilibrium. Again, there is no $p$ such that $(p,(2,2,1,1),(2,2,3,3))$ is an $\mathrm{AD}$ equilibrium.

Every sophisticated complete equilibrium, however, is allocationally equivalent to some $\mathrm{AD}$ equilibrium when preferences are time-consistent. Again, we need to make some additional assumptions.

Theorem 6.17 Let preferences be time-consistent, locally non-satiated, acyclic, and have open lower sections, and let consumption sets be closed and bounded from below. Let $\left(p^{*}, q^{*}, x^{*}, y^{*}\right)$ be a sophisticated complete equilibrium of $\mathcal{E}$ with $p^{*} \gg 0$. Then $\left(p, x^{*}\right)$ is an $A D$ equilibrium of $\mathcal{E}$, where $p_{1 \mid 1}=p_{1 \mid 1}^{*}$ and $p_{2, T \mid 1}=q_{2, T|1| 1}^{*}$.

So far we have studied the relation between $\mathrm{AD}$ equilibria on the one hand and $\mathrm{SC}$ and complete equilibria on the other hand. Now we will analyze the relation between the latter two. The next theorem shows that every naïve SC equilibrium is allocationally equivalent to some naïve complete equilibrium. Notice that there is no need to assume time-consistency to obtain this result.

Theorem 6.18 Let the economy $\mathcal{E}$ be locally non-satiated and let $\left(p^{*}, q^{*}, x^{*}, y^{*}\right)$ be a naïve $S C$ equilibrium of $\mathcal{E}$. Then there is $(p, q, y) \in P \times Q \times Y$ such that $\left(p, q, x^{*}, y\right)$ is a naïve complete equilibrium of $\mathcal{E}$. 
Surprisingly, there are naïve complete equilibria that are not allocationally equivalent to any naïve SC equilibrium, even when preferences are time-consistent. This is shown in the following example. The key insight is that the richer set of assets available under complete markets may lead to initial endowments in future periods that are not feasible under sequentially complete markets and that admit continuation equilibria that are not feasible in the sequentially complete markets setting.

Example 6.19 Consider an economy with three periods, no uncertainty, one good per period, and two households. Assume that each household initially owns one unit of the good in period 1 and two units of the good in period 2 and period 3 , i.e. $e_{\cdot \mid 1}^{1}=e_{\cdot \mid 1}^{2}=(1,2,2)$.

Assume that both households have time-consistent preferences represented by the utility function

$$
u^{h}\left(x_{1 \mid 1}^{h}, x_{2 \mid 1}^{h}, x_{3 \mid 1}^{h}\right)=\sqrt{x_{1 \mid 1}^{h}}+\sqrt{x_{2 \mid 1}^{h}} \sqrt{x_{3 \mid 1}^{h}}+\sqrt{x_{3 \mid 1}^{h}} .
$$

Consider the complete market structure. Suppose that prices and price expectations in period 1 are given by $p_{\cdot \mid 1}^{*}=(1,1,1+\sqrt{2} / 2), q_{(\cdot \mid 1) \mid 1}^{*}=(1,1+\sqrt{2} / 2)$, and $q_{(\cdot \mid 2) \mid 1}^{*}=1+\sqrt{2} / 2$. Consider the case where price expectations are correct, so $p_{\cdot \mid 2}^{*}=(1,1+\sqrt{2} / 2), q_{(\cdot \mid 2) \mid 2}^{*}=1+\sqrt{2} / 2$, and $p_{\cdot \mid 3}^{*}=1+\sqrt{2} / 2$. It can easily be shown that with these prices both households expect to consume and actually will consume their initial endowments. Thus, these prices together with the consumption of the endowments and no trade in contingent commodities constitute a naïve complete equilibrium. It is easily verified that the same allocation can also be sustained by a naïve sequentially complete equilibrium.

In the complete market structure households have the option to trade contingent commodities in period 1 while still consuming their endowments in period 1 . Let prices and price expectations in period 1 be as before, $\bar{p}_{\cdot \mid 1}=(1,1,1+\sqrt{2} / 2)$, $\bar{q}_{(\cdot \mid 1) \mid 1}=(1,1+\sqrt{2} / 2)$, and $\bar{q}_{(\cdot \mid 2) \mid 1}=1+\sqrt{2} / 2$. Suppose that in period 1 households trade contingent commodities for period 2 and period 3 in such a way that

$$
\begin{aligned}
\bar{x}_{1 \mid 1}^{1} & =1 \\
\bar{y}_{(2 \mid 1) \mid 1}^{1} & =4+\sqrt{2}-\frac{2+\sqrt{2}}{2} \frac{8+8 \sqrt{2}}{4 \sqrt{2}-2 \sqrt{3}-2}, \\
\bar{y}_{(3 \mid 1) \mid 1}^{1} & =\frac{8+8 \sqrt{2}}{4 \sqrt{2}-2 \sqrt{3}-2} .
\end{aligned}
$$

Market clearing requires that $\bar{y}_{(2 \mid 1) \mid 1}^{2}=4-\bar{y}_{(2 \mid 1) \mid 1}^{1}$ and $\bar{y}_{(3 \mid 1) \mid 1}^{2}=4-\bar{y}_{(3 \mid 1) \mid 1}^{1}$. Notice that for $h=1,2, \bar{q}_{(2 \mid 1) \mid 1} y_{(2 \mid 1) \mid 1}^{h}+\bar{q}_{(3 \mid 1) \mid 1} y_{(3 \mid 1) \mid 1}^{h}=\bar{q}_{(2 \mid 1) \mid 1} e_{2 \mid 1}^{h}+\bar{q}_{(3 \mid 1) \mid 1} e_{3 \mid 1}^{h}$. 
Households plan to retrade in period 2 , for $h=1,2$,

$$
\bar{y}_{(3 \mid 2) \mid 1}^{h}=2,
$$

so $\bar{x}_{\cdot \mid 1}^{h}=(1,2,2)$.

Assume realized and expected prices in period 2 to be given by

$$
\begin{aligned}
\bar{p}_{\cdot \mid 2} & =\left(1, \frac{5+\sqrt{3}}{4}\right), \\
\bar{q}_{(3 \mid 2) \mid 2} & =\frac{5+\sqrt{3}}{4} .
\end{aligned}
$$

Then the optimal consumption for household 1 is $\bar{x}_{\cdot \mid 2}^{1}=(1,8 /(5+\sqrt{3}))$ and for household $2, \bar{x}_{\cdot \mid 2}^{2}=(3,4-8 /(5+\sqrt{3}))$. In period 3 it will follow that $\bar{x}_{3 \mid 3}^{1}=$ $\bar{y}_{(3 \mid 2) \mid 2}^{1}=8 /(5+\sqrt{3})$, and $\bar{x}_{3 \mid 3}^{2}=\bar{y}_{(3 \mid 2) \mid 2}^{2}=4-8 /(5+\sqrt{3})$. It can be verified that $(\bar{p}, \bar{q}, \bar{x}, \bar{y})$ is a naïve complete equilibrium.

In sequentially complete markets consuming the endowment in the first period implies no trade of contingent commodities. Therefore the allocation $\bar{x}$ cannot be achieved in a naïve sequentially complete equilibrium.

We have already mentioned that there is no relation between $\mathrm{AD}$ equilibria on the one hand and naïve (sophisticated) SC or naïve (sophisticated) complete equilibria on the other hand when preferences are not time-consistent. Theorem 6.18 shows that every naïve SC equilibrium is allocationally equivalent to some complete equilibrium and Example 6.19 shows that the converse is not true. When we consider sophisticated behavior, however, sophisticated SC equilibria are allocationally equivalent to sophisticated complete equilibria and vice versa. This is shown in the following theorem.

Theorem 6.20 Let the economy $\mathcal{E}$ be locally non-satiated. Every sophisticated $S C$ equilibrium of $\mathcal{E}$ is allocationally equivalent to some sophisticated complete equilibrium of $\mathcal{E}$ and vice versa.

\subsection{Conclusion}

Arrow (1953) showed that every Pareto optimal allocation in an Arrow-Debreu economy can be achieved both by Arrow-Debreu markets and a particular sequentially complete market setting where first securities are traded and then spot markets are opened. Debreu (1959) claims that, when all contingent commodities can be traded in the first period and preferences of households do not change over time, there is no need for markets to reopen in later periods. 
In this chapter we allow preferences to be time-inconsistent and we analyze three market structures. In this richer environment, we reconsider the results of Arrow (1953) and Debreu (1959).

We first examine the Arrow-Debreu setting where all contingent commodities can be traded in the first period and where markets are not reopened in later periods. Next, we consider sequentially complete markets where goods on the spot markets and all one-period ahead contingent commodities can be traded in every state. Finally, we consider complete markets where all contingent commodities can be traded in every state.

Following Pollak (1968) we make a distinction between naïve and sophisticated behavior. Naïve households do not realize that their preferences change over time, whereas sophisticated ones do. The latter will only make plans that they expect to carry out in the future.

For sophisticated households, we show that sequentially complete markets are allocationally equivalent to complete markets. For naïve households, every naïve sequentially complete equilibrium is allocationally equivalent to some naïve complete equilibrium, but the converse does not hold, even if we assume that preferences are time-consistent.

When preferences are time-consistent and households are naïve, every equilibrium in the Arrow-Debreu setting is allocationally equivalent to some naïve sequentially complete equilibrium and to some naïve complete equilibrium. When preferences are time-consistent and, in addition, households are sophisticated all three market market structures are allocationally equivalent.

Sophisticated behavior requires a lot from households, however. A more reasonable type of behavior would be "somewhat" sophisticated. Loewenstein, O'Donoghue, and Rabin (2003) assume that households do make plans that they expect to stick to. However, they assume that households mispredict their future utility function and thus will want to reconsider their plans in the future. Here, we could model these households as having the same budget constraints as sophisticated households, but with different actual utility functions in later periods. For these households, it cannot be expected that they have perfect foresight of prices and thus, the results for the naïve households in this chapter would apply. As soon as we allow at least one household not to be completely sophisticated, this chapter shows that the degree of market completeness matters. 


\subsection{Appendix}

Proof of Theorem 6.5

Let $\left(p^{*}, q^{*}, x^{*}, y^{*}\right)$ be a naïve SC equilibrium. Consider some $s, s^{\prime} \in S$ and $\tau, t \in T$ with $\tau>t$ and $s^{\prime}(t)=s(t)$. Since the economy is locally non-satiated, it is evident that $p_{s^{\prime}(\tau) \mid s(t)}^{*} \neq 0$. Let $l$ be a commodity such that $p_{s^{\prime}(\tau), l \mid s(t)}^{*} \neq 0$.

Suppose there is $\tilde{l} \in L$ such that there is no $\mu \in \mathbb{R}$ with both

$$
\begin{aligned}
& \mu p_{s^{\prime}(\tau), l \mid s(t)}^{*}=q_{s^{\prime}(\tau), l \mid s(t)}^{*} \text { and } \\
& \mu p_{s^{\prime}(\tau), \tilde{l} \mid s(t)}^{*}=q_{s^{\prime}(\tau), \tilde{l} s(t)}^{*} .
\end{aligned}
$$

Then it follows that

$$
p_{s^{\prime}(\tau), l \mid s(t)}^{*} q_{s^{\prime}(\tau), \tilde{l} \mid s(t)}^{*} \neq p_{s^{\prime}(\tau), \tilde{l} \mid s(t)}^{*} q_{s^{\prime}(\tau), l \mid s(t)}^{*} .
$$

Let $h$ be a household such that $\succeq^{h, s(t)}$ is locally non-satiated in $s^{\prime}(\tau-1)$. Case 1: Assume that

$$
p_{s^{\prime}(\tau), l \mid s(t)}^{*} q_{s^{\prime}(\tau), \tilde{l} \mid s(t)}^{*}>p_{s^{\prime}(\tau), \tilde{l} \mid s(t)}^{*} q_{s^{\prime}(\tau), l \mid s(t)}^{*} .
$$

Now consider the vector $\Delta y_{. \mid s(t)}^{h}$ defined by

$$
\Delta y_{s^{\prime \prime}\left(t^{\prime}\right), l^{\prime} \mid s(t)}^{h}= \begin{cases}p_{s^{\prime}(\tau) \tilde{l} \mid s(t)}^{*} & \text { if } s^{\prime \prime}\left(t^{\prime}\right)=s^{\prime}(\tau) \text { and } l^{\prime}=l, \\ -p_{s^{\prime}(\tau), l \mid s(t)}^{*} & \text { if } s^{\prime \prime}\left(t^{\prime}\right)=s^{\prime}(\tau) \text { and } l^{\prime}=\tilde{l}, \\ 0 & \text { otherwise. }\end{cases}
$$

Then

$$
p_{s^{\prime}(\tau) \mid s(t)}^{*} \Delta y_{s^{\prime}(\tau) \mid s(t)}^{h}=0,
$$

i.e. in state $s(t)$ household $h$ could plan to buy $\Delta y_{\cdot \mid s(t)}^{h}$ in addition to $y_{\cdot \mid s(t)}^{* h}$, without changing income in state $s^{\prime}(\tau)$. Moreover,

$$
q_{s^{\prime}(\tau) \mid s(t)}^{*} \Delta y_{s^{\prime}(\tau) \mid s(t)}^{h}=q_{s^{\prime}(\tau), l \mid s(t)}^{*} p_{s^{\prime}(\tau), \tilde{l} \mid s(t)}^{*}-q_{s^{\prime}(\tau), \tilde{l} \mid s(t)}^{*} p_{s^{\prime}(\tau), l \mid s(t)}^{*}<0 .
$$

Therefore, buying $\Delta y_{\cdot \mid s(t)}^{h}$ in addition to $y_{\cdot \mid s(t)}^{* h}$ decreases expected expenditures in state $s^{\prime}(\tau-1)$, while not changing income in any other state.

By local non-satiation this contradicts $\left(p^{*}, q^{*}, x^{*}, y^{*}\right)$ being a naïve SC equilibrium.

Case 2: Assume that

$$
p_{s^{\prime}(\tau), l \mid s(t)}^{*} q_{s^{\prime}(\tau), \tilde{l} \mid s(t)}^{*}<p_{s^{\prime}(\tau), \tilde{l} \mid s(t)}^{*} q_{s^{\prime}(\tau), l \mid s(t)}^{*} .
$$


This case follows by repeating the argument of Case 1, replacing $\Delta y_{\left.\cdot\right|_{s}(t)}^{h}$ by $-\Delta y_{\cdot \mid s(t)}^{h}$.

It follows that for any $\tilde{l} \in L, \mu=q_{s^{\prime}(\tau), l \mid s(t)}^{*} / p_{s^{\prime}(\tau), l \mid s(t)}^{*}$ satisfies $\mu p_{s^{\prime}(\tau), \tilde{l} \mid s(t)}^{*}=$ $q_{s^{\prime}(\tau), \tilde{l} \mid s(t)}^{*}$

\section{Proof of TheOREM 6.8}

Let $\left(p^{*}, x^{*}\right)$ be an AD equilibrium. We define $q_{\cdot \mid s(t)}^{*}=p_{t+1, T \mid s(t)}^{*}$ for every $s(t)$. By local non-satiation of the economy we know that for every $s, s^{\prime} \in S, t, \tau \in T$ with $\tau>t$ and $s^{\prime}(t)=s(t)$ there is an $l_{s^{\prime}(\tau) \mid s(t)}$ with $p_{s^{\prime}(\tau), l_{s^{\prime}(\tau) \mid s(t)} \mid s(t)} \neq 0$. For every $s(t)$, we define $y_{\cdot \mid s(t)}^{* h}$ recursively as follows. Start by setting, for every $h \in H$ and every $s^{\prime} \in S$ with $s^{\prime}(t)=s(t)$,

$$
y_{s^{\prime}(T), l_{s^{\prime}(T) \mid s(t)}^{* h} \mid s(t)}^{* h}=\frac{p_{s^{\prime}(T) \mid s(t)}^{*}\left(x_{s^{\prime}(T) \mid s(t)}^{* h}-e_{s^{\prime}(T) \mid s(t)}^{h}\right)}{p_{s^{\prime}(T), l_{s^{\prime}(T) \mid s(t)} \mid s(t)}^{*}}+e_{s^{\prime}(T), l_{s^{\prime}(T) \mid s(t)}}^{h} \mid s(t),
$$

and $y_{s^{\prime}(T), l \mid s(t)}^{* h}=e_{s^{\prime}(T), l \mid s(t)}^{h}$ for every $l \neq l_{s^{\prime}(T) \mid s(t)}$. Then, continue by setting, for every $h \in H, t<\tau<T, s^{\prime} \in S$ with $s^{\prime}(t)=s(t)$,

$$
\begin{gathered}
y_{s^{\prime}(\tau), l_{s^{\prime}(\tau) \mid s(t)}^{* h} \mid s(t)}=\frac{p_{s^{\prime}(\tau) \mid s(t)}^{*}\left(x_{s^{\prime}(\tau) \mid s(t)}^{* h}-e_{s^{\prime}(\tau) \mid s(t)}^{h}\right)}{p_{s^{\prime}(\tau), l_{s^{\prime}(\tau) \mid s(t)}^{*} \mid s(t)}^{h}}+e_{s^{\prime}(\tau), l_{s^{\prime}(\tau) \mid s(t)} \mid s(t)}^{h} \\
+\frac{\sum_{s^{\prime}(\tau+1) \in s^{\prime+}(\tau)}\left(p_{s^{\prime}(\tau+1) \mid s(t)}^{*} y_{s^{\prime}(\tau+1) \mid s(t)}^{* h}-p_{s^{\prime}(\tau+1) \mid s(t)}^{*} e_{s^{\prime}(\tau+1) \mid s(t)}^{h}\right)}{p_{s^{\prime}(\tau), l_{s^{\prime}(\tau) \mid s(t)}^{*} \mid s(t)}^{h}}
\end{gathered}
$$

and $y_{s^{\prime}(\tau), l \mid s(t)}^{* h}=e_{s^{\prime}(\tau), l \mid s(t)}^{h}$ for every $l \neq l_{s^{\prime}(\tau) \mid s(t)}$.

Then it holds that

$$
p_{s^{\prime}(T) \mid s(t)}^{*} y_{s^{\prime}(T) \mid s(t)}^{* h}=p_{s^{\prime}(T) \mid s(t)}^{*} x_{s^{\prime}(T) \mid s(t)}^{* h}
$$

for all $s, s^{\prime}, t, h$, and

$$
\begin{aligned}
p_{s^{\prime}(\tau) \mid s(t)}^{*} x_{s^{\prime}(\tau) \mid s(t)}^{* h}+ & \sum_{s^{\prime}(\tau+1) \in s^{\prime+}(\tau)} p_{s^{\prime}(\tau+1) \mid s(t)}^{*} y_{s^{\prime}(\tau+1) \mid s(t)}^{* h}= \\
& p_{s^{\prime}(\tau) \mid s(t)}^{*} y_{s^{\prime}(\tau) \mid s(t)}^{* h}+\sum_{s^{\prime}(\tau+1) \in s^{\prime}(\tau)} p_{s^{\prime}(\tau+1) \mid s(t)}^{*} e_{s^{\prime}(\tau+1) \mid s(t)}^{h} .
\end{aligned}
$$

for all $s, s^{\prime}, h, t \leq \tau<T$.

We claim that $\left(p^{*}, q^{*}, x^{*}, y^{*}\right)$ is a naïve sequentially complete equilibrium. 
By summing the budget constraints over all households, using that

$$
\sum_{h \in H} x_{\cdot \mid s(t)}^{* h}=\sum_{h \in H} e_{\cdot \mid s(t)}^{h},
$$

we obtain, for every $s, t$,

$$
\sum_{h \in H} y_{\cdot \mid s(t)}^{* h}=\sum_{h \in H} e_{\cdot \mid s(t+1)}^{h}
$$

It therefore holds that conditions (b) and (c) of the definition of a naïve sequentially complete equilibrium are satisfied. It only remains to be checked that condition (a) is satisfied as well.

Consider any $t, s, h$, and any

$$
\left(x_{\cdot \mid s(t)}^{h}, y_{\cdot \mid s(t)}^{h}\right) \in{ }^{n} \bar{\gamma}_{s(t)}^{h}\left(p_{\cdot \mid s(t)}^{*}, q_{\cdot \mid s(t)}^{*}, y_{s(t) \mid s(t-1)}^{* h}, e_{t+1, T \mid s(t)}^{h}\right) .
$$

By summing up budget constraints, we have that

$$
\begin{aligned}
p_{\cdot \mid s(t)}^{*} x_{\cdot \mid s(t)}^{h}+ & p_{t+1, T \mid s(t)}^{*} y_{t+1, T \mid s(t)}^{h} \leq \\
& p_{s(t) \mid s(t)}^{*} y_{s(t) \mid s(t-1)}^{* h}+p_{t+1, T \mid s(t)}^{*} e_{t+1, T \mid s(t)}^{h}+p_{t+1, T \mid s(t)}^{*} y_{t+1, T \mid s(t)}^{h}
\end{aligned}
$$

so,

$$
p_{\cdot \mid s(t)}^{*} x_{\cdot \mid s(t)}^{h} \leq p_{s(t) \mid s(t)}^{*} y_{s(t) \mid s(t-1)}^{* h}+p_{t+1, T \mid s(t)}^{*} e_{t+1, T \mid s(t)}^{h} .
$$

In addition, we have

$$
\begin{aligned}
p_{s(\tau) \mid s(\tau)}^{*} x_{s(\tau) \mid s(\tau)}^{* h}+ & \sum_{s(\tau+1) \in s^{+}(\tau)} p_{s(\tau+1) \mid s(\tau)}^{*} y_{s(\tau+1) \mid s(\tau)}^{* h} \leq \\
& p_{s(\tau) \mid s(\tau)}^{*} y_{s(\tau) \mid s(\tau-1)}^{* h}+\sum_{s(\tau+1) \in s^{+}(\tau)} p_{s(\tau+1) \mid s(\tau)}^{*} e_{s(\tau+1) \mid s(\tau)}^{h}
\end{aligned}
$$

for every $\tau<t$.

By summing all these and by keeping in mind that $x^{*}$ is a time-consistent allocation, we find that $x_{\cdot \mid 1}^{* h} \prec x_{\cdot \mid s(t)}^{h} \in \breve{\gamma}_{1}^{h}\left(p_{\cdot \mid 1}^{*}, e_{\cdot \mid 1}^{h}\right)$.

Now suppose that there are $t, s, h$ with

$$
\left(x_{\cdot \mid s(t)}^{* h}, y_{\cdot \mid s(t)}^{* h}\right) \notin^{n} \bar{\delta}_{s(t)}^{h}\left(p_{\cdot \mid s(t)}^{*}, q_{\cdot \mid s(t)}^{*}, y_{s(t) \mid s(t-1)}^{* h}, e_{t+1, T \mid s(t)}^{h}\right) .
$$

Then there must be $\left(\hat{x}_{\cdot \mid s(t)}^{h}, \hat{y}_{\cdot \mid s(t)}^{h}\right) \in{ }^{n} \bar{\gamma}_{s(t)}^{h}\left(p_{\cdot \mid s(t)}^{*}, q_{\cdot \mid s(t)}^{*}, y_{s(t) \mid s(t-1)}^{* h}, e_{t+1, T \mid s(t)}^{h}\right)$ with $\hat{x}_{\cdot \mid s(t)}^{h} \succ^{h, s(t)} x_{\cdot \mid s(t)}^{* h}$. But then, by time-consistency of preferences, $x_{\cdot \mid 1}^{* h}\left\langle\hat{x}_{\cdot \mid s(t)}^{h} \succ^{h, 1}\right.$ 
$x_{\cdot \mid 1}^{* h}$. By the discussion above we also have $x_{\cdot \mid 1}^{* h}\left\langle\hat{x}_{\cdot \mid s(t)}^{h} \in \breve{\gamma}_{1}^{h}\left(p_{\cdot \mid 1}^{*}, e_{\cdot \mid 1}^{h}\right)\right.$. So $x_{\cdot \mid 1}^{* h} \notin$ $\breve{\delta}_{1}^{h}\left(p_{\cdot \mid 1}^{*}, e_{\cdot \mid 1}^{h}\right)$, a contradiction to the fact that $\left(p^{*}, x^{*}\right)$ is an $\mathrm{AD}$ equilibrium.

\section{Proof of Theorem 6.9}

Define $q^{*}$ and $y^{*}$ as in Theorem 6.8. We only need to verify whether condition (a) of the definition of a sophisticated SC equilibrium is satisfied. By the same argument as in Theorem 6.8 we know that for every $s$

$$
x_{\cdot \mid s(T)}^{* h} \in{ }^{s} \bar{\delta}_{s(T)}^{h}\left(p_{\cdot \mid s(T)}^{*}, y_{s(T) \mid s(T-1)}^{* h}\right) .
$$

We continue the proof by an induction argument. Assume that, for some $t$,

$$
\left(x_{\cdot \mid s(\tau)}^{* h}, y_{\cdot \mid s(\tau)}^{* h}\right) \in{ }^{s} \bar{\delta}_{s(\tau)}^{h}\left(p_{\cdot \mid s(\tau)}^{*}, q_{\cdot \mid s(\tau)}^{*}, y_{s(\tau) \mid s(\tau-1)}^{* h}, e_{\tau+1, T \mid s(\tau)}^{h}\right)
$$

for every $\tau>t$, for every $s$. Suppose that

$$
\left(x_{\cdot \mid s(t)}^{* h}, y_{\cdot \mid s(t)}^{* h}\right) \notin s \bar{\delta}_{s(t)}^{h}\left(p_{\cdot \mid s(t)}^{*}, q_{\cdot \mid s(t)}^{*}, y_{s(t) \mid s(t-1)}^{* h}, e_{t+1, T \mid s(t)}^{h}\right) .
$$

Then there must be $\left(\hat{x}_{\cdot \mid s(t)}^{h}, \hat{y}_{\cdot \mid s(t)}^{h}\right) \in{ }^{s} \bar{\gamma}_{s(t)}^{h}\left(p_{\cdot \mid s(t)}^{*}, q_{\cdot \mid s(t)}^{*}, y_{s(t) \mid s(t-1)}^{* h}, e_{t+1, T \mid s(t)}^{h}\right)$ with $\hat{x}_{\cdot \mid s(t)}^{h} \succ^{h, s(t)} x_{\cdot \mid s(t)}^{* h}$. The same argument as in the proof of Theorem 6.8 then leads to a contradiction.

\section{Proof of TheOREM 6.10}

Let $\left(p^{*}, q^{*}, x^{*}, y^{*}\right)$ be a sophisticated SC equilibrium. By Theorem 6.6 , for every $s, s^{\prime} \in S$ and every $t, \tau \in T$ with $\tau>t$ and $s^{\prime}(t)=s(t)$, there must be $\mu_{s^{\prime}(\tau) \mid s(t)}$ such that

$$
q_{s^{\prime}(\tau) \mid s(t)}^{*}=\mu_{s^{\prime}(\tau) \mid s(t)} p_{s^{\prime}(\tau) \mid s(t)}^{*} .
$$

Suppose that $\mu_{s^{\prime}(\tau) \mid 1} \leq 0$ for some $s^{\prime}(\tau)$. Then $q_{s^{\prime}(\tau) \mid 1}^{*}=\mu_{s^{\prime}(\tau) \mid 1} p_{s^{\prime}(\tau) \mid 1}^{*} \leq 0$. Thus, it would be possible to increase available income in state $s^{\prime}(\tau)$ without increasing expenditures in state $s^{\prime}(\tau-1)$. A household $h$ that, from the perspective of period 1 , is locally non-satiated in state $s^{\prime}(\tau)$, could change consumption in state $s^{\prime}(\tau)$ and have a consumption plan that is strictly preferred to the current bundle. Since preferences are time-consistent, the new consumption bundle would also be consistent with sophisticated behavior. This yields a contradiction against $x^{*}$ being an equilibrium allocation. It follows that $\mu_{s^{\prime}(\tau) \mid 1}>0$ for every $s^{\prime}(\tau)$.

Let $p_{1 \mid 1}=p_{1 \mid 1}^{*}$ and $p_{s^{\prime}(\tau) \mid 1}=p_{s^{\prime}(\tau) \mid 1}^{*} \prod_{\tau^{\prime}=2}^{\tau} \mu_{s^{\prime}\left(\tau^{\prime}\right) \mid 1}$ for every $s^{\prime} \in S$ and every $\tau>1$. Also, let $p_{\cdot \mid s(t)}=p_{s(t), T \mid 1}$ for all $t \in T, s \in S$. It holds that $p \gg 0$ and therefore that $\breve{\gamma}_{1}^{h}\left(p_{\cdot \mid 1}, e_{\cdot \mid 1}^{h}\right)$ is compact. 
Suppose that $\left(p, x^{*}\right)$ is not an AD equilibrium. Then there is $s(t)$ with $x_{\cdot \mid s(t)}^{* h} \notin$ $\breve{\delta}_{s(t)}^{h}\left(p_{\cdot \mid s(t)}, x_{s(t), T \mid s(t-1)}^{* h}\right)$. Since we have $x_{\cdot \mid s(t)}^{* h}=x_{s(t), T \mid s(t-1)}^{* h}$ for every $t>1$, it should hold that $s(t)=1$. We know that $x_{\cdot \mid 1}^{* h} \in \breve{\gamma}_{1}^{h}\left(p_{\cdot \mid 1}, e_{\cdot \mid 1}^{h}\right)$. So there is $\bar{x}_{\cdot \mid 1}^{h}$ with $\bar{x}_{\cdot \mid 1}^{h} \in \breve{\gamma}_{1}^{h}\left(p_{\cdot \mid 1}, e_{\cdot \mid 1}^{h}\right)$ and $\bar{x}_{\cdot \mid 1}^{h} \succ^{h, 1} x_{\cdot \mid 1}^{* h}$. Since $\succeq^{h, 1}$ is acyclic and has open lower sections and $\breve{\gamma}_{1}^{h}\left(p_{\cdot \mid 1}, e_{\cdot \mid 1}^{h}\right)$ is compact, according to Bergstrom (1975) there exists a maximum on $\breve{\gamma}_{1}^{h}\left(p_{\cdot \mid 1}, e_{\cdot \mid 1}^{h}\right)$ with respect to $\succeq^{h, 1}$. Without loss of generality, $\bar{x}_{\cdot \mid 1}^{h}$ equals that maximum. We define $\bar{x}_{\cdot \mid s(t)}^{h}=\bar{x}_{s(t), T \mid 1}^{h}$ for every $s(t)$. By the same argument as in the proof of Theorem 6.8, there must be $\bar{y}$ with $\left(\bar{x}_{\cdot \mid 1}^{h}, \bar{y}_{\cdot \mid 1}^{h}\right) \in{ }^{n} \bar{\gamma}_{1}^{h}\left(p_{\cdot \mid 1}, p_{2, T \mid 1}, e_{\cdot \mid 1}^{h}\right)$ and $\bar{y}_{s(t+1), T \mid s(t)}^{h}=\bar{y}_{s(t+1), T \mid 1}^{h}$ for every $s(t)$. Moreover, from the opportunity sets, it follows that whenever there is $y_{1}^{h}$ with $\left(x_{1}^{h}, y_{1}^{h}\right) \in{ }^{n} \bar{\gamma}_{1}^{h}\left(p_{\cdot \mid 1}, p_{2, T \mid 1}, e_{\cdot \mid 1}^{h}\right)$, then there is ${y^{\prime}}_{1}^{h}$ with $\left(x_{1}^{h}, y_{1}^{\prime h}\right) \in{ }^{n} \bar{\gamma}_{1}^{h}\left(p_{\cdot \mid 1}^{*}, q_{\cdot \mid 1}^{*}, e_{\cdot \mid 1}^{h}\right)$ and vice versa.

Then, by definition of $\bar{x}_{\cdot \mid 1}^{h}$ and the fact that $\bar{x}_{\cdot \mid 1}^{h} \succ^{h, 1} x_{\cdot \mid 1}^{* h}$, there is $s(t)$ such that

$$
\left(\bar{x}_{\cdot \mid s(t)}^{h}, y_{\cdot \mid s(t)}^{h}\right) \notin s \bar{\delta}_{s(t)}^{h}\left(p_{\cdot \mid s(t)}^{*}, q_{\cdot \mid s(t)}^{*}, \bar{y}_{s(t) \mid s(t-1)}^{h}, e_{t+1, T \mid s(t)}^{h}\right)
$$

for any choice of $y_{\cdot \mid s(t)}^{h}$. Two cases can be distinguished.

Case 1:

There exists $y_{\cdot \mid s(t)}^{h}$ with $\left(\bar{x}_{\cdot \mid s(t)}^{h}, y_{\cdot \mid s(t)}^{h}\right) \in{ }^{s} \bar{\gamma}_{s(t)}^{h}\left(p_{\cdot \mid s(t)}^{*}, q_{\cdot \mid s(t)}^{*}, \bar{y}_{s(t) \mid s(t-1)}^{h}, e_{t+1, T \mid s(t)}^{h}\right)$. Then there must be $\left(\hat{x}_{\cdot \mid s(t)}^{h}, \tilde{y}_{\cdot \mid s(t)}^{h}\right) \in s \bar{\gamma}_{s(t)}^{h}\left(p_{\cdot \mid s(t)}^{*}, q_{\cdot \mid s(t)}^{*}, \bar{y}_{s(t) \mid s(t-1)}^{h}, e_{t+1, T \mid s(t)}^{h}\right)$ with $\hat{x}_{\cdot \mid s(t)}^{h} \succ^{h, s(t)} \bar{x}_{\cdot \mid s(t)}^{h}$. By time-consistency of preferences, it follows that $\bar{x}_{\cdot \mid 1}^{h}$ ? $\hat{x}_{\cdot \mid s(t)}^{h} \succ^{h, 1} \bar{x}_{\cdot \mid 1}^{h}$. Also, it can be checked that $\bar{x}_{\cdot \mid 1}^{h} \prec \hat{x}_{\cdot \mid s(t)}^{h} \in \breve{\gamma}_{1}^{h}\left(p_{\cdot \mid 1}, e_{\cdot \mid 1}^{h}\right)$. This contradicts the definition of $\bar{x}_{\cdot \mid 1}^{h}$. Thus, case 1 is not possible.

Case 2:

There does not exist $y_{\cdot \mid s(t)}^{h}$ with $\left(\bar{x}_{\cdot \mid s(t)}^{h}, y_{\cdot \mid s(t)}^{h}\right) \in s \bar{\gamma}_{s(t)}^{h}\left(p_{\cdot \mid s(t)}^{*}, q_{\cdot \mid s(t)}^{*}, \bar{y}_{s(t) \mid s(t-1)}^{h}, e_{t+1, T \mid s(t)}^{h}\right)$.

Since $\bar{x}_{\cdot \mid 1}^{h} \in \breve{\gamma}_{1}^{h}\left(p_{\cdot \mid 1}, e_{\cdot \mid 1}^{h}\right)$ and relative prices do not change over time and are correctly anticipated, this can only happen if there is $s\left(t^{\prime}\right)$ with $t^{\prime}>t$ and

$$
\left(\bar{x}_{\cdot \mid s\left(t^{\prime}\right)}^{h}, \tilde{y}_{\cdot \mid s\left(t^{\prime}\right)}^{h}\right) \notin s \bar{\delta}_{s\left(t^{\prime}\right)}^{h}\left(p_{\cdot \mid s\left(t^{\prime}\right)}^{*}, q_{\cdot \mid s\left(t^{\prime}\right)}^{*}, \bar{y}_{s\left(t^{\prime}\right) \mid s\left(t^{\prime}-1\right)}^{h}, e_{t^{\prime}+1, T \mid s\left(t^{\prime}\right)}^{h}\right)
$$

for every $\tilde{y}_{\cdot \mid s\left(t^{\prime}\right)}^{h}$.

Next, as before, for $s\left(t^{\prime}\right)$ we can distinguish two cases. We repeat this reasoning until we end up at the last period. For the last period, we can no longer distinguish two cases. In the last period, only case 1 can happen. This again leads to a contradiction by a similar reasoning as before. 


\section{Proof of Theorem 6.13}

Consider some $s, s^{\prime} \in S$ and $t, \tau \in T$ with $\tau>t$ and $s^{\prime}(t)=s(t)$. Since the economy is locally non-satiated, it holds that $p_{s^{\prime}(\tau) \mid s(t)}^{*} \neq 0$. Let $l$ be a commodity such that $p_{s^{\prime}(\tau), l \mid s(t)}^{*} \neq 0$.

Apart from the fact that we have more cases, the remainder of the proof is analogous to the proof of Theorem 6.5. Suppose that there is no $\mu \in \mathbb{R}$ with both

$$
\begin{aligned}
& \mu p_{s^{\prime}(\tau), l \mid s(t)}^{*}=q_{\left(s^{\prime}(\tau), l \mid s^{\prime}(\tau-1)\right) \mid s(t)}^{*}, \\
& \mu p_{s^{\prime}(\tau), \tilde{l} \mid s(t)}^{*}=q_{\left(s^{\prime}(\tau), \tilde{l} \mid s^{\prime}(\tau-1)\right) \mid s(t)}^{*}
\end{aligned}
$$

or that there is no $\mu$ with both

$$
\begin{aligned}
\mu p_{s^{\prime}(\tau), l \mid s(t)}^{*} & =q_{\left(s^{\prime}(\tau), l \mid s^{\prime}(\tau-1)\right) \mid s(t)}^{*} \text { and } \\
\mu q_{\left(s^{\prime \prime}(\tilde{\tau}), \tilde{l} \mid s^{\prime}(\tau)\right) \mid s(t)}^{*} & =q_{\left(s^{\prime \prime}(\tilde{\tau}), \tilde{l} \mid s^{\prime}(\tau-1)\right) \mid s(t)}^{*} .
\end{aligned}
$$

Next a contradiction can be obtained and the proof can be finished in a similar way as in the proof of Theorem 6.5.

\section{Proof of TheOREM 6.14}

Let prices be as described in the theorem. First of all, we need one observation. Consider

$$
\left(x_{\cdot \mid s(t)}^{h}, y_{\cdot \mid s(t)}^{h}\right) \in{ }^{n} \gamma_{s(t)}^{h}\left(p_{\cdot \mid s(t)}^{*}, q_{\cdot \mid s(t)}^{*}, y_{(\cdot \mid s(t-1)) \mid s(t-1)}^{* h}\right)
$$

Then we have

$p_{s^{\prime}(\tau) \mid s(t)}^{*} x_{s^{\prime}(\tau) \mid s(t)}^{h}+p_{s^{\prime}(\tau), T \mid s(t)}^{*} y_{\left(\tau+1, T \mid s^{\prime}(\tau)\right) \mid s(t)}^{h} \leq p_{s^{\prime}(\tau), T \mid s(t)}^{*} y_{\left(s^{\prime}(\tau), T \mid s^{\prime}(\tau-1)\right) \mid s(t)}^{h}$ for every $s^{\prime}, \tau \geq t$. By adding these over all $s^{\prime}(\tau)$ with $\tau \geq t$ and $s^{\prime}(t)=s(t)$, we get

$$
p_{\cdot \mid s(t)}^{*} x_{\cdot \mid s(t)}^{h} \leq p_{\cdot \mid s(t)}^{*} y_{(s(t), T \mid s(t-1)) \mid s(t-1)}^{h} .
$$

Thus, we also have

$$
p_{\cdot \mid s^{\prime}(t)}^{*} x_{\cdot \mid s^{\prime}(t)}^{* h} \leq p_{\cdot \mid s^{\prime}(t)}^{*} y_{\left(s^{\prime}(t-1), T \mid s^{\prime}(t-1)\right) \mid s^{\prime}(t)}^{* h}
$$

for every $s^{\prime}$. Similarly, for all $\tau<t$, we know that

$$
p_{s(\tau) \mid s(\tau)}^{*} x_{s(\tau) \mid s(\tau)}^{* h}+p_{\tau+1, T \mid s(\tau)}^{*} y_{(\tau+1, T \mid s(\tau)) \mid s(\tau)}^{* h} \leq p_{\cdot \mid s(\tau)}^{*} y_{(s(\tau), T \mid s(\tau-1)) \mid s(\tau-1)}^{* h}
$$


by definition of $y^{*}$. Using that $p_{s(\tau), T \mid s(t)}^{*}=p_{\cdot \mid s(\tau)}^{*}$, and taking the sum of equation (6.2) for all $s^{\prime}(t) \neq s(t)$, equation (6.1) and equation (6.3) for all $s(\tau)$ with $\tau<t$, we obtain $x_{\cdot \mid 1}^{* h} 2 x_{\cdot \mid s(t)}^{h} \in \breve{\gamma}_{1}^{h}\left(p_{\cdot \mid 1}^{*}, e_{\cdot \mid 1}^{h}\right)$.

With this observation in mind, we can continue the proof. Since $\left(p^{*}, x^{*}\right)$ is an $\mathrm{AD}$ equilibrium and by definition of $y^{*}$, it follows immediately that conditions (b) and (c) of the definition of a naïve complete equilibrium are satisfied. It remains to be shown that condition (a) is satisfied as well. Suppose there are $t, s(t), h$ with $\left(x_{\cdot \mid s(t)}^{* h}, y_{\cdot \mid s(t)}^{* h}\right) \notin^{n} \delta_{s(t)}^{h}\left(p_{\cdot \mid s(t)}^{*}, q_{\cdot \mid s(t)}^{*}, y_{(\cdot \mid s(t-1)) \mid s(t-1)}^{* h}\right)$. Then there must be

$$
\left(\hat{x}_{\cdot \mid s(t)}^{h}, y_{\cdot \mid s(t)}^{h}\right) \in{ }^{n} \gamma_{s(t)}^{h}\left(p_{\cdot \mid s(t)}^{*}, q_{\cdot \mid s(t)}^{*}, y_{(\cdot \mid s(t-1)) \mid s(t-1)}^{* h}\right)
$$

with $\hat{x}_{\cdot \mid s(t)}^{h} \succ^{h, s(t)} x_{\cdot \mid s(t)}^{* h}$. But then, by time-consistency of preferences, $x_{\cdot \mid 1}^{* h} 2$ $\hat{x}_{\cdot \mid s(t)}^{h} \succ^{h, 1} x_{\cdot \mid 1}^{* h}$. By our observation, it follows that $x_{\cdot \mid 1}^{* h}\left\langle\hat{x}_{\cdot \mid s(t)}^{h} \in \breve{\gamma}_{1}^{h}\left(p_{\cdot \mid 1}^{*}, e_{\cdot \mid 1}^{h}\right)\right.$. So $x_{\cdot \mid 1}^{* h} \notin \breve{\delta}_{1}^{h}\left(p_{\cdot \mid 1}^{*}, e_{\cdot \mid 1}^{h}\right)$, contradicting that $\left(p^{*}, x^{*}\right)$ is an $\mathrm{AD}$ equilibrium.

\section{Proof of TheORem 6.15}

First, of all, by definition of $p^{*}, q^{*}, x^{*}$, and $y^{*}$, it follows immediately that conditions (b), (c), (d), (e), (f), and (g) of the definition of a sophisticated complete equilibrium are satisfied. It remains to be shown that condition (a) is satisfied as well.

By the same argument as used in the proof of Theorem 6.14, it holds that

$$
\left(x_{\cdot \mid s(T)}^{* h}, y_{\cdot \mid s(T)}^{* h}\right) \in{ }^{s} \delta_{s(T)}^{h}\left(p_{\cdot \mid s(T)}^{*}, y_{\cdot \mid s(T-1)}^{* h}\right) .
$$

We proceed with an induction argument. Let $s \in S, t \in T$. Assume that

$$
\left(x_{\cdot \mid s(t+1)}^{* h}, y_{\cdot \mid s(t+1)}^{* h}\right) \in{ }^{s} \delta_{s(t+1)}^{h}\left(p_{\cdot \mid s(t+1)}^{*}, q_{\cdot \mid s(t+1)}^{*}, y_{(\cdot \mid s(t)) \mid s(t)}^{* h}\right)
$$

for every $s(t+1) \in s^{+}(t)$. Suppose that

$$
\left(x_{\cdot \mid s(t)}^{* h}, y_{\cdot \mid s(t)}^{* h}\right) \notin s \delta_{s(t)}^{h}\left(p_{\cdot \mid s(t)}^{*}, q_{\cdot \mid s(t)}^{*}, y_{(\cdot \mid s(t-1)) \mid s(t-1)}^{* h}\right) .
$$

Then there must be

$$
\left(\hat{x}_{\cdot \mid s(t)}^{h}, \hat{y}_{\cdot \mid s(t)}^{h}\right) \in{ }^{s} \gamma_{s(t)}^{h}\left(p_{\cdot \mid s(t)}^{*}, q_{\cdot \mid s(t)}^{*}, y_{(\cdot \mid s(t-1)) \mid s(t-1)}^{* h}\right)
$$

with $\hat{x}_{\cdot \mid s(t)}^{h} \succ^{h, s(t)} x_{\cdot \mid s(t)}^{* h}$. But then, by time-consistency of preferences $x_{\cdot \mid 1}^{* h}\left\langle\hat{x}_{\cdot \mid s(t)}^{h} \succ^{h, 1}\right.$ $x_{\cdot \mid 1}^{* h}$. By the same argument as in the proof of Theorem 6.14 we also have $x_{\cdot \mid 1}^{* h}$ ? $\hat{x}_{\cdot \mid s(t)}^{h} \in \breve{\gamma}_{1}^{h}\left(p_{\cdot \mid 1}^{*}, e_{\cdot \mid 1}^{h}\right)$. This contradicts $\left(p^{*}, x^{*}\right)$ being an AD equilibrium. 
Proof OF TheOREM 6.17

This proof is analogous to the proof for the sophisticated SC equilibrium in Theorem 6.10 .

\section{Proof of TheOREM 6.18}

Since $\left(p^{*}, q^{*}, x^{*}, y^{*}\right)$ is a naïve $\mathrm{SC}$ equilibrium, it holds that

$$
\left(x_{\cdot \mid s(t)}^{* h}, y_{\cdot \mid s(t)}^{* h}\right) \in{ }^{n} \bar{\delta}_{s(t)}^{h}\left(p_{\cdot \mid s(t)}^{*}, q_{\cdot \mid s(t)}^{*}, y_{s(t) \mid s(t-1)}^{* h}, e_{t+1, T \mid s(t)}^{h}\right) .
$$

For $s(t), s^{\prime}(\tau)$ with $\tau>t$, we define $\mu_{s^{\prime}(\tau) \mid s(t)}$ as the unique real number satisfying $q_{s^{\prime}(\tau) \mid s(t)}^{*}=\mu_{s^{\prime}(\tau) \mid s(t)} p_{s^{\prime}(\tau) \mid s(t)}^{*}$, see Theorem 6.5.

Next, we define $p_{s(t) \mid s(t)}=p_{s(t) \mid s(t)}^{*}, p_{s^{\prime}(\tau) \mid s(t)}^{*}=p_{s^{\prime}(\tau) \mid s(t)}^{*} \prod_{\tau^{\prime}=t+1}^{\tau} \mu_{s^{\prime}\left(\tau^{\prime}\right) \mid s(t)}$, $q_{\left(s^{\prime}(\tau), T \mid s^{\prime}(\tau-1)\right) \mid s(t)}=p_{s^{\prime}(\tau), T \mid s(t)}$.

Let $y_{\left(s^{\prime}(\tau+1) \mid s^{\prime}(\tau)\right) \mid s(t)}^{h}=y_{s^{\prime}(\tau+1) \mid s(t)}^{* h}$ and $y_{\left(s^{\prime}(\tau+2), T \mid s^{\prime}(\tau)\right) \mid s(t)}^{h}=e_{\cdot \mid s^{\prime}(\tau+2)}^{h}$ for every $\tau \geq t$ and every $s^{\prime}$ with $s^{\prime}(t)=s(t)$. Then

$$
\left(x_{\cdot \mid s(t)}^{* h}, y_{\cdot \mid s(t)}^{h}\right) \in{ }^{n} \gamma_{s(t)}^{h}\left(p_{\cdot \mid s(t)}, q_{\cdot \mid s(t)}, y_{s(t) \mid s(t-1)}^{* h}, e_{t+1, T \mid s(t)}^{h}\right) .
$$

Suppose that $\left(x_{\cdot \mid s(t)}^{* h}, y_{\cdot \mid s(t)}^{h}\right) \notin n \delta_{s(t)}^{h}\left(p_{\cdot \mid s(t)}, q_{\cdot \mid s(t)}, y_{s(t) \mid s(t-1)}^{* h}, e_{t+1, T \mid s(t)}^{h}\right)$. Then there must be $\left(x_{\cdot \mid s(t)}^{h}, \tilde{y}_{\cdot \mid s(t)}^{h}\right) \in{ }^{n} \gamma_{s(t)}^{h}\left(p_{\cdot \mid s(t)}, q_{\cdot \mid s(t)}, y_{s(t) \mid s(t-1)}^{* h}, e_{t+1, T \mid s(t)}^{h}\right)$ with $x_{\cdot \mid s(t)}^{h}$ $\succ^{h, s(t)} x_{\cdot \mid s(t)}^{* h}$. As in the proof of Theorem 6.8 it follows that there is $\widehat{y}_{\cdot \mid s(t)}^{h}$ with

$$
\left(x_{\cdot \mid s(t)}^{h}, \widehat{y}_{\cdot \mid s(t)}^{h}\right) \in{ }^{n} \bar{\gamma}_{s(t)}^{h}\left(p_{\cdot \mid s(t)}^{*}, q_{\cdot \mid s(t)}^{*}, y_{s(t) \mid s(t-1)}^{* h}, e_{t+1, T \mid s(t)}^{h}\right) .
$$

This then contradicts the fact that

$$
\left(x_{\cdot \mid s(t)}^{* h}, y_{\cdot \mid s(t)}^{* h}\right) \in{ }^{n} \bar{\delta}_{s(t)}^{h}\left(p_{\cdot \mid s(t)}^{*}, q_{\cdot \mid s(t)}^{*}, y_{s(t) \mid s(t-1)}^{* h}, e_{t+1, T \mid s(t)}^{h}\right) .
$$

\section{Proof of TheOREM 6.20}

Step 1

Consider $p \in P, q \in Q, \bar{q} \in \bar{Q}$, and, for every $s, s^{\prime}$, for every $\tau, t$ with $\tau \geq t$ and $s^{\prime}(t)=s(t), \mu_{s^{\prime}(\tau) \mid s(t)} \in \mathbb{R}$ such that

$$
\begin{aligned}
p_{s(\tau), T \mid s(t)} & =p_{\cdot \mid s(\tau)}, \\
\bar{q}_{s^{\prime}(\tau) \mid s(t)} & =\mu_{s^{\prime}(\tau) \mid s(t)} p_{s^{\prime}(\tau) \mid s(t)} \\
q_{\left(s^{\prime}(\tau), T \mid s^{\prime}(\tau-1)\right) \mid s(t)} & =\mu_{s^{\prime}(\tau) \mid s(t)}\left(p_{s^{\prime}(\tau) \mid s(t)}, q_{\left(\cdot \mid s^{\prime}(\tau)\right) \mid s(t)}\right), \\
p_{s^{\prime}(\tau) \mid s(t)} & \neq 0 .
\end{aligned}
$$


For every $s(\tau)$, for every $y_{\cdot \mid s(\tau-1)}^{h} \in Y_{\cdot \mid s(\tau-1)}^{h}$, for every $\bar{y}_{\cdot \mid s(\tau-1)}^{h} \in \bar{Y}_{\cdot \mid s(\tau-1)}^{h}$, we will prove that whenever household $h$ starts with the same income in both market structures, i.e.

$$
\begin{aligned}
& p_{s(\tau) \mid s(\tau)} y_{(s(\tau) \mid s(\tau-1)) \mid s(\tau-1)}^{h} \\
& +\sum_{s^{\prime}(\tau+1) \in s^{+}(\tau)} q_{\left(s^{\prime}(\tau+1), T \mid s(\tau)\right) \mid s(\tau)} y_{\left(s^{\prime}(\tau+1), T \mid s(\tau-1)\right) \mid s(\tau-1)}^{h}= \\
& p_{s(\tau) \mid s(\tau)} \bar{y}_{s(\tau) \mid s(\tau-1)}^{h}+q_{(\tau+1, T \mid s(\tau)) \mid s(\tau)} e_{\tau+1, T \mid s(\tau)}^{h},
\end{aligned}
$$

we have that:

$$
\left(x_{\cdot \mid s(\tau)}^{h}, y_{\cdot \mid s(\tau)}^{h}\right) \in{ }^{s} \gamma_{s(\tau)}^{h}\left(p_{\cdot \mid s(\tau)}, q \cdot \mid s(\tau), y_{(\cdot \mid s(\tau-1)) \mid s(\tau-1)}^{h}\right)
$$

implies that there is $\bar{y}_{\cdot \mid s(\tau)}^{h} \in \bar{Y}_{\cdot \mid s(\tau)}^{h}$ such that

$$
\left(x_{\cdot \mid s(\tau)}^{h}, \bar{y}_{\cdot \mid s(\tau)}^{h}\right) \in{ }^{s} \bar{\gamma}_{s(\tau)}^{h}\left(p_{\cdot \mid s(\tau)}, \bar{q}_{\cdot \mid s(\tau)}, \bar{y}_{s(\tau) \mid s(\tau-1)}^{h}, e_{\tau+1, T \mid s(\tau)}^{h}\right)
$$

and vice versa. We give a proof by induction.

[Step 1a]

First of all, it is obvious that for every $\bar{y}_{s(T) \mid s(T-1)}^{h}, y_{s(T) \mid s(T-1)}^{h}$ with

$$
p_{\cdot \mid s(T)} \bar{y}_{s(T) \mid s(T-1)}^{h}=p_{\cdot \mid s(T)} y_{s(T) \mid s(T-1)}^{h}
$$

it holds that

$$
s \bar{\gamma}_{s(T)}^{h}\left(p_{\cdot \mid s(T)}, \bar{y}_{s(T) \mid s(T-1)}^{h}\right)={ }^{s} \gamma_{s(T)}^{h}\left(p_{\cdot \mid s(T)}, y_{s(T) \mid s(T-1)}^{h}\right) .
$$

[Step 1b]

Assume the result is true for every $s(\tau)$, where $\tau>t$.

Now assume that, in state $s(t)$, household $h$ starts with the same income in both market structures, i.e.

$$
\begin{aligned}
& p_{s(t) \mid s(t)} y_{(s(t) \mid s(t-1)) \mid s(t-1)}^{h} \\
& +\sum_{s^{\prime}(t+1) \in s^{+}(t)} q_{\left(s^{\prime}(t+1), T \mid s(t)\right) \mid s(t)} y_{\left(s^{\prime}(t+1), T \mid s(t-1)\right) \mid s(t-1)}^{h}= \\
& p_{s(t) \mid s(t)} \bar{y}_{s(t) \mid s(t-1)}^{h}+q_{(t+1, T \mid s(t)) \mid s(t)} e_{t+1, T \mid s(t)}^{h} .
\end{aligned}
$$

We first prove one direction of our result.

$(\Rightarrow) \quad$ Let

$$
\left(x_{\cdot \mid s(t)}^{h}, y_{\cdot \mid s(t)}^{h}\right) \in{ }^{s} \gamma_{s(t)}^{h}\left(p_{\cdot \mid s(t)}, q \cdot \mid s(t), y_{(\cdot \mid s(t-1)) \mid s(t-1)}^{h}\right)
$$


We define $\bar{y}_{\cdot \mid s(t)}^{h}$ recursively as follows. For every $s^{\prime}(\tau), s(t)$ there is a $l_{s^{\prime}(\tau) \mid s(t)}$ with $p_{s^{\prime}(\tau), l_{s^{\prime}(\tau) \mid s(t)} \mid s(t)} \neq 0$. Start by setting, for every $h \in H$,

$$
\bar{y}_{s^{\prime}(T), l_{s^{\prime}(T) \mid s(t)}^{h} \mid s(t)}^{h}=\frac{p_{s^{\prime}(T) \mid s(t)}\left(x_{s^{\prime}(T) \mid s(t)}^{h}-e_{s^{\prime}(T) \mid s(t)}^{h}\right)}{p_{s^{\prime}(T), l_{s^{\prime}(T) \mid s(t)} \mid s(t)}}+e_{s^{\prime}(T), l_{s^{\prime}(T) \mid s(t)}^{h} \mid s(t)}^{h},
$$

and $\bar{y}_{s^{\prime}(T), l \mid s(t)}^{h}=e_{s^{\prime}(T), l \mid s(t)}^{h}$ for every $l \neq l_{s^{\prime}(T) \mid s(t)}$. Then, continue by setting, for every $h \in H, t<\tau<T, s^{\prime} \in S$ with $s^{\prime}(t)=s(t)$,

$$
\begin{array}{r}
\bar{y}_{s^{\prime}(\tau), l_{s^{\prime}(\tau) \mid s(t)}^{h} \mid s(t)}^{h}=\frac{p_{s^{\prime}(\tau) \mid s(t)}\left(x_{s^{\prime}(\tau) \mid s(t)}^{h}-e_{s^{\prime}(\tau) \mid s(t)}^{h}\right)}{p_{s^{\prime}(\tau), l_{s^{\prime}(\tau) \mid s(t)} \mid s(t)}}+e_{s^{\prime}(\tau), l_{s^{\prime}(\tau) \mid s(t)}^{h} \mid s(t)}^{h} \\
+\frac{\sum_{s^{\prime}(\tau+1) \in s^{\prime}+(\tau)}\left(\bar{q}_{s^{\prime}(\tau+1) \mid s(t)} \bar{y}_{s^{\prime}(\tau+1) \mid s(t)}^{h}-\bar{q}_{s^{\prime}(\tau+1) \mid s(t)} e_{s^{\prime}(\tau+1) \mid s(t)}^{h}\right)}{p_{s^{\prime}(\tau), l_{s^{\prime}(\tau) \mid s(t)} \mid s(t)}^{h}}
\end{array}
$$

and $y_{s^{\prime}(\tau), l \mid s(t)}^{* h}=e_{s^{\prime}(\tau), l \mid s(t)}^{h}$ for every $l \neq l_{s^{\prime}(\tau) \mid s(t)}$.

Then,

$$
\begin{gathered}
p_{s(t+1) \mid s(t+1)} \bar{y}_{s(t+1) \mid s(t)}^{h} \\
+\sum_{s^{\prime}(t+2) \in s^{+}(t+1)} q_{\left(s^{\prime}(t+2), T \mid s(t+1)\right) \mid s(t)} e_{s^{\prime}(t+2), T \mid s(t)}^{h}= \\
p_{s(t+1) \mid s(t+1)} x_{s(t+1) \mid s(t)}^{h}+\sum_{s^{\prime}(t+2) \in s^{+}(t+1)} q_{\left(s^{\prime}(t+2), T \mid s(t+1)\right) \mid s(t)} x_{s^{\prime}(t+2), T \mid s(t)}^{h} .
\end{gathered}
$$

and

$$
\begin{aligned}
& p_{s(t+1) \mid s(t+1)} y_{(s(t+1) \mid s(t)) \mid s(t)}^{h} \\
& +\sum_{s^{\prime}(t+2) \in s^{+}(t+1)} q_{\left(s^{\prime}(t+2), T \mid s(t+1)\right) \mid s(t+1)} y_{\left(s^{\prime}(t+2), T \mid s(t)\right) \mid s(t)}^{h}= \\
& p_{s(t+1) \mid s(t+1)} x_{s(t+1) \mid s(t)}^{h}+\sum_{s^{\prime}(t+2) \in s^{+}(t+1)} q_{\left(s^{\prime}(t+2), T \mid s(t+1)\right) \mid s(t+1)} x_{s^{\prime}(t+2), T \mid s(t)}^{h}
\end{aligned}
$$

so it follows that

$$
\begin{aligned}
& p_{s(t+1) \mid s(t+1)} y_{(s(t+1) \mid s(t)) \mid s(t)}^{h}+ \\
& \sum_{s^{\prime}(t+2) \in s^{+}(t+1)} q_{\left(s^{\prime}(t+2), T \mid s(t+1)\right) \mid s(t+1)} y_{\left(s^{\prime}(t+2), T \mid s(t)\right) \mid s(t)}^{h}= \\
& p_{s(t+1) \mid s(t+1)} \bar{y}_{s(t+1) \mid s(t)}^{h}+\sum_{s^{\prime}(t+2) \in s^{+}(t+1)} q_{\left(s^{\prime}(t+2), T \mid s(t+1)\right) \mid s(t)} e_{s^{\prime}(t+2), T \mid s(t)}^{h} .
\end{aligned}
$$


Using our induction hypothesis, we obtain easily that

$$
\begin{aligned}
\left(x_{s(t+1), T \mid s(t)}^{h}, \bar{y}_{s^{+}(t+1), T \mid s(t)}^{h}\right) \in & \\
& s \bar{\delta}_{s(t+1)}^{h}\left(p_{s(t+1), T \mid s(t)}, \bar{q}_{s^{+}(t+1), T \mid s(t)}, \bar{y}_{s(t+1) \mid s(t)}^{h}, e_{t+2, T \mid s(t+1)}^{h}\right) .
\end{aligned}
$$

Since the income of household $h$ in state $s(t)$ is the same under both market structures, it follows that

$$
\left(x_{\cdot \mid s(t)}^{h}, \bar{y}_{\cdot \mid s(t)}^{h}\right) \in{ }^{s} \bar{\gamma}_{s(t)}^{h}\left(p_{\cdot \mid s(t)}, \bar{q}_{\cdot \mid s(t)}, \bar{y}_{s(t) \mid s(t-1)}^{h}, e_{t+1, T \mid s(t)}^{h}\right) .
$$

$(\Leftarrow) \quad$ The other direction of our result is straightforward, when following the approach used in Theorem 6.18

Step 2

Let $\left(p^{*}, q^{*}, x^{*}, y^{*}\right)$ be a sophisticated SC equilibrium. By local non-satiation we know that for all $s^{\prime}(\tau), p_{s^{\prime}(\tau) \mid 1}^{*} \neq 0$. By Theorem 6.6 there is $\mu_{s^{\prime}(\tau) \mid 1} \in \mathbb{R}$ such that

$$
q_{s^{\prime}(\tau) \mid 1}^{*}=\mu_{s^{\prime}(\tau) \mid 1} p_{s^{\prime}(\tau) \mid 1}^{*} .
$$

We define $q \in Q$ as follows.

For every $s^{\prime}(T)$,

$$
q_{\left(s^{\prime}(T) \mid s^{\prime}(T-1)\right) \mid 1}=\mu_{s^{\prime}(T) \mid 1}\left(p_{s^{\prime}(T) \mid 1}^{*}\right) .
$$

Next, recursively, for $\tau$ descending from $T-1$ to 2 , for every $s^{\prime}(\tau)$,

$$
q_{\left(s^{\prime}(\tau), T \mid s^{\prime}(\tau-1)\right) \mid 1}=\mu_{s^{\prime}(\tau) \mid 1}\left(p_{s^{\prime}(\tau) \mid 1}^{*}, q_{\left(\cdot \mid s^{\prime}(\tau)\right) \mid 1}\right) .
$$

Finally, for every $s, s^{\prime}$, for every $t, \tau$ with $\tau \geq t$ and $s^{\prime}(t)=s(t)$,

$$
q_{\left(\cdot \mid s^{\prime}(\tau)\right) \mid s(t)}=q_{\left(\cdot \mid s^{\prime}(\tau)\right) \mid 1}
$$

From the previous analysis it follows that there is a $y$ such that $\left(p^{*}, q, x^{*}, y\right)$ is a sophisticated complete equilibrium.

Step 3

Let $\left(p^{*}, q^{*}, x^{*}, y^{*}\right)$ be a sophisticated complete equilibrium. Consider any $s, s^{\prime}$, any $t, \tau$ with $\tau \geq t$ and $s^{\prime}(t)=s(t)$. By local non-satiation we know that $p_{s^{\prime}(\tau) \mid s(t)}^{*} \neq 0$. We define

$$
\bar{q}_{s^{\prime}(\tau) \mid s(t)}=q_{\left(s^{\prime}(\tau) \mid s^{\prime}(\tau-1)\right) \mid s(t)}^{*}
$$

From the previous analysis it follows that there is $y \in \bar{Y}$ such that $\left(p^{*}, \bar{q}, x^{*}, y\right)$ is a sophisticated SC equilibrium. 



\section{Arbitrage Opportunities in FRICTIONLESS MARKETS WITH SOPHISTICATED InvESTORS}

Imagine that it is possible to buy a portfolio of assets that costs nothing today and that will yield a positive payoff in the future. It is a widespread belief that, if there are no market frictions, such arbitrage opportunities cannot exist. The reason is that as long as they do exist, the demand for the portfolio of assets mentioned will be very large, exceeding supply, which will drive the price of the portfolio up until arbitrage is no longer possible.

This chapter ${ }^{1}$ shows, by means of an example, that the mentioned belief can be incorrect when preferences change over time. Indeed, when preferences change over time it is possible that arbitrage opportunities exist even if the market is in equilibrium. Since Strotz's (1956) discussion of changing preferences, there has been an increasing interest in economic models thereof, including Akerlof (2002), Angeletos et al. (2001), Barro (1999), Frederick, Loewenstein, and O'Donoghue (2002), Gul and Pesendorfer (2001), Harris and Laibson (2001), Herings and Rohde (2006), Krusell and Smith (2003), Krusell, Kuruşçu, and Smith (2002), Laibson (1997), Loewenstein and Prelec (1992), Luttmer and Mariotti (2003), O'Donoghue and Rabin (1999), Phelps and Pollak (1968), Rubinstein (2003), and Thaler and Benartzi (2004).

When preferences change over time, several types of behavior can be distinguished. Naïve individuals erroneously believe that their preferences will not change over time (Pollak, 1968). Sophisticated individuals who do recognize that their preferences may change, either pre-commit themselves or only make plans that they expect to adhere to in the future. These individuals first try to predict their future behavior for every given possible current behavior. They may deliberately choose a suboptimal current behavior in order to force themselves not to

\footnotetext{
${ }^{1}$ This chapter is based on Rohde (2006).
} 
choose behavior in the future that is even worse from today's perspective. Akerlof (1991), Benhabib and Bisin (2005), DellaVigna and Malmendier (2004), Schelling (1984), Thaler and Shefrin (1981) give evidence and many examples thereof. In predicting their future behavior, individuals may predict their future preferences either correctly (fully sophisticated) or incorrectly (partly sophisticated; see Pollak, 1968, and Loewenstein, O’Donoghue, and Rabin, 2003).

Consider, as an illustration of the concept of sophistication, a smoker for whom it is optimal to smoke one cigarette per day. Suppose that he would indeed initially restrict himself to smoking only one cigarette a day. Then, after smoking that one cigarette, assume that he will be tempted even stronger to smoke a second cigarette and that he might indeed give in to that temptation, ending up smoking more than one cigarette per day. If this smoker would be naïve, he would not foresee this increased temptation after smoking the first cigarette, and would indeed smoke the one cigarette, ending up smoking more than one cigarette after all. If he would be sophisticated, he would foresee this temptation, and thus, know that he would never stick to only one cigarette per day. Knowing that his choice is restricted to either not smoking at all or smoking more than one cigarette, he may choose not to smoke at all.

This chapter gives an example of a market where an arbitrage opportunity exists in equilibrium, only because individual investors have changing preferences and want to influence future behavior. In the example, which is borrowed from Liebhafsky (1969), there is a possibility to buy an asset at zero costs today, which will yield a positive income tomorrow. Tomorrow, two goods can be consumed. More of these goods is always preferred. One of the goods is inferior from the perspective of tomorrow. From the perspective of today, however, that good is not inferior. Think of 'today' being the first half of an individual's life and 'tomorrow' being the second half. The normal good is a basket of goods corresponding to a luxurious life style and the upper social class of society. The inferior good is a less luxurious basket of goods corresponding to the middle class of society. Suppose that the individual expects that as his income rises he will tend to purchase more of the luxurious basket and less of the less luxurious one. From the perspective of the first half of his life he wishes he would keep buying the less luxurious basket corresponding to the middle class of society and not switch to the upper class in the future. One way to make sure that he stays in the middle class is to refrain his income from rising a lot.

\section{Illustration}

Assume that there are two periods: 'today' and 'tomorrow'. Today investors 
buy portfolios of assets that will pay off tomorrow. Tomorrow investors consume goods. There are two goods that can be consumed tomorrow: good 1 and good 2 . The expenditures are limited by income, which is determined by two components. The investor has an initial endowment of every good tomorrow. The value of endowments tomorrow and the payoffs tomorrow of the assets bought today together constitute income tomorrow. There are no market frictions, i.e. investors are allowed to go short or long in assets without bounds and tomorrow there are complete spot markets.

Consider an investor with endowments $\omega=\left(\omega_{1}, \omega_{2}\right)=(1 / 2,3 / 2)$, i.e. $1 / 2$ units of good 1 and $3 / 2$ units of good 2 . The prices of these two goods are expected to be equal to 1 tomorrow, and will indeed be equal to 1 tomorrow, i.e. consumers have rational price expectations (Radner, 1972). There is one asset that can be bought today at zero costs and that pays off 1 unit of money tomorrow. It will be shown later that such an asset can indeed exist when the market is in equilibrium. Thus, the income of the investor tomorrow will be equal to

$$
I=\omega_{1}+\omega_{2}+\theta
$$

where $\theta$ is the amount of assets bought today.

Today the preferences of the investor over consumption tomorrow are represented by the following utility function:

$$
U^{0}\left(x_{1}, x_{2}\right)=\min \left\{3 x_{1}, x_{2}\right\},
$$

where $x_{1}$ and $x_{2}$ are the consumption of good 1 and good 2 , respectively. The investor believes that his preferences over consumption will be different tomorrow, represented by

$$
U^{1}\left(x_{1}, x_{2}\right)=\frac{3}{4} \ln x_{1}+\frac{x_{2}^{2}}{2} .
$$

Thus, both today and tomorrow his utility is increasing in consumption, which means that he likes the goods.

It can be shown that with the latter preferences consumption tomorrow will depend on income $I$ as follows: either

$$
\begin{aligned}
& x_{1}^{*}=\frac{1}{2}\left(I-\sqrt{I^{2}-3}\right) \text { and } \\
& x_{2}^{*}=\frac{1}{2}\left(I+\sqrt{I^{2}-3}\right)
\end{aligned}
$$

or $x_{1}^{*}=I$ and $x_{2}^{*}=0$. Today the sophisticated investor believes that his consumption will be like this tomorrow and that his choice is restricted by (7.1) and (7.2). 
The only way in which he can influence tomorrow's consumption, is by influencing future income, through the sale or purchase of assets today.

Suppose that the investor decides not to invest in the costless asset, i.e. $\theta=0$. Then his income tomorrow will be equal to $I=\omega_{1}+\omega_{2}=1 / 2+3 / 2=2$, so that $x_{1}^{*}=1 / 2$ and $x_{2}^{*}=3 / 2$. Thus, he would keep his endowments.

Consider what would happen if he would decide to buy the asset. The curve in Fig. 7.1 shows tomorrow's demanded consumption bundles for various income levels. The dashed curve is an indifference curve from the perspective of today, that goes through $\omega$. From the perspective of today only bundles to the north-east of $\omega$ would be weakly preferred by the investor. However, by changing income, no bundles to the north-east of $\omega$ can be reached, given demand tomorrow. Thus, the investor will not buy the asset.

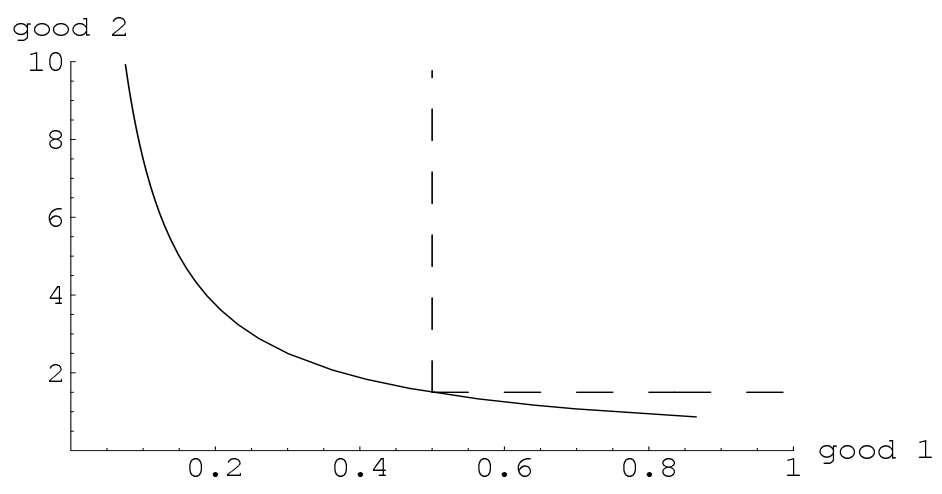

Figure 7.1: Tomorrow's consumption

Note that the utility functions of the investor are increasing in the consumption of the goods. Thus, tomorrow he would always wish he had more income. Also, a perfect pre-commitment device would allow the investor to specify today how he would like to spend his income tomorrow and would not allow him to choose to spend his income differently tomorrow. Then he would buy an infinite amount of the asset.

The figure shows that there can be investors who do want to either increase or decrease their future income by a limited amount. Consider a second investor who has the same preferences as the first investor, but less income from endowments in the second period. This investor wants to increase his income in the second period, until he can afford the consumption bundle $\omega$. Similarly, a third investor who has a higher income from endowments than our first investor, wants to decrease his 
income, until he will choose the consumption bundle $\omega$ tomorrow. Thus, the second investor will buy the asset, whereas the third investor will sell the asset. The endowments of the second and third investor can be chosen in such a way that demand and supply of the goods and the asset are exactly equal when the price of the asset is equal to zero. Thus, the second and third investor will trade the asset. This shows that in equilibrium there can indeed exist an asset in the market that (1) costs nothing, (2) yields a positive payoff in a future period, and (3) is traded.

This example shows that the existence of arbitrage opportunities in markets that are in equilibrium may not only be due to market frictions like, for instance, transaction costs, asymmetric information and restrictions to borrow money, but also by time-inconsistency of consumers' preferences. 



\section{Nederlandse Samenvatting (Summary in Dutch)}

\section{Intertemporele Keuzes en Competitief Evenwicht}

De meeste beslissingen die men neemt hebben direct of indirect te maken met intertemporele afwegingen. Door als kind naar school te gaan kunnen we op dat moment geen geld verdienen, maar investeren we in capaciteiten die het mogelijk maken later meer geld te verdienen. Veel mensen sparen of beleggen in aandelen, waardoor ze vandaag minder kunnen besteden in ruil voor meer mogelijkheden om geld uit te geven in de toekomst. Op een soortgelijke manier gaan kwesties omtrent het milieu meestal over de afweging tussen voordelige consumptie in het heden en een goed milieu in de toekomst.

Door de huidige vergrijzing van de bevolking, is er steeds meer aandacht voor ons pensioensysteem. De kosten voor de samenleving die veroorzaakt worden door de vergrijzing zijn enorm (Feldstein, 2005). Voor overheden is het steeds belangrijker om een goed systeem van pensioenen en sociale zekerheid te bieden. Het is daarom belangrijk om de intertemporele afwegingen die een samenleving wil maken goed te begrijpen. Dit proefschrift bestudeert zulke intertemporele afwegingen.

In economische en psychologische modellen wordt gewoonlijk aangenomen dat individuen huidige kosten en opbrengsten sterker wegen dan toekomstige. We zeggen dat individuen toekomstige kosten en opbrengsten verdisconteren. Sinds Samuelson's (1937) introductie van constant verdisconteerde utiliteit is dat model een gebruikelijke aanname geweest in economische analyses. Constant verdisconteren, ook wel bekend als exponentieel verdisconteren, impliceert dat voorkeuren over intertemporele afwegingen niet veranderen over tijd als de extra aanname wordt gemaakt dat voorkeuren over kosten en opbrengsten binnen periodes niet 
veranderen. Onder constant verdisconteren zullen we een vandaag genomen beslissing niet willen herzien in de toekomst, zolang er geen onvoorziene gebeurtenissen plaatsvinden. Constant verdisconteren impliceert dan dus tijdsconsistente voorkeuren.

Strotz (1956) was de eerste die een economisch model met tijdsinconsistente voorkeuren bestudeerde. Sinds de publicatie van zijn artikel zijn er veel psychologische en economische studies geweest die vonden dat individuen niet constant verdisconteren (Frederick, Loewenstein en O'Donoghue, 2002). Hyperbolisch verdisconteren bleek accurater te zijn in het beschrijven van intertemporele afwegingen die individuen maken. Als individuen niet constant, maar bijvoorbeeld hyperbolisch verdisconteren, veranderen hun voorkeuren meestal met de tijd. Als we willen dat economische modellen de realiteit representeren, dan vragen de vele schendingen van constant verdisconteren dus om een verwerping van de aanname dat voorkeuren tijdsconsistent zijn in die modellen. Tegenwoordig wordt veel onderzoek gedaan op dit gebied. De introductie van hyperbolisch verdisconteren in economische modellen heeft economen in staat gesteld fenomenen uit te leggen die voorheen niet verklaard konden worden, zoals, bijvoorbeeld, de sterke daling in consumptie rond pensionering en het feit dat consumenten vaak van zichzelf vinden dat zij te weinig sparen.

De introductie van tijdsinconsistente voorkeuren in economische modellen betekent niet alleen dat we de analyses van voorheen opnieuw moeten uitvoeren. Het vraagt ook om een herziening van de concepten waaruit economische modellen bestaan. Het is bijvoorbeeld niet direct duidelijk hoe traditionele welvaartsconcepten uitgebreid moeten worden in het geval van tijdsinconsistente voorkeuren. Welvaartsconcepten zijn essentieel bij het ontwerpen en beoordelen van overheidsbeleid en economische systemen. Tot op heden is er weinig onderzoek gedaan naar het definiëren van goede welvaartscriteria als voorkeuren veranderen met de tijd. In het tweede gedeelte van dit proefschrift worden zulke welvaartscriteria geïntroduceerd en worden economische systemen geanalyseerd als voorkeuren veranderen. Het ontwikkelen van nieuwe welvaartscriteria en de verdere analyse van economische systemen onder de aanname dat voorkeuren veranderen met de tijd, moet overheden en beleidsmakers uiteindelijk in staat stellen om, bijvoorbeeld, goede pensioensystemen te ontwerpen die de samenleving in staat stellen zo goed mogelijk met de vergrijzing om te gaan.

Het eerste deel van dit proefschrift bestudeert intertemporele voorkeuren op individueel niveau. Het tweede gedeelte bestudeert dynamische algemene evenwichtsmodellen en de bijbehorende welvaartscriteria. De volgende drie paragrafen geven een overzicht van het eerste gedeelte, het verband tussen de twee gedeeltes, 
en een overzicht van het tweede gedeelte.

\section{Intertemporele Keuzes}

Het eerste deel van dit proefschrift bestudeert intertemporele voorkeuren van individuen. Door dit hele deel heen nemen we verdisconteerde utiliteit aan. Onder verdisconteerde utiliteit waarderen individuen een stroom van uitkomsten als volgt. Ze berekenen eerst de utiliteit van elke uitkomst als die uitkomst meteen zou worden ontvangen. Vervolgens vermenigvuldigen ze elke utiliteit met de verdisconteringsfactor behorende bij het tijdstip van ontvangst van de uitkomst. Uiteindelijk tellen ze al deze verdisconteerde utiliteiten bij elkaar op. De som wordt dan de verdisconteerde utiliteit genoemd. Onder verdisconteerde utiliteit kan men tijdstippen waarop uitkomsten niet van de beslissing afhangen dus negeren bij het nemen van een beslissing.

Verdisconteerde utiliteit is een gebruikelijke aanname in de economie. Veel onderzoek in de economie en de psychologie richt zich op het type verdisconteringsfunctie. Samuelson (1937) bestudeerde constante verdiscontering, ook wel exponentiële verdiscontering genoemd. Bij constante verdiscontering verandert een voorkeur tussen twee stromen van uitkomsten niet als de ontvangst van alle uitkomsten in beide stromen wordt vertraagd met dezelfde tijdsperiode. Individuen die constant verdisconteren hebben een constant geduld. Vanaf vandaag gezien is hun geduld tussen vandaag en morgen niet anders dan hun geduld tussen 4 maart 2030 en 5 maart 2030.

Veel empirisch onderzoek heeft uitgewezen dat mensen en dieren niet constant verdisconteren (Frederick, Loewenstein en O'Donoghue, 2002). Veel studies vinden dat individuen minder ongeduldig zijn voor de verre toekomst dan voor de nabije toekomst, d.w.z. individuen zijn steeds geduldiger. (Quasi-) hyperbolisch verdisconteren kan dit stijgende geduld modelleren. Tegenwoordig is quasi-hyperbolisch verdisconteren steeds populairder aan het worden in toepassingen. Hyperbolisch en quasi-hyperbolisch verdisconteren zijn slechts twee alternatieven voor constant verdisconteren. Andere alternatieven zijn mogelijk, maar zijn nog niet onderzocht in de literatuur. De belangrijkste taken in het vakgebied van intertemporele keuzes zijn het beter leren begrijpen van de afwijking van constant verdisconteren en het ontwikkelen van goede alternatieven. Het eerste gedeelte van dit proefschrift draagt bij aan die taak.

Om een goed begrip te krijgen van de afwijking van constant verdisconteren, is het belangrijk om eerst te begrijpen wat constant verdisconteren precies is. Koopmans $(1960,1972)$ gaf een keuze-fundering van constant verdisconteren die erg populair is. Een keuze-fundering bestaat uit een aantal condities dat een 
keuzemodel volledig kenmerkt en dat gemakkelijk te interpreteren en empirisch te verifiëren is. Een keuze-fundering van constant verdisconteren geeft ons dus een goed inzicht in wat constant verdisconteren empirisch precies betekent. Helaas bevat Koopmans' analyse een aantal onnauwkeurigheden. Koopmans staat ook geen oneindige economische groei toe, wat onnodig beperkend is. Hoofdstuk 2 lost deze problemen op en geeft de eerste correcte uiteenzetting van Koopmans' klassieke resultaat.

Hoofdstuk 3 introduceert een maat voor de afwijking van constant verdisconteren. Die maat, de hyperbolische factor, kwantificeert de graad van stijgend geduld en de neiging tot tijdsinconsistent gedrag. De hyperbolische factor is gemakkelijk meetbaar. Hij geeft dus een gemakkelijk middel om (groepen van) mensen te identificeren die neigen tot tijdsinconsistent gedrag. Bovendien geeft de hyperbolische factor een makkelijke test voor hyperbolisch verdisconteren: de hyperbolische factor is constant dan en slechts dan als algemeen hyperbolisch verdisconteren geldt.

Hoofdstuk 4 introduceert zogenaamde tijdsafwegingsreeksen (TTO-reeksen) om de verdisconteringsfunctie en de mate van dalend of stijgend geduld te meten. Deze reeksen zijn heel eenvoudig te verkrijgen van individuen en kunnen meteen geanalyseerd worden met behulp van slechts pen en papier. Van TTO-reeksen kunnen de mate van stijgend geduld en de neiging tot tijdsinconsistent gedrag direct worden afgeleid uit eenvoudige grafieken. Door de constructie van TTOreeksen zijn er gedurende de hele procedure geen aannames over of metingen van utiliteit nodig. Bij vrijwel alle metingen van verdiscontering in de literatuur was het wel nodig om aannames over utiliteit te maken. Van TTO-reeksen kunnen hyperbolische factoren direct worden verkregen. We introduceren ook nog andere, heuristische maten van stijgend geduld.

In een experiment worden TTO-reeksen verkregen van subjecten. De resultaten laten zien dat de subjecten inderdaad niet voldoen aan constant verdisconteren. In tegenstelling tot de meeste studies in de literatuur, maar in overeenstemming met een aantal andere recente studies, vinden we bewijs voor dalend geduld. De hyperbolische factoren weerleggen ook de hyperbolische verdisconteringsfuncties die zo populair zijn in de literatuur. Deze bevindingen suggereren een aantal nieuwe richtingen voor toekomstig onderzoek in intertemporele keuzes.

\section{Intertemporeel Gedrag}

Zoals al eerder uitgelegd en aangetoond in het eerste deel van dit proefschrift, is er significant empirisch bewijs dat intertemporele voorkeuren van individuen niet aan constante verdiscontering voldoen. Als verdisconteerde utiliteit geldt en 
als voorkeuren over uitkomsten niet veranderen met de tijd, dan betekent een schending van constante verdiscontering dat individuen tijdsinconsistente, of dynamisch inconsistente, voorkeuren hebben. Er is ook ander bewijs voor het feit dat individuen tijdsinconsistente voorkeuren hebben. Individuen met tijdsinconsistente voorkeuren willen vaak eerder genomen beslissingen herzien, zelfs als er niets onvoorziens is voorgevallen. Daarom zullen individuen die zich er bewust van zijn dat ze tijdsinconsistente voorkeuren hebben, zichzelf willen vastleggen voor de toekomst om toekomstige herzieningen van beslissingen te voorkomen. Er zijn inderdaad gevallen bekend waarin individuen zich op dure wijze vastleggen voor de toekomst (Schelling, 1984).

Een klassiek voorbeeld van iemand die zich vastlegt voor de toekomst is Odysseus uit de Odyssee van Homerus. Odysseus riskeerde schipbreuk doordat hij met zijn schip langs een eiland met Syrenen moest varen, die zo mooi konden zingen dat ieder die hen hoorde het niet kon weerstaan naar hen toe te varen. Odysseus, die dat risico voorzag, liet zijn matrozen was in hun oren stoppen en liet zichzelf door hen aan de mast vastbinden, zodat hij de zang van de Syrenen zou kunnen horen zonder schade aan te richten.

Voor individuen met tijdsinconsistente voorkeuren veranderen de intertemporele voorkeuren eigenlijk met de tijd. Individuen met veranderende voorkeuren kunnen zich op verschillende manier gedragen, afhankelijk van hun zelfkennis. Naïeve individuen realiseren zich niet dat hun voorkeuren zullen veranderen met de tijd en zullen dus telkens verrast worden door hun onvoorziene voorkeuren. Zij zullen hun eerdere keuzes willen herzien en nieuwe plannen willen maken (Pollak, 1968). Verfijnde individuen, zoals Odysseus, voorspellen hun toekomstige voorkeuren correct en willen zichzelf vastleggen voor de toekomst of willen alleen plannen maken waarvan ze weten dat ze die ook uit zullen voeren. Gedeeltelijk verfijnde individuen voorspellen dat hun voorkeuren zullen veranderen in de toekomst, maar zij doen dit incorrect. Zij gedragen zich dus als verfijnde individuen, maar zullen toch elke periode verrast worden door hun voorkeuren.

\section{Algemeen Evenwicht}

Het tweede gedeelte van dit proefschrift analyseert het gedrag van naïeve en (gedeeltelijk) verfijnde individuen op geaggregeerd niveau in algemene evenwichtsmodellen. Een algemeen evenwichtsmodel bestaat uit consumenten en producenten die producten (goederen en diensten) verhandelen. Producenten beschikken over een technologie die productiefactoren zoals grond, arbeid en kapitaal gebruikt om goederen en diensten te produceren. In een zogeheten private eigendom economie zijn de producenten of bedrijven in het bezit van de consumenten, 
die een gegeven aantal aandelen in de bedrijven bezitten. Consumenten hebben voorkeuren over consumptie. Hun doel is zoveel mogelijk in hun behoeften te voorzien door producten te consumeren. Prijzen bepalen de verhoudingen waarin producten verhandeld worden tussen consumenten en producenten. De initiële bezittingen van de consumenten (met name arbeid en kapitaal) bepalen hun inkomen en vermogen. Consumenten zullen meestal hun inkomen willen verhogen om zoveel mogelijk producten te kunnen kopen. Zij zullen producenten dus dwingen een winst te behalen die het inkomen van de consumenten maximaliseert. Consumptie, productie en prijzen vormen dan een evenwicht als er geen vraagoverschot en geen aanbodsoverschot is. Markten zijn volledig competitief als er zoveel producenten en consumenten zijn dat een enkele producent of consument prijzen niet kan beïnvloeden. Consumenten en producenten nemen prijzen dan als gegeven en het evenwicht wordt dan een competitief evenwicht genoemd.

Het bekendste welvaartscriterium dat gebruikt wordt in algemene evenwichtstheorie, is Pareto efficiëntie. Een verdeling van producten wordt Pareto efficiënt genoemd als geen enkele consument beter af kan worden gemaakt zonder een andere consument slechter af te maken.

Traditionele algemene evenwichtsmodellen nemen aan dat voorkeuren van individuen niet veranderen met de tijd. Er kan dan worden aangetoond dat een competitief evenwicht altijd bestaat onder tamelijk lichte aannames. Het Eerste Fundamentele Welvaartstheorema houdt in dat elk competitief evenwicht in volledige markten Pareto efficiënt is. Markten zijn volledig als individuen alle mogelijke toekomstige producten nu al kunnen verhandelen door contracten op te stellen. Het Tweede Fundamentele Welvaartstheorema laat zien dat elke Pareto efficiënte verdeling van producten bereikt kan worden als een competitief evenwicht in volledige markten door een passende herverdeling van beginvoorraden. Deze stellingen laten zien dat, afgezien van herverdelingen van beginvoorraden, overheidsingrijpen alleen welvaartsverhogend is in het geval van externaliteiten, marktmacht, asymmetrische informatie of beperkingen in het verhandelen van toekomstige producten.

Bij de afleiding van deze resultaten werd altijd aangenomen dat voorkeuren niet veranderen met de tijd. Als voorkeuren wel kunnen veranderen met de tijd is het niet duidelijk of deze resultaten nog wel gelden. Het zou best kunnen zijn dat wanneer voorkeuren veranderen met de tijd, de rol van de overheid actiever moet zijn, zelfs als markten volledig zijn. Het is dan trouwens niet eens meer duidelijk hoe een competitief evenwicht en efficiëntie gedefinieerd moeten worden. Bij efficiëntie komt bijvoorbeeld de vraag naar boven welke voorkeuren moeten worden beschouwd in de concepten, de huidige, de toekomstige, of beide. Het 
tweede gedeelte van dit proefschrift introduceert veranderende voorkeuren in algemene evenwichtsmodellen. Voorkeuren nemen hier een algemene vorm aan en hoeven niet aan verdisconteerde utiliteit te voldoen. Omdat het opzetten van het model erg complex is, bekijken we alleen ruileconomieën, d.w.z. economieën zonder productie.

Hoofdstuk 5 introduceert evenwichts- en welvaartsconcepten in algemene evenwichtsmodellen waar consumenten niet vandaag al kunnen handelen in toekomstige producten. Wij zijn de eersten die algemeen veranderende voorkeuren opnemen in een volledig algemeen evenwichtsmodel. We introduceren nieuwe evenwichtsconcepten, laten zien welke problemen opduiken bij het definiëren van welvaartscriteria en laten zien hoe die problemen verholpen kunnen worden. We bewijzen dat evenwichten bestaan en dat sommige efficiëntie-concepten vervuld zijn in evenwicht, en andere niet.

Hoofdstuk 6 analyseert de volledigheid van markten. Markten zijn volledig als alle mogelijke conditionele producten kunnen worden verhandeld. Een conditioneel product is een product dat men vandaag betaalt en dat geleverd wordt in een toekomstige periode, conditioneel op een bepaalde gebeurtenis, zoals bijvoorbeeld futures, verzekeringen, aandelen en obligaties. Markten zijn sequentieel volledig als in elke periode alle conditionele producten voor de huidige en de volgende periode kunnen worden verhandeld. De Eerste en Tweede Welvaartstheorema zijn geldig in volledige alsook in sequentieel volledige markten. Beide marktstructuren leidden altijd tot dezelfde verdelingen van producten in evenwicht. Als voorkeuren veranderen met de tijd is het niet langer zo dat de twee marktstructuren dezelfde verdelingen opleveren wanneer individuen niet volledig verfijnd zijn, hetgeen wordt aangetoond in Hoofdstuk 6. Zo gauw individuen niet volledig verfijnd zijn, doet de graad van volledigheid van markten er toe.

Hoofdstuk 7 laat aan de hand van een voorbeeld zien dat er, in evenwicht, activa kunnen bestaan die vandaag niets kosten en die een positief inkomen in de toekomst opleveren. Dat voorbeeld geeft een indruk van wat de consequenties zijn van het introduceren van veranderende voorkeuren in economische modellen. Standaard economische modellen gaan ervan uit dat alle individuen hun toekomstige inkomen zo veel mogelijk willen laten stijgen. Het voorbeeld laat zien dat verfijnde individuen hun toekomstige inkomen niet per se willen laten stijgen. Het voorbeeld beschrijft een verfijnd individu dat weet dat hij morgen zijn inkomen gaat besteden op een manier die hij vandaag niet wil. Daarom kan het voorkomen dat arbitrage-mogelijkheden niet door de individuen in de markt worden weggenomen. Dit voorbeeld laat dus zien hoe bepaalde economische principes die zelden in twijfel getrokken worden, afhangen van de sterke aanname van tijdsconsistent gedrag. 



\section{REFERENCES}

Abdellaoui, Mohammed (2000), "Parameter-free Elicitation of Utilities and Probability Weighting Functions," Management Science, 46, 1497-1512.

Ainslie, George, and Nick Haslam (1992), "Hyperbolic Discounting," in G. Loewenstein and J. Elster (eds.), Choice over Time, Russell Sage Foundation, New York, pp. 57-92.

Airoldi, Mara, Daniel Read, and Shane Frederick (2005), "Longitudinal Dynamic Inconsistency," Work in Progress.

Akerlof, George A. (1991), "Procrastination and Obedience," American Economic Review, 81, 1-19.

Akerlof, George A. (2002), "Behavioral Macroeconomics and Macroeconomic Behavior," American Economic Review, 92, 411-433.

Angeletos, George-Marios, David I. Laibson, Andrea Repetto, Jeremy Tobacman, and Stephen Weinberg (2001), "The Hyperbolic Consumption Model: Calibration, Simulation, and Empirical Evaluation," Journal of Economic Perspectives, 15, 47-68.

Arrow, Kenneth J. (1953), "Le Rôle des Valeurs Boursières pour la Répartition la Meilleure des Risques," in Econométrie, Colloques Internationaux du Centre National de la Recherche Scientifique, 40, pp. 41-47; English version: "The Role of Securities in the Optimal Allocation of Risk-Bearing," Review of Economic Studies, 31, 91-96.

Arrow, Kenneth J., and Gérard Debreu (1954), "Existence of an Equilibrium for a Competitive Economy," Econometrica, 22, 265-290.

Attema, Arthur E., Han Bleichrodt, Kirsten I.M. Rohde, and Peter P. Wakker (2006), "Time-Tradeoff Sequences for Quantifying and Visualising the Degree of Time Inconsistency, Using only Pencil and Paper," Working Paper. 
Barro, Robert J. (1999), "Ramsey meets Laibson in the Neoclassical Growth Model," Quarterly Journal of Economics, 114, 1125-1152.

Bénabou, Roland J.M., and Jean Tirole (2002), "Self-Confidence and Personal Motivation, Quarterly Journal of Economics, 117, 871-915.

Benhabib, Jess, and Alberto Bisin (2005), "Modeling Internal Commitment Mechanisms and Self-Control: a neuroeconomics approach to consumption-saving decisions," Games and Economic Behavior, 52, 460-492.

Benzion, Uri, Amnon Rapoport, and Joseph Yagil (1989), "Discount Rates Inferred from Decisions: An Experimental Study," Management Science, 35, 270-284.

Bergstrom, Theodore C. (1975), "Maximal Elements of Acyclic Relations on Compact Sets," Journal of Economic Theory, 10, 403-404.

Bleichrodt, Han, and Amiram Gafni (1996), "Time Preference, the Discounted Utility Model and Health," Journal of Health Economics, 15, 49-67.

Bleichrodt, Han, and Magnus Johannesson (2001), "Time Preference for Health: A Test of Stationarity versus Decreasing Timing Aversion," Journal of Mathematical Psychology, 45, 265-282.

Bleichrodt, Han, Kirsten I.M. Rohde, and Peter P. Wakker (2005), 'Koopmans' Constant Discounting: A Simplification and an Extension to Incorporate Economic Growth," Working Paper.

Bommier, Antoine (2005), "Life-Cycle Theory for Human Beings," Working Paper.

Burness, H. Stuart (1976), "Impatience and the Preference for Advancement in the Timing of Satisfactions," Journal of Economic Theory, 6, 495-507.

Cairns, John, and Marjon van der Pol (2000), "Valuing Future Private and Social Benefits: The Discounted Utility Model versus Hyperbolic Discounting Models," Journal of Economic Psychology, 21, 191-205.

Camerer, Colin F., and Robin M. Hogarth (1999), "The Effects of Financial Incentives in Experiments: A Review and Capital-Labor-Production Framework," Journal of Risk and Uncertainty, 19, 7-42.

Chapman, Gretchen B. (1996), "Temporal Discounting and Utility for Health and Money," Journal of Experimental Psychology: Learning, Memory, and Cognition, 22, 771-791.

Chattopadhyay, Subir, and Piero Gottardi (1999), "Stochastic OLG Models, Market Structure, and Optimality," Journal of Economic Theory, 89, 21-67.

Citanna, Alessandro, Atsushi Kajii, and Antonio Villanacci (1998), "Constrained Suboptimality in Incomplete Markets: a General Approach and Two Applications," Economic Theory, 11, 495-521.

Coller, Maribeth, Glenn W. Harrison, and E. Elisabet Rutström (2005), "Does Everyone have Quasi-Hyperbolic Preferences?," Working Paper. 
Dasgupta, Partha S., and Eric S. Maskin (2005), "Uncertainty and Hyperbolic Discounting," American Economic Review, 95, 1290-1299.

Debreu, Gérard (1959), Theory of Value, John Wiley, New York.

Debreu, Gérard (1960), "Topological Methods in Cardinal Utility Theory," in Kenneth J. Arrow, S. Karlin, and Patrick Suppes (1959, eds.), Mathematical Methods in the Social Sciences, Stanford University Press, Stanford, CA, pp. 16-26.

DeGroot, Morris H. (1970), Optimal Statistical Decisions, McGraw-Hill, New York.

DellaVigna, Stephano, and Ulrike Malmendier (2004), "Contract Design and SelfControl: Theory and Evidence," Quarterly Journal of Economics, 119, 353402.

Diamond, Peter A. (1965), "The Evaluation of Infinite Utility Streams," Econometrica, 33, 170-177.

Dolmas, Jim (1995), "Time-Additive Representations of Preferences when Consumption Grows without Bound," Economics Letters, 47, 317-325.

Donaldson, John B., and Larry Selden (1981), "Arrow-Debreu Preferences and the Reopening of Contingent Claims Markets," Economics Letters, 8, 209-216.

Drèze, Jacques H., and P. Jean-Jacques Herings (2003), "Sequentially Complete Markets Remain Incomplete," Working Paper.

Dutta, Jayasri, and Stephen Morris (1997), "The Revelation of Information and Self-Fulfilling Beliefs," Journal of Economic Theory, 73, 231-244.

Feldstein, Martin (2005), "Rethinking Social Insurance," American Economic Review, 95, 1-24.

Fishburn, Peter C., and Ward Edwards (1997), "Discount-Neutral Utility Models for Denumerable Time Streams," Theory and Decision, 43, 139-166.

Fishburn, Peter C., and Ariel Rubinstein (1982), "Time Preference," International Economic Review, 23, 677-694.

Frederick, Shane (1999), "Discounting, Time Preference, and Identity," Ph.D. Thesis, Department of Social and Decision Sciences, Carnegie Mellon University.

Frederick, Shane, George F. Loewenstein, and Ted O'Donoghue (2002), "Time Discounting and Time Preference: A Critical Review," Journal of Economic Literature, 40, 351-401.

Geanakoplos, John D., and Herakles M. Polemarchakis (1986), "Existence, Regularity, and Constrained Suboptimality of Competitive Allocations when the Asset Market is Incomplete," in W.P. Heller, R.M. Starr, and D.A. Starrett (eds.), Uncertainty, Information and Communication: Essays in Honor of K.J. Arrow, Vol. III, Cambridge University Press, Cambridge, pp. 65-96. 
Gorman, William M. (1968), "The Structure of Utility Functions," Review of Economic Studies, 35, 367-390.

Green, Leonard, Nathanael Fristoe, and Joel Myerson (1994), "Temporal Discounting and Preference Reversals in Choice between Delayed Outcomes," Psychonomic Bulletin and Review, 1, 383-389.

Gruber, Jonathan, and Botond Kőszegi (2001), "Is Addiction 'Rational'? Theory and Evidence," Quarterly Journal of Economics, 116, 1261-1303.

Gul, Faruk, and Wolfgang Pesendorfer (2001), "Temptation and Self-control," Econometrica, 69, 1403-1435.

Haller, Hans H. (1990), "Non-Market Reopening, Time-Consistent Plans and the Structure of Intertemporal Preferences," Economics Letters, 32, 1-5.

Harris, Christopher, and David I. Laibson (2001), "Dynamic Choices of Hyperbolic Consumers," Econometrica, 69, 935-957.

Harvey, Charles M. (1986), "Value Functions for Infinite-Period Planning," Management Science, 32, 1123-1139.

Harvey, Charles M. (1995), "Proportional Discounting of Future Costs and Benefits," Mathematics of Operations Research, 20, 381-399.

Hellwig, Martin F. (1983), "A Note on the Implementation of Rational Expectations Equilibria," Economics Letters, 11, 1-8.

Herings, P. Jean-Jacques, and Herakles M. Polemarchakis (2005), "Pareto Improving Price Regulation when the Asset Market is Incomplete: an Example," Economic Theory, 25, 135-154.

Herings, P. Jean-Jacques, and Kirsten I.M. Rohde (2005), "On the Completeness of Complete Markets," Working Paper.

Herings, P. Jean-Jacques, and Kirsten I.M. Rohde (2006), "Time-Inconsistent Preferences in a General Equilibrium Model," Economic Theory, forthcoming.

Hertwig, Ralf, and Andreas Ortmann (2001), "Experimental Practices In Economics: A Challenge for Psychologists?," Behavioral and Brain Sciences, 24, 383-403.

Hübner, Ronald, and Reinhard Suck (1993), "Algebraic Representation of Additive Structure with an Infinite Number of Components," Journal of Mathematical Psychology, 37, 629-639.

Kirby, Kris N., and Nino N. Maraković (1995) "Modeling Myopic Decisions: Evidence for Hyperbolic Delay-Discounting within Subjects and Amounts," Organizational Behavior and Human Decision Processes, 64, 22-30.

Koopmans, Tjalling C. (1960), "Stationary Ordinal Utility and Impatience," Econometrica, 28, 287-309. 
Koopmans, Tjalling C. (1972), "Representations of Preference Orderings with Independent Components of Consumption," \& "Representations of Preference Orderings over Time," in C.B. McGuire and Roy Radner (eds.), Decision and Organization, North-Holland, Amsterdam, pp. 57-100,

Koopmans, Tjalling C., Peter A. Diamond, and Richard E. Williamson (1964), "Stationary Utility and Time Perspective," Econometrica, 32, 82-100.

Krantz, David H., R. Duncan Luce, Patrick Suppes, and Amos Tversky (1971), Foundations of Measurement, Vol. I (Additive and Polynomial Representations), Academic Press, New York.

Krusell, Per, and Anthony A. Smith (2003), "Consumption-Savings Decisions with Quasi-Geometric Discounting," Econometrica, 71, 365-375.

Krusell, Per, Burhanettin Kuruşçu, and Anthony A. Smith (2002), "Equilibrium Welfare and Government Policy with Quasi-Geometric Discounting," Journal of Economic Theory, 105, 42-72.

Laibson, David I. (1997), "Golden Eggs and Hyperbolic Discounting," Quarterly Journal of Economics, 112, 443-477.

Laibson, David I. (1998), "Life-Cycle Consumption and Hyperbolic Discount Functions," European Economic Review, 42, 861-871.

Liebhafsky, H.H. (1969), "New Thoughts about Inferior Goods," American Economic Review, 59, 931-934.

Loewenstein, George, and Drazen Prelec (1992), "Anomalies in Intertemporal Choice: Evidence and an Interpretation," Quarterly Journal of Economics, 107, 573-597.

Loewenstein, George, Ted O'Donoghue, and Matthew Rabin (2003), "Projection Bias in Predicting Future Utility," Quarterly Journal of Economics, 118, 1209 1248.

Luttmer, Erzo G.J., and Thomas Mariotti (2003), "Subjective Discounting in an Exchange Economy," Journal of Political Economy, 111, 1-30.

Luttmer, Erzo G.J., and Thomas Mariotti (2006), "Efficiency and Equilibrium when Preferences are Time-Inconsistent," Journal of Economic Theory, forthcoming.

Mas-Colell, Andreu, Michael D. Whinston, and Jerry R. Green (1995), "Microeconomic Theory," Oxford University Press, New York.

Mazur, James E. (1987), "An Adjusting Procedure for Studying Delayed Reinforcement," in Michael L. Commons, James E. Mazur, John A. Nevin, and Howard Rachlin (eds.), Quantitative Analyses of Behavior, Vol. 5: The Effect of Delay and of Intervening Events on Reinforcement Value, Hillsdale, NJ: Erlbaum, pp. 55-73. 
Mazur, James E. (2001), "Hyperbolic Value Addition and General Models of Animal Choice," Psychological Review, 108, 96-112.

Merton, Robert C. (1971), "Optimum Consumption and Portfolio Rules in a Continuous-Time Model," Journal of Economic Theory, 3, 373-413.

Meyer, Richard F. (1976), "Preferences over Time," in Ralph L. Keeney and Howard Raiffa (1976), Decisions with Multiple Objectives Wiley, New York (Second edition 1993, Cambridge University Press, Cambridge, UK), pp. 473 514.

O'Donoghue, Ted, and Matthew Rabin (1999), "Doing it Now or Later," American Economic Review, 89, 103-124.

Phelps, Edmund S., and Robert A. Pollak (1968), "On Second-Best National Saving and Game-Equilibrium Growth," Review of Economic Studies, 35, 185199.

Pollak, Robert A. (1968), "Consistent Planning," Review of Economic Studies, 35, 201-208.

Prelec, Drazen (2004), "Decreasing Impatience: A Criterion for Non-stationary Time Preference and 'Hyperbolic' Discounting," Scandinavian Journal of Economics, 106, 511-532.

Rachlin, Howard, and Andres Raineri (1992), "Irrationality, Impulsiveness, and Selfishness as Discount Reversal Effects," in G. Loewenstein and J. Elster (eds.), Choice over Time, Russell Sage Foundation, New York, pp. 93-118.

Radner, Roy (1972), "Existence of Equilibrium of Plans, Prices, and Price Expectations in a Sequence of Markets," Econometrica, 40, 289-303.

Read, Daniel, and N.L. Read (2004), "Time Discounting over the Lifespan," Organizational Behavior and Human Decision Processes, 94, 22-32.

Read, Daniel, Mara Airoldi, and Germán Loewe (2005), "Intertemporal Tradeoff Priced in Interest Rates and Amounts: A Study of Method Variance," Working Paper.

Read, Daniel, Shane Frederick, Burcu Orsel, and Juwaria Rahman (2005), "Four Score and Seven Years from now: The 'Date/Delay Effect' in Temporal Discounting," Management Science, forthcoming.

Rodriguez, Monica L., and A.W. Logue (1988), "Adjusting Delay to Reinforcement: Comparing Choice in Pigeons and Humans," Journal of Experimental Psychology: Animal Behavior Processes, 14, 105-117.

Rohde, Kirsten I.M. (2005), "The Hyperbolic Factor: a Measure of Decreasing Impatience," Working Paper.

Rohde, Kirsten I.M. (2006), "Arbitrage Opportunities in Frictionless Markets with Sophisticated Investors," Working Paper. 
Rubinstein, Ariel (2003), "'Economics and Psychology'? The Case of Hyperbolic Discounting," International Economic Review, 44, 1207-1216.

Samuelson, Paul A. (1937), "A Note on Measurement of Utility," The Review of Economic Studies, 4, 155-161.

Savage, Leonard J. (1954), The Foundations of Statistics, Wiley, New York. (Second edition 1972, Dover Publications, New York.)

Sayman, Serdar, and Ayse Oncüler (2005), "Reverse Time Inconsistency," Work in Progress.

Schelling, Thomas C. (1984), "Self-Command in Practice, in Policy, and in a Theory of Rational Choice," American Economic Review, 74, 1-11.

Streufert, Peter A. (1990), "Stationary Recursive Utility and Dynamic Programming under the Assumption of Biconvergence," Review of Economic Studies, $5 \%, 79-97$.

Streufert, Peter A. (1995), "A General Theory of Separability for Preferences Defined on a Countably Infinite Product Space," Journal of Mathematical Economics, 24, 407-434.

Strotz, Robert H. (1956), "Myopia and Inconsistency in Dynamic Utility Maximization," Review of Economic Studies, 23, 165-180.

Svensson, Lars E.O. (1976), "Sequences of Temporary Equilibria, Stationary Point Expectations and Pareto-Efficiency," Journal of Economic Theory, 13, 169183.

Thaler, Richard H. (1981), "Some Empirical Evidence of Dynamic Inconsistency," Economics Letters, 8, 201-207.

Thaler, Richard H., and Shlomo Benartzi (2004), "Save More Tomorrow: Using Behavioral Economics to Increase Employee Saving," Journal of Political Economy, 112, 164-187.

Thaler, Richard H., and Hersh M. Shefrin (1981), "An Economic Theory of SelfControl," Journal of Political Economy, 89, 392-406.

Wakker, Peter P. (1989), Additive Representations of Preferences, A New Foundation of Decision Analysis, Kluwer Academic Publishers, Dordrecht.

Wakker, Peter P. (1993a), "Unbounded Utility for Savage's 'Foundations of Statistics' and other Models," Mathematics of Operations Research, 18, 446-485.

Wakker, Peter P. (1993b), "Additive Representations on Rank-Ordered Sets II. The Topological Approach," Journal of Mathematical Economics, 22, 1-26.

Wakker, Peter P., and Daniel Deneffe (1996), "Eliciting von Neumann-Morgenstern Utilities when Probabilities Are Distorted or Unknown," Management Science, 42, 1131-1150. 
Wakker, Peter P., and Horst Zank (1999), "State Dependent Expected Utility for Savage's State Space; Or: Bayesian Statistics without Prior Probabilities," Mathematics of Operations Research, 24, 8-34. 


\section{AUthor IndeX}

Abdellaoui, Mohammed, 56

Ainslie, George, 70

Airoldi, Mara, 61

Akerlof, George, 28, 147, 148

Angeletos, George-Marios, 74, 147

Arrow, Kenneth, 80, 111, 112, 118, 133,134

Bénabou, Roland, 47

Barro, Robert, 147

Benartzi, Shlomo, 28, 47, 147

Benhabib, Jess, 70, 148

Benzion, Uri, 27

Bergstrom, Theodore, 139

Bisin, Alberto, 70, 148

Bleichrodt, Han, 18-20, 27

Bommier, Antoine, 61

Burness, Stuart, 22

Cairns, John, 27

Camerer, Colin, 62

Chapman, Gretchen, 41

Chattopadhyay, Subir, 112

Citanna, Alessandro, 85
Coller, Maribeth, 40

Dasgupta, Partha, 43, 61

Debreu, Gérard, 26, 98, 111, 118, 133, 134

DeGroot, Morris, 15, 16

DellaVigna, Stephano, 148

Deneffe, Daniel, 29

Diamond, Peter, 13, 17, 25

Dolmas, Jim, 16, 18, 19

Donaldson, John, 112

Drèze, Jacques, 112, 124

Dutta, Jayasri, 80

Edwards, Ward, 19, 20

Feldstein, Martin, 1

Fishburn, Peter, 13, 19, 20, 32, 35

Frederick, Shane, 1, 3, 12, 40, 61, 62, 70,147

Fristoe, Nathanael, 27, 63

Gafni, Amiram, 18-20

Geanakoplos, John, 85

Gorman, William, 18, 20, 22, 24, 26 
Gottardi, Piero, 112

Green, Jerry, 29

Green, Leonard, 27, 63

Gruber, Jonathan, 47

Gul, Faruk, 147

Hübner, Ronald, 16, 20

Haller, Hans, 112

Harris, Christopher, 28, 147

Harrison, Glenn, 40

Harvey, Charles, 13, 16-20, 28, 34, 43,51

Haslam, Nick, 70

Hellwig, Martin, 124

Herings, Jean-Jacques, 69, 85, 111, $112,124,147$

Hertwig, Ralf, 62

Hogarth, Robin, 62

Johannesson, Magnus, 27

Kajii, Atsushi, 85

Kirby, Kris, 28

Koopmans, Tjalling, 3, 11-22, 25, 26, 43

Krantz, David, 19, 20

Krusell, Per, 28, 71, 89, 147

Kuruşçu, Burhanettin, 71, 89, 147

Kőszegi, Botond, 47

Laibson, David, 12, 28, 35, 40, 47, 74, 147

Liebhafsky, H., 148

Loewe, Germán, 61

Loewenstein, George, 1, 3, 12, 28, 33, 34, 38, 40, 43, 51, 62, 70, $113,134,147,148$

Logue, A.W., 28
Luttmer, Erzo, 28, 71, 74, 85, 91, 112,147

Malmendier, Ulrike, 148

Maraković, Nino, 28

Mariotti, Thomas, 28, 71, 74, 85, 91, 112,147

Mas-Colell, Andreu, 29

Maskin, Eric, 43, 61

Mazur, James, 28, 34, 43, 51, 63

Merton, Robert, 12

Meyer, Richard, 18-20

Morris, Stephen, 80

Myerson, Joel, 28, 63

O'Donoghue, Ted, 1, 3, 12, 28, 40, 47, $62,70,113,134,147,148$

Oncüler, Ayse, 61

Orsel, Burcu, 61

Ortmann, Andreas, 62

Pesendorfer, Wolfgang, 147

Phelps, Edmund, 29, 35, 40, 43, 70, 147

Polemarchakis, Herakles, 85

Pollak, Robert, 5, 29, 35, 40, 43, 70, $74,112,119,134,147,148$

Prelec, Drazen, 28, 29, 31-34, 38, 40, $41,43,47,48,51,70,147$

Rabin, Matthew, 28, 47, 113, 134, 147, 148

Rachlin, Howard, 70

Radner, Roy, 80, 120, 149

Rahman, Juwaria, 61

Raineri, Andres, 70

Rapoport, Amnon, 27

Read, Daniel, 28, 61

Read, N.L., 28 
Repetto, Andrea, 74, 147

Rodriguez, Monica, 28

Rohde, Kirsten, 27, 51, 59, 63, 69, $111,112,147$

Rubinstein, Ariel, 20, 32, 35, 61, 147

Rutström, Elisabet, 40

Samuelson, Paul, 1, 3, 11, 27, 40

Savage, Leonard, 13

Sayman, Serdar, 61

Schelling, Thomas, 4, 148

Selden, Larry, 112

Shefrin, Hersh, 148

Smith, Anthony, 28, 71, 89, 147

Streufert, Peter, 16, 18-20, 24

Strotz, Robert, 1, 47, 147

Suck, Reinhard, 16, 19, 20

Svensson, Lars, 123

Thaler, Richard, 28, 43, 47, 70, 147, 148

Tirole, Jean, 47

Tobacman, Jeremy, 74, 147

van der Pol, Marjon, 27

Villanacci, Antonio, 85

Wakker, Peter, 13, 16-20, 24, 26, 29

Weinberg, Stephen, 74, 147

Whinston, Michael, 29

Williamson, Richard, 13, 25

Yagil, Joseph, 27

Zank, Horst, 24 



\section{SubJECT InDEX}

ageing, 1, 2

allocation, 73

time-consistent, 73

allocational equivalence, 124, 130

arbitrage, 7, 40, 44, 47, 123, 147, 148

choice anomaly, 3, 40, 47

commitment, 126, 130

compactified economy, 98

concavity

degree of, 48

Pratt-Arrow index of, 48

constant-equivalence, 16, 17

consumption bundle, 72

consumption plan, 72

consumption program, 13

consumption set, 72,75

expecations about, 72

contingent commodity, 6, 112

convexity

degree of, 48

decreasing impatience

Prelec's measure of, 41 decreasing-impatience index, see DIindex

demand set, 75, 80, 118, 121, 122, 128,129

DI-index, 49, 51, 57, 59, 60

discount factor, 14, 27, 33, 43

discount function, 28, 32, 42, 54

convexity index of, 40

measurement, 41, 52

discounted utility, 2, 3, 14, 17, 32, 39, $42,43,54,70$

violation of, 62

discounting, 1

constant, 1, 3, 4, 13, 17, 27, 29, $33,35,40,41,43,51,53,70$

exponential, see discounting, constant

general, 27, 42

generalized hyperbolic, 29, 33, 35, $40,43,51,52,59,63$

Harvey, 34, 35

hyperbolic, 1, 3, 4, 28, 40, 61, 70,81 
power, 53,61

proportional, 34, 43

quasi-hyperbolic, 3, 29, 35, 35, $40,43,51,57,63,70,81$ reversed, 61

dynamic consistency, 40

dynamic inconsistency, 28, 40

economy

locally non-satiated, 117

efficiency

CMP, see efficiency, constrained myopic periodical

constrained myopic periodical, 88, 89

constrained periodical, 93-95

$\mathrm{CP}$, see efficiency, constrained periodical

MOP, see efficiency, myopic overall Pareto

myopic overall Pareto, 84-86, 88

OP, see efficiency, overall Pareto overall Pareto, 91, 94

Pareto, 6, 85, 91, 92

TCOP, see efficiency, time-consistent overall Pareto

time-consistent overall Pareto, 9294

endowment, 5

equilibrium

AD, see equilibrium, Arrow-Debreu

Arrow-Debreu, 114, 115, 118, 124-

$126,130,131,133$

competitive, 5, 6, 76, 78

extended competitive, $76,77,78$, 81

naïve, $76,77,78,86-88,94,95$

existence of, 78 naïve complete, 114, 115, 128, 130-133

naïve SC, see equilibrium, naïve sequentially complete

naïve sequentially complete, 114 , 115, 121, 123, 125, 131-133

sophisticated, 80, 80, 81, 86, 87, 89, 93-95

existence of, 83

sophisticated complete, 114, 115, 129-131, 133

sophisticated SC, see equilibrium, sophisticated sequentially complete

sophisticated sequentially complete, 114, 115, 122, 123, 125, 126, 133

subgame perfect, 79, 113

event, 115

exchange economy, 6

expectations

correct, 120, 125, 128, 129

correct point, 80

point, 119

rational, 120, 125, 127

stationary point, 123

First Fundamental Theorem of Welfare Economics, 6

general equilibrium model, 5

Gini-index, 49

H, 31

decreasing impatience, 32

independence of outcomes, 33

independence of powers, 33

habit formation, 81

homogeneity, 99, 104 
household

naïve, 5, 71, 74, 112, 119, 147

partly sophisticated, 5, 119, 134, 148

sophisticated, 5, 71, 74, 112, 119, 147

hyperbolic factor, $3,4,27-29,31,31$, $32-35,51,57,59,63$

constant discounting, 33

generalized hyperbolic discounting, 33

Harvey discounting, 34

quasi-hyperbolic discounting, 35

impatience, 21, 30, 42

constant, 3, 30, 40, 43, 44, 47, 56-61, 63

decreasing, 3, 28, 30, 40-43, 43, $44,45,47-51,56-64$

measure of, 28, 29, 31, 32, 40

more than, 30, 41, 47, 48

increasing, 4, 30, 41, 42, 44, 45, 47-50, 56-64

more than, 48

incentive, 62

indifference pair, 31, 35

existence of, 31

irrationality, 28

markets

Arrow-Debreu, 113, 118

complete, 6, 113, 126, 127

perfectly competitive, 5

sequentially complete, $6,113,119$, 120

non-stationarity index, see NS-index

NS-index, 50, 51, 57, 59, 60
Odysseus, 4

opportunity set, $75,79,118,120-122$, 128

outcome, 30, 42

preferences over, 30

timed, 30

outcome stream, 42

simple, 46,53

pensionsystem, 1

planning period, 72

Pratt-Arrow index, 51

pre-commitment, 4

preference foundation, 3,11

preferences, 5,73

$\succeq^{* h, t}, 90$

acyclic, 126

complete, 14, 30, 75

consumption, over, 14

continuous, 30, 75

convex in present and future consumption, 75

dynamically inconsistent, 4

finite monotonicity, 21

impatient, see impatience

independence, 18

independence of past consumption, 81

initial-tradeoff independence, 14, 17

locally non-satiated in a state, 117

monotone, 14, 17, 21, 22, 75

monotonic, 30

open lower sections, 126

sensitive, 14, 17, 21

separability, 14, 18, 40

stationary, 14, 17, 18 
strictly convex in present and future consumption, 81

strong independence of past consumption, 90

time-inconsistent, 1, 4, 70, 74, 115,117

time-consistent, 1, 69, 73, 77, 78, $81,85,91,114,117,124-$ 126,131

transitive, 14, 30, 75

ultimately continuous, 15,17

weak order, 14, 17, 30

prices, 74

program

bounded, 17

constant, 14

consumption, see consumption program

ultimately constant, 15, 17, 21

unbounded, 15

ratio scale, 42

regularity, 32

scale compatibility, 62

Second Fundamental Theorem of Welfare Economics, 6

self, 70,79

separability, 14

social planner

forward-looking, 83

myopic, 83

society

naïve, 71

sophisticated, 71

spot markets, 119

state, 115,116

at period $t, 115$ stationarity, 30, 40-45, 47, 49-51, 56, 57,59

stopwatch time, 47

tail robustness, $17,21,22$

time

homogeneity, 47

time-inconsistency

stationarity, and, 43

time-tradeoff sequence, see TTO sequence

timed outcome, see outcome, timed preferences over, 30

timepoint, 30, 42

TTO curve, 45, 46, 48, 49, 57, 58, 60, 62

degree of concavity, 48 degree of convexity, 48

TTO sequence, 4, 40-42, 44, 44, 45, $51-57,62,63$

ultimate continuity, 15

uniqueness

up to unit and level, 17

utility, 14, 17, 40

bounded, 12, 25

unbounded, 13

utility function, 28, 32, 42, 75

Walras' law, 99, 104

welfare, 2

willingness to wait, $43,44,56,57,59$

WTW, see willingness to wait 




\section{Short Curriculum Vitae}

Kirsten Ingeborg Maria Rohde was born on March 4, 1980 in Eindhoven, the Netherlands. She attended a Dutch primary school in Madrid, Spain, from 1982 to 1985. From there she attended a primary school in France from 1985 to 1989. She graduated with distinction (cum Laude) from high school (VWO) in the Netherlands in 1998. She then proceeded to Maastricht University to study econometrics, specifically mathematical economics, from 1998 to 2002, during which she worked as a research assistant in finance. She obtained her master's degree with distinction in 2002 with a thesis that earned the Thesis Award of Maastricht University.

Kirsten was a PhD student at the Department of Economics of Maastricht University under the supervision of Professors Jean-Jacques Herings and Peter Wakker from 2002 to 2006. She was a visiting fellow at the Institute for Empirical Research in Economics at the University of Zürich in the spring of 2006.

Kirsten Rohde's research interests: decision theory, mathematical economics, experimental economics, psychology, game theory, microeconomic theory and general equilibrium theory. 
